\title{
DETERMINATION OF CONCENTRATION-DEPENDENT DISPERSION OF PROPANE IN VAPOR EXTRACTION OF HEAVY OIL
}

\author{
by \\ Hadil Abukhalifeh \\ B.Sc in Chemical Engineering, Kuwait University, Kuwait, 1986 \\ M.A.Sc in Chemical Engineering, Ryerson University, Toronto, 2004
}

A dissertation presented to

Ryerson University

\begin{abstract}
in partial fulfillment of the
requirements for the degree of

Doctor of Philosophy

in the program of

Chemical Engineering
\end{abstract}

Toronto, Ontario, Canada, 2010

CHadil Abukhalifeh 2010 


\section{Author's declaration}

I hereby declare that I am the sole author of this dissertation.

I authorize Ryerson University to lend this dissertation to other institutions or individuals for the purpose of scholarly research

$H$ adil $A$ bukhalifeh

I further authorize Ryerson University to reproduce this dissertation by photocopying or by other means, in total or in part, at the request of other institutions or individuals for the purpose of scholarly research.

$\mathrm{H}$ adil $\mathrm{A}$ bukhalifeh 


\title{
DETERMINATION OF CONCENTRATION-DEPENDENT DISPERSION OF PROPANE IN VAPOR EXTRACTION OF HEAVY OIL \\ Doctor of Philosophy \\ 2010 \\ Hadil Abukhalifeh \\ Chemical Engineering \\ Ryerson University
}

\begin{abstract}
Vapex (vapor extraction) is a solvent-based non-thermal in-situ heavy oil recovery process. In Vapex process, a vaporized hydrocarbon solvent is injected into an upper horizontal well where the solvent mixes with the heavy oil and reduces its viscosity. The diluted oil drains under gravity to a bottom production well. Two mechanisms control the production rates of heavy oil in Vapex: mass transfer of solvent into heavy oil, and gravity drainage. Both are governed by dispersion, which is composed of molecular diffusion, convection, and other mechanisms that enhance mixing in porous medium. The accurate determination of solvent dispersion in Vapex is essential to predict effectively the amount and time scale of oil recovery as well to optimize the field operations.

Motivated by limited dispersion data in the literature, a novel technique is developed to determine experimentally the concentration-dependent dispersion coefficient of propane in Vapex process. The technique employs live oil production rates obtained from Vapex experiments at $21^{\circ} \mathrm{C}$ and $0.790 \mathrm{MPa}$. The salient feature of this technique is that it does not impose any functional form on dispersion as a function of concentration, but allows its
\end{abstract}


natural and realistic determination. The technique could be applied to determine other solvents dispersion coefficient used in the in-situ recovery of heavy oil.

Propane dispersion coefficient is determined by the minimization of the difference in experimental and calculated cumulative live oil produced. The necessary conditions for the minimum are fundamentally derived, utilizing the theory of optimal control. A computational algorithm is formulated to calculate the propane dispersion function simultaneously with propane-heavy oil interface mass fraction. Physical models of glass beads of different permeabilities (204-51 Darcy) and drainage heights $(25-45 \mathrm{~cm})$ were used to conduct the Vapex experiments. The results show that dispersion of propane is a unimodal function of its concentration in heavy oil, and lies in the range, $0.5 \times 10^{-5}-7.993 \times 10^{-5} \mathrm{~m}^{2} / \mathrm{s}$. Convectional mixing is promoted by higher model drainage heights and lower permeability. Finally, propane dispersion is correlated as a function of propane mass fraction in heavy oil and the packed medium permeability, as well as the drainage height. 


\section{Acknowledgements}

I would like to thank my supervisors and teachers Dr. Ali Lohi and Dr. Simant Ranjan Upreti for their guidance, encouragement, and support throughout the course of this project. I learned a lot from their great personality as well as the scientific knowledge and experience.

I am very grateful to Mr. Ali Hemmati for all of his friendly and helpful technical supports during performing the experiments. The technical assistance of Mr. Daniel Boothe is also appreciated. Thanks are also extended to Mr. Tondar Tajrobehkar for his great technical help.

Financial support provided by NSERC, OGS, and Department of Chemical Engineering are greatly acknowledged. 


\section{Dedication}

To My Family 


\section{Table of Contents}

Author's declaration—_ii

Abstract—_iii

Acknowledgments $-\quad \mathrm{V}$

Dedication- $-v i$

Table of Contents—

List of Tables- $-\mathrm{xi}$

List of Figures—- xiii

List of Symbols and Nomenclature_—

Chapter 1: Introduction-

1.1 General Aspects about Heavy Oil-_ 2

1.2 Canada's Oil Sands__ 5

1.3 The Vapex Process__ 7

1.4 Dispersion—_ 9

1.5 Optimal Control Theory-_ 11

1.6 Research Objectives-_ 13

1.7 Structure of the Dissertation- 14

Chapter 2: Literature Review- 15

2.1 History of Vapex-15

2.2 Diffusion and Dispersion Phenomena- 16

2.2.1 Molecular Diffusion- 16

2.2.2 Concentration Dependence of Molecular Diffusion- 17

2.2.3 Dispersion in Porous Medium—20

2.3 Dispersion in Vapex- 22

2.4 Diffusion and Dispersion of Propane in Vapor Extraction of Heavy Oil —-24

2.5 Approach of this Work- 28 
3.1 Experimental Setup- 31

3.2 Sample Preparation- 35

3.3 Bitumen Properties—_ 35

3.4 Glass Beads Properties- 35

3.5 Experimental Procedures—_ 38

3.6 Live Oil Viscosity Measurement_— 39

3.7 Live Oil Density and Amount of Dissolved Propane Measurements__ 41

3.8 Experimental Error- -41

3.9 Residual Oil Saturation- 42

Chapter 4: Theory and Computations- 45

4.1 Mass Transfer Model of Vapex- -45

4.2 The Optimal Control Problem — 51

4.2.1 Objective Functional $\longrightarrow 51$

4.2.2 Necessary Conditions - 53

4.2.3 Adjoint Equations_— 59

4.3 Solution of the Optimal Problem- 60

4.3.1 The Gradient Improvement Method-_ 61

4.3.2 Implementation of the Improvement Method- -61

4.3.3 Integration of Continuity and Adjoint Equations-_ 62

4.3.4 Non-Dimensionalization of the Model- -63

4.3.5 The Optimal Control Algorithm- -73

4.3.6 Initialization of the Algorithm Variables-

Chapter 5: Results and Discussion

5.1 Experimental Results and Discussion $\longrightarrow 77$

5.1.1 System Pressure and Temperature- 78

5.1.2 Live Oil Properties_— 79

5.1.2.1 Propane Solubility — 79

5.1.2.2 Live Oil Density — 80 
5.1.2.3 Live Oil Viscosity — 81

5.1.3 Live Oil Production Rates__ 83

5.2 Numerical Results and discussion- -87

5.2.1 Algorithm Execution- -87

5.2.2 Propane-Heavy Oil Interface Mass Fraction-_ 89

5.2.3 Determination of Concentration-Dependent Dispersion Coefficient-91

5.2.4 Model Validation- -95

5.2.5 Calculated Change of Bitumen Height in the Porous Medium- 96

5.2.6 Sensitivity Analyses of the Model Parameters__ 96

5.3 Permeability Effect on Concentration-Dependent Dispersion Coefficient_—98

5.4 Drainage Height Effect on Concentration-Dependent Dispersion Coefficient-104

5.5 Permeability and Drainage Height Effect on Calculated Bitumen Height_— 114

5.6 Correlation of Dispersion Data- 116

5.7 Significance of the Dispersion Data_ 118

Chapter 6: Conclusions and Recommendations

6.1 Conclusions- - 121

6.2 Recommendations_—_ 123

References-

Appendix A: Porous Medium Permeability Calculation- 131

Appendix B: Live Oil Viscosity Calculations - 133

Appendix C: Live Oil Density and Amount of Propane Dissolved in Heavy Oil

Calculations 135

Appendix D: Recorded mass of the physical model with time- 137

Appendix E: Relative deviation of the pressure vessel and the water bath temperatures - 139

Appendix F: Cumulative Oil Production Curves for various physical models-_ 141

Appendix G: A Comparison between the Simulation Results for both 60, 180 Minutes - 145

Appendix H: Justification of extrapolation of the viscosity model -151

Appendix I: Velocity profile at the bottom of the physical model -153 


\section{List of Tables}

Table 2.1: Details of studies on the determination of propane dispersion/effective diffusivity in Vapex — 28

Table 3.1: Properties of the heavy oil- 35

Table 3.2: Specifications of the glass beads_-36

Table 3.3: Permeability of the glass beads_— 38

Table 3.4: Instrument's range and accuracy-_ 42

Table 5.1: Average propane mass fraction in produced oil for various physical models_— 80

Table 5.2: Average live oil density $\left(\mathrm{kg} / \mathrm{m}^{3}\right)$ for various physical models $— 81$

Table 5.3: Average live oil viscosity (cP) for various physical models_- 82

Table 5.4: Experimental live oil production rates $(\mathrm{g} / \mathrm{min})$ for various packing permeability and heights -85

Table 5.5: Calculated average drainage live oil velocity $(\mathrm{m} / \mathrm{s})$ for various packing $—-86$

Table 5.6: Simulation model parameters (204 D and $25 \mathrm{~cm}) \_89$

Table 5.7: Objective functions at various interface mass fractions of propane and dispersion values -90

Table 5.8: Change in Average dispersion values_— 97

Table 5.9: Maximum and average dispersion values and corresponding mass fraction for different drainage heights-_103

Table 5.10: Maximum and average dispersion and corresponding mass fraction for different packed medium permeabilities_- 110

Table 5.11: Regressed values of the parameters in Equations (5.2)__ 117

Table 5.12: Regressed values of the parameters in Equations (5.3)_-118 


\section{List of Figures}

Figure 1.1: World history and projected energy consumption (EIA)—_ 3

Figure 1.2: Historical and projected unconventional oil production (EIA)___ 4

Figure 1.3: countries with significant amounts of bitumen— 4

Figure 1.4: Canada's heavy oil and bitumen (Wikimedia 2006)—

Figure 1.5: Side view of Vapex- 7

Figure 1.6: Cross sectional view of Vapex- 8

Figure 1.7 Sketch showing diffusion, convection and dispersion in a capillary tube (John, 2008)— 10

Figure 2.1: Overall diffusion coefficient for toluene-bitumen system (after Oballa and Butler,1989)__ 18

Figure 2.2: Concentration dependence of diffusivity (after Mokrys and Butler, 1989)—19

Figure 2.3: Concentration dependence of diffusivity (after Luo et al., 2007)—-20

Figure 2.4: Schematic of methodology for this work- 30

Figure 3.1: Schematic diagram of the experimental setup_-32

Figure 3.2: Picture of Vapex Experimental Set_- 34

Figure 3.3: Particle size distribution- 36

Figure 3.4: Experimental setup for permeability measurement_—_ 37

Figure 3.5: Experimental Setup for Live Oil Viscosity Determination_— 40

Figure 3.6: Pressure drop across capillary tube versus time as recorded by data acquisition system- 40

Figure 3.7: Samples from different sections of the model

Figure 4.1: Differential element of the physical model_— 47

Figure 4.2: Cylindrical model and grid distribution- -63

Figure 4.3: The Optimal Control algorithm—_ 74

Figure 4.4: Solvent-heavy oil interface-_ 75

Figure 5.1: Temperature profile for the pressure vessel and the water bath

(204 Darcy and $35 \mathrm{~cm}$ )— 78

Figure 5.2: Vessel pressure versus time (35 cm, $204 \mathrm{D}) \longrightarrow 79$

Figure 5.3: Live oil viscosity versus propane mass fraction- -82 
Figure 5.4: Cumulative live oil production versus time $(25 \mathrm{~cm}) \longrightarrow 84$

Figure 5.5: Cumulative live oil production versus time (204 D)—_ 85

Figure 5.6: Objective functions versus solvent interface mass fraction for initial dispersion of $0.5 \times 10^{-5} \mathrm{~m}^{2} / \mathrm{s} \longrightarrow 91$

Figure 5.7: Objective function versus iteration number (204 D and $25 \mathrm{~cm}) \longrightarrow 92$

Figure 5.8: Dispersion coefficient of propane in heavy oil (204 D, $25 \mathrm{~cm}) \longrightarrow 93$

Figure 5.9: Experimental and calculated mass of live oil produced with time $(204 \mathrm{D}, 25 \mathrm{~cm}) \longrightarrow 94$

Figure 5.10: Model validation (204 D and $25 \mathrm{~cm}$ )— 95

Figure 5.11: The change in bitumen height with time (204 D, $25 \mathrm{~cm})-96$

Figure 5.12: Objective function versus number of iteration for $25 \mathrm{~cm}$ drainage height— -98

Figure 5.13: Dispersion coefficients of propane in heavy oil with three medium permeabilities (25 cm drainage height)__ 99

Figure 5.14: Dispersion coefficients of propane in heavy oil with three medium permeabilities (35 cm drainage height)—_ 100

Figure 5.15: Dispersion coefficients of propane in heavy oil with three medium permeabilities (45 cm drainage height) $-100$

Figure 5.16: Experimental and calculated cumulative mass of oil produced with time for three medium permeabilities (25 cm drainage height)__ 101

Figure 5.17: Experimental and calculated cumulative mass of oil produced with time for three medium permeabilities (35 cm drainage height)__ 102

Figure 5.18: Experimental and calculated cumulative mass of oil produced with time for three medium permeabilities (45 cm drainage height)-_ 102

Figure 5.19: Objective function versus iteration number for $204 \mathrm{D}$ model -105

Figure 5.20: Experimental and calculated cumulative mass of oil produced with time for different model heights with 204 D permeability_-106

Figure 5.21: Experimental and calculated cumulative mass of oil produced with time for different model heights with $102 \mathrm{D}$ permeability 106

Figure 5.22: Experimental and calculated cumulative mass of oil produced with time for different model heights with $51 \mathrm{D}$ permeability 107 
Figure 5.23: Dispersion coefficients of propane in heavy oil for 204 D permeability with different model heights 108

Figure 5.24: Dispersion coefficients of propane in heavy oil for $102 \mathrm{D}$ permeability with different model heights__ 108

Figure 5.25: Dispersion coefficients of propane in heavy oil for $51 \mathrm{D}$ permeability with different model heights_- 109

Figure 5.26: Average live oil viscosity and velocity versus drainage height___ 111

Figure 5.27: Average dispersion coefficients versus drainage height with various permeabilities

Figure 5.28: Average dispersion versus experimental average propane mass fraction_—113

Figure 5.29: Propane dispersion versus corresponding propane mass fraction- -114

Figure 5.30: The change in bitumen height with time (204,102 and 51 D) and drainage height of $25 \mathrm{~cm}$ 115

Figure 5.31: The change in bitumen height with time $(25,35$ and $45 \mathrm{~cm})$ and packing permeability of $102 \mathrm{D}$ 116 


\title{
List of Symbols and Nomenclature
}

\author{
A $\quad$ area, $\mathrm{m}^{2}$ \\ $D(\omega) \quad$ dispersion coefficient of solvent in medium, $\mathrm{m}^{2} / \mathrm{s}$ \\ $D_{p} \quad$ diameter of the particle, $\mathrm{m}$ \\ $g \quad$ gravity, $\mathrm{m} / \mathrm{s}^{2}$ \\ I objective functional defined by Equation (4.14) \\ $J \quad$ augmented objective functional defined by Equation (4.21) \\ $J_{\mathrm{f}} \quad$ dispersive flux of solvent in the medium along the radial direction, $\mathrm{kg} / \mathrm{m}^{2} \cdot \mathrm{s}$ \\ $\mathrm{K}$ permeability of the medium, D \\ $\mathrm{K}_{\mathrm{r}} \quad$ relative permeability of the medium \\ $m_{c} \quad$ calculated mass of the produced live oil, $\mathrm{kg}$ \\ $m_{e} \quad$ experimental mass of the produced live oil, $\mathrm{kg}$ \\ $\mathrm{P} \quad$ Pressure, $\mathrm{MPa}$ \\ $r \quad$ distance along the radial direction, $\mathrm{m}$ \\ $R \quad$ radius of cylindrical medium, $\mathrm{m}$ \\ $S \quad$ surface area, $\mathrm{m}^{2}$ \\ T temperature, ${ }^{\circ} \mathrm{C}$ \\ $t \quad$ time, $\mathrm{s}$ \\ $u \quad$ interstitial velocity, $\mathrm{m} / \mathrm{s}$ \\ $V \quad$ volume of a finite element in the medium, $\mathrm{m}^{3}$ \\ $v \quad$ Darcy velocity, $\mathrm{m} / \mathrm{s}$ \\ $z \quad$ distance along the vertical direction, $\mathrm{m}$ \\ $Z \quad$ bitumen height in the medium at a given $r$ and $t, \mathrm{~m}$ \\ $Z_{0} \quad$ initial height, $\mathrm{m}$
}

Greek Symbols

$\varphi \quad$ porosity of the medium

$\gamma \quad$ adjoint variable defined by Equation (4.48) 
$\lambda \quad$ adjoint variable defined by Equation (4.47)

$\mu \quad$ viscosity of the live oil, $\mathrm{cP}$

$\mu_{0} \quad$ viscosity coefficient of the live oil, $\mathrm{kg} / \mathrm{m} \cdot \mathrm{s}$

$\rho \quad$ density of the live oil, $\mathrm{kg} / \mathrm{m}^{3}$

$\omega \quad$ mass fraction of solvent in bitumen

$\omega_{\text {int }} \quad$ mass fraction of solvent at the solvent-heavy oil interface

$\alpha \quad$ dispersivity of the porous medium, $\mathrm{m}$

Abbreviations

BFGS Broyden Fletcher Goldfarb and Shanno Method

CMG Computer Modeling Group LTD.

D Darcy

EOR Enhanced Oil Recovery

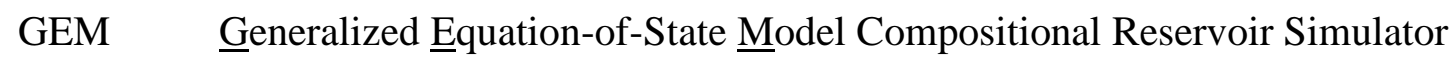

PV Pore Volume

Vapex Vapor extraction 


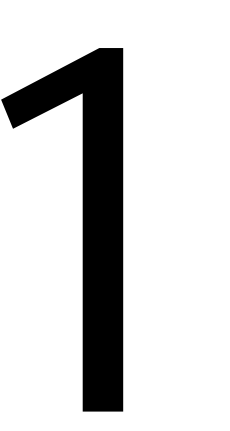

\section{Introduction}

Western Canada has approximately 175 billion barrels of heavy oil in place. The challenge is to develop energy efficient and environmentally acceptable recovery techniques to recover heavy oil and bitumen. Heavy oil is a type of crude oil that is very viscous and does not flow easily. Traditional methods of oil production and extraction are not applicable in oil reservoirs with heavy oil or bitumen due to its high viscosity. Steam-based methods are often employed to recover heavy oil by reducing the viscosity of in-situ heavy oil. Another alternative is the solvent-based methods, which require no water and the solvent consumed is recoverable and usable. Vapex (vapor extraction) is a promising solvent-based method to produce heavy oil and bitumen by reducing the heavy oil viscosity through the injection of a light hydrocarbon solvent into the reservoir.

This thesis aims to determine the concentration-dependent dispersion coefficient of propane in heavy oil in Vapex process. This goal is achieved by applying optimal control theory to determine the solvent dispersion function that minimizes the discrepancy between the experimental and model-calculated oil production rates. Vapex experiments are carried out using a physical model of heavy oil saturated with glass beads in a cylindrical geometry. An optimal control technique is developed to determine the solvent dispersion as a function of its concentration in heavy oil. In addition, the effects of the packed medium permeability and drainage height on propane dispersion coefficient are investigated. 
The dispersion of propane determined as a function of its concentration in heavy oil will enable more accurate reservoir simulations and help to optimize Vapex operation conditions.

\subsection{General Aspects about Heavy Oil}

Energy is a vital component for growth and sustainable development. The primary energy source in the world is petroleum. Its reserves can be classified into conventional (crude oil) and nonconventional (heavy oil and bitumen) based on their viscosities and API gravities. Heavy oil and bitumen, often called tar sands or oil sands, differ from light oils by their high viscosity at reservoir temperatures, low API gravity, and significant amounts of nitrogen, oxygen, sulphur and heavy-metal contents (vanadium, nickel, iron). The heavy oil has a viscosity greater than $1000 \mathrm{cP}$ (water is $1 \mathrm{cP}$ ) with API gravity of $20^{\circ}$ or less, while bitumen viscosity is greater than $10,000 \mathrm{cP}$ with API gravity of $10^{\circ}$ or less (Speight, 2009). The heavy oil and bitumen resemble the residuum from the refining of light oil. Most heavy oil and bitumen are found at the margins of geologic basins and are thought to be the residue of formerly light oil that has lost its lightmolecular-weight components through degradation by bacteria, water washing, and evaporation.

Figure 1.1 shows the total energy consumed historically from 1990 to 2006 and total projected energy consumption from 2010 to 2030. The total world consumption of energy is projected to increase by 44 percent from 2006 to 2030 . The projected forecasts indicate that liquid fuel consumption will continue to increase annually. As the growth in liquid fuel demand continues, investments are attracted to heavy oil and bitumen reserves to supplement conventional oil supplies. These reserves are much more costly to extract, transport, and refine than conventional oils, but depletion of the conventional resources has turned the world towards unconventional resources. 


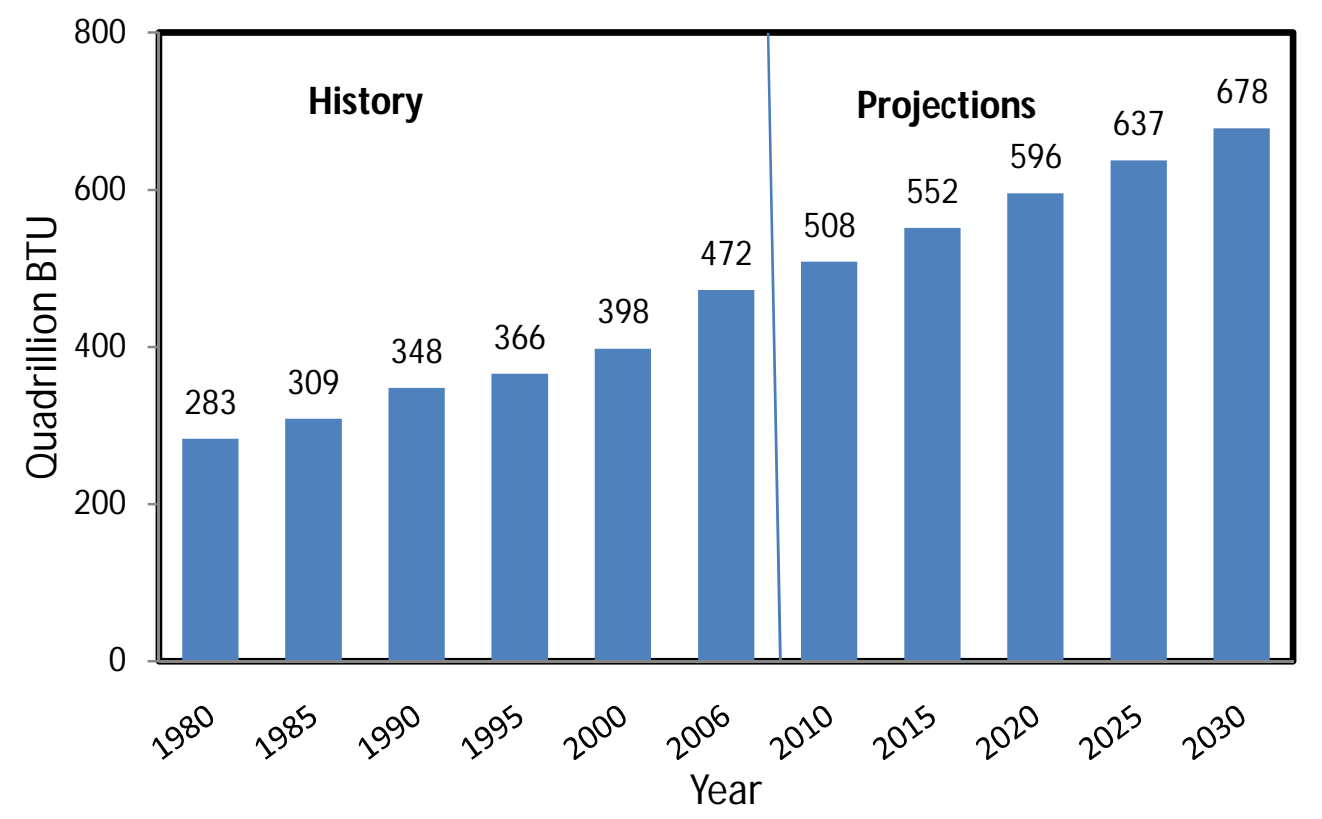

Figure 1.1 World history and projected energy consumption (EIA)

Unconventional resources, which include oil sands, extra-heavy oil, biofuels, coal-to-liquids, and gas-to-liquids, are expected to become increasingly competitive as shown in Figure 1.2. The figure shows that the total production of unconventional liquid fuel is expected to increase from 3.2 million barrels of oil per day in 2006 to more than 13 million barrels of oil per day in 2030 . Bitumen and heavy oil are expected to comprise of about $60 \%$ of the unconventional oil production in 2030. 


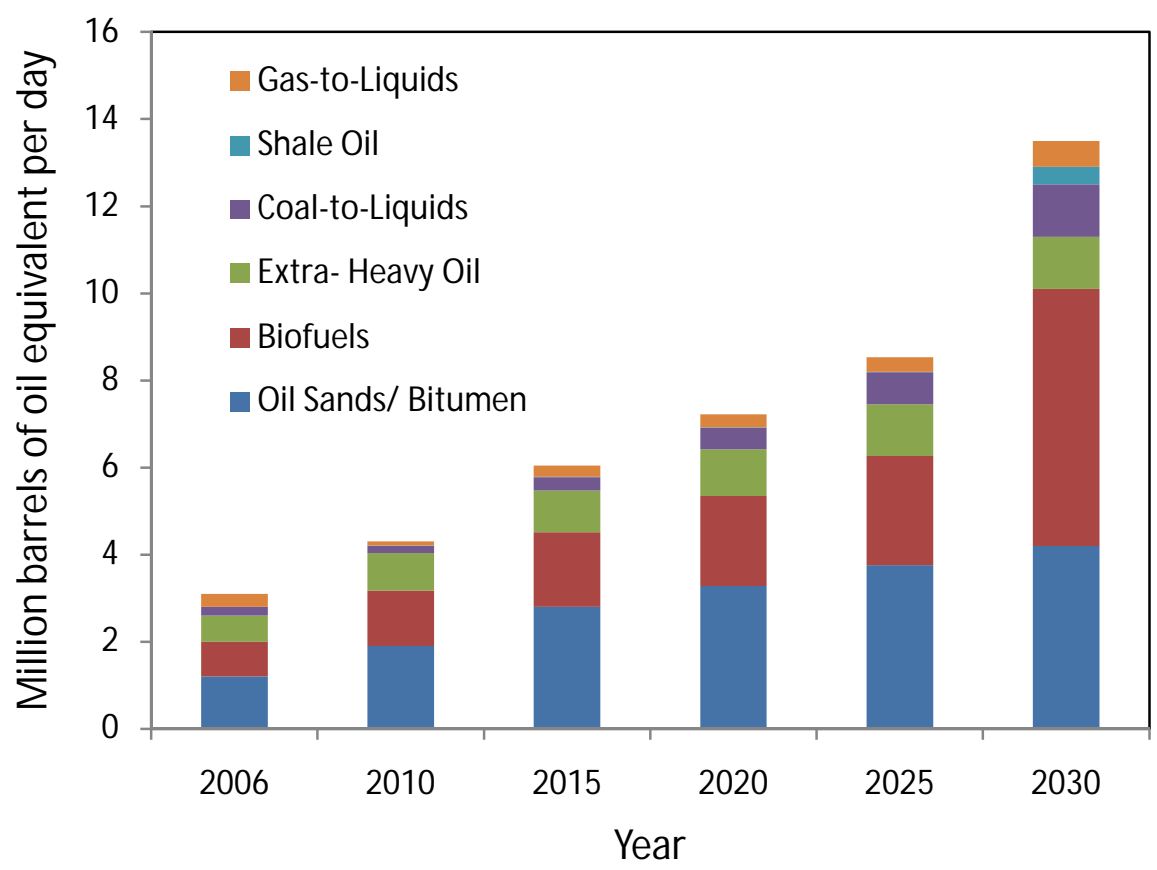

Figure 1.2 Historical and projected unconventional oil production (EIA)

Figure 1.3 shows the countries with significant amounts of bitumen in place not proven recoverable oil. Canada, Venezuela and Russia have the highest amount of bitumen in place (Smalley 2000).

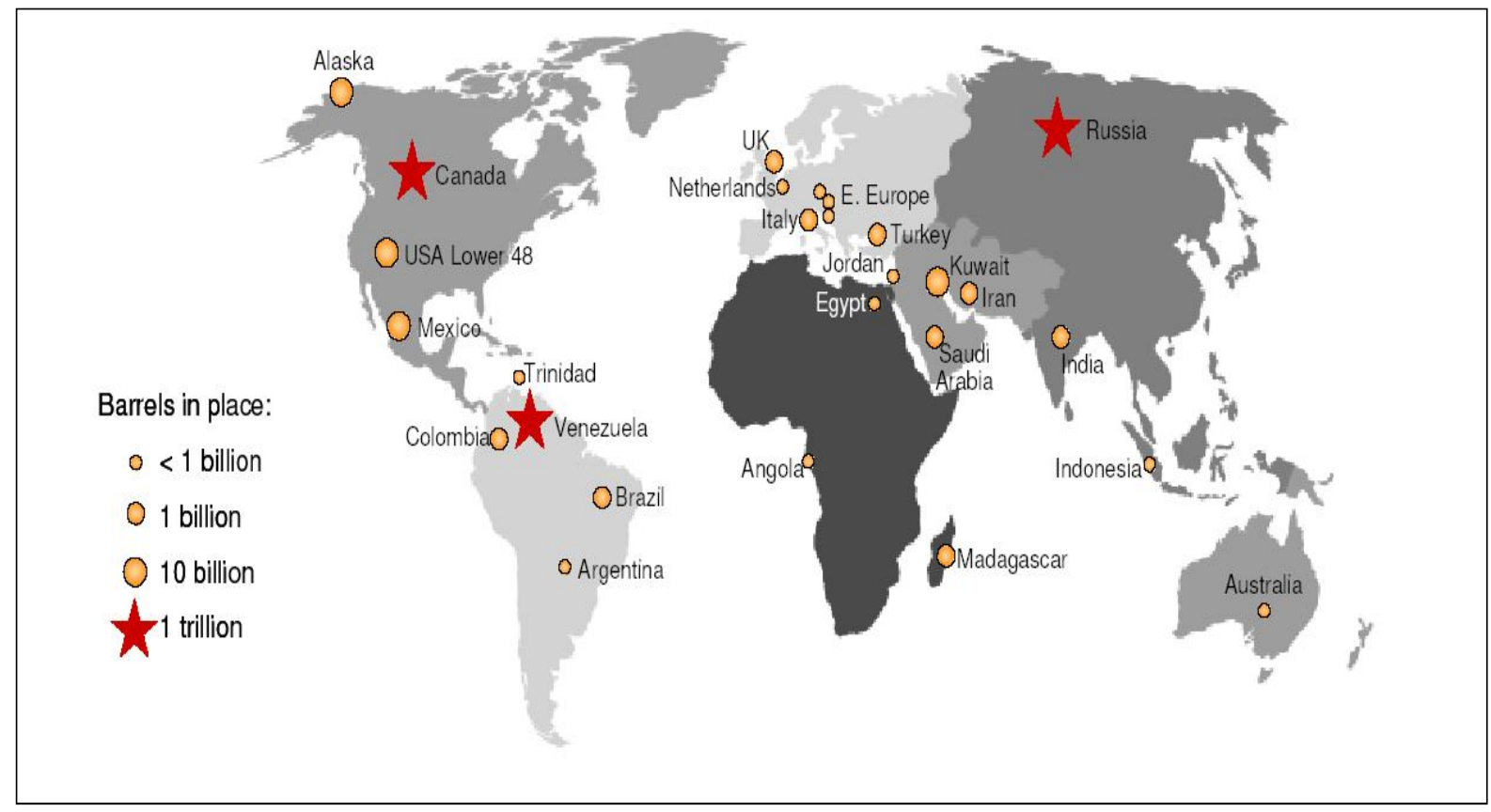

Figure 1.3 Countries with significant amounts of bitumen 


\subsection{Canada's Oil Sands}

Canada's oil sands deposits contain as much as 175 billion barrels of economically viable oil. The hydrocarbon reserves exist in the unconsolidated sand and carbonate sedimentary formations. With current technology of enhanced oil recovery, Canada's oil sands reserves are second only to Saudi Arabia in global oil reserves.

More than one third of the crude oil produced in Canada currently comes from the Alberta and Saskatchewan natural bitumen deposits. The three Alberta oil sands areas, - Athabasca, Peace River, and Cold Lake, - contain at least two-thirds of the world's discovered bitumen in place (1.7 trillion barrels) (Figure 1.4). This fact indicates that heavy oil and bitumen reserves have great potential to meet the ever-increasing demands of energy and other petroleum products. Major world producers now target the heavy oil and bitumen reserves.

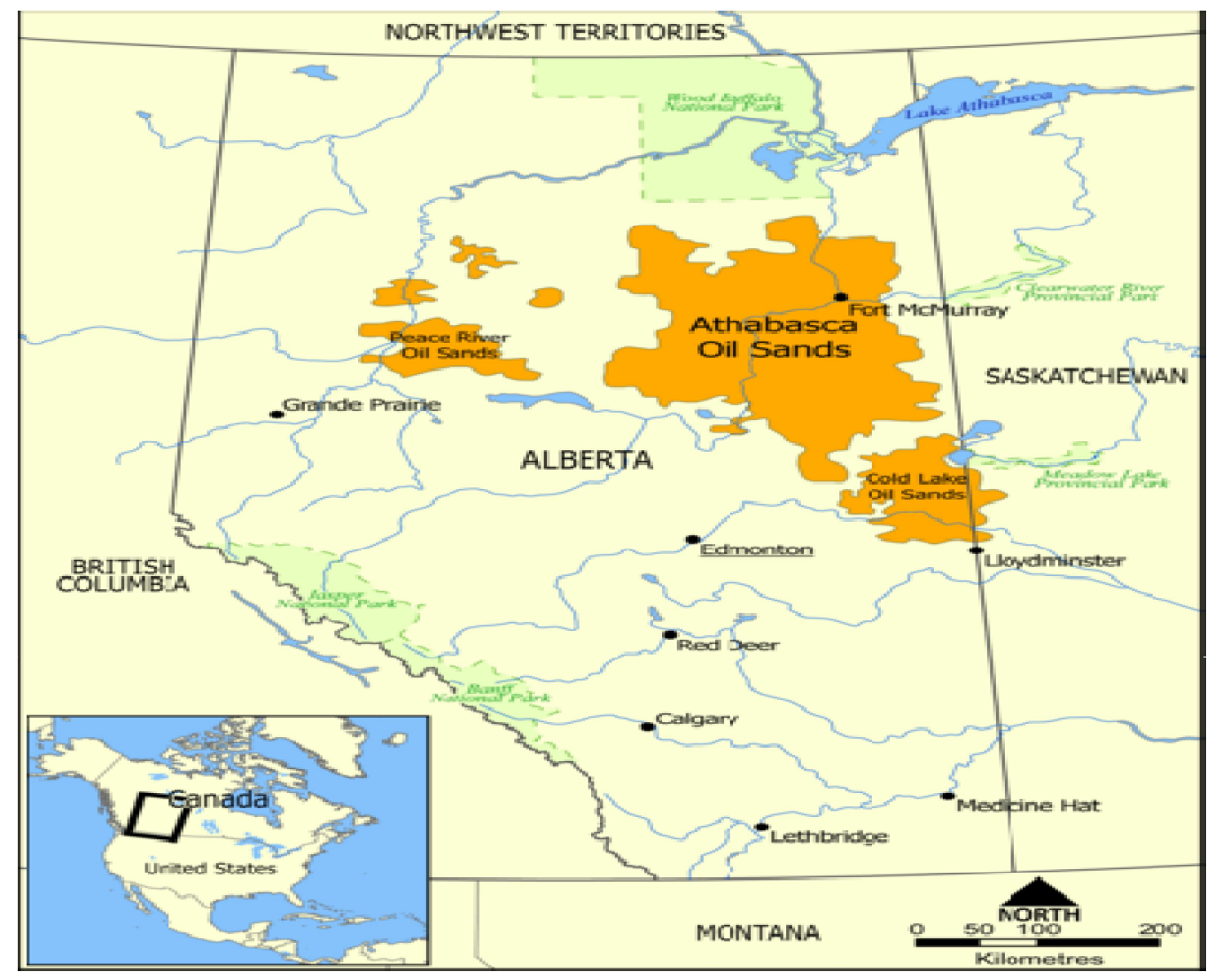

Figure 1.4 Canada's heavy oil and bitumen (Wikimedia 2006) 
Compared to conventional crude oil recovery, the recovery of heavy oil and bitumen reserves is a challenging task. Conventional oil flows at reservoir temperature and can be pumped from the ground, but oil sands must be mined or recovered in-situ. Surface mining or open pit mining is a process that involves digging up the oil sand reserves lying close to the surface $(<75 \mathrm{~m})$, then transporting it to an extraction facility where it is washed with hot water and bitumen is separated from the sand leaving a huge volume of sand to be disposed of.

The majority of the heavy oil and bitumen lie below the surface of 400 meters or more, and can be extracted through in-situ techniques. The objective of all in-situ recovery processes is to reduce the viscosity or to increase the mobility of bitumen while lies underground. This can be achieved by providing additional heat or materials to heavy oil and bitumen reserves to reduce the viscosity.

In-situ recovery processes can be classified into two categories: thermal and non-thermal. Thermal processes use heat to reduce the viscosity of the heavy oil and bitumen in-place, thus mobilizing the heavy oil and bitumen. Examples include cyclic steam stimulation (CSS), steam assisted gravity drainage (SAGD), in-situ combustion, and steam flooding. Steam assisted gravity drainage (SAGD) (Butler and Mokrys, 1989) is one of the most commonly used thermal process in Canada. It involves drilling two horizontal wells at the bottom of a thick unconsolidated sandstone reservoir, then injecting steam slowly in the upper horizontal well (injection well) and developing a "steam chamber". The heat and steam increase oils mobility, whereas condensed water and mobilized oil flow downward through the porous medium by gravity driven flow to the lower horizontal well (production well). Despite the higher recovery expected from thermal methods, the huge amount of energy required, higher greenhouse gas emissions, water pollution and much higher production costs result in major drawbacks.

Another category is the non-thermal processes that rely on solvent dilution of the oil to reduce the heavy oil and bitumen viscosity. Examples include $\mathrm{CO}_{2}$ injection, miscible floods, and vapor extraction (Vapex) process. 


\subsection{The Vapex Process}

The Vapex technology was proposed by Butler and Mokrys (1989). Over the past years, the process has moved from the concept to field scale pilot tests. The Vapex process as shown in Figure 1.5 utilizes two horizontal wells as in the Steam Assisted Gravity Drainage (SAGD) process, but with the steam chamber replaced by a light hydrocarbon vapor chamber. It is important to mention that mass transfer theories use "solute" and "solvent" terminologies in a different way than that used in oil industry. In mass transfer theories, solvent is referred to heavy oil and solute to vapor (gas). However, in this work solvent is referred to vapor (gas).

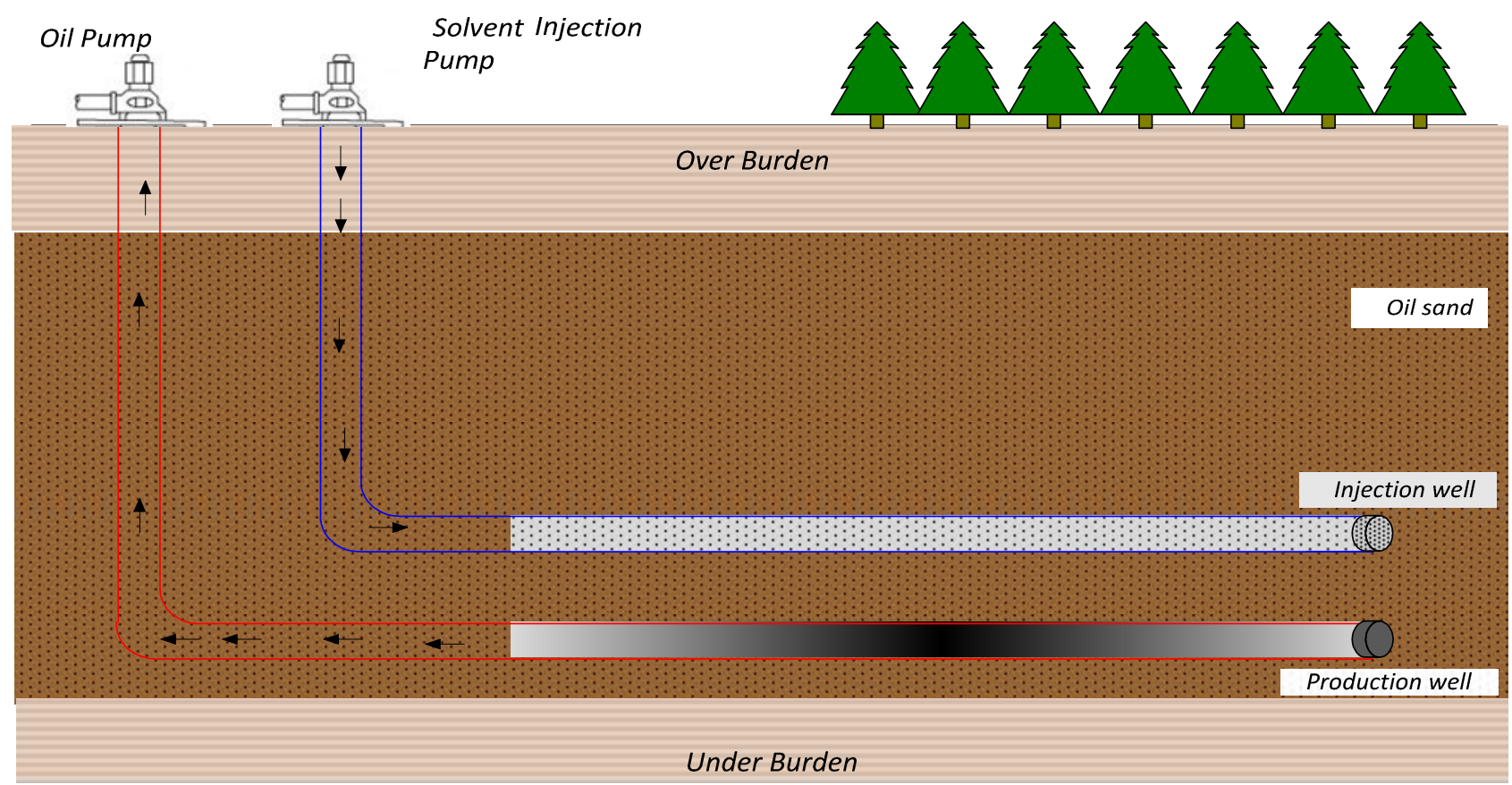

Figure 1.5 Side view of Vapex

In (Vapex), vaporized solvents are injected into the upper horizontal well. As the solvent diffuses into the viscous oil, a significant reduction in viscosity is experienced making it mobile enough to drain downward to the production well. It is now referred to as live oil. Diluted oil drains along the solvent vapor oil interface under the force of gravity to the production well. Many solvent gases have been considered such as propane, butane, and mixtures of both. The choice of solvents based on several factors: saturation vapor pressure, molecular weight, density 
difference, solubility and diffusivity. Mokrys and Butler (1993) have shown experimentally that the optimum solvent injection condition is near the solvent dew point where the solvent has the maximum solubility and diffusivity in the heavy oil. Since the reservoir pressure is much higher than the solvent dew point at the reservoir temperature, a non-condensable carrier gas usually injected with the solvent to raise the dew point pressure and keep the solvent in a vapor phase. Carbon dioxide, nitrogen and methane can be used as a carrier gas.

Figure 1.6 illustrates the Vapex process and the growth of the solvent chamber. The solvent rises up to reach the top of the formation resulting in a vapor chamber above the injection well then the chamber starts to spread laterally towards the outer boundary over time. During this spreading phase, the flow rates are stabilized. When the solvent interface reaches the formation boundary, the gravity head for drainage decreases, then the production rate declines.

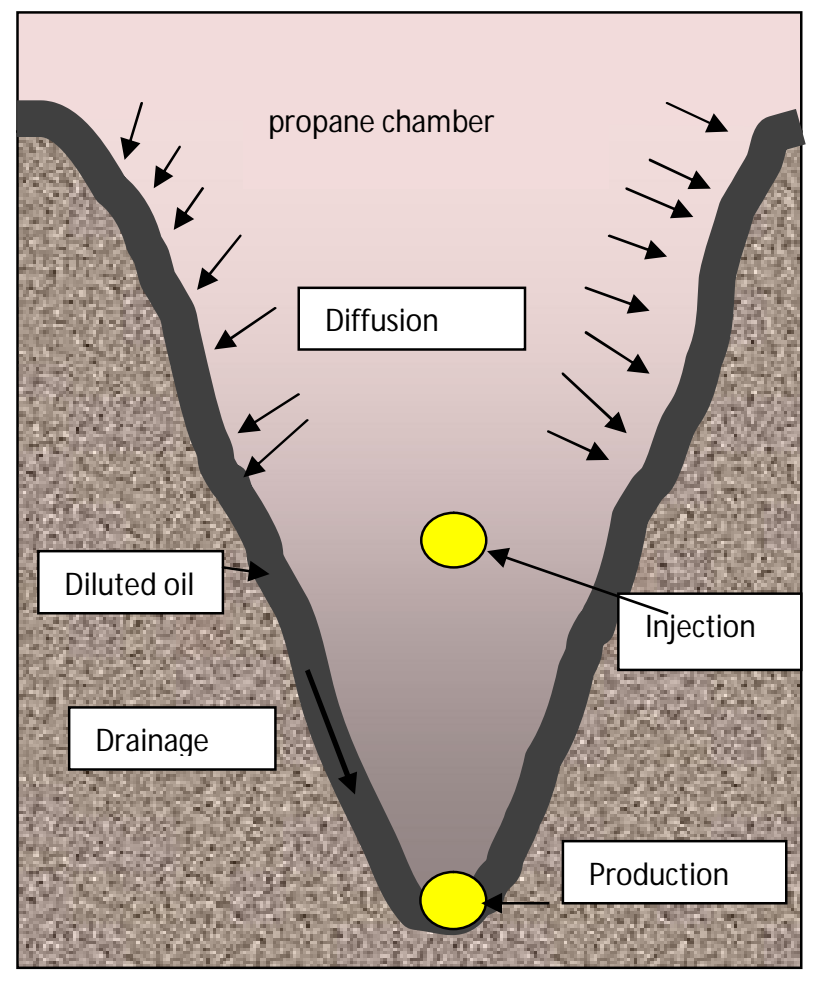

\section{Figure 1.6 Cross sectional view of Vapex}

The production rate is governed by two mass transfer mechanisms: molecular diffusion and mechanical (convectional) dispersion taking place at the solvent-heavy oil interface. Gravity is 
the driving force for the live oil flow in Vapex process since the pressure difference between the two horizontal wells is small.

Vapex can be used as an alternative to recover the heavy oil and bitumen from reservoirs, which are not suitable for thermal processes such as reservoirs with bottom water, vertical fracture, thin pay zones, and low thermal conductivity. The implementation of Vapex cuts down green house gases (GHG) emission by $80 \%$ compared to other thermal processes (Das, 2002). For the same production rate Vapex process uses only 3\% of the energy required by steam processes (Das, 1998). In addition, Vapex has low capital costs, as the injection of vaporized solvents does not require extensive surface facilities. Furthermore, injection of the solvent vapor close to its dew point can cause in-situ upgrading of the produced oil by asphaltenes precipitation (Mokrys and Butler 1993). In addition, carbon dioxide sequestration is possible with Vapex, thus reducing the amount released to the atmosphere. Moreover, the solvent is recoverable which reduces the operating cost.

Although production rates with this process are lower than with traditional steam processes, Vapex promises to improve energy efficiency and to reduce emissions and operating costs. One of the advantages of the Vapex process is the in-situ deasphalting that is inherent with solvent extraction. Das (1995) observed that the asphaltene precipitation requires an operating pressure greater than the vapor pressure of the solvent being used at a given temperature. Luo et al. (2008) has observed in Vapex tests that when the operating pressure is too close to the vapor pressure of pure propane or the dew-point of a butane mixture, asphaltene precipitation occurs and it strongly depends on the sand-packed permeability. Some researchers concluded that deasphalting does not stop oil from being produced but enhances the oil flow rate due to the viscosity reduction (Das and Butler, 1994). In contrary, other researchers (Mokrys and Butler, 1993, Haghighat and Maini, 2008) found that asphaltene precipitation reduced the permeability and the oil production rates.

\subsection{Dispersion}

The fluid spreading phenomenon through a system (e.g. a porous medium) is called dispersion. It is the combined effect of the two physical phenomena, molecular diffusion and convection 
caused by fluid velocity gradients. Consider the example of a slug of tracer introduced into a flowing water stream in a capillary tube (Figure 1.7). At any point, both diffusion and the velocity of the fluid transport the tracer. If the velocity within the tube is uniform, the slug spreads and is mixed by molecular diffusion only (Figure 1.7a). However, if the flow within the tube is fully developed steady laminar flow, the velocity profile is parabolic in the radial direction and the slug spreads out following the shape of the velocity profile. If there is no molecular diffusion, the slug will keep spreading according to the parabolic profile (Figure 1.7b). If diffusion is present, it will equalize concentrations in the radial and axial directions and at sufficient distance, the tracer concentration becomes uniform in the radial direction (Figure 1.7c). Therefore, spreading of the slug is a result of the interplay between the convective velocity profile and molecular diffusion and it is termed dispersion (Taylor 1953; John 2008).

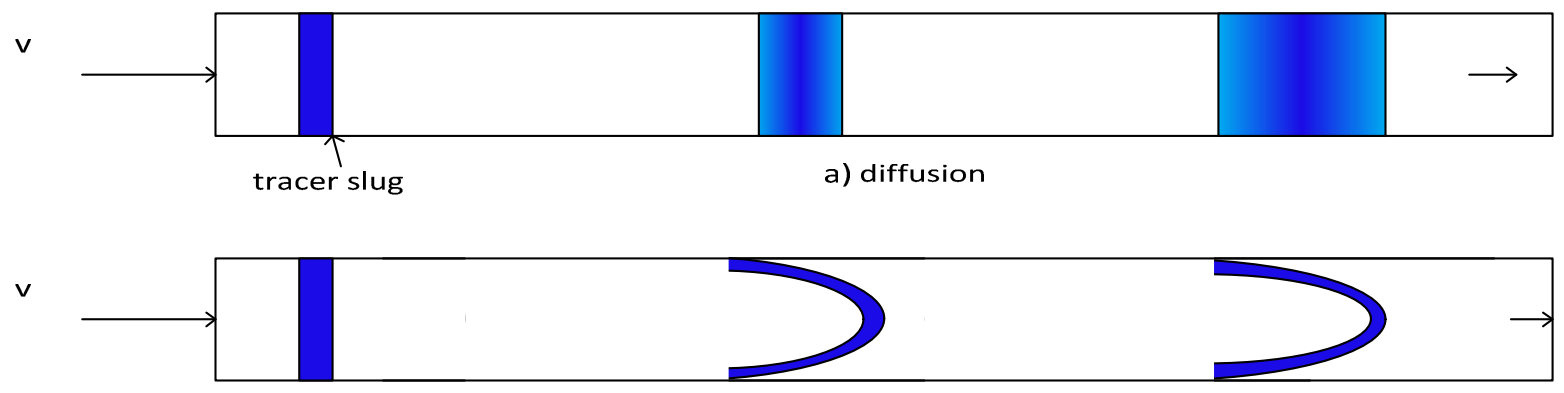

b) convection

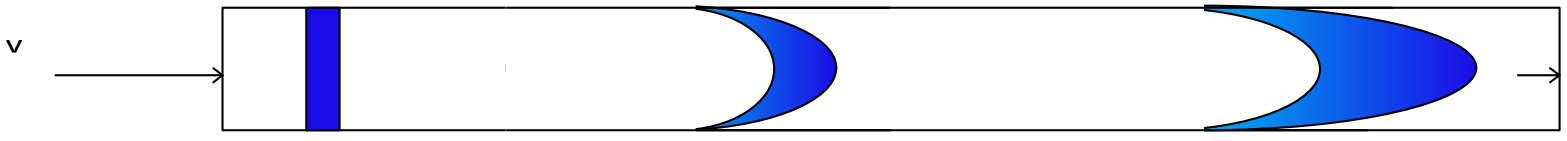

c) convection-diffusion

Figure 1.7 Sketch showing diffusion, convection and dispersion in a capillary tube (John, 2008)

Dispersion plays a significant role in a variety of transport processes. The oil recovery efficiency of processes like miscible gas or chemical flooding depends on the mixing of the injected gas or chemical. Modeling the transport processes requires an accurate estimation of the amount of dispersion and its impact relative to other transport mechanisms. 


\subsection{Optimal Control Theory}

Optimal control theory is an optimal method to determine a control policy for a given system that will maximize or minimize a specific performance criterion subject to constrains describing the system dynamics. Optimal control has applications in many different fields, including aerospace, process control, and engineering. Only simple optimal control problems were solved before the 1950s. The revolution of the digital computers in the 1950s allows the application of optimal control theory and methods to complex problems. Many applications of optimal control theory to enhanced oil recovery methods were developed. For example, the optimization of surfactant flooding process, micellar/polymer process, and miscible carbon dioxide process (Ramirez, 1987). Although only initial studies are present, promising advances are expected in the application of optimal control theory to EOR processes.

A branch of mathematics that is useful in solving optimal control problems is the calculus of variations (Denn 1969; Kirk 1970; Ray 1981). Calculus of variations deals with functionals, or functions whose independent variables are functions themselves. To solve optimal control problems where the objective is to determine a function that minimize or maximize a specified functional, calculus of variations is a useful technique.

The analogous problem in calculus is to determine a point that yields the minimum or maximum value of a function. The variation plays the same role in determining extreme values of functionals as the differential does in finding maxima and minima of functions. The fundamental theorem used in finding extreme values of a function is the necessary condition that the differential vanishes at an extreme point. In variational problems, the analogous theorem is that the variation must be zero on an extremal.

For example, functionals can be formed as integrals involving an unknown function and its derivatives:

$J(x)=\int_{t_{o}}^{t_{f}} g(x(t), \dot{x}(t), t) d t$ 
It is desired to find the function $x^{*}$ for which the functional $J(x)$ has a relative extremum. $J$ is a functional of the function $x$, and $g(x(t), \dot{x}(t), t)$ is a function assigns a real number to the point $(x(t), \dot{x}(t), t)$. It is assumed that $t_{o}$ and $t_{f}$ are fixed, and the end points of the curve are specified as $x_{o}$ and $x_{f}$. The curve $x^{*}$ extremize $J(x)$. Determination starts by finding the variation $\delta J(x, \delta x)$ and applying the fundamental theorem which yields

$\delta J\left(x^{*}, \delta x\right)=0=\int_{t_{o}}^{t_{f}}\left\{\frac{\partial g}{\partial x}\left(x^{*}(t), \dot{x}^{*}(t), t\right)-\frac{d}{d t}\left[\frac{\partial g}{\partial \dot{x}}\left(x^{*}(t), \dot{x}^{*}(t), t\right)\right]\right\} \delta x(t) d t$

Thus, the integral must be zero, and a necessary condition for $x^{*}$ to be an extremal is

$\frac{\partial g}{\partial x}\left(x^{*}(t), \dot{x}^{*}(t), t\right)-\frac{d}{d t}\left[\frac{\partial g}{\partial \dot{x}}\left(x^{*}(t), \dot{x}^{*}(t), t\right)\right]=0$

for all $t \in\left[t_{o}, t_{f}\right]$. Differential equations of this type are hard to solve analytically and numerical integration should be used.

To extend the necessary conditions derived above to include problems with constraints, the Lagrange multipliers are introduced to form the augmented functional by adjoining the constraining relation to $J$ which yields:

$J(x, p)=\int_{t_{o}}^{t_{f}}\{g(x(t), \dot{x}(t), t)+p(t)[f(x(t), t)]\} d t$

where $f(x(t), t)=0$ is the constraint relation. On an extremal the variation must be zero, that is, $\delta J\left(x^{*}, p\right)=0$, and the constrained must be satisfied by an extremal. The variation of $J$ yields:

$$
\begin{gathered}
\delta J(x, \delta x, p, \delta p)=\int_{t_{o}}^{t_{f}}\left\{\left[\frac{\partial g(x(t) \dot{x}(t), t)}{\partial x}+p(t) \frac{\partial f(x(t), t)}{\partial x}\right] \delta x(t)+\left[\frac{\partial g(x(t), \dot{x}(t), t)}{\partial \dot{x}}\right] \delta \dot{x}(t)+\right. \\
[f(x(t), t)] \delta p(t)\} d t
\end{gathered}
$$

Integrating by parts the term containing $\delta \dot{x}$ yields: 


$$
\begin{gathered}
\delta J(x, \delta x, p, \delta p)=\int_{t_{o}}^{t_{f}}\left\{\left[\frac{\partial g(x(t), \dot{x}(t), t)}{\partial x}+p(t)\left[\frac{\partial f(x(t), t)}{\partial x}\right]-\frac{d}{d t}\left[\frac{\partial g(x(t) \dot{x}(t), t)}{\partial \dot{x}}\right]\right] \delta x(t)+\right. \\
[f(x(t), t)] \delta p(t)\} d t
\end{gathered}
$$

The variation must be zero on an extremal. The constraint must be satisfied by an extermal, we can choose $p$ such that the coefficient of $\delta x$ is zero through the interval $\left[t_{o}, t_{f}\right]$. Thus, the equation:

$\frac{\partial g\left(x^{*}(t), \dot{x}^{*}(t), t\right)}{\partial x}+p^{*}(t)\left[\frac{\partial f\left(x^{*}(t), t\right)}{\partial x}\right]-\frac{d}{d t}\left[\frac{\partial g\left(x^{*}(t), \dot{x}^{*}(t), t\right)}{\partial \dot{x}}\right]=0$

must be satisfied. Equation (1.7) and the constraint relation, $f(x(t), t)=0$, form a set of necessary conditions for $x^{*}$ to be an extremal. This technique was used to derive necessary conditions for determination of optimal solvent dispersion function in heavy oil for Vapex experiments.

This work investigates the use of optimal control theory to find out solvent dispersion function in vapor extraction process of heavy oil. To solve an optimal control problem, we must first describe the problem in physical terms, and then translate the physical description into mathematical terms. Once the optimal problem is defined mathematically, we can apply the optimal control theory to the partial differential equations describing the process model.

\subsection{Research Objectives}

The main drawback of the Vapex process is the lower oil production rates compared to SAGD process. The oil production rate is governed by the dispersion phenomena, which is a combination of molecular diffusion and convective mixing. Thus, the knowledge of dispersion of solvents in heavy oil and bitumen is crucial to optimize the oil production rates. With dispersion data, engineers can determine the amount of solvent required for injection and the time taken to achieve the optimal recovery of heavy oil and bitumen. In addition, the knowledge of the effect of operating parameters on dispersion data such as drainage height and porous medium 
permeability will allow engineers to determine the optimal pay zones depth and numbers and locations of injection and production wells.

The above considerations coupled with the paucity of dispersion data in the literature make it imperative to determine the dispersion coefficients for various solvents in heavy oil and bitumen. For this purpose, the objectives of this research study were defined as follows:

1. Conduct Vapex experiments using lab physical models of different permeabilities and heights utilizing propane as a solvent to produce adequate experimental data.

2. Develop a new technique that is based on optimal control theory in conjunction with a developed mass transfer model of the experimental Vapex process.

3. Develop a computational algorithm to compute optimally solvent-heavy oil interface mass fraction and solvent dispersion as a function of its concentration in heavy oil.

4. Investigate the effects of laboratory model permeability and height on both the value of the solvent dispersion coefficient and live oil properties.

\subsection{Structure of the Dissertation}

Following is the outline of this dissertation:

- Chapter 1: A general description of the Vapex process, list of research objectives, and organization of the text are introduced.

- Chapter 2: Literature review is provided on Vapex process and both diffusion and dispersion phenomena. In addition, experiments and numerical simulations on dispersion determination is included.

- Chapter 3: The experimental methods and procedures are explained in this chapter.

- Chapter 4: The mass transfer model describing the Vapex process and the mathematical framework to determine the concentration-dependent dispersion of the solvent in heavy oil is developed in this chapter. It includes a set of necessary optimality conditions and the computational algorithm.

- Chapter 5: The experimental and numerical simulation results are presented, analyzed, and discussed in details.

- Chapter 6: The contribution of this research is summarized. The areas of the future work and recommendations are presented. 


\section{Literature Review}

This chapter reviews the history of Vapex, the concept of diffusion and dispersion in porous media, and propane dispersion evaluation in Vapex.

\subsection{History of Vapex}

The idea to use solvents to recover heavy oil and bitumen can be traced back in the seventies, when Allen (1974) injected propane or ethane in cycles to extract Athabasca bitumen in a packed model. Allen and Redford (1976) injected liquid solvent along with non-condensable gas in the reservoir. Nenniger (1979) found out that heavy oil and bitumen can be recovered by injecting the reservoir with solvent gas or a mixture of solvent gases at or below their vapor pressure. All these processes use vertical injection and production wells. However, the observed uneconomical production rates prevented or delayed the implementation of these methods in the field.

Later, Butler et al. (1981) used parallel horizontal wells to recover steam-heated heavy oil by gravity drainage in a process named Steam Assisted Gravity Drainage (SAGD). The horizontal wells allow larger contact area between the steam and the heavy oil, which result economical production rates. The economical production rates allowed the implementation of SAGD in the field. Butler and Mokrys (1989) extended the SAGD concept to the solvent extraction processes by introducing a solvent analog of SAGD process to recover heavy oil and bitumen. They attempted to extract the bitumen using liquid toluene in a Hele-Shaw cell. Butler and Mokrys (1991) replaced the liquid solvent with gaseous solvent and introduced the vapor extraction of heavy oil (Vapex) technique using a packed cell and propane as a solvent. 
Since 1989, extensive experimental studies on Vapex have been performed using laboratory scale physical models. The physical models can be non-porous models (Hele-Shaw cells) or porous models with various geometry (unconsolidated sand or glass beads) ( Butler and Mokrys 1991; Das and Butler 1996; Jin 1999; Jiang and Butler 1996; Oduntun 2001; Karmaker and Maini 2003; Friedrich 2005; Yazdani and Maini 2005; Moghadam et al. 2009).

\subsection{Diffusion and Dispersion Phenomena}

The performance of Vapex is directly related to the amount of solvent dissolving into the heavy oil. Solvent transfers into the heavy oil by the transport mechanism of dispersion. Dispersion is a combination of molecular diffusion and convective dispersion. Knowledge about these mechanisms in Vapex is scarce, in particular the magnitude of solvent dispersion coefficient. In the following sections, the related literature and work will be reviewed.

\subsubsection{Molecular Diffusion}

The transport of molecules from one location to another due to a gradient in their concentration is defined as molecular diffusion. Molecular diffusion is the result of random motion of molecules. The process occurs until the concentration at the two locations becomes equal i.e until the concentration gradient ceases to exist. The diffusion of molecules in gases is well known and can be estimated (Reid et al. 1987). The diffusion of molecules in liquids is less well known, especially at reservoir conditions. Many investigators tried to generalize predictive correlations based on theoretical approaches such as hydrodynamic theory, kinetic theory, and absolute rate theory. However, none of these theories is satisfactory in predicting the actual value of diffusivities in heavy oil and bitumen because of the assumptions involved.

Hayduk and Cheng (1971) tested the hypothesis that diffusivity of a particular species in any solvent depends on the solvent viscosity. The following relationship between the diffusivity and viscosity was proposed:

$$
D=\alpha \mu^{-\beta}
$$


where $\alpha$ and $\beta$ are constants for each solute/solvent pair. Many predictive correlations have been suggested (Boustani and Maini, 2001). Each correlation is valid only at its relevant assumptions. Thus, if the predictive correlations fail to give reliable estimates, experimental methods can be used to determine the molecular diffusion of solvent in heavy oil and bitumen.

Experimental methods for the determination of diffusivity of solvents in bitumen can be classified into direct/conventional and indirect/unconventional methods. Direct methods are based on determination of composition of the diffusing species along the length of the bitumen sample with time and require compositional analysis. On the other hand, the indirect methods measure the change in one of the system parameters that varies because of the diffusion, without determining the composition. Such parameters are pressure, interface position, magnetic field strength, or the volume of the diffusing solute.

In the presence of porous medium, an apparent diffusion coefficient is defined based on the average cross-sectional area open for diffusion. Perkins and Johnston (1963) related the porous medium effect to the formation electrical resistivity, which is a function of porosity. Das (1995) modified molecular diffusion in porous medium by introducing the cementation factor $\Omega$. He related the molecular diffusion $D_{o}$ to apparent diffusion coefficient $D_{\text {app }}$ in porous medium by the following relationship:

$D_{\text {app }}=D_{o} \varphi^{\Omega}$

where $\Omega$ is a cementation factor, which is a measure of consolidation of rock. For the case of unconsolidated rocks, he used 1.3 as a cementation factor.

\subsubsection{Concentration Dependence of Molecular Diffusion}

The molecular diffusion coefficient is not a constant. It varies with temperature, concentration and pressure. In most of the published literature on molecular diffusion measurements in hydrocarbons, the calculations are based on the assumption that the diffusion is constant over the concentration range used in the experiments. However, Oballa and Butler (1989) studied the 
diffusion process in toluene-bitumen system and found that the overall diffusion coefficients were strongly dependent on the concentration of the solute. The results are shown in Figure 2.1.

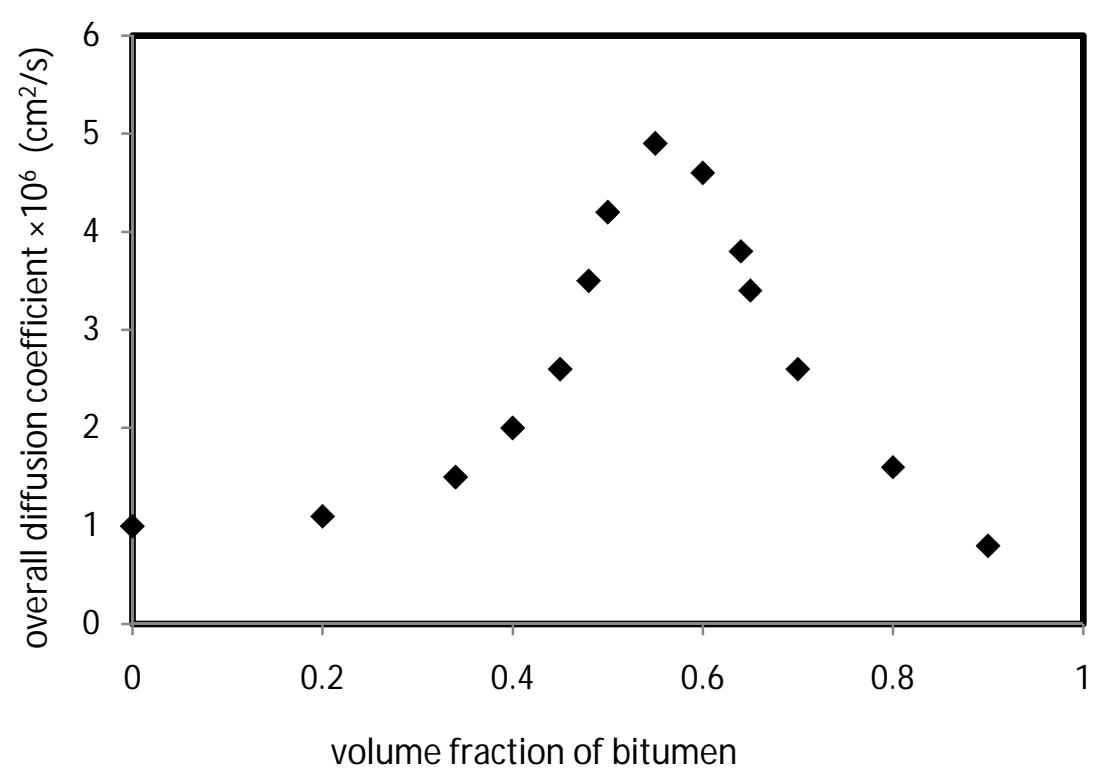

Figure 2.1 Overall diffusion coefficient for toluene-bitumen system (after Oballa and Butler, 1989).

Butler and Mokrys (1989) also found similar results for toluene-Athabasca system as shown in Figure 2.2. 


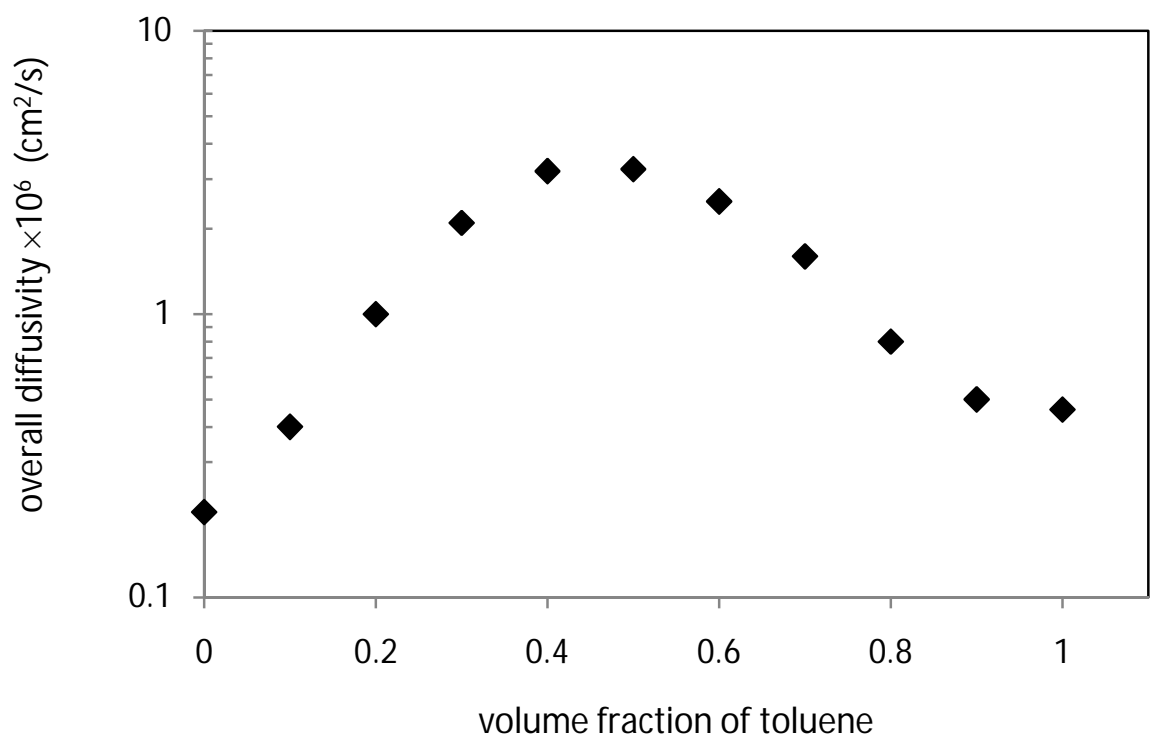

Figure 2.2 Concentration dependence of diffusivity (after Butler and Mokrys, 1989).

Das and Butler (1996) obtained empirical correlations for the diffusion coefficients of propane and butane in bitumen. The diffusivity showed a strong dependence on viscosity. The dependence of diffusivity on viscosity and the dependence of viscosity on solvent concentration imply that diffusivity in heavy oil is a function of solvent concentration. Upreti and Mehrotra (2000) obtained similar results as the concentration profiles for the sorption of carbon dioxide in Athabasca bitumen indicated that the diffusivity is concentration dependent.

Luo et al. (2007) found that the diffusivity of heptane in heavy oil is concentration dependent. At higher heavy oil mass fractions (0.53-1), a linear dependence of diffusivity on heavy oil was obtained as shown in Figure 2.3. In addition, Luo and Kantzas (2008) obtained the same dependence of diffusivity on solvent concentration for heptane-heavy oil system in sand packs. 


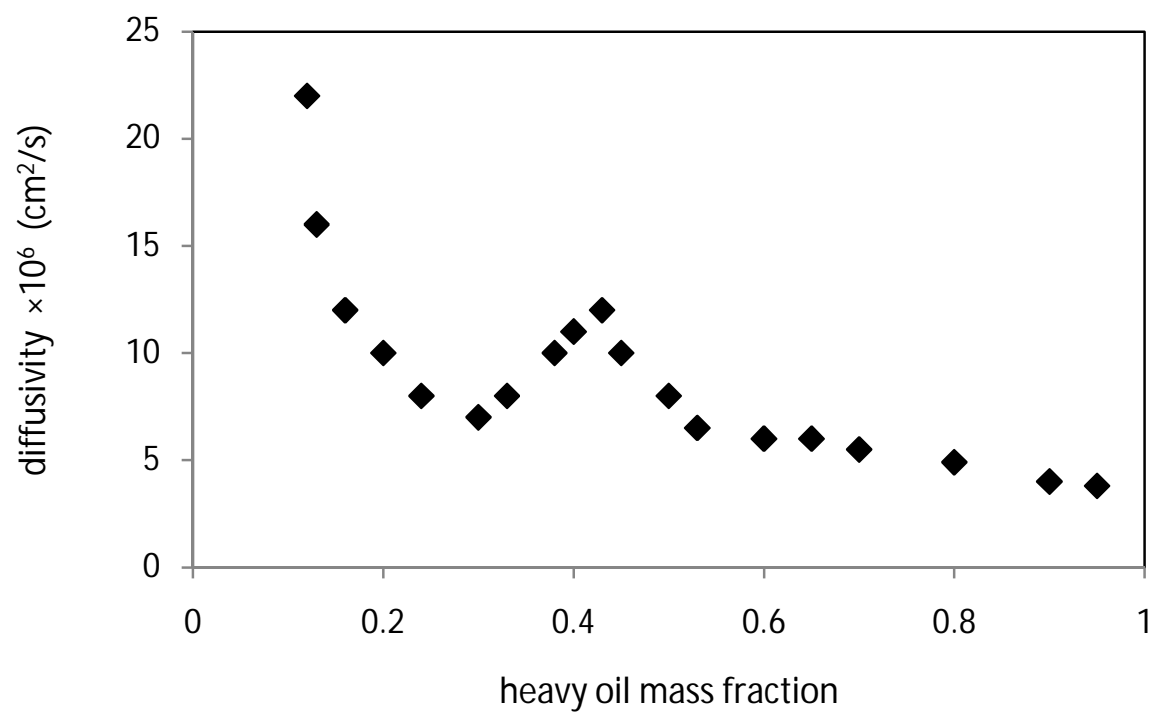

Figure 2.3 Concentration dependence of diffusivity (after Luo et al., 2007).

Earlier estimates based on a mass transfer model, which is only comprised of molecular diffusion alone, were unable to explain the observed high recovery rates in Vapex (Dunn et al. 1989; Das and Butler 1998; Boustani and Maini 2001; Oduntan et al. 2001). Investigators reported that up to four orders of magnitude higher molecular diffusion values were needed to match the experimental production rates with the predicted ones. They related this increase to dispersion phenomenon, which is a combination of molecular diffusion, mechanical (convection) dispersion, and other mechanisms that promote mixing such as capillary action, imbibition, surface renewal and the action of gravity (Das and Butler, 1998).

\subsubsection{Dispersion in Porous medium}

Dispersion is the macroscopic outcome of the actual movements of a component in a phase through the pores and the various physical and chemical phenomena that take place within the pores. There are two basic transport phenomena involved in the dispersion process: molecular diffusion and mechanical (convection) dispersion. Mechanical (convective) dispersion is defined by spreading or mixing of a component in a phase caused by microscopic variations in flow velocity in a porous medium (Bear, 1972). 
Mechanical dispersion in porous medium is comprised of two components: the longitudinal and transverse dispersion. The longitudinal dispersion is parallel to the direction of bulk flow of mixture, and the transverse is perpendicular to the direction of the bulk flow of mixture. In the longitudinal case, the mechanisms related to dispersion include fluid velocity gradients within the individual pore spaces and molecular diffusion. In the transverse case, the mechanisms related are the intertwining of flow paths and molecular diffusion. Greenkorn (1983) provided details of the mechanisms involved in porous medium that can affect the magnitude and direction of velocities, which in turn influence in mechanical dispersion.

Dispersion coefficients can be determined by experimental methods and analyses as described by several authors (Perkins and Johnston 1963; Brigham 1961). Perkins and Johnston (1963) reported the relationships for longitudinal and transverse dispersion as the sum of diffusion and convective dispersion terms and the relations were as follows:

$\frac{K_{l}}{D_{o}}=\frac{1}{F \varphi}+0.5 \frac{U \sigma d_{p}}{D_{o}} ; \quad \frac{U \sigma d_{p}}{D_{o}}<50$

$\frac{K_{t}}{D_{o}}=\frac{1}{F \varphi}+0.0157 \frac{U \sigma d_{p}}{D_{o}} ; \quad \frac{U \sigma d_{p}}{D_{o}}<10^{4}$

where $K_{l}$ and $K_{t}$ are the longitudinal and transverse dispersion coefficient respectively, $d_{p}$ is the particle diameter, $\sigma$ is the inhomogenetity factor, $D_{o}$ is the molecular diffusion coefficient, $U$ is fluid velocity in the direction of bulk flow, $F$ is a formation factor and $\varphi$ is the porosity of the system. The longitudinal and transverse mixing takes place in porous media due to molecular diffusion is one of the phenomena contributing to dispersion. When there is no flow, molecular diffusion is the only phenomenon contributes to dispersion. In Perkins and Johnston correlations the main driving force is the forced convection and a negligible density difference and a unity viscosity ratio are considered for fluids, which is not the case for Vapex.

The overall mass transfer flux can be written as the sum of convective, diffusion, and mechanical dispersion. Convective flux is the flux of solvent carried by the average velocity of the flowing fluid. Mechanical dispersion flux is the flux of the solvent due to the spreading of the solvent in 
the fluid flowing (streamlines fluctuating) by the pores of the porous medium. Diffusion flux is the flux of the solvent due to molecular diffusion. Analogous to Fick's first law of diffusion, the dispersive flux of a component in porous medium can be expressed as (Bear and Buchlin, 1991):

$J_{\text {disp }}=-\varphi D_{\text {disp }} \nabla c$

where $\nabla c$ is the solvent concentration gradient. The dispersion coefficient, $D_{\text {disp }}$, has contributions of both molecular diffusion and mechanical (convective) dispersion. Thus, the two coefficients (diffusion and convective dispersion) can be summed up as:

$J_{\text {disp }}=-\varphi\left(D_{o}+D_{\text {conv }}\right) \nabla c$

where $D_{\text {conv }}$ is the convective dispersion coefficient and $D_{o}$ is the molecular diffusion coefficient.

\subsection{Dispersion in Vapex}

Several authors used diffusion in their mathematical model to predict the oil production rate in vapor extraction of heavy oil and bitumen. However, they found that the predicted production rate was lower than experimental one. Hence, they claimed that dispersion is necessary to predict the actual production rate.

Dunn et al. (1989) proposed and tested a gravity drainage concept for in situ recovery of bitumen by mobilization with soluble gases at ambient reservoir temperature conditions. Experiments were performed using Carbon dioxide and ethane gases at $20^{\circ} \mathrm{C}$ to recover Athabasca bitumen from a scaled model. They modified the thermal gravity drainage for the mass transfer case. They found that the measured drainage rates were higher than those predicted by the model using molecular diffusivity. Thus, to match their experimental results using the theoretical model, they used an effective diffusivity, two to three orders of magnitude higher than the reported molecular diffusivity. They pointed out the possibility of increased recovery due to dispersion.

Das and Butler (1998) conducted series of experiments in a sand pack with Peace River and Lloydminster bitumen and butane gas as solvent. They found that experimental production rate 
was significantly higher than the predicted value from the modified analytical model. They suggested that several factors contributing to recovery rate enhancement in porous media. These factors are: extended interfacial area, increased rate of solubility, capillary action and surface renewal. They realized that the effective diffusion, 3 to 10 times higher than molecular diffusion, is required to match their experimental results with analytical model.

Mechanical (convective) dispersion was pointed by Lim et al (1996) as one of the most viable processes governing the recovery of heavy oil and bitumen in solvent assisted gravity drainage process. They produced diluted bitumen applying the concept of a single horizontal well cyclic process for three-dimensional scaled sand packs injected with ethane. They used 2-3 orders of magnitude higher than molecular diffusivity to history-match their experimental results.

Boustani and Maini (2001) studied the role of diffusion and convective dispersion in Vapex by using a Hele-Shaw Cell, and incorporated the results in a predictive model. The incorporation of dispersion effects into a Vapex mass transfer model showed good agreement between theory and experiment. In their work, the overall mass transfer in Vapex process was modeled in 2D space for longitudinal and transverse dispersion.

Das (2005) considered a 2D field scale simulation model using CMG's GEM simulator. He studied the rate of mixing between injected solvent and in-situ oil using different diffusion coefficients. Very high coefficients (order of $10^{-2}-10^{-3} \mathrm{~cm}^{2} / \mathrm{s}$ ) were needed to match production rates, while only coefficients of $10^{-5} \mathrm{~cm}^{2} / \mathrm{s}$ produced the interface profile expected i.e drainage rates and interface profile cannot be matched simultaneously using a single diffusion coefficient.

As mentioned above, predictions of recovery rates based on only molecular diffusion failed to explain the high recovery rates observed in packed physical models in Vapex experiments. Therefore, at least a secondary mechanism as convective dispersion should be incorporated into a mass transfer model to address the observed high recovery rates in porous medium.

Several researchers have attempted to extract the related Vapex diffusion and dispersion coefficients by conducting experiments using different techniques as CT scan and MRI imaging 
or by developing mathematical models or numerically simulating the Vapex process and history matching the Vapex experiments (Fisher et al. 2000; Nghiem and Kohse 2001; Karmaker and Maini 2003; James et al. 2003; Cuthiell et al. 2003; Wen and Kantzas 2005).

Kapadia et al. (2006) developed and simulated a mathematical model with a linear concentrationdependent dispersion to determine gas dispersion during the vapor extraction of Cold Lake bitumen from a rectangular block of homogeneous porous medium using butane. The dispersion coefficient was found to be four orders of magnitude higher than reported molecular diffusion. The concentration-dependent dispersion of butane in Cold Lake bitumen dispersion was determined as

$D=5.56 \times 10^{-5} \omega\left(\mathrm{m}^{2} / \mathrm{s}\right)$

where $\omega$ is the mass fraction of butane in Cold Lake bitumen.

Using a linear dispersion model, El-Haj et al. (2009) conducted Vapex experiments, which were simulated by a mathematical model to determine the dispersion coefficient of butane gas into Athabasca bitumen. The dispersion coefficient obtained was two to three orders of magnitude higher than molecular diffusivity reported earlier. Notably, it was observed that the dispersion coefficient was higher for lower permeability physical models.

Yazdani and Maini (2009) introduced a new correlation for the effective diffusion/dispersion coefficients of Vapex experiments with physical models of varying sizes and different permeability sand-packs using butane as solvent. The correlation accounted for the drainage height dependency and the concentration dependency, which embodied in the solvent-heavy oil mixture viscosity. Each attempt has its own simplifying mathematical assumptions and limitations on the experimental method.

\subsection{Diffusion and Dispersion of Propane in Vapor Extraction of Heavy Oil}

In the literature, there are limited data for propane diffusion and dispersion coefficients in heavy oil and bitumen although the diffusion and dispersion data of propane is an important parameter 
for the reservoir simulation and field design of Vapex process. A few attempts were carried out to measure propane diffusion coefficient in heavy oil and bitumen. Hayduk et al. (1973) measured the diffusivities of propane in dilute solutions of $n$-butanol and normal paraffins of hexane, heptane, octane, and hexadecane at different temperatures using the steady state capillary cell method.

Das and Butler (1996) used a Hele-Shaw cell to obtain empirical correlations for the diffusivities of propane in Peace River bitumen as a function of propane-bitumen mixture viscosity, which in turn is a function of propane concentration. Their results indicate that propane diffusivity increases with its concentration in bitumen. From zero to unit volume fraction of propane, it is estimated to increase monotonically from $0.2 \times 10^{-9}$ to $0.9 \times 10^{-8} \mathrm{~m}^{2} / \mathrm{s}$.

Using the pressure decay method, Tharanivasan et al. (2006) determined the diffusion coefficients of propane in heavy oil for three different boundary conditions at the propane-heavy oil interface. They used a constant diffusion model developed with an analytical solution and optimized to history match the results of other authors. The best history matching of the calculated pressure with the measured data for propane was obtained by applying the quasiequilibrium boundary condition at the propane-heavy oil interface at $\mathrm{T}=23.9^{\circ} \mathrm{C}$.

Yang and Gu (2006) developed a new method named dynamic pendant drop volume analysis (DPDVA) to measure the diffusion coefficient of propane in heavy oil in the pressure range of 0.4-14.0 $\mathrm{MPa}$ and at $\mathrm{T}=23.9^{\circ} \mathrm{C}$. They developed a mathematical model with two different boundary conditions and assumed constant diffusivity. The diffusivity of propane in LIoydminister heavy oil at $\mathrm{T}=23.9^{\circ} \mathrm{C}$ and in the pressure range of $0.4-0.9 \mathrm{MPa}$ was in the range of $0.09 \times 10^{-9}-0.68 \times 10^{-9} \mathrm{~m}^{2} / \mathrm{s}$.

However, the results do not present propane diffusion or dispersion coefficient in Vapex process in the presence of porous medium. Therefore, a few investigators attempted to measure propane effective diffusion or dispersion coefficient in heavy oil using sand or glass packed models to simulate the vapor extraction process in real reservoirs. 
Lim et al. (1996) used Cold Lake oil sand scaled physical model with sand porosity $\varphi=0.328$ and permeability $\mathrm{K}=80$ Darcy to produce bitumen by horizontal well cyclic propane recovery process. The experiments conducted at $33^{\circ} \mathrm{C}$ and 0.83-1.0 MPa. Effective diffusion coefficient of propane in heavy oil was estimated using the analytical model of Butler and Mokrys (1989) by assuming a constant diffusion coefficient over the solubility range. Their results indicated that the effective diffusivity of propane was in the range of $2.7 \times 10^{-8}-7.5 \times 10^{-8} \mathrm{~m}^{2} / \mathrm{s}$.

Nghiem et al. (2001) carried out reservoir simulation runs of a field scale Vapex process in the compositional simulator for Lindbergh oil and propane mixture to study the effect of the dispersivity coefficients on the mixing process. They incorporated the dispersion coefficient in their model to investigate the effect of dispersion and diffusion. Several runs were performed with different values of dispersivity $(0.25 \mathrm{~m}, 0.5 \mathrm{~m}$, and $1.00 \mathrm{~m})$ and with $\varphi=0.34$, horizontal permeability $\mathrm{K}=9000 \mathrm{md}$, and vertical permeability $\mathrm{K}=4500 \mathrm{md}$ at $\mathrm{T}=21{ }^{\circ} \mathrm{C}$ and $\mathrm{P}=858 \mathrm{kPa}$. The average values for the convective dispersion coefficients $(\propto|u|)$ for $\propto=0.25$ is around $1.4 \times 10^{-7}$ $\mathrm{m}^{2} / \mathrm{s}$, with values reaching $8 \times 10^{-7} \mathrm{~m}^{2} / \mathrm{s}$. They pointed out that molecular diffusion was important in the start up phase when the oil flow velocity is small, once the oil flow was established; convective dispersion became the main driving force for mixing. However, the reliability of the results depends on the magnitude and correctness of the dispersivity values employed in the simulation since dispersivity is a scale dependent parameter.

Ramakrishnan (2003) measured the effective diffusion coefficient of propane in heavy oil using a rectangular channel model packed with glass beads saturated with heavy oil. He conducted the experiments at different permeabilities (25, 86, 220 Darcy) and different dip angles (45, 75, 80, 90) with a porosity of 0.38 . The Butler's gravity drainage equation was applied assuming the dependence of viscosity and diffusivity on solvent volume fraction to determine the diffusion coefficient based on the experimental oil production rates. The correlation developed of the diffusion coefficient of propane was:

$D_{\text {eff }}=1.85 \times 10^{-9} \mu^{-0.9}$

where $D_{\text {eff }}$ is the effective diffusivity. 
Kramar and Maini (2003) have used magnetic resonance images (MRI) to extract the dispersion coefficients of propane as solvent into Athabasca bitumen during a laboratory-scale Vapex process. The magnitudes of dispersion coefficients were obtained by non-linear regression of the MRI signal strength with the aid of a mass transfer model incorporating estimated interfacial velocities and a constant dispersion coefficient. They used the mathematical model to analyse 2D MRI images captured during a Vapex process published by Fisher et al. (2000) to extract the dispersion coefficient of propane in bitumen. The physical model was a rectangular sand pack model saturated with Athabasca bitumen. The experiments were carried out at $17{ }^{\circ} \mathrm{C}$ average temperature. The net dispersion coefficient $\left(D_{n e t}=D_{t} \cos \theta-D_{l} \sin \theta\right)$ of propane in bitumen in the horizontal direction was in the range of $1.53 \times 10^{-10}-1.18 \times 10^{-9} \mathrm{~m}^{2} / \mathrm{s}$, where $D_{\text {net }}$ is the net dispersion coefficient, $D_{t}$ is the transverse dispersion coefficient, $D_{l}$ is the longitudinal dispersion coefficient, and $\theta$ is the inclination angle of the interface with the vertical axis. Their model considered the dispersion to be independent of solvent concentration and the mass transfer model was treated as a semi-infinite mass transfer model which was found to be erroneous (Schmidt et al., 1982).

Das (2005) investigated the sensitivity and usefulness of the prediction of the oil production rates using different orders of magnitude of diffusion and dispersion coefficients of propane in a two dimensional simulation model. Very high coefficients (order of $10^{-2}-10^{-3} \mathrm{~cm}^{2} / \mathrm{s}$ ) were needed to match production rates. He also reported that in Vapex process, the solvent does not have the opportunity to penetrate very far inside the bitumen. As soon as it reaches enough mobility, it drains down and the chance of developing a Fician concentration gradient is very slim. Under those conditions a higher mass transfer rate would produces a higher extraction rate.

As mentioned above, studies in the literature assumed a uniform value for propane dispersion coefficient and overlooked the concentration dependence of dispersion. However, constant values can be assumed in cases of dilute solutions, not in the case when a light hydrocarbon diffuses into heavy oil to reach an average concentration of 0.3-0.44 mass fraction. In addition, the analytical mass transfer models utilized neglected the convection and the mechanical dispersion terms. Other studies have used empirical correlations developed from experiments 
with vastly different mechanisms. Therefore, the determined propane dispersion values may not represent the dispersion phenomena occurring in Vapex and can lead to unrealistic results.

Table 2.1 Details of studies on the determination of propane dispersion/effective diffusivity in Vapex

\begin{tabular}{|c|c|c|c|c|}
\hline Reference & Heavy Oil & Oil viscosity & $\begin{array}{c}\text { Operating } \\
\text { conditions }\end{array}$ & $\begin{array}{c}\text { Dispersion/ effective } \\
\text { diffusivity }\left(\mathrm{m}^{2} / \mathrm{s}\right)\end{array}$ \\
\hline Lim et al.(1996) & Cold Lake & $80,000 @ 25^{\circ} \mathrm{C}$ & $33^{\circ} \mathrm{C}, 0.83-1.0 \mathrm{M} \mathrm{Pa}$ & $\mathrm{D}_{\text {eff }}=2.7 \times 10^{-8}-7.5 \times 10^{-8}$ \\
\hline $\begin{array}{c}\text { Ramakrishnan } \\
(2003)\end{array}$ & Athabasca & $70,000 @ 23^{\circ} \mathrm{C}$ & $22^{\circ} \mathrm{C}, 0.761 \mathrm{M} \mathrm{Pa}$ & $\mathrm{D}_{\text {eff }}=1.85 \times 10^{-9} \mu^{-0.9}$ \\
\hline $\begin{array}{c}\text { Nghiem et al. (2001) } \\
\text { Kramar and M aini } \\
(2003)\end{array}$ & Lindbergh & $10,000 @ 21^{\circ} \mathrm{C}$ & $21^{\circ} \mathrm{C}, 0.858 \mathrm{M} \mathrm{Pa}$ & $D_{\text {conv }}=0.8 \times 10^{-6}$ \\
\hline Das (2005) & Bitumen & N/A & $17^{\circ} \mathrm{C}$ & $D_{\text {net }}=1.53 \times 10^{-10}-1.18 \times 10^{-9}$ \\
\hline
\end{tabular}

The preceding review reveals the scarceness of reliable propane dispersion data of Vapex process in the literature, which makes it crucial to determine the concentration-dependent dispersion for propane in heavy oil and bitumen. In general, determination of the dispersion coefficient of a solvent vapor as a function of its mass fraction in heavy oil and bitumen will allow design and control engineers to model and simulate the amount of solvent required to reduce the oil viscosity and mobilize it for drainage under gravity, and to predict the rate of oil recovery from the oil sands (Upreti et al., 2007).

\subsection{Approach of this Work}

This work is composed of both experimental and modeling-optimal control parts to determine the concentration-dependent dispersion coefficient of propane in heavy oil of Vapex process. A series of experiments are carried out with different physical model drainage heights and glass beads sizes. The properties of propane-heavy oil system are obtained experimentally to provide reliable data for the simulation. 
A mathematical model is developed to describe the mass transfer process based on the vapor extraction experiments of heavy oil using propane as a solvent. The model has an undetermined concentration-dependent dispersion function. Incorporating this function in the mass transfer model, the calculated mass of oil produced should be equal to its experimental value obtained from the experiments. The necessary conditions are derived based on variational calculus, which concerns with finding the extremal of specified functionals. A computational algorithm is implemented to optimally compute dispersion coefficient as a function of the solvent mass fraction in heavy oil. The effects of packed medium permeability and drainage height on the dispersion coefficient of propane are investigated. Figure 2.4 shows a schematic of methodology. 


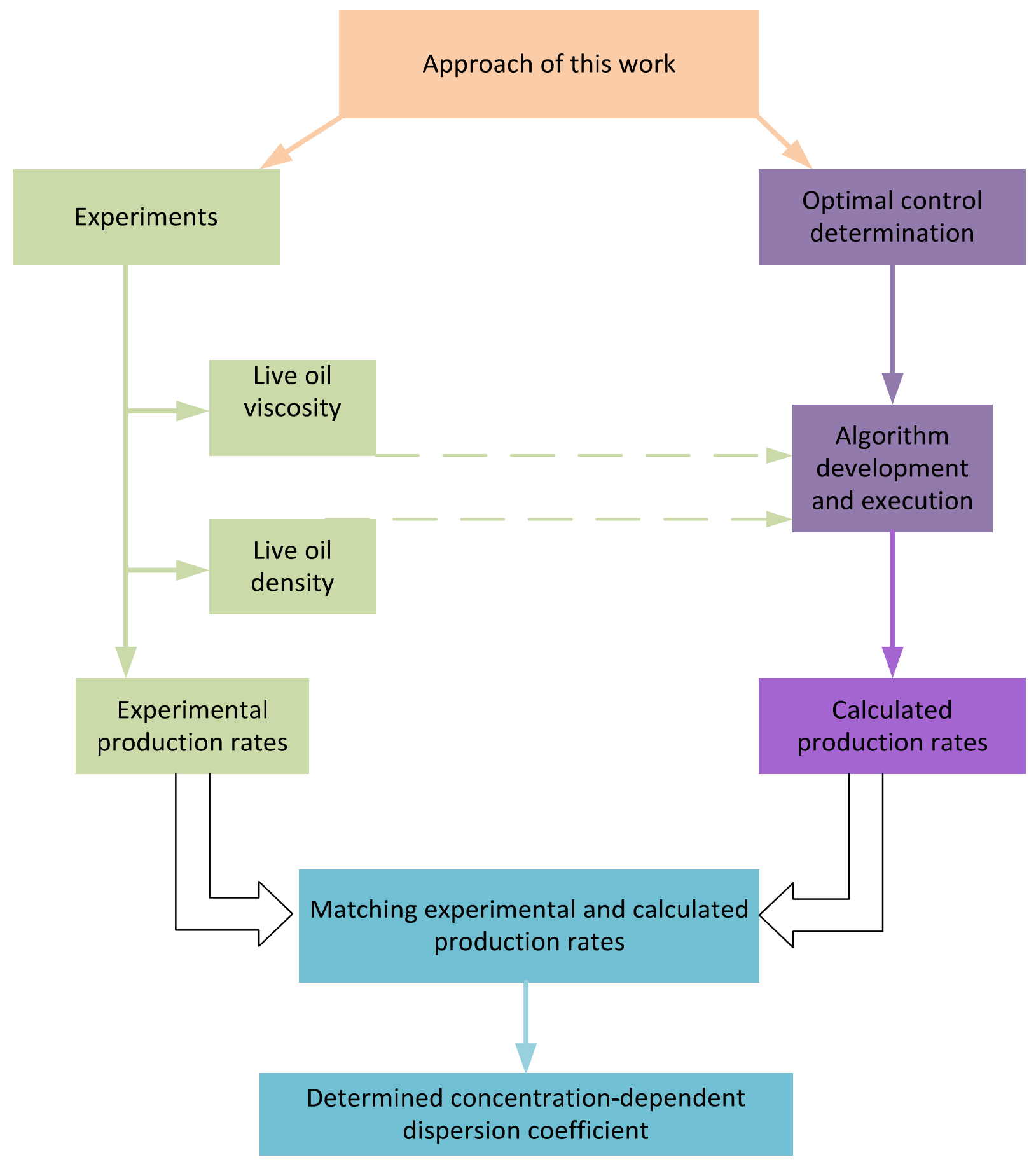

Figure 2.4 Schematic of methodology for this work 


\section{Vapex Experimental Work}

Determination of the concentration-dependent dispersion coefficient of propane relies on data obtained from the Vapex experiments such as the live oil viscosity, density, cumulative production rate, and the amount of propane dissolved in heavy oil. This chapter includes detailed descriptions of the experimental setup, and experimental procedures.

\subsection{Experimental Setup}

The set up used to perform Vapex experiments to determine the dispersion coefficient of propane in heavy oil and bitumen is shown in Figure 3.1 (El-Haj et al., 2009). Three different heights of 25,35 , and $45 \mathrm{~cm}$ cylindrical models with three different permeability of packed medium of 204 , 102, and 51 Darcy were used to examine the effect of height and permeability on propane dispersion. The setup comprises of a cylindrical pressure vessel of $80 \mathrm{~cm}$ height and $15 \mathrm{~cm}$ ID inside a temperature-controlled water bath. The vessel holds the physical model of Vapex. The physical model is suspended from a load cell (LCL-101, capacity: $3500 \mathrm{~kg}, 301 \mathrm{SS}$, provided by OMEGA), and kept in contact with the solvent vapor at constant pressure. The load cell records the mass of the physical model with time. The mass decreases in an experiment as live oil drains away from the physical model located inside the pressure vessel due to solvent dispersion. The drained oil is directed to a calibrated $25 \mathrm{~cm}^{3}$ collection tube. The tube is connected to a viscosity measurement unit to measure the online live oil viscosity.

The viscosity measurement unit comprising of a $0.1016 \mathrm{~cm}$ ID stainless steel capillary tube of length $50 \mathrm{~cm}$ and a differential pressure transducer (250C-P130, supplied by Transicoil Inc. 
U.S.A.). A bypass line was set up to facilitate flow in the event that the capillary tube was plugged. A stainless steel flash separation tank of capacity $300 \mathrm{~cm}^{3}$ wrapped with a flexible electrical heating tape (HTWC101, heat tape with controller supplied by Omegalux) to control temperature around $70^{\circ} \mathrm{C}$. The volume of the gas from the flash tank was measured in a column made of two cylinders attached to each other. The first cylinder of capacity $2,600 \mathrm{~cm}^{3}$ was filled with water. The second cylinder of capacity $2,900 \mathrm{~cm}^{3}$ was used to collect the water displaced from the first column when propane flashed out of the separation tank.

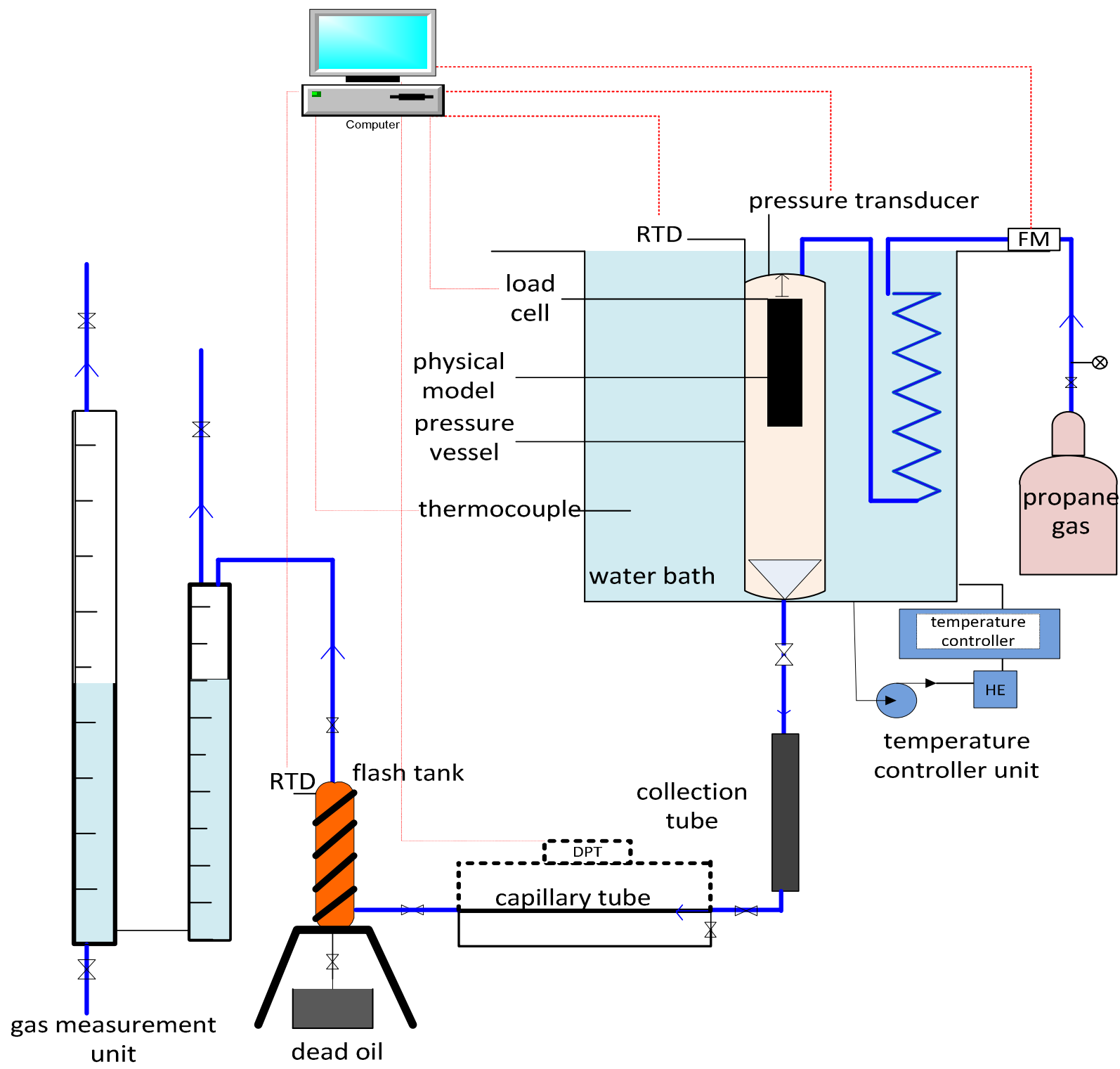

Figure 3.1 Schematic diagram of the experimental setup. 
A vacuum pump was used to evacuate air from the whole setup at the start of each experiment. A temperature controller Fuzypro (F14, PID control mode, supplied by Heaters, Controls And Sensors LTD) was used to maintain the water bath temperature at desired temperature. The temperature of the water bath was measured by a thermocouple (TMTSS, type T, OMEGA). The output of the thermocouple was the input for temperature controller, which was connected to a heat exchanger. The heat exchanger took supply from a water pump placed at the bottom of water tank. A thermocouple (TMTSS, type T, OMEGA) and two resistance temperature detectors (RTD-NPT-72-E high pressure supplied by OMEGA) were used to measure the temperature of the pressure vessel and flash separation tank. The vessel pressure is recorded by a pressure transducer (30-142-30200, series 30 by A-Tech Instruments Ltd). Flow of propane to the pressure vessel was monitored by a flow meter (FMA 1600 A, 2 LPM, supplied by Omega) placed on the supply line of propane.

The experimental conditions were recorded as a function of time automatically by using Ethernet Data Acquisition System - EDAS (16 bit resolution), which was connected to a computer. Labview version 7.1 software provided by National instruments was used for graphical user interface and online monitoring of following inputs: (i) the temperatures of the pressure vessel, water bath, and flash separation tank (ii) pressure in the pressure vessel and the pressure difference across the capillary tube (iii) inlet flow of propane (iv) the mass of the physical model. The sampling time was set to five seconds.

Research grade propane with purity of 99.99\% (MEGS specialty gases Inc., Montreal, Quebec) was used as a solvent at laboratory ambient temperature, which varied between $21^{\circ} \mathrm{C}$ and $22^{\circ} \mathrm{C}$, for all experiments. A photo of the experimental setup is shown in Figure 3.2. 


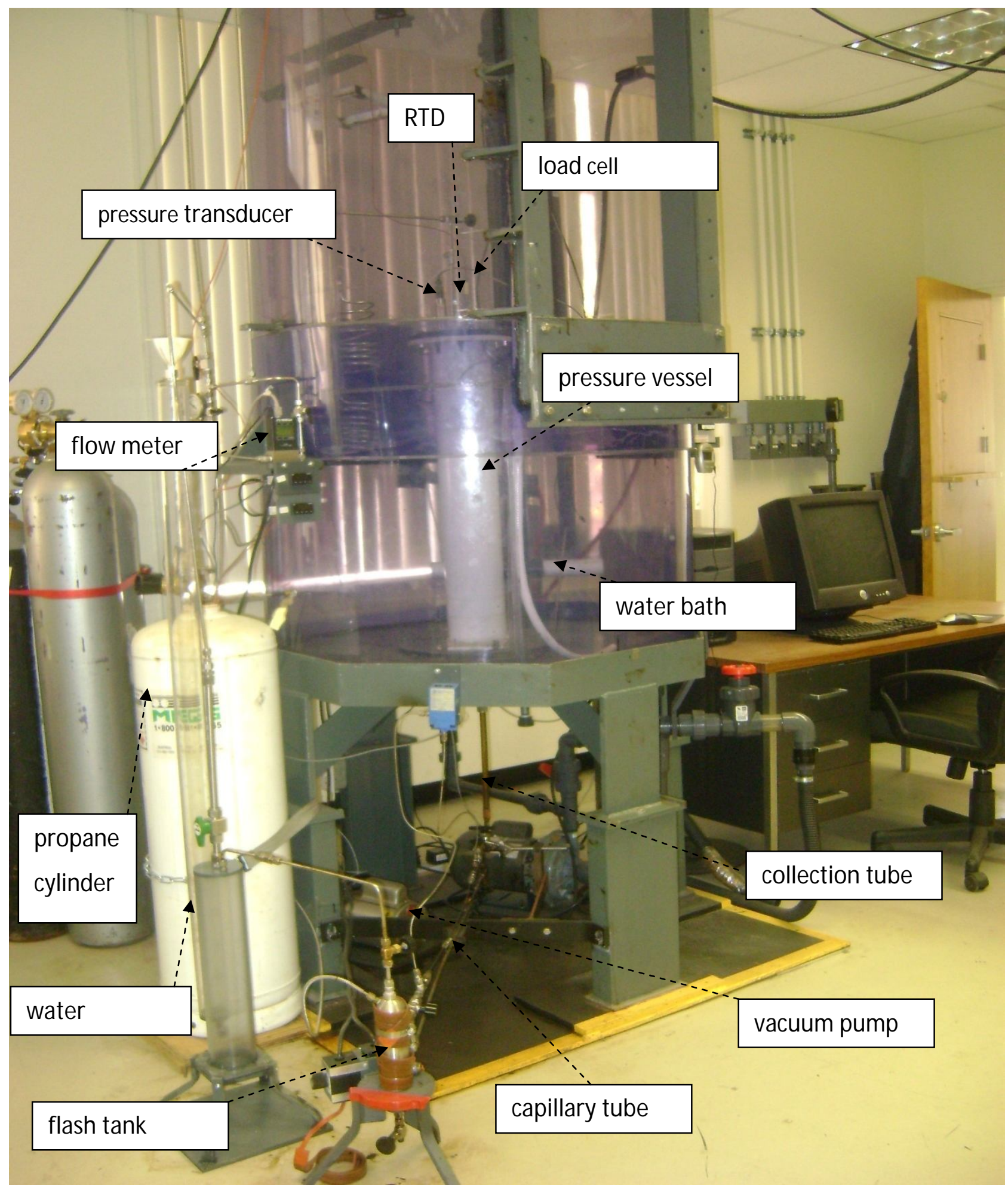

Figure 3.2 Picture of Vapex Experimental Set 


\subsection{Sample Preparation}

Oil-saturated porous medium with glass beads of known permeability was packed in a cylindrical wire mesh of $6 \mathrm{~cm}$ diameter. The oil packing was prepared using a known mass of oil, which was placed in a temperature-controlled heater. The heavy oil was heated for at least 30 minutes at $70^{\circ} \mathrm{C}$, for sufficient reduction in oil viscosity to promote glass beads mixing. The glass beads were slowly poured in the heated oil (in thin layers) and allowed to settle under gravity until the last few stayed on the surface. This method of preparing oil-saturated beads ensured that the glass beads were fully saturated without air being trapped to produce a homogeneous medium. The saturated mixture of heavy oil and glass beads was packed into the cylindrical wire mesh, which was placed inside an ice bath to prevent the oil from oozing out of the mesh. After the entire mesh was packed, it was weighed and left at room temperature for one day to reach thermal equilibrium prior to the experiments.

\subsection{Bitumen Properties}

The heavy oil (Athabasca oil) used in this study was obtained from Imperial Oil Limited. The average bitumen molecular weight according to ASTM standard D2503 was determined to be $557 \mathrm{~g} / \mathrm{mol}$. The density and viscosity of the heavy oil are shown in Table 3.1.

Table 3.1 Properties of the heavy oil

\begin{tabular}{|l|l|}
\hline Property & Value \\
\hline density at $22^{\circ} \mathrm{C}$ & $1.001 \mathrm{~g} / \mathrm{cm}^{3}$ \\
\hline viscosity at $22^{\circ} \mathrm{C}$ & $225,000 \mathrm{CP}$ \\
\hline
\end{tabular}

\subsection{Glass Beads Properties}

The packing material used in this experiment to simulate a reservoir was glass beads obtained from Flex-O-Lite (supplied by Ritchey Supply Ltd.). Specifications of the glass beads are shown in Table 3.2. The particle size distribution curves for the three types of the glass beads used are 
shown in Figure 3.3. From the shape of the distribution curves, the steep slope of the curves indicates that the glass beads have uniform size distribution.

Table 3.2 Specifications of the glass beads

\begin{tabular}{|c|c|c|c|c|c|c|c|c|}
\hline $\begin{array}{c}\text { U.S } \\
\text { Sieve }\end{array}$ & $\begin{array}{c}\text { Common } \\
\text { brand } \\
\text { Flex-0- } \\
\text { Lite }\end{array}$ & $\begin{array}{l}\text { Diameter } \\
\text { Max(mm) }\end{array}$ & $\begin{array}{l}\text { Diameter } \\
\text { Min }(\mathrm{mm})\end{array}$ & $\begin{array}{c}\text { U.S } \\
\text { sieve } \\
\text { size } \\
\text { Min } 95 \\
\text { \% pass }\end{array}$ & $\begin{array}{c}\text { U.S } \\
\text { sieve } \\
\text { size } \\
\text { Max } 10 \\
\text { \% pass }\end{array}$ & $\begin{array}{c}\text { Max } \\
3 \% \\
\text { pass }\end{array}$ & $\begin{array}{c}\text { Min \% } \\
\text { true } \\
\text { spheres }\end{array}$ & $\begin{array}{c}\text { Max \% } \\
\text { sharp } \\
\text { particles }\end{array}$ \\
\hline $30-40$ & BT 4 & 0.594 & 0.419 & 30 & 40 & 45 & 70 & 3 \\
\hline $40-50$ & BT 5 & 0.419 & 0.297 & 40 & 50 & 60 & 70 & 3 \\
\hline $50-70$ & BT 6 & 0.297 & 0.211 & 50 & 70 & 80 & 80 & 3 \\
\hline
\end{tabular}

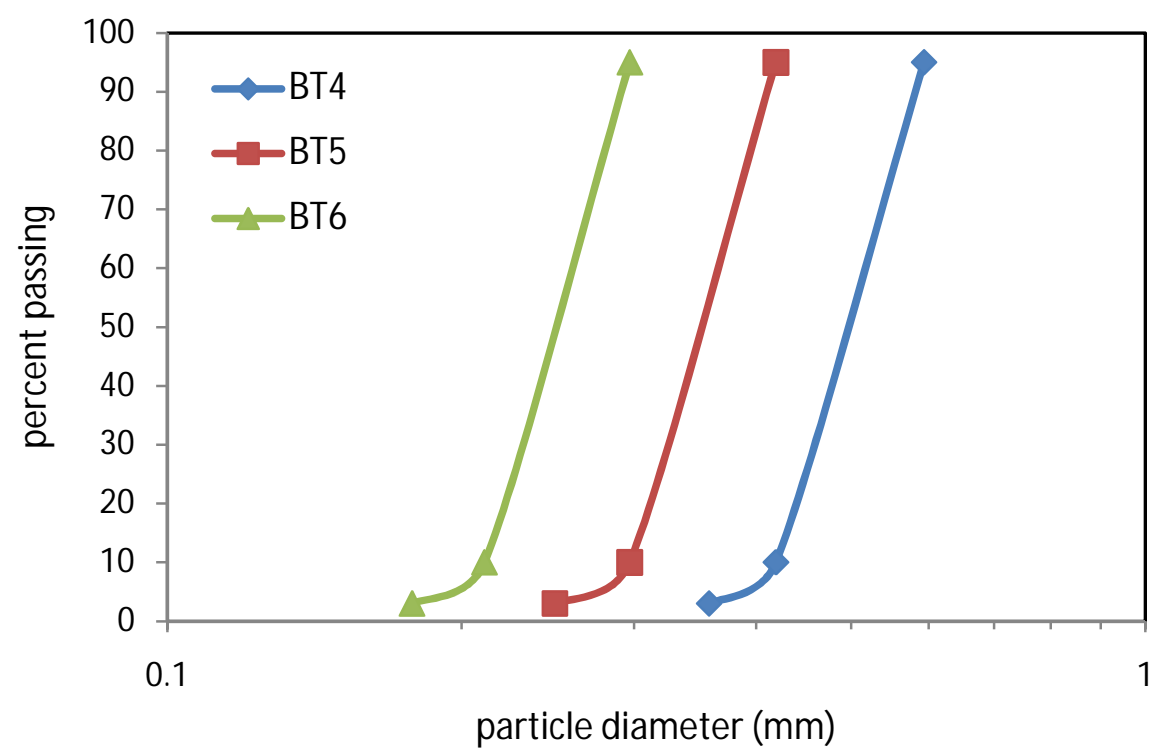

Figure 3.3 Particle size distributions

The permeability of the medium was measured experimentally and estimated using a phenomenological model. The experimental setup used to measure permeability is shown in Figure 3.4. A horizontal cylindrical physical model set up was filled with the glass beads. Air injected into one end (inlet) of the cylinder and exhausted from the other end (outlet). The 
pressure at each end was measured by similar pressure gauges. The airflow rate was measured by a flow meter at the outlet. Darcy law for single-phase steady state flow was applied to calculate the permeability. A sample of the glass beads permeability calculations are presented in Appendix A.

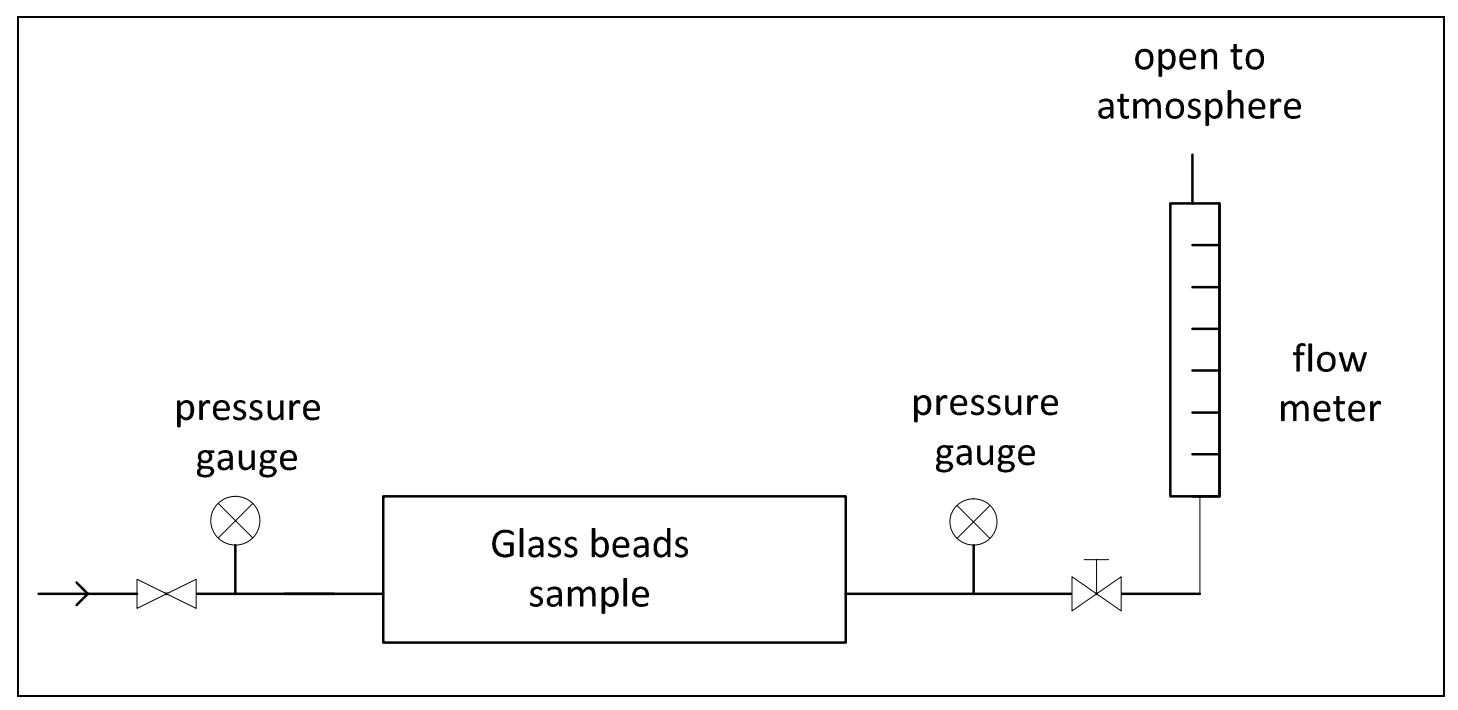

Figure 3.4 Experimental setup for permeability measurement

The permeability of the packing material was also estimated from the particle size diameter utilizing the phenomenological model of Carman-Kozeny:

$K_{\text {Carman-Kozeny }}=\frac{\varphi^{3} D_{p}^{2}}{180(1-\varphi)^{2}}$

Table 3.3 shows measured and estimated permeabilities for the three different glass beads used to pack homogeneous physical models. 
Table 3.3 Permeability of the glass beads

\begin{tabular}{|l|c|c|c|c|}
\hline Glass beads type & $\begin{array}{c}\text { Average diameter } \\
\mathbf{( m m})\end{array}$ & $\begin{array}{c}\text { Estimated using Eqn.(3.1) } \\
\mathbf{K}_{\text {cK (Darcy) }}\end{array}$ & $\begin{array}{c}\text { Experimental } \\
\mathbf{K}(\text { Darcy) }\end{array}$ & Porosity \\
\hline BT 4 & 0.506 & 204 & 222.43 & 0.38 \\
\hline BT 5 & 0.358 & 102 & 114.28 & 0.378 \\
\hline BT 6 & 0.254 & 51 & 66.19 & 0.376 \\
\hline
\end{tabular}

Porosity is a measurement of the fraction of bulk volume occupied by accessible pore space (Dullien, 1992).The Porosity of the packing of different glass beads was measured by the imbibition or saturation method. A cylindrical model was filled with the glass beads and weighed. The cylindrical model was flooded with water from the bottom until it is saturated. The cylindrical model filled with water was again weighed. Porosity of the glass beads in the cylindrical model was determined from the amount of water in the cylindrical model. Table 3.3 shows the measured porosity of the glass beads.

\subsection{Experimental Procedures}

The Leakage test for the pressure vessel was conducted before starting each experiment. The cylindrical pressure vessel was pressurized with air to a pressure of $0.760 \mathrm{MPa}(110 \mathrm{psig})$ and the pressure was detected by a pressure transducer for 12 hours. The pressure transducer detected no air leakage for 12 hours. The cylindrical physical model packed with heavy oil saturated with glass beads was vertically hanged inside the pressure vessel. Air was purged from the entire setup by applying $-15 \mathrm{mmHg}$ reduced pressure using a vacuum pump. To ensure complete displacement of dead air, the pressure vessel was flushed with propane of about twice its volume and reduced to $-15 \mathrm{mmHg}$ pressure. Propane was injected into the vessel at constant pressure of $0.689 \mathrm{MPa}(100 \mathrm{psig})$. The injection pressure was controlled through a pressure regulator installed on the propane supply cylinder. The water bath temperature was kept $3-4^{\circ} \mathrm{C}$ higher than the dew point temperature of propane at the operating pressure (vessel pressure) to ensure that the propane would not condense. The vapor pressure of propane at $21^{\circ} \mathrm{C}$ is $0.755 \mathrm{MPa}(109.5$ psig). The experiment was carried out for $4 \mathrm{hrs}$. 
Propane upon injection diffused into the physical model from its exposed outer surfaces. The heavy oil became less viscous and began to drain into a collection tube as live oil. The load cell recorded the decrease in the mass of the physical model every 5 seconds as the oil production continued. The live oil was collected for the measurement of viscosity and dissolved propane. When about $15 \mathrm{~cm}^{3}$ of live oil was collected, the oil was drained through a capillary tube to measure the live oil viscosity and then into the flash tank. The propane liberated from the live oil in the flash tank was directed to the gas-measurement unit filled initially with water. The displaced volume of water determines the dissolved propane volume. The propane-free oil residual in the flash tank was weighed. At the end of the experiment, the main valve on the propane cylinder was shut and the system depressurized by venting offs the propane into the fume hood. The remaining propane was purged by air to the vent. The model then was taken out of the pressure vessel for visual inspection.

\subsection{Live Oil Viscosity Measurement}

Live oil viscosity was measured experimentally online. The following procedures were implemented as shown in Figure 3.5:

A known volume of live oil was collected in the collection tube. The collected live oil was allowed to flow through the attached capillary tube of $0.1016 \mathrm{~cm}$ diameter and $50 \mathrm{~cm}$ length. A differential pressure transducer recorded the pressure drop across the capillary tube for a given flow rate. A needle valve was used to maintain a constant pressure drop across the capillary tube. Figure 3.6 shows the pressure drop in the capillary tube as a function of time for an experiment. The flow rate of the live oil was determined by measuring the time required to drain a known volume of diluted oil from the collection tube at constant pressure drop. The viscosity of the live oil was calculated using Hagen - Poiseuille equation:

$Q=\frac{\pi \mathrm{d}^{4} \Delta P}{128 \mu L}$

where $\mathrm{d}$ is the diameter of the capillary tube, $\Delta P$ is the pressure drop across the capillary tube, $\mu$ is the live oil viscosity, and $L$ is the length of the capillary tube, and $Q$ is the live oil flow rate. 
A sample of live oil viscosity calculation is shown in Appendix B.

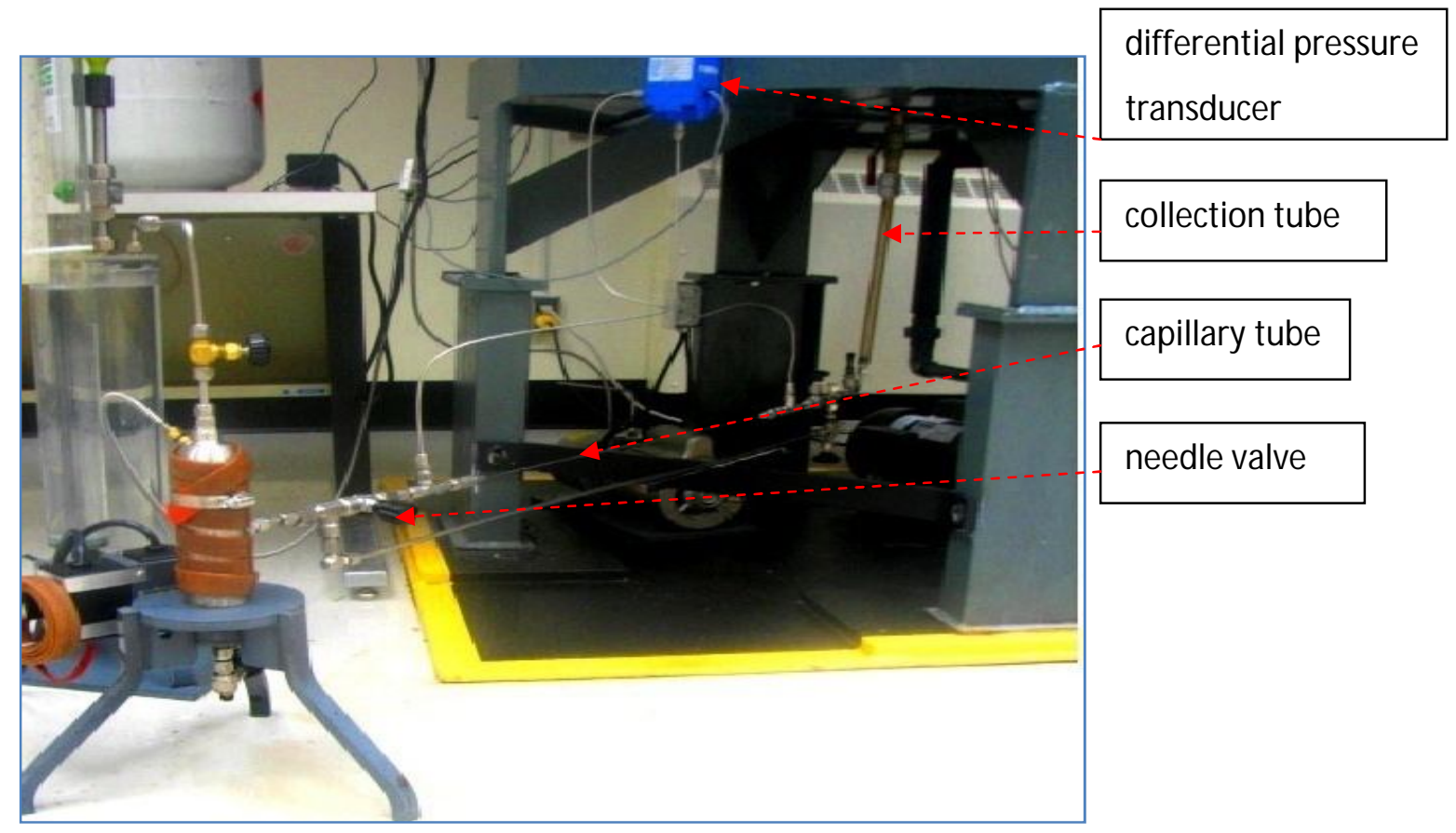

Figure 3.5: Experimental Setup for Live Oil Viscosity Determination

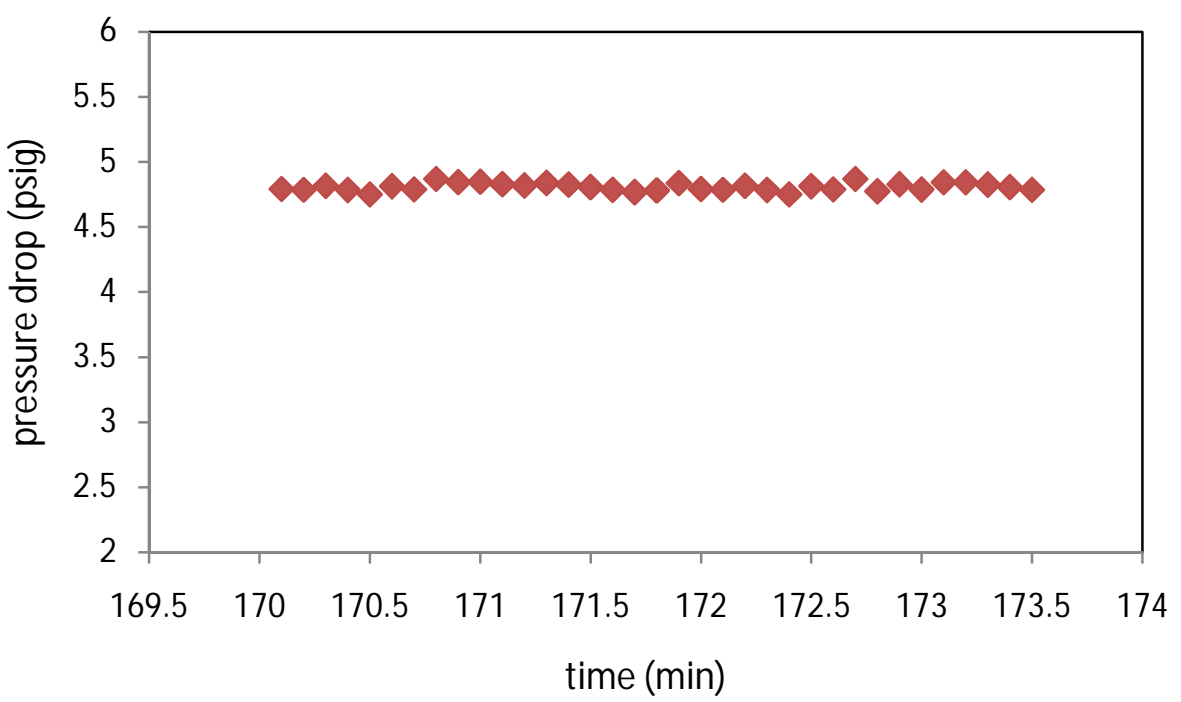

Figure 3.6 Pressure drop across capillary tube versus time as recorded by data acquisition system 


\subsection{Live Oil Density and Amount of Dissolved Propane Measurements}

Amount of dissolved propane in heavy oil was measured experimentally through the following procedures:

A known volume of live oil was allowed to pass through the capillary tube into a flash separation tank. The flash separation tank was wrapped with a flexible heating tape with temperature controller to maintain the temperature inside the separator at $70^{\circ} \mathrm{C}$ or higher to ensure effective flashing of dissolved propane. Sufficient time was given for flashing of propane. The librated propane entered a graduated gas-measuring column. The gas-measuring column was initially filled with water. The displaced volume of water determined the propane volume. The oil residual in the flash tank was collected, weighed, and exposed to the atmosphere for several days. After several days, the oil residual was weighed again and the total volume of released propane was calculated.

Knowing the amount of propane dissolved in oil, the propane-free oil (dead oil) weight, and the volume of the live oil, the solubility of propane was determined as well as the live oil density using the following formulas:

$$
\omega=\frac{\text { mass of liberated propane }}{\text { mass of liberated propane }+ \text { mass of dead oil }}
$$

$\rho_{\text {live oil }}=\frac{\text { mass of liberated propane }+ \text { mass of dead oil }}{\text { volume of live oil }}$

A sample of propane solubility and live oil density calculations is presented in Appendix C.

\subsection{Experimental Error}

Table 3.4 shows the instrument's range of operation and their accuracy used in this study. 
Table 3.4 Instrument's range and accuracy

\begin{tabular}{|c|c|c|}
\hline Instrument & Range & Accuracy \\
\hline Load cell & -54 to $93^{\circ} \mathrm{C} 0-3500$ (g) & $\pm 0.02 \%$ of full scale \\
\hline Pressure transducer & -54 to $121^{\circ} \mathrm{C} \quad 0-200$ (psig) & $\pm 0.1 \%$ of full scale \\
\hline Resistance Temp. Detectors & $0-230^{\circ} \mathrm{C} \quad 0-2500 \mathrm{psig}$ & $\pm 0.3^{\circ} \mathrm{C}$ \\
\hline Differential pressure transducer & $0-30$ (psid) & $\pm 0.5 \%$ of full scale \\
\hline Thermocouple (Type T) & Up to $260^{\circ} \mathrm{C}$ & $\pm 0.5^{\circ} \mathrm{C}$ \\
\hline Flow meter & -10 to $+50{ }^{\circ} \mathrm{C} 125 \mathrm{psig}(\mathrm{max})$ & $\pm 0.8 \%$ of reading \\
\hline Temperature controller & -10 to $50^{\circ} \mathrm{C}$ & $\pm 0.5^{\circ} \mathrm{C}$ \\
\hline
\end{tabular}

\subsection{Residual Oil Saturation}

Residual oil saturation is the measurement of how much oil in place is left compared to the pore volume of a system. The porous medium used in this study were initially fully saturated, then the volume of oil in place was equal to the pore volume of the system. The mathematical expression for residual oil is:

residual oil saturation $=\frac{\text { residual oil mass }}{\text { mass of initial oil in place }}$

Several samples were taken from different sections of the physical model as shown in Figure 3.7. Each sample was removed from the model and weighed. Then, the sample was rinsed repeatedly with toluene until the beads became clean. Acetone is used for the final rinse. The glass beads were then dried in a convection oven for 30 minutes and then reweighed again.

The residual oil saturation was calculated as:

residual oil saturation $=\frac{\text { residual oil mass }}{\text { mass of saturated oil }}=\frac{\text { residual oil mass }}{\mathrm{V}_{\text {bulk }} \varphi \rho_{\text {bitumen }}}$

$\mathrm{V}_{\text {bulk }}=\frac{\text { mass of beads }}{(1-\varphi) \rho_{\text {beads }}}=\frac{V_{\text {pore }}}{\varphi}$ 
where $\mathrm{V}_{\text {bulk }}$ is the bulk volume of the sample, $\rho_{\text {bitumen }}$ is the bitumen density, $\rho_{\text {beads }}$ is the glass beads density, $\mathrm{V}_{\text {pore }}$ is the pore volume.

Averaged residual oil saturation obtained for all experiments varied between 4 to $10 \%(\mathrm{PV})$.

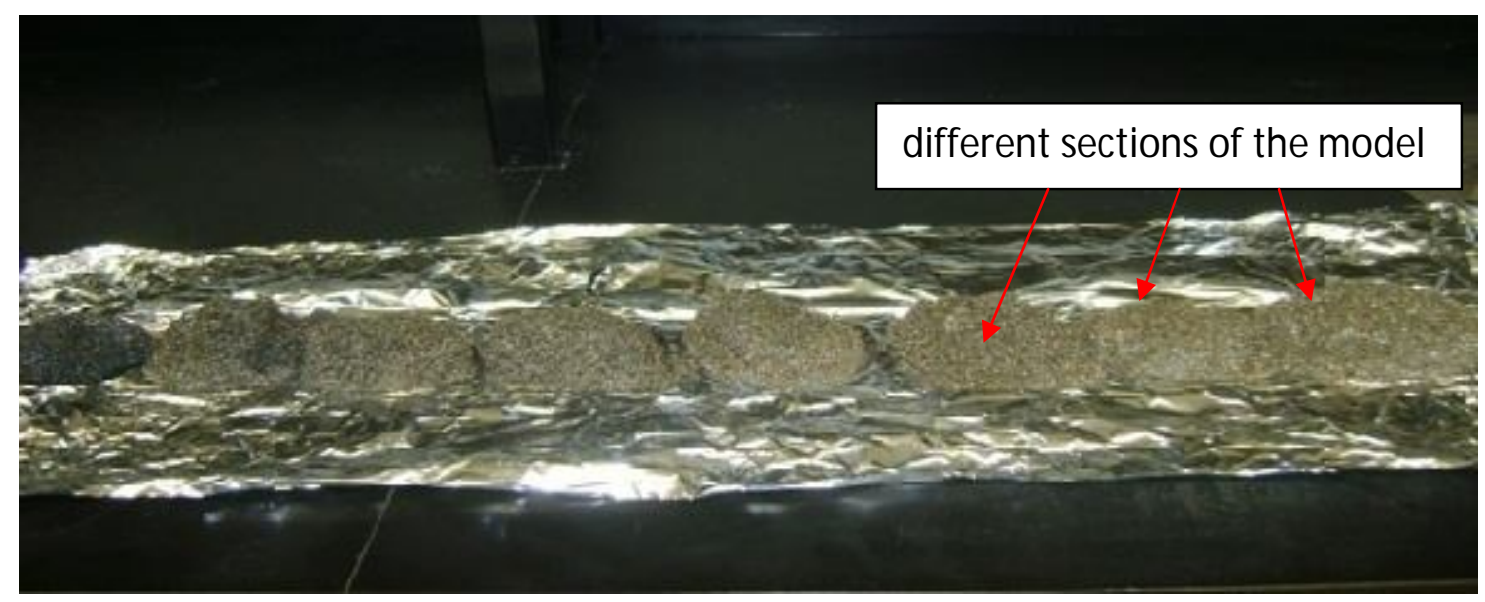

Figure 3.7 Samples from different sections of the physical model 


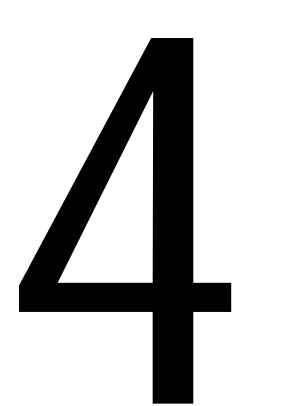

\section{Theory and Computations}

In this chapter, the determination of the solvent concentration-dependent dispersion coefficient is presented as an optimal problem. The fundamental in optimal control theory is to determine the policy that will minimize (maximize) some performance criterion, subject to the constraints, which are imposed by the physical nature of the problem. The principles of the theory are based on the classical calculus of variations. The fundamental theorem of the calculus of variations is applied to problems with unconstrained states and controls. The variational approach generates highly non linear differential equations that require the use of numerical techniques for solution.

The formulation of an optimal control problem requires a mathematical model characterizing the process to be optimized and the physical constraints. A mass transfer model of vapor extraction of heavy oil using a solvent is developed. The model has an undetermined concentrationdependent dispersion function. Incorporating this function in the mass transfer model, the modelcalculated mass of oil produced should be equal to its measured value obtained from the experiments. The conditions that are necessary to determine the optimal dispersion function are derived. Finally, a numerical algorithm is developed to compute the solvent dispersion as a function of its concentration in heavy oil.

\subsection{Mass Transfer Model of Vapex}

A mathematical model is developed here to describe the mass transfer process based on the vapor extraction experiments. The mass transfer model implements the continuity equation for 
solvent that diffuses into bitumen. The model assumptions along with their justification are as follows:

1. Vapex process is carried out at constant temperature and pressure.

-The temperature of the system is maintained uniform and constant by controlling the water bath temperature surrounding the pressure vessel. The relative deviations for the system temperature and the water bath temperature are found to lie between $\pm 2 \%$ as shown in Appendix E, which indicates a good temperature control. The pressure of the system is maintained constant by a pressure regulator installed on the propane cylinder.

2. Solvent diffusion is along the radial direction only.

-Diffusion occurs in the radial direction over a large surface area along the circumference of the cylindrical model. In contrast, diffusion in the vertical direction occurs over a much smaller area at the top of the cylindrical model. Therefore, diffusion in the vertical direction is neglected.

3. The velocity of the live oil along the vertical direction is governed by Darcy's law in a porous medium.

-The live oil flow is a creeping flow since the Reynold's numbers ( Re ) calculated for the live oil flow are less than unity.

4. The porous medium has uniform porosity and permeability.

-The particles size distributions for the glass beads used in this work are uniform as discussed in Section 3.4. Moreover, all the beads are uniformly sized spheres and each physical model composes of one type of glass beads saturated with heavy oil. Thus, each model has a uniform permeability and no heterogeneities exist.

5. The density of the live oil is assumed constant.

-The small variations in the live oil density measured experimentally as shown in Table 5.2, allows us to assume constant live oil density. Moreover, a sensitivity study conducted (see Section 5.2.6) shows that the effect of the density variation on the dispersion values is insignificant.

6. The heavy oil is non-volatile.

-The heavy oil is heated for at least 30 minutes at $70^{\circ} \mathrm{C}$ before it is saturated with the glass beads. Thus, no volatile components exist in the heavy oil. 
The unsteady state mass balance for solvent propane over a differential element of the medium (see Figure 4.1) is given by

$\frac{d}{d t}(V \varphi \rho \omega)=(v A \rho \omega)_{z+\Delta z}-(v A \rho \omega)_{z}+\left(J_{\mathrm{f}} S \varphi\right)_{r+\Delta r}-\left(J_{\mathrm{f}} S \varphi\right)_{r}$

where $V=2 \pi r \Delta r \Delta z$ the volume of the element, $A=2 \pi r \Delta r$ is the area transverse to the live oil velocity $v$ in the vertical direction, and $S=2 \pi r \Delta \mathrm{z}$ is the area transverse to the dispersive flux $J_{\mathrm{f}}$ in the radial direction.

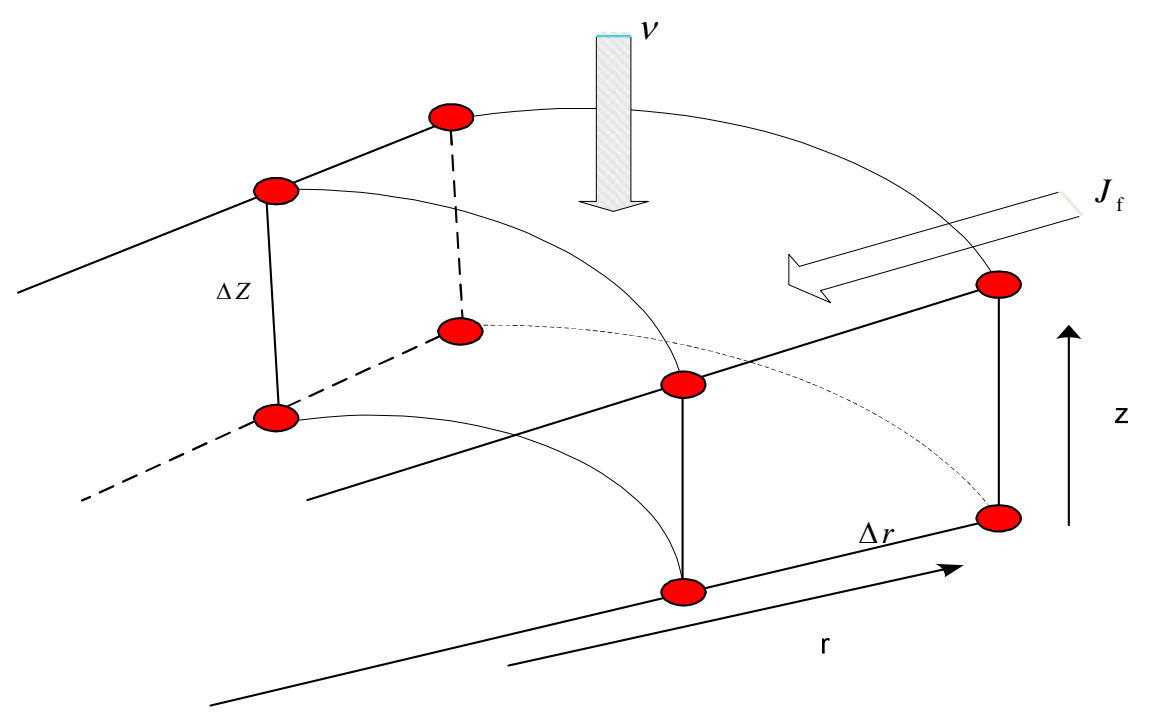

Figure 4.1 Differential element of the physical model.

The above mathematical model developed in this work (Equation (4.1)) has the following modifications in comparison with previous work published in the literature (El-Haj et al., 2009; Imran, 2008):

-In Equation (4.1), the dispersive flux in the radial direction is multiplied by the porosity of the porous medium since flow takes place only through part of the transverse area.

-The convectional (mechanical) dispersion flux along the vertical direction is assumed insignificant in comparison with the convective flux. When the convectional (mechanical) flux term of the solvent in the vertical direction is incorporated in the mathematical model, the 
calculated mechanical flux is found to be of the order $10^{-10}$. In contrast, the convective flux is found to be of the order $10^{-6}$.

-The dispersion function, $D(\omega)$, is an undetermined concentration-dependent dispersion coefficient that is not constrained with any functional form.

The radial flux can be written as

$$
J_{\mathrm{f}}=-D \rho \frac{d \omega}{d r}
$$

where $D$ is the undetermined concentration-dependent dispersion coefficient of propane in the porous medium. Taking the limits of $\Delta r$ and $\Delta z$ to zero, the above equations yield the following mass transfer model (Abukhalifeh et al., 2009):

$$
\frac{\partial \omega}{\partial t}=\frac{1}{r}\left[D\left(\frac{\partial \omega}{\partial r}\right)+r D\left(\frac{\partial^{2} \omega}{\partial r^{2}}\right)+r\left(\frac{\partial D}{\partial \omega}\right)\left(\frac{\partial \omega}{\partial r}\right)^{2}\right]-\frac{1}{\varphi}\left[v\left(\frac{\partial \omega}{\partial z}\right)+\omega\left(\frac{\partial v}{\partial \omega}\right)\left(\frac{\partial \omega}{\partial z}\right)\right]
$$

where $\omega(t, r, z)$ is the mass fraction of solvent in bitumen, which is a function of time, radius, and height of the porous medium; $v$ is the Darcy velocity of the live oil and is given by

$$
v=\frac{K K_{r} \rho g \cos \theta}{\mu}
$$

where $K_{\mathrm{r}}$ is relative permeability of the medium, $K$ is its permeability, $\rho$ is the density of live oil, $g$ is gravity, $\mu$ is the live oil viscosity, and $\theta$ is the inclination angel of the physical model with the vertical axis. Since the porous medium is saturated only with heavy oil, the effective permeability equals the absolute permeability, and then the relative permeability is equal to unity. 
Experimental live oil viscosity and propane mass fraction in heavy oil data were best fitted to obtain the live oil viscosity concentration-dependent model. The applicable empirical correlation for the propane-heavy oil system for this experiment at the operating temperature and pressure is:

$$
\mu=\mu_{0} \omega^{-2} \quad 0.28 \leq \omega \leq 0.45
$$

Similar relationships for the viscosity models of various solvent-heavy oil systems were obtained by Jin (1999) and Ramakrishnan (2003).

Substitution the above expression for $\mu$, Equation (4.4) is simplified to

$v=\frac{K K_{r} \rho g \cos \theta}{\mu_{o}} \omega^{2}=\alpha \omega^{2}$

where

$\alpha=\frac{K K_{r} \rho g \cos \theta}{\mu_{o}}$

The live oil drainage with time reduces the height of the bitumen, $Z(t, r)$, in the packed medium. The change in the height with time at any radial location is given by

$\frac{\partial Z}{\partial t}=-v(t, r, 0)$

where $v(t, r, 0)$ is Darcy velocity at the bottom of the model at a given $r$. Initially there is no gas inside the packing and no production of the live oil. The initial height of the bitumen sample is $Z_{0}$. The packing surface has the solvent concentration equal to its interface concentration under prevailing temperature and pressure. Thus, the initial conditions at $t=0$ are as follows: 


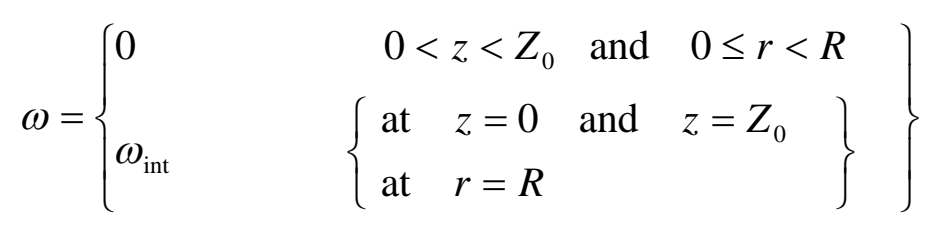

At all times, the entire exposed circumference and the top face of the cylinder has the solvent concentration equals to its interface concentration, and there is no mass transfer at the bottom of the cylinder. The solvent-heavy oil interface at the top of the physical model moves down and the height of the bitumen, $Z(t, r)$, decreases with time due to live oil drainage. Thus, we have a moving boundary problem, which is described by Equation (4.8).

The solvent concentration at the moving interface is equal to the solvent interface concentration at all times. Consequently, the boundary conditions at $t>0$ are given by

$$
\omega=\omega_{\text {int }} \begin{cases}0 \leq z \leq Z, & r=R \\ 0 \leq r<R & Z=Z\end{cases}
$$

Because of symmetry, at all times:

$$
\frac{\partial \omega}{\partial r}=0 \quad \text { at } r=0 \quad \text { for all } \quad 0 \leq z \leq Z
$$

Substitution the above expression for $v$, Equation (4.3) yields

$$
\frac{\partial \omega}{\partial t}=D\left[\frac{1}{r}\left(\frac{\partial \omega}{\partial r}\right)+\left(\frac{\partial^{2} \omega}{\partial r^{2}}\right)\right]+\left[\left(\frac{\partial D}{\partial \omega}\right)\left(\frac{\partial \omega}{\partial r}\right)^{2}\right]-\frac{3}{\varphi} \frac{K_{\mathrm{r}} K \rho g \cos \theta}{\mu_{0}} \omega^{2}\left(\frac{\partial \omega}{\partial z}\right)
$$

Solution of Equation (4.12) furnishes the solvent mass fraction in bitumen, i.e., $\omega(t, r, z)$. The cumulative mass of produced live oil at any time is given by 


$$
m_{c}=\varphi \int_{0}^{R} 2 \pi \rho r\left(Z_{o}-Z\right) \mathrm{d} r
$$

\subsection{The Optimal Control Problem}

Now, an optimal control problem will be formulated to determine the desired dispersion function that would minimize the difference between the model-predicted and the experimental measured values of the cumulative live oil produced. The conditions that are necessary to obtain the desired dispersion function will be fundamentally derived in the upcoming subsections, which needs some familiarity with calculus of variations (Courant and Hilbert 1953; Kirk 1970).

\subsubsection{Objective Functional}

It is desired to find the optimal dispersion function, $D(\omega)$, such that the difference between the model-calculated and experimental measured cumulative live oil produced is minimum. Mathematically, the objective functional can be written as

$$
I_{\min }=\int_{0}^{T}\left[m_{\mathrm{c}}(t)-m_{\mathrm{e}}(t)\right]^{2} \mathrm{~d} t
$$

and it is calculated as

$$
I=\sum_{t=1}^{T}\left[m_{c}(t)-m_{e}(t)\right]^{2} \Delta t
$$

where $I$ is the objective functional that needs to be minimized using the control function $D=D[\omega(t, r, z)] ; m_{e}(t)$ is the experimental cumulative mass of the live oil produced at any time $t$, and $m_{c}(t)$ is the model cumulative predicted mass of the live oil produced at any time $t$ and 
$T$ is the total operating time. The calculated mass $m_{\mathrm{c}}(t)$ is given by Equation (4.13). Now, Equation (4.14) can be written as

$$
I=\int_{0}^{T}\left[m_{c}(t)-m_{e}(t)\right]^{2} \mathrm{~d} t=\int_{0}^{T}\left[\varphi \int_{0}^{R} 2 \pi \rho r\left(Z_{0}-Z\right) \mathrm{d} r-m_{e}(t)\right]^{2} \mathrm{~d} t
$$

subject to Equation (4.3), which in turn can be written as

$$
\frac{\partial \omega}{\partial t}-f_{1}=G(t, r, z)=0
$$

where

$$
f_{1}=-\frac{3 \alpha \omega^{2}}{\varphi}\left(\frac{\partial \omega}{\partial z}\right)+\frac{D}{r}\left(\frac{\partial \omega}{\partial r}\right)+D\left(\frac{\partial^{2} \omega}{\partial r^{2}}\right)+\left(\frac{\partial D}{\partial \omega}\right)\left(\frac{\partial \omega}{\partial r}\right)^{2}
$$

and subject to Equation (4.8), which can be written as

$$
\frac{\partial Z}{\partial t}-f_{2}=F(t, r)=0
$$

where

$$
f_{2}=-v(t, r, 0)=-\alpha[\omega(t, r, 0)]^{2}
$$


Equations (4.17) and (4.19) are the constraints for Equation (4.14), $D(\omega)$ is the control functional. As an alternative approach, Lagrange multipliers (sometimes called adjoint or costate variables) are used. Lagrange multipliers, or costate variables, are used to ensure that constraints given by the equations of the simulation model are satisfied. The adjoint variables $\lambda(t, r, z)$ and $\gamma(t, r)$ are introduced to form the augmented functional by adjoining the constraining relations to $I$. Note that for any $\lambda$ and $\gamma, J=I$ if the constraints are satisfied. Introducing the two adjoint variables into Equation (4.14) yields the following unconstrained objective functional:

$J=I+\int_{0}^{T} \int_{0}^{R} \int_{0}^{Z} \lambda(t, r, z) G(t, r, z) \mathrm{d} z \mathrm{~d} r \mathrm{~d} t+\int_{0}^{T} \int_{0}^{R} \gamma(t, r) F(t, r) \mathrm{d} r \mathrm{~d} t$

Substituting for $G(t, r, z)$ and $F(t, r)$ in the above equation yields

$$
\begin{aligned}
& J=\int_{0}^{T}\left(m_{c}(t)-m_{e}(t)\right)^{2} \mathrm{~d} t \\
& +\int_{0}^{T} \int_{0}^{R} \int_{0}^{Z} \lambda\left(\frac{\partial \omega}{\partial t}+\frac{3 \alpha \omega^{2}}{\varphi}\left(\frac{\partial \omega}{\partial z}\right)-\frac{D}{r}\left(\frac{\partial \omega}{\partial r}\right)-D\left(\frac{\partial^{2} \omega}{\partial r^{2}}\right)-\frac{\partial D}{\partial \omega}\left(\frac{\partial \omega}{\partial r}\right)^{2}\right) \mathrm{d} z \mathrm{~d} r \mathrm{~d} t \\
& +\int_{0}^{T} \int_{0}^{R} \gamma\left(\frac{\partial Z}{\partial t}+\alpha \omega(t, r, 0)^{2}\right) \mathrm{d} r \mathrm{~d} t
\end{aligned}
$$

The minimization of $J$ is now equivalent to the minimization of $I$. The variational derivative of $J$ with respect to the optimization variable $D$ will provide the conditions necessary for the minimum of $J$.

\subsubsection{Necessary Conditions}

In this section, we derive the necessary conditions for the minimum of $J$. Consider the variation of $J$ as follows: 
$\delta J=\delta I+\int_{0}^{T} \int_{0}^{R} \int_{0}^{Z} \lambda(t, r, z) \delta G(t, r, z) \mathrm{d} z \mathrm{~d} r \mathrm{~d} t+\int_{0}^{T} \int_{0}^{R} \gamma(t, r) \delta F(t, r) \mathrm{d} r \mathrm{~d} t$

where

$$
\begin{aligned}
\delta I & =\int_{0}^{T} 2\left[m_{\mathrm{c}}(t)-m_{\mathrm{e}}(t)\right] \int_{0}^{R} 2 \varphi \pi \rho r(-1) \delta \mathrm{Zd} r \mathrm{~d} t \\
& =-\int_{0}^{T} \int_{0}^{R} 4 \phi \pi \rho r\left[m_{\mathrm{c}}(t)-m_{\mathrm{e}}(t)\right] \delta \mathrm{Zd} r \mathrm{~d} t
\end{aligned}
$$

$\delta F=\frac{\partial(\delta Z)}{\partial t}-\delta f_{2}$

Substitution of Equations (4.24), (4.25), and (4.26) into Equation (4.23) yields

$$
\begin{aligned}
& \delta J=-\int_{0}^{T} \int_{0}^{R} 4 \varphi \pi \rho r\left[m_{\mathrm{c}}(t)-m_{\mathrm{e}}(t)\right] \delta \mathrm{Z} \mathrm{d} t \mathrm{~d} r \\
& +\left[\begin{array}{l}
\int_{0}^{T} \int_{0}^{R} \int_{0}^{Z} \lambda \frac{\partial(\delta \omega)}{\partial t} \mathrm{~d} z \mathrm{~d} r \mathrm{~d} t-\int_{0}^{T} \int_{0}^{R} \int_{0}^{Z} \lambda\left(\frac{\partial f_{1}}{\partial \omega}\right) \delta \omega \mathrm{d} z \mathrm{~d} r \mathrm{~d} t-\int_{0}^{T} \int_{0}^{R} \int_{0}^{Z} \lambda\left(\frac{\partial f_{1}}{\partial \omega_{z}}\right) \delta \omega_{\mathrm{z}} \mathrm{d} z \mathrm{~d} r \mathrm{~d} t \\
\left.-\int_{0}^{T} \int_{0}^{R} \int_{0}^{Z} \lambda\left(\frac{\partial f_{1}}{\partial \omega_{\mathrm{r}}}\right) \delta \omega_{\mathrm{r}} \mathrm{d} z \mathrm{~d} r \mathrm{~d} t-\int_{0}^{T} \int_{0}^{R} \int_{0}^{Z} \lambda\left(\frac{\partial f_{1}}{\partial \omega_{\mathrm{rr}}}\right) \delta \omega_{\mathrm{rr}} \mathrm{d} z \mathrm{~d} r \mathrm{~d} t-\int_{0}^{T} \int_{0}^{R} \int_{0}^{Z} \lambda\left(\frac{\partial f_{1}}{\partial D}\right) \delta D \mathrm{~d} z \mathrm{~d} r \mathrm{~d} t\right] \\
+\left[\int_{0}^{T} \int_{0}^{R} \gamma \frac{\partial(\delta Z)}{\partial t} \mathrm{~d} r \mathrm{~d} t-\int_{0}^{T} \int_{0}^{R} \gamma \delta f_{2} \mathrm{~d} r \mathrm{~d} t\right]
\end{array}\right.
\end{aligned}
$$


Integration by parts of the first integral of the second term of the above equation yields

$$
\int_{0}^{T} \int_{0}^{R} \int_{0}^{Z} \lambda \frac{\partial(\delta \omega)}{\partial t} \mathrm{~d} z \mathrm{~d} r \mathrm{~d} t=\int_{0}^{R} \int_{0}^{Z}[\lambda \delta \omega]_{0}^{T} \mathrm{~d} z \mathrm{~d} r-\int_{0}^{R} \int_{0}^{Z} \int_{0}^{T}\left(\frac{\partial \lambda}{\partial t}\right) \delta \omega \mathrm{d} t \mathrm{~d} z \mathrm{~d} r
$$

The first integral on right hand side in Equation (4.28) is eliminated based on the nature of the process as follows: Because the solvent mass fraction is known at $t=0$, its variation is ruled out, i.e.

$\delta \omega(0, r, z)=0$

The final mass fraction of solvent in bitumen is not specified. Thus, the variation due to the mass fraction is eliminated if its multiplicative term is forced to zero, i.e.

$\lambda(T, r, z)=0$

Substitution of Equation (4.29) and Equation (4.30) into Equation (4.28) results in

$$
\int_{0}^{T} \int_{0}^{R} \int_{0}^{Z} \lambda \frac{\partial(\delta \omega)}{\partial t} \mathrm{~d} z \mathrm{~d} r \mathrm{~d} t=-\int_{0}^{T} \int_{0}^{R} \int_{0}^{Z}\left(\frac{\partial \lambda}{\partial t}\right) \delta \omega \mathrm{d} z \mathrm{~d} r \mathrm{~d} t
$$

Integration by parts of the third integral of the second term in Equation (4.27) yields

$$
\int_{0}^{T} \int_{0}^{R} \int_{0}^{Z}-\lambda\left(\frac{\partial f_{1}}{\partial \omega_{\mathrm{z}}}\right) \delta \omega_{\mathrm{z}} \mathrm{d} z \mathrm{~d} r \mathrm{~d} t=-\int_{0}^{T} \int_{0}^{R}\left\{\left[\lambda\left(\frac{\partial f_{1}}{\partial \omega_{\mathrm{z}}}\right) \delta \omega_{0}^{Z}-\int_{0}^{Z} \frac{\partial}{\partial z}\left(\lambda \frac{\partial f_{1}}{\partial \omega_{\mathrm{z}}}\right) \delta \omega d z\right\} \mathrm{d} r \mathrm{~d} t\right.
$$


Since the solvent mass fraction in bitumen is specified for all $r$ and $t$, the variation $\delta \omega(t, r, Z)=0$ Hence,

$$
\int_{0}^{T} \int_{0}^{R} \int_{0}^{Z}-\lambda\left(\frac{\partial f_{1}}{\partial \omega_{\mathrm{z}}}\right) \delta \omega_{\mathrm{z}} \mathrm{d} z \mathrm{~d} r \mathrm{~d} t=\int_{0}^{T} \int_{0}^{R}\left[\lambda\left(\frac{\partial f_{1}}{\partial \omega_{\mathrm{z}}}\right) \delta \omega(t, r, 0)+\int_{0}^{T} \int_{0}^{R} \int_{0}^{Z} \frac{\partial}{\partial z}\left(\lambda \frac{\partial f_{1}}{\partial \omega_{\mathrm{z}}}\right) \delta \omega\right] \mathrm{d} z \mathrm{~d} r \mathrm{~d} t
$$

Integration by parts of the fourth integral of the second term of Equation (4.27) yields

$$
\int_{0}^{T} \int_{0}^{R} \int_{0}^{Z}-\lambda\left(\frac{\partial f_{1}}{\partial \omega_{\mathrm{r}}}\right) \delta \omega_{\mathrm{r}} \mathrm{d} z \mathrm{~d} r \mathrm{~d} t=-\int_{0}^{\mathrm{T}} \int_{0}^{\mathrm{Z}}\left\{\left[\lambda \frac{\partial f_{1}}{\partial \omega_{\mathrm{r}}} \delta \omega\right]_{0}^{R}-\int_{0}^{R} \frac{\partial}{\partial r}\left(\lambda \frac{\partial f_{1}}{\partial \omega_{\mathrm{r}}}\right) \delta \omega \mathrm{d} r\right\} \mathrm{d} z \mathrm{~d} t
$$

Since the solvent mass fraction in bitumen, $\omega(t, R, z)$, is known for all $z$ and $t$, the variation is zero. The mass fraction of solvent in bitumen, $\omega(t, 0, z)$, is not specified. Variation due to $\omega(t, 0, z)$ is eliminated if its multiplicative term is forced to zero, i.e.

$\lambda(t, 0, z)=0$

The above equation leads to

$$
\int_{0}^{T} \int_{0}^{R} \int_{0}^{Z}-\lambda\left(\frac{\partial f_{1}}{\partial \omega_{\mathrm{r}}}\right) \delta \omega_{\mathrm{r}} \mathrm{d} z \mathrm{~d} r \mathrm{~d} t=\int_{0}^{T} \int_{0}^{Z} \int_{0}^{R} \frac{\partial}{\partial r}\left[\lambda \frac{\partial f_{1}}{\partial \omega_{\mathrm{r}}}\right] \delta \omega \mathrm{d} z \mathrm{~d} r \mathrm{~d} t
$$

Integration by parts of the fifth integral of the second term of Equation (4.27) yields 


$$
\begin{aligned}
& \int_{0}^{T} \int_{0}^{R} \int_{0}^{Z}-\lambda\left(\frac{\partial f_{1}}{\partial \omega_{\mathrm{rr}}}\right) \delta \omega_{\mathrm{rr}} \mathrm{d} z \mathrm{~d} r \mathrm{~d} t= \\
& -\int_{0}^{T} \int_{0}^{Z}\left\{\left[\lambda \frac{\partial f_{1}}{\partial \omega_{\mathrm{rr}}} \delta \omega_{\mathrm{r}}\right]_{0}^{R}-\left(\left[\frac{\partial}{\partial r}\left(\lambda \frac{\partial f_{1}}{\partial \omega_{\mathrm{rr}}}\right) \delta \omega\right]_{0}^{R}-\int_{0}^{R} \frac{\partial^{2}}{\partial r^{2}}\left(\lambda \frac{\partial f_{1}}{\partial \omega_{\mathrm{rr}}}\right) \delta \omega \mathrm{d} r\right)\right\} \mathrm{d} z \mathrm{~d} t
\end{aligned}
$$

Application of Equation (4.35) eliminates the second term on right hand side of Equation (4.37). To eliminate the first term on right hand side of Equation (4.37), the multiplicative term is forced to zero, i.e.

$\lambda(t, R, z)=0$

The above conditions reduce Equation (4.37) to

$$
\int_{0}^{T} \int_{0}^{R} \int_{0}^{Z}-\lambda\left(\frac{\partial f_{1}}{\partial \omega_{\mathrm{rr}}}\right) \delta \omega_{\mathrm{rr}} \mathrm{d} z \mathrm{~d} r \mathrm{~d} t=-\int_{0}^{T} \int_{0}^{Z} \int_{0}^{R} \frac{\partial^{2}}{\partial r^{2}}\left[\lambda \frac{\partial f_{1}}{\partial \omega_{\mathrm{rr}}}\right] \delta \omega \mathrm{d} z \mathrm{~d} r \mathrm{~d} t
$$

Integration by parts of the integral of the third term of Equation (4.27) yields

$$
\int_{0}^{T} \int_{0}^{R} \gamma\left[\frac{\partial(\delta Z)}{\partial t}\right] \mathrm{d} r \mathrm{~d} t=\int_{0}^{R}\left[[\gamma \delta Z]_{0}^{T}-\int_{0}^{T} \frac{\partial \gamma}{\partial t} \delta Z \mathrm{~d} t\right] \mathrm{d} r
$$

The first integral on right hand side in Equation (4.40) is eliminated as follows. The initial height of bitumen, $Z(0, r)$, is known, then the variation of $Z(0, r)$ is ruled out, i.e.

$$
\delta Z(0, r)=0
$$


The final height of bitumen, $Z(T, r)$, is not specified. Variation due to the final height is eliminated if its multiplicative term is forced to zero, i.e.

$\gamma(T, r)=0$

Substitution of Equation (4.41) and Equation (4.42) in Equation (4.40) results in

$\int_{0}^{T} \int_{0}^{R} \gamma\left(\frac{\partial(\delta \mathrm{Z})}{\partial t}\right) \mathrm{d} r \mathrm{~d} t=-\int_{0}^{R} \int_{0}^{T} \frac{\partial \gamma}{\partial t} \delta \mathrm{Z} \mathrm{d} r \mathrm{~d} t$

The second integral of the third term of Equation (4.27) yields

$-\int_{0}^{T} \int_{0}^{R} \gamma \delta f_{2} \mathrm{~d} r \mathrm{~d} t=-\int_{0}^{T} \int_{0}^{R} \gamma\left(\frac{\partial f_{2}}{\partial \omega(t, r, 0)}\right) \delta \omega(t, r, 0) \mathrm{d} r \mathrm{~d} t$

Finally, substitution of the above expressions into Equation (4.27) results in

$$
\begin{aligned}
& \delta J=\int_{0}^{T} \int_{0}^{R}\left[-4 \varphi \pi \rho r\left[m_{\mathrm{c}}(t)-m_{\mathrm{e}}(t)\right]-\frac{\partial \gamma}{\partial t}\right] \delta Z \mathrm{~d} r \mathrm{~d} t \\
& +\int_{0}^{T} \int_{0}^{R} \int_{0}^{Z}\left[-\frac{\partial \lambda}{\partial t}-\lambda\left(\frac{\partial f_{1}}{\partial \omega}\right)+\frac{\partial}{\partial z}\left(\lambda \frac{\partial f_{1}}{\partial \omega_{\mathrm{z}}}\right)+\frac{\partial}{\partial r}\left(\lambda \frac{\partial f_{1}}{\partial \omega_{\mathrm{r}}}\right)-\frac{\partial^{2}}{\partial r^{2}}\left(\lambda \frac{\partial f_{1}}{\partial \omega_{\mathrm{rr}}}\right)\right] \delta \omega \mathrm{d} z \mathrm{~d} r \mathrm{~d} t \\
& +\int_{0}^{T} \int_{0}^{R}\left[\lambda\left(\frac{\partial f_{1}}{\partial \omega_{z}(t, r, 0)}\right)-\gamma\left(\frac{\partial f_{2}}{\partial \omega(t, r, 0)}\right)\right] \delta \omega(t, r, 0) \mathrm{d} r \mathrm{~d} t \\
& +\int_{0}^{T} \int_{0}^{R} \int_{0}^{Z}\left[-\lambda\left(\frac{\partial f_{1}}{\partial D}\right)\right] \delta D \mathrm{~d} z \mathrm{~d} r \mathrm{~d} t
\end{aligned}
$$


The fundamental theorem of the calculus of variations is now applied to determine the necessary conditions for the function $D(\omega)$ to provide a minimum for the objective functional. The fundamental theorem states that the variation of the functional must vanish on an extremal, i.e. at the minimum, $\delta J$ given by Equation (4.45) should be zero. That is only possible when the variational derivative of $J$ with respect to $D$ is

$\frac{\partial J}{\partial D}=-\lambda \frac{\partial f_{1}}{\partial D}=0$

subject to the following adjoint equations:

$$
\begin{aligned}
& \frac{\partial \lambda}{\partial t}=-\lambda\left(\frac{\partial f_{1}}{\partial \omega}\right)+\frac{\partial}{\partial z}\left(\lambda \frac{\partial f_{1}}{\partial \omega_{\mathrm{z}}}\right)+\frac{\partial}{\partial r}\left(\lambda \frac{\partial f_{1}}{\partial \omega_{\mathrm{r}}}\right)-\frac{\partial^{2}}{\partial r^{2}}\left(\lambda \frac{\partial f_{1}}{\partial \omega_{\mathrm{rr}}}\right) \\
& \frac{\partial \gamma}{\partial t}=-4 \varphi \pi \rho\left[m_{\mathrm{c}}(t)-m_{\mathrm{e}}(t)\right] r \\
& {\left[\lambda \frac{\partial f_{1}}{\partial \omega_{\mathrm{z}}}\right]_{z=0}-\left[\gamma \frac{\partial f_{2}}{\partial \omega}\right]_{z=0}=0}
\end{aligned}
$$

Thus, Equation (4.46) is the necessary condition for the minimization of $J$ when the continuity equation, as well as the adjoint equations [Equations (4.47)-(4.49)] are satisfied.

\subsubsection{Adjoint Equations}

Using Equation (4.18), (4.20), (4.47) and (4.49) we obtain

$$
\frac{\partial \lambda}{\partial t}=\frac{-3 \alpha \omega^{2}}{\varphi}\left(\frac{\partial \lambda}{\partial z}\right)-\frac{\lambda D}{r^{2}}+\frac{D}{r}\left(\frac{\partial \lambda}{\partial r}\right)-D\left(\frac{\partial^{2} \lambda}{\partial r^{2}}\right)
$$


$\frac{-3 \lambda \alpha[\omega(t, r, 0)]^{2}}{\varphi}=-2 \gamma \alpha[\omega(t, r, 0)]$

The boundary conditions for Equation (4.50) are:

$\lambda(T, r, z)=0$

$\lambda(t, 0, z)=\lambda(t, R, z)=0$

The boundary condition for Equation (4.48) is:

$\gamma(T, r)=0$

\subsection{Solution of the Optimal Problem}

Restating the optimal problem, an optimal value of $D(\omega)$ needs to be calculated such that the augmented objective functional $J$ defined by Equation (4.22) is minimum. As stated earlier, the above is equivalent to the minimization of the objective functional $I$ defined by Equation (4.16).

Variational techniques were used to derive necessary conditions for $D(\omega)$ to be an extremal of the functional $J$. The variational approach leads to non-linear partial differential equations that cannot be solved analytically to obtain the optimal $D(\omega)$. Therefore, the problem is solved numerically. An iterative numerical technique was used to determine the optimal dispersion function. The computational procedure is based on adjusting estimates of the dispersion function to improve the value of the objective functional. For the dispersion function to be optimal, the necessary condition given by Equation (4.46) must be satisfied along with Equation (4.3), Equation (4.8) and the adjoint equations. If the dispersion function is not optimal, then a correction in the gradient direction ensures an improvement in the objective functional. 


\subsubsection{The Gradient Improvement Method}

A gradient method was adapted for iterative solution of this optimal control problem. The greatest local rate of decrease in the value of a function with respect to its variables is in the direction opposite to its gradient. This direction is called the steepest descent direction. The negative of the variational derivative for $J$ gives its steepest descent direction at $r$ and $z$ and time $t$. Equation (4.46) is used to set the search direction to find the minimum of $J$.

Quasi-Newton methods (or called variable metric methods) are the most favored of the methods that use gradient of a function. These methods compute a search direction utilizing first order gradient information. Newton's methods make use of the second order information (Hessian matrix) of a function. Hessian matrix is calculated numerically, which involve a large amount of computations. Quasi-Newton methods avoid the calculation of exact Hessian by generating approximate Hessian matrix using an appropriate updating technique. A large number of Hessian updating methods have been developed. However, the formula derived by Broyden 1970, Fletcher 1970, Goldfarb 1970, and Shanno 1970 (BFGS) is thought to be the most effective method. BFGS method is a numerical algorithm to find optimal solution for unconstrained nonlinear problems. It utilizes first order gradient information to generate approximate Hessian matrix.

\subsubsection{Implementation of the Improvement Method}

The search direction was set by the variational derivative $J_{D}$ given by

$$
J_{\mathrm{D}}[\omega(t, r, z)]=\frac{\partial J}{\partial D}=-\frac{\lambda}{r} \frac{\partial \omega}{\partial r}-\lambda \frac{\partial^{2} \omega}{\partial r^{2}}
$$

The gradient correction $J_{D}$ was expressed in a finite-difference form along $r$ direction to be utilized in the simulation. Differential changes are averaged at any concentration, which has multiple existences in the height domain. The values of differential changes are time-averaged before their usage for the gradient correction in $D(\omega)$ by the BFGS method. 
In addition, the gradient correction $J_{\mathrm{D}}$ was scaled to the magnitude of current dispersion values as follows:

$$
J_{\mathrm{DS}}(k)=\varepsilon^{2} \frac{J_{\mathrm{D}}(k)}{\left\|J_{\mathrm{D}}\right\|}\|D\| \quad \text { for } \quad k=1,2, \ldots \ldots, n
$$

where $\varepsilon^{2}$ is a small adjustable parameter, $J_{\mathrm{DS}}(k)$ is the scaled gradient correction at a specified gas mass fraction of the dispersion function, and $n$ is the number of specified gas mass fractions of the dispersion function. Using the scaled gradient correction, the iterative improvement in the value of $D(\omega)$ was given by

$$
D_{i+1}=D_{i}-\beta_{i} J_{\mathrm{DS}, i}
$$

where $\beta_{i}$ is the optimal step length along the search direction in the $i$ th iteration. The optimal step length is found by a line minimization method (Press et al., 2002).

\subsubsection{Integration of Continuity and Adjoint Equations}

To implement the numerical solution, Equations (4.17), (4.19), (4.48) and (4.50) were expressed in a finite-difference form along $r$ and $z$ directions. The cylindrical model was divided into equal-spaced grid points in $r$ and $z$ directions denoted by $N_{\mathrm{r}}$ and $N_{\mathrm{z}}$ respectively as shown in Figure 4.2. This transformed each of them into a set of simultaneous ordinary differential equations.

The ordinary differential equations are stiff equations since there are different scales of the independent variable on which the dependent variables are changing. The numerical method used to solve the stiff equations is Semi-implicit extrapolation method (Bader and Deuflhard, 1983). It is robust and an excellent method for stiff problems. An adaptive step size control method is used in the algorithm to achieve the accuracy in the solution with minimum computational effort. 


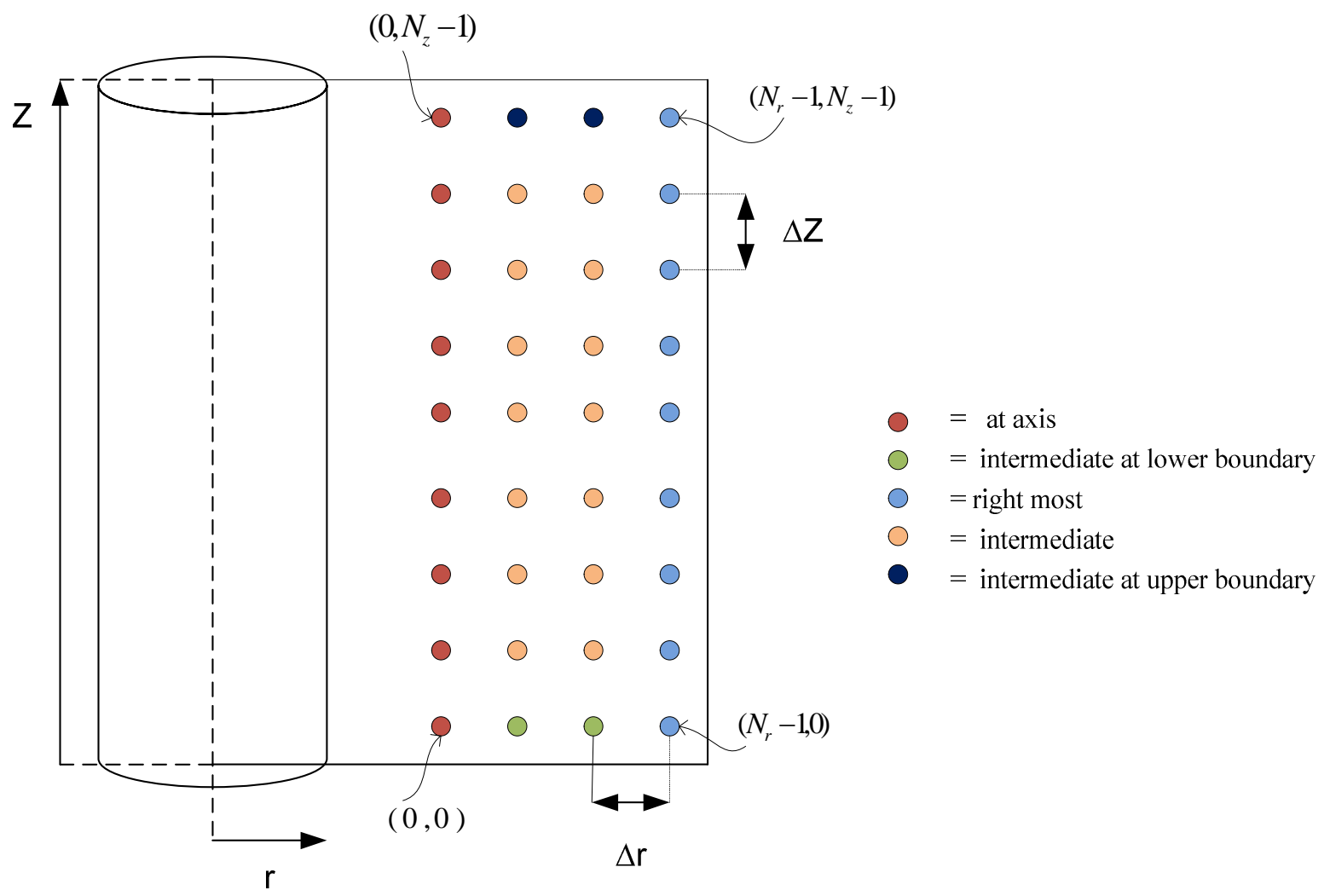

Figure 4.2 Cylindrical model and grid distribution

\subsubsection{Non-dimensionalization of the Model}

The numerical equations can be solved in much less time if the parameters and variables rescaled so that all computed quantities are of relatively similar scale. Thus, to solve the differential Equations (4.17) and (4.19) subject to the initial conditions in Equation (4.9) and the boundary conditions in Equation (4.10), it is more convenient to non-dimensionalize all the equations by introducing the following dimensionless variables:

$$
\left\{\begin{array}{ll}
\hat{r}=\frac{r}{R}, & \hat{z}=\frac{z}{z_{o}} \\
\widehat{D}=\frac{D}{D_{o}}, & \hat{Z}=\frac{Z}{z_{o}} \\
t_{o}=R^{2} / D_{o}, & \tau=\frac{t}{t_{o}}
\end{array}\right\}
$$


Here, $\hat{r}$ and $\hat{z}$ are the dimensionless radial and vertical coordinates, $\tau$ is the dimensionless time, $\widehat{D}$ is the dimensionless dispersion coefficient, $\hat{Z}$ is the dimensionless height of bitumen, and $D_{o}$ is a maximum dispersion value. With the above dimensionless variables, Equations (4.12) and (4.8) become:

$$
\begin{aligned}
& \frac{\partial \omega}{\partial \tau}=-3 \frac{\alpha t_{o}}{\varphi Z_{o}} \omega^{2} \frac{\partial \omega}{\partial \hat{z}}+\frac{D_{o} t_{o} \hat{D}}{R^{2}} \frac{1}{\hat{r}} \frac{\partial \omega}{\partial \hat{r}}+\frac{D_{o} t_{o}}{R^{2}} \frac{\partial \hat{D}}{\partial \omega}\left(\frac{\partial \omega}{\partial \hat{r}}\right)^{2}+\frac{D_{o} t_{o} \hat{D}}{R^{2}} \frac{\partial^{2} \omega}{\partial \hat{r}^{2}} \\
& \frac{\partial \hat{Z}}{\partial \tau}=-v \frac{t_{o}}{Z_{o}}
\end{aligned}
$$

Consequently, the initial and boundary conditions become:

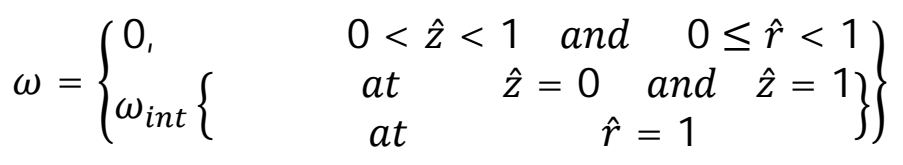

$$
\begin{aligned}
& \omega=\omega_{\text {int }}\left\{\begin{array}{lc}
0 \leq \hat{z} \leq Z / Z_{o} & \hat{r}=1 \\
0 \leq \hat{r}<1 & \hat{z}=0, \hat{z}=Z / Z_{o}
\end{array}\right.
\end{aligned}
$$

The resulting set of non-dimintionalized ordinary differential equations written for corresponding grid points are as follows:

\section{The Mathematical Model}

For intermediate grid points, i.e for $0<i<\left(N_{\mathrm{r}}-1\right)$ and $0<j<\left(N_{\mathrm{z}}-1\right)$

$$
\begin{aligned}
\frac{d \omega_{i, j}}{d \tau}= & \frac{D_{o} t_{o}}{R^{2}} \hat{D}\left[\frac{1}{\hat{r}_{i}} \frac{\omega_{i+1, j}-\omega_{i-1, j}}{2 \Delta \hat{r}}+\frac{\omega_{i+1, j}-2 \omega_{i, j}+\omega_{i-1, j}}{\Delta \hat{r}^{2}}\right]+ \\
& \frac{D_{o} t_{o}}{R^{2}} \frac{\partial \hat{D}}{\partial \omega}\left[\frac{\omega_{i+1, j}-\omega_{i-1, j}}{2 \Delta \hat{r}}\right]^{2}-3 \frac{K_{\mathrm{r}} K \rho g \cos \theta t_{o}}{\mu_{0} \varphi Z_{o}} \omega_{i, j}^{2}\left[\frac{\omega_{i, j+1}-\omega_{i, j-1}}{2 \Delta \hat{z}_{i}}\right]
\end{aligned}
$$


For axial grid points, i.e.

a. for $i=0$ and $0<j<\left(N_{\mathrm{z}}-1\right)$

$$
\frac{d \omega_{0, j}}{d \tau}=-3 \frac{K_{\mathrm{r}} K \rho g \cos \theta t_{o}}{\mu_{0} \varphi Z_{o}} \omega_{0, j}^{2}\left[\frac{\omega_{0, j+1}-\omega_{0, j-1}}{2 \Delta \hat{z}_{0}}\right]
$$

b. for $i=0$ and $j=0$

$$
\frac{d \omega_{0,0}}{d \tau}=-3 \frac{K_{\mathrm{r}} K \rho g \cos \theta t_{o}}{\mu_{0} \varphi Z_{o}} \omega_{0,0}^{2}\left[\frac{\omega_{0,1}-\omega_{\mathrm{int}}}{2 \Delta \hat{z}_{0}}\right]
$$

c. for $i=0$ and $j=\left(N_{\mathrm{z}}-1\right)$

$$
\frac{d \omega_{0, N_{z}-1}}{d \tau}=-3 \frac{K_{\mathrm{r}} K \rho g \cos \theta t_{o}}{\mu_{0} \varphi Z_{o}} \omega_{0, N_{\mathrm{z}}-1}^{2}\left[\frac{\omega_{\mathrm{int}}-\omega_{0, N_{\mathrm{z}}-2}}{2 \Delta \hat{z}_{0}}\right]
$$

For the right most grid points, i.e.

a. for $i=\left(N_{\mathrm{r}}-1\right)$ and $j=0$

$$
\begin{aligned}
\frac{d \omega_{N_{r}-1,0}}{d \tau} & =\frac{D_{o} t_{o}}{R^{2}} \hat{D}\left[\frac{1}{\hat{r}_{N_{r}-1}} \frac{\omega_{\mathrm{int}}-\omega_{N_{r}-2,0}}{2 \Delta \hat{r}}+\frac{\omega_{\mathrm{int}}-2 \omega_{N_{r}-1,0}+\omega_{N_{r}-2,0}}{\Delta \hat{r}^{2}}\right]+ \\
& \frac{D_{o} t_{o}}{R^{2}} \frac{\partial \hat{D}}{\partial \omega}\left[\frac{\omega_{\mathrm{int}}-\omega_{N_{r}-2,0}}{2 \Delta \hat{r}}\right]^{2}-3 \frac{K_{\mathrm{r}} K \rho g \cos \theta t_{o}}{\mu_{0} \varphi Z_{o}} \omega_{N_{r}-1,0}^{2}\left[\frac{\omega_{N_{r}-1,1}-\omega_{\mathrm{int}}}{2 \Delta \hat{z}_{N_{r}-1}}\right]
\end{aligned}
$$

b. for $i=\left(N_{\mathrm{r}}-1\right)$ and $0<j<\left(N_{\mathrm{z}}-1\right)$ 


$$
\begin{aligned}
\frac{d \omega_{N_{r}-1, j}}{d \tau} & =\frac{D_{o} t_{o}}{R^{2}} \hat{D}\left[\frac{1}{\hat{r}_{N_{r}-1}} \frac{\omega_{\mathrm{int}}-\omega_{N_{r}-2, j}}{2 \Delta \hat{r}}+\frac{\omega_{\mathrm{int}}-2 \omega_{N_{r}-1, j}+\omega_{N_{r}-2, j}}{\Delta \hat{r}^{2}}\right]+ \\
& \frac{D_{o} t_{o}}{R^{2}} \frac{\partial \hat{D}}{\partial \omega}\left[\frac{\omega_{\mathrm{int}}-\omega_{N_{r}-2, j}}{2 \Delta \hat{r}}\right]^{2}-3 \frac{K_{\mathrm{r}} K \rho g \cos \theta t_{o}}{\mu_{0} \varphi Z_{o}} \omega_{N_{r}-1, j}^{2}\left[\frac{\omega_{N_{r}-1, j+1}-\omega_{N_{r}-1, j-1}}{2 \Delta \hat{z}_{N_{r}-1}}\right]
\end{aligned}
$$

c. for $i=\left(N_{\mathrm{r}}-1\right)$ and $j=\left(N_{\mathrm{z}}-1\right)$

$$
\begin{aligned}
& \frac{d \omega_{N_{\mathrm{r}}-1, N_{z}-1}}{d \tau}=\frac{D_{o} t_{o}}{R^{2}} \hat{D}\left[\frac{1}{\hat{N}_{\mathrm{N}_{\mathrm{r}}-1}} \frac{\omega_{\mathrm{int}}-\omega_{N_{\mathrm{r}}-2, N_{\mathrm{z}}-1}}{2 \Delta \hat{r}}+\frac{\omega_{\mathrm{int}}-2 \omega_{N_{\mathrm{r}}-1, N_{z}-1}+\omega_{N_{\mathrm{r}}-2, N_{\mathrm{z}}-1}}{\Delta \hat{r}^{2}}\right]+ \\
& \frac{D_{o} t_{o}}{R^{2}} \frac{\partial \hat{D}}{\partial \omega}\left[\frac{\omega_{\mathrm{int}}-\omega_{N_{\mathrm{r}}-2, N_{z}-1}}{2 \Delta \hat{r}}\right]^{2}-3 \frac{K_{\mathrm{r}} K \rho g \cos \theta t_{o}}{\mu_{0} \varphi Z_{o}} \omega_{N_{\mathrm{r}}-1, N_{z}-1}^{2}\left[\frac{\omega_{\mathrm{int}}-\omega_{N_{\mathrm{r}}-1, N_{z}-2}}{2 \Delta \hat{z}_{N_{\mathrm{r}}-1}}\right]
\end{aligned}
$$

For the lower most intermediate grid points, i.e. for $0<i<\left(N_{\mathrm{r}}-1\right)$ and $j=0$

$$
\begin{aligned}
\frac{d \omega_{i, 0}}{d \tau}= & \frac{D_{o} t_{o}}{R^{2}} \hat{D}\left[\frac{1}{\hat{r}_{i}} \frac{\omega_{i+1,0}-\omega_{i-1,0}}{2 \Delta \hat{r}}+\frac{\omega_{i+1,0}-2 \omega_{i, 0}+\omega_{i-1,0}}{\Delta \hat{r}^{2}}\right]+ \\
& \frac{D_{o} t_{o}}{R^{2}} \frac{\partial \hat{D}}{\partial \omega}\left[\frac{\omega_{i+1,0}-\omega_{i-1,0}}{2 \Delta \hat{r}}\right]^{2}-3 \frac{K_{\mathrm{r}} K \rho \cos \theta t_{o}}{\mu_{0} \varphi Z_{o}} \omega_{i, 0}^{2}\left[\frac{\omega_{i, 1}-\omega_{\text {int }}}{2 \Delta \hat{z}_{i}}\right]
\end{aligned}
$$

For the upper most intermediate grid points, i.e. for $0<i<\left(N_{\mathrm{r}}-1\right)$ and $j=\left(N_{\mathrm{z}}-1\right)$

$$
\begin{aligned}
\frac{d \omega_{i, N_{z}-1}}{d \tau}= & \frac{D_{o} t_{o}}{R^{2}} \hat{D}\left[\frac{1}{\hat{r}_{i}} \frac{\omega_{i+1, N_{\mathrm{z}}-1}-\omega_{\mathrm{I}-1, N_{\mathrm{z}}-1}}{2 \Delta \hat{r}}+\frac{\omega_{i+1, N_{\mathrm{z}}-1}-2 \omega_{i, N_{\mathrm{z}}-1}+\omega_{i-1, N_{\mathrm{z}}-1}}{\Delta \hat{r}^{2}}\right]+ \\
& \frac{D_{o} t_{o}}{R^{2}} \frac{\partial \hat{D}}{\partial \omega}\left[\frac{\omega_{i+1, N_{\mathrm{z}}-1}-\omega_{i-1, N_{\mathrm{z}}-1}}{2 \Delta \hat{r}}\right]^{2}-3 \frac{K_{\mathrm{r}} K \rho g \cos \theta t_{o}}{\mu_{0} \varphi Z_{o}} \omega_{i, N_{\mathrm{z}}-1}^{2}\left[\frac{\omega_{\mathrm{int}}-\omega_{i, N_{\mathrm{z}}-2}}{2 \Delta \hat{z}_{i}}\right]
\end{aligned}
$$


Where $\omega_{i, j}$ is the mass fraction of the solvent at the node, $(i, j)$ corresponding to the coordinate, $\hat{r}$ and $\hat{z}$. The distances between grid points are $\Delta \hat{r}$ and $\Delta \hat{Z}_{i}$ respectively along $\hat{r}$ and $\hat{z}$ directions. $\Delta \hat{r}$ is constant and given by

$$
\Delta \hat{r}=\frac{1}{N_{\mathrm{r}}}
$$

but $\Delta \hat{Z}_{i}$ varies with time along $\hat{r}$ direction, and is given by

$$
\Delta \hat{Z}_{i}=\frac{\hat{Z}_{i}}{N_{\mathrm{z}}+1}
$$

The change of the bitumen height at any time is given by the following equations:

The change in height for $0 \leq i<\left(N_{\mathrm{r}}-1\right)$ is given by

$$
\frac{d \hat{Z}_{i}}{d \tau}=-\frac{t_{o}}{Z_{o}} v_{i}=-\frac{t_{o}}{Z_{o}} \frac{K_{\mathrm{r}} K \rho g \cos \theta}{\mu_{0}}\left[\frac{\omega_{i, 0}+\omega_{i+1,0}+2 \omega_{\mathrm{int}}}{4}\right]^{2}
$$

and for $i=N_{\mathrm{r}}-1$

$$
\frac{d \hat{Z}_{N_{\mathrm{r}}-1}}{d \tau}=-\frac{t_{o}}{Z_{o}} v_{N_{\mathrm{r}}-1}=-\frac{t_{o}}{Z_{o}} \frac{K_{\mathrm{r}} K \rho g \cos \theta}{\mu_{0}}\left[\frac{\omega_{N_{\mathrm{r}}-1,0}+3 \omega_{\mathrm{int}}}{4}\right]^{2}
$$

Similarly, introducing the following dimensionless variables: 


$$
\left\{\begin{array}{ccc}
\hat{\lambda}=\frac{\lambda}{\lambda_{o}} & , & \hat{\gamma}=\frac{\gamma}{\gamma_{o}}, \\
\hat{\rho}=\frac{\rho}{\rho_{o}}, & \hat{m}=\frac{m}{m_{o}} \\
\gamma_{o}=t_{o} \rho_{o} m_{o} R & , & \gamma_{o}=\lambda_{o}
\end{array}\right\}
$$

where $\hat{\lambda}$ is the first dimensionless adjoint variable, $\hat{\gamma}$ is the second dimensionless adjoint variable, $\hat{\rho}$ is the dimensionless live oil density, $\widehat{m}$ is the dimensionless cumulative produced live oil mass, $\rho_{o}$ is an arbitrary live oil density, $m_{o}$ is the maximum cumulative produced live oil mass.

Equation (4.50), Equation (4.48) and Equation (4.46) become:

$\frac{\partial \hat{\lambda}}{\partial \tau}=-3 \frac{\alpha t_{o}}{\varphi Z_{o}} \omega^{2} \frac{\partial \hat{\lambda}}{\partial \hat{z}}-\frac{D_{o} t_{o} \hat{D}}{R^{2}} \frac{\hat{\lambda}}{\hat{r}^{2}}+\frac{D_{o} t_{o}}{R^{2}} \frac{\hat{D}}{\hat{r}} \frac{\partial \hat{\lambda}}{\partial \hat{r}}-\frac{D_{o} t_{o} \hat{D}}{R^{2}} \frac{\partial^{2} \hat{\lambda}}{\partial \hat{r}^{2}}$

$\frac{\partial \hat{\gamma}}{\partial \tau}=-4 \pi \frac{t_{o}}{\gamma_{o}} \rho_{o} m_{o} R \hat{\rho} \varphi\left(\hat{m}_{c}-\hat{m}_{e}\right) \hat{r}$

$J_{D}=-\frac{\lambda_{o}}{R^{2}} \frac{\hat{\lambda}}{\hat{r}} \frac{\partial \omega}{\partial \hat{r}}-\frac{\lambda_{o}}{R^{2}} \hat{\lambda} \frac{\partial^{2} \omega}{\partial \hat{r}^{2}}$

The resulting set of non-dimintionalized ordinary differential equations written for corresponding grid points are as follows:

\section{The Adjoint Equations}

For intermediate grid points, i.e for $0<i<\left(N_{\mathrm{r}}-1\right)$ and $0<j<\left(N_{\mathrm{z}}-1\right)$

$$
\begin{aligned}
\frac{d \hat{\lambda}_{i, j}}{d \tau}= & \frac{D_{o} t_{o}}{R^{2}} \hat{D}\left[\frac{1}{\hat{r}_{i}} \frac{\hat{\lambda}_{i+1, j}-\hat{\lambda}_{i-1, j}}{2 \Delta \hat{r}}-\frac{\hat{\lambda}_{i+1, j}-2 \hat{\lambda}_{i, j}+\hat{\lambda}_{i-1, j}}{\Delta \hat{r}^{2}}\right]- \\
& \frac{D_{o} t_{o}}{R^{2}} \hat{D}\left[\frac{\hat{\lambda}_{i, j}}{\hat{r}_{i}^{2}}\right]-3 \frac{K_{\mathrm{r}} K \rho g \cos \theta t_{o}}{\mu_{0} \varphi Z_{o}} \omega_{i, j}^{2}\left[\frac{\hat{\lambda}_{i, j+1}-\hat{\lambda}_{i, j-1}}{2 \Delta \hat{z}_{i}}\right]
\end{aligned}
$$


For axial grid points, i.e. $i=0$ and $0<j<\left(N_{\mathrm{z}}-1\right)$

$$
\frac{d \hat{\lambda}_{0,0}}{d \tau}=\frac{d \hat{\lambda}_{0, j}}{d \tau}=\frac{d \hat{\lambda}_{0, N_{z}-1}}{d \tau}=0
$$

For the right most grid points, i.e.

a. for $i=\left(N_{\mathrm{r}}-1\right)$ and $j=0$

$$
\begin{aligned}
\frac{d \hat{\lambda}_{N_{\mathrm{r}}-1,0}}{d \tau}= & \frac{D_{o} t_{o}}{R^{2}} \hat{D}\left[\frac{1}{\hat{r}_{N_{\mathrm{r}}-1}} \frac{-\hat{\lambda}_{N_{\mathrm{r}}-2,0}}{2 \Delta \hat{r}}-\frac{-2 \hat{\lambda}_{N_{\mathrm{r}}-1,0}+\hat{\lambda}_{N_{\mathrm{r}}-2,0}}{\Delta \hat{r}^{2}}\right]- \\
& \frac{D_{o} t_{o}}{R^{2}} \hat{D}\left[\frac{\hat{\lambda}_{N_{\mathrm{r}}-1,0}}{\hat{r}_{N_{\mathrm{r}}-1}^{2}}\right]-3 \frac{K_{\mathrm{r}} K \rho g \cos \theta t_{o}}{\mu_{o} \varphi Z_{o}} \omega_{N_{\mathrm{r}}-1,0}^{2}\left[\frac{\hat{\lambda}_{N_{\mathrm{r}}-1,1}}{2 \Delta{\hat{N_{N}}}_{N_{\mathrm{r}}}}\right]
\end{aligned}
$$

b. for $i=\left(N_{\mathrm{r}}-1\right)$ and $0<j<\left(N_{\mathrm{z}}-1\right)$

$$
\begin{aligned}
\frac{d \hat{\lambda}_{N_{\mathrm{r}}-1, j}}{d \tau}= & \frac{D_{o} t_{o}}{R^{2}} \hat{D}\left[\frac{1}{\hat{r}_{N_{\mathrm{r}}-1}} \frac{-\hat{\lambda}_{N_{\mathrm{r}}-2, j}}{2 \Delta \hat{r}}-\frac{-2 \hat{\lambda}_{N_{\mathrm{r}}-1, j}+\hat{\lambda}_{N_{\mathrm{r}}-2, j}}{\Delta \hat{r}^{2}}\right]- \\
& \frac{D_{o} t_{o}}{R^{2}} \hat{D}\left[\frac{\hat{\lambda}_{N_{\mathrm{r}}-1, j}}{\hat{r}_{N_{\mathrm{r}}-1}^{2}}\right]-3 \frac{K_{\mathrm{r}} K \rho g \cos \theta t_{o}}{\mu_{0} \varphi Z_{o}} \omega_{N_{\mathrm{r}}-1, j}^{2}\left[\frac{\hat{\lambda}_{N_{\mathrm{r}}-1, j}-\hat{\lambda}_{N_{\mathrm{r}}-1, j-1}}{2 \Delta \hat{z}_{N_{\mathrm{r}}-1}}\right]
\end{aligned}
$$

c. for $i=\left(N_{\mathrm{r}}-1\right)$ and $j=\left(N_{\mathrm{z}}-1\right)$ 


$$
\begin{gathered}
\frac{d \hat{\lambda}_{N_{\mathrm{r}}-1, N_{\mathrm{z}}-1}}{d \tau}=\frac{D_{o} t_{o}}{R^{2}} \hat{D}\left[\frac{1}{\hat{r}_{N_{\mathrm{r}}-1}} \frac{-\hat{\lambda}_{N_{\mathrm{r}}-2, N_{\mathrm{z}}-1}}{2 \Delta \hat{r}}-\frac{-2 \hat{\lambda}_{N_{\mathrm{r}}-1, N_{\mathrm{z}}-1}+\hat{\lambda}_{N_{\mathrm{r}}-2, N_{\mathrm{z}}-1}}{\Delta \hat{r}^{2}}\right]- \\
\frac{D_{o} t_{o}}{R^{2}} \hat{D}\left[\frac{\hat{\lambda}_{N_{\mathrm{r}}-1, N_{\mathrm{z}}-1}}{\hat{r}_{N_{\mathrm{r}}-1}^{2}}\right]-3 \frac{K_{\mathrm{r}} K \rho g \cos \theta t_{o}}{\mu_{0} \varphi Z_{o}} \omega_{N_{\mathrm{r}}-1, N_{\mathrm{z}}-1}^{2}\left[\frac{-\hat{\lambda}_{N_{\mathrm{r}}-1, N_{\mathrm{z}}-2}}{2 \Delta \hat{z}_{N_{\mathrm{r}}-1}}\right]
\end{gathered}
$$

For the lower most intermediate grid points, i.e. for $0<i<\left(N_{\mathrm{r}}-1\right)$ and $j=0$

$$
\begin{aligned}
\frac{d \hat{\lambda}_{i, 0}}{d \tau}= & \frac{D_{o} t_{o}}{R^{2}} \hat{D}\left[\frac{1}{\hat{r}_{i}} \frac{\hat{\lambda}_{i+1,0}-\hat{\lambda}_{i-1,0}}{2 \Delta \hat{r}}-\frac{\hat{\lambda}_{i+1,0}-2 \hat{\lambda}_{i, 0}+\hat{\lambda}_{i-1,0}}{\Delta \hat{r}^{2}}\right]- \\
& \frac{D_{o} t_{o}}{R^{2}} \hat{D}\left[\frac{\hat{\lambda}_{i, 0}}{\hat{r}_{i}^{2}}\right]-3 \frac{K_{\mathrm{r}} K \rho g \cos \theta t_{o}}{\mu_{0} \varphi Z_{o}} \omega_{i, 0}^{2}\left[\frac{\hat{\lambda}_{i, 1}}{2 \Delta \hat{z}_{i}}\right]
\end{aligned}
$$

For the upper most intermediate grid points, i.e. for $0<i<\left(N_{\mathrm{r}}-1\right)$ and $j=\left(N_{\mathrm{z}}-1\right)$

$$
\begin{aligned}
\frac{d \hat{\lambda}_{i, N_{z}-1}}{d \tau}= & \frac{D_{o} t_{o}}{R^{2}} \hat{D}\left[\frac{1}{\hat{r}_{i}} \frac{\hat{\lambda}_{i+1, N_{z}-1}-\hat{\lambda}_{i-1, N_{z}-1}}{2 \Delta \hat{r}}-\frac{\hat{\lambda}_{i+1, N_{z}-1}-2 \hat{\lambda}_{i, N_{z}-1}+\hat{\lambda}_{i-1, N_{z}-1}}{\Delta \hat{r}^{2}}\right]- \\
& \frac{D_{o} t_{o}}{R^{2}} \hat{D}\left[\frac{\hat{\lambda}_{i, N_{z}-1}}{\hat{r}_{i}^{2}}\right]-3 \frac{K_{\mathrm{r}} K \rho g \cos \theta t_{o}}{\mu_{0} \varphi Z_{o}} \omega_{i, N_{z}-1}^{2}\left[\frac{-\hat{\lambda}_{i, N_{z}-2}}{2 \Delta \hat{z}_{i}}\right]
\end{aligned}
$$

The equation for the second adjoint variable is

For $0 \leq i<\left(N_{\mathrm{r}}-1\right)$

$$
\frac{d \hat{\gamma}_{i}}{d \tau}=-4 \pi \varphi \rho_{o} \frac{t_{o}}{\gamma_{o}} m_{o} \hat{\rho} R \hat{r}_{i+1}\left[\hat{m}_{\mathrm{c}}-\hat{m}_{\mathrm{e}}\right]
$$


Similarly, the gradient correction $J_{D}$ was expressed in a finite-difference form along $r$ direction. The resulting non-dimintionalized equations written for corresponding grid points are as follows: For intermediate grid points, i.e for $0<i<\left(\mathrm{N}_{\mathrm{r}}-1\right) \quad$ and $\quad 0<j<\left(\mathrm{N}_{\mathrm{z}}-1\right)$

$\left(\frac{\partial J}{\partial D}\right)_{i, j}=-\frac{\lambda_{o}}{R^{2}} \hat{\lambda}_{i, j}\left[\frac{1}{\hat{r}_{i}} \frac{\omega_{i+1, j}-\omega_{i-1, j}}{2 \Delta \hat{r}}\right]-\frac{\lambda_{o}}{R^{2}} \hat{\lambda}_{i, j}\left[\frac{\omega_{i+1, j}-2 \omega_{i, j}+\omega_{i-1, j}}{\Delta \hat{r}^{2}}\right]$

For axial grid points, i.e. $i=0$ and $0 \leq j \leq\left(N_{\mathrm{z}}-1\right)$

$\left(\frac{\partial J}{\partial D}\right)_{0,0}=\left(\frac{\partial J}{\partial D}\right)_{0, j}=\left(\frac{\partial J}{\partial D}\right)_{0, N_{\mathrm{z}}-1}=0$

For the right most grid points, i.e.

a. for $i=\left(N_{\mathrm{r}}-1\right)$ and $j=0$

$\left(\frac{\partial J}{\partial D}\right)_{N_{\mathrm{r}}-1,0}=-\frac{\lambda_{o}}{R^{2}} \hat{\lambda}_{N_{\mathrm{r}}-1,0}\left[\frac{1}{\hat{r}_{N_{\mathrm{r}}-1}} \frac{\omega_{\text {int }}-\omega_{N_{\mathrm{r}}-2,0}}{2 \Delta \hat{r}}\right]-\frac{\lambda_{o}}{R^{2}} \hat{\lambda}_{N_{\mathrm{r}}-1,0}\left[\frac{\omega_{\text {int }}-2 \omega_{N_{\mathrm{r}}-1,0}+\omega_{N_{\mathrm{r}}-2,0}}{\Delta \hat{r}^{2}}\right]$

b. for $i=\left(N_{\mathrm{r}}-1\right)$ and $0<j<\left(N_{\mathrm{z}}-1\right)$

$\left(\frac{\partial J}{\partial D}\right)_{N_{\mathrm{r}}-1, j}=-\frac{\lambda_{o}}{R^{2}} \hat{\lambda}_{N_{\mathrm{r}}-1, j}\left[\frac{1}{\hat{r}_{N_{\mathrm{r}}-1}} \frac{\omega_{\mathrm{int}}-\omega_{N_{\mathrm{r}}-2, j}}{2 \Delta \hat{r}}\right]-\frac{\lambda_{o}}{R^{2}} \hat{\lambda}_{N_{\mathrm{r}}-1, j}\left[\frac{\omega_{\text {int }}-2 \omega_{N_{\mathrm{r}}-1, j}+\omega_{N_{\mathrm{r}}-2, j}}{\Delta \hat{r}^{2}}\right]$

c. for $i=\left(N_{\mathrm{r}}-1\right)$ and $j=\left(N_{\mathrm{z}}-1\right)$

$\left(\frac{\partial J}{\partial D}\right)_{N_{\mathrm{r}}-1, N_{z}-1}=-\frac{\lambda}{R^{2}} \hat{\lambda}_{N_{\mathrm{r}}-1, N_{z}-1}\left[\frac{1}{\hat{r}_{N_{\mathrm{r}}-1}} \frac{\omega_{\mathrm{int}}-\omega_{N_{\mathrm{r}}-2, N_{z}-1}}{2 \Delta \hat{r}}\right]-\frac{\lambda_{o}}{R^{2}} \hat{\lambda}_{N_{\mathrm{r}}-1, N_{z}-1}\left[\frac{\omega_{\mathrm{int}}-2 \omega_{N_{\mathrm{r}}-1, N_{z}-1}+\omega_{N_{\mathrm{r}}-2, N_{z}-1}}{\Delta \hat{r}^{2}}\right]$ 
For the lower most intermediate grid points, i.e. for $0<i<\left(N_{\mathrm{r}}-1\right)$ and $j=0$

$\left(\frac{\partial J}{\partial D}\right)_{i, 0}=-\frac{\lambda_{o}}{R^{2}} \hat{\lambda}_{i, 0}\left[\frac{1}{\hat{r}_{i}} \frac{\omega_{i+1,0}-\omega_{i-1,0}}{2 \Delta \hat{r}}\right]-\frac{\lambda_{o}}{R^{2}} \hat{\lambda}_{i, 0}\left[\frac{\omega_{i+1,0}-2 \omega_{i, 0}+\omega_{i-1,0}}{\Delta \hat{r}^{2}}\right]$

For the upper most intermediate grid points, i.e. for $0<i<\left(N_{\mathrm{r}}-1\right)$ and $j=\left(N_{\mathrm{z}}-1\right)$

$\left(\frac{\partial J}{\partial D}\right)_{i, N_{\mathrm{z}}-1}=-\frac{\lambda_{o}}{R^{2}} \hat{\lambda}_{i, N_{\mathrm{z}}-1}\left[\frac{1}{\hat{r}_{i}} \frac{\omega_{i+1, N_{\mathrm{z}}-1}-\omega_{i-1, N_{\mathrm{z}}-1}}{2 \Delta \hat{r}}\right]-\frac{\lambda_{o}}{R^{2}} \hat{\lambda}_{i, N_{\mathrm{z}}-1}\left[\frac{\omega_{i+1, N_{\mathrm{z}}-1}-2 \omega_{i, N_{\mathrm{z}}-1}+\omega_{i-1, N_{\mathrm{z}}-1}}{\Delta \hat{r}^{2}}\right]$

The cumulative mass of produced live oil at any time becomes:

$m_{c}=2 \pi \varphi \rho Z_{o} R^{2} \sum_{i=0}^{N_{i}-1}\left(1-\hat{Z}_{i}\right) \hat{r}_{i+1} \Delta \hat{r}$

The adjoint equations have to be solved backward in the time domain. Since they are dependent on concentration and height, they can be only solved after solving the equation of continuity forward in the time domain. With an accuracy of $10^{-6}$ in the time domain, the equations were numerically integrated using semi-implicit Bader-Deuflhard algorithm, and adaptive step size control (Press et al., 2002). Analytical Jacobian of Equations (4.63-4.71), (4.74), (4.75), (4.804.86) and (4.87) was employed in the calculations, and the validity of the Jacobian equations was confirmed with the analytical solution to an error of $10^{-4}$. To fix the number of grid points along the $r$ and $z$ directions, $N_{\mathrm{r}}$ and $N_{\mathrm{z}}$, the equations were integrated with increasing the number of grid points until the changes in the calculated oil production rates became negligible. The number of grid points along the $r$ and $z$ directions, were chosen to provide reasonable computation times. 


\subsubsection{The Optimal Control Algorithm}

Based on the necessary conditions for the minimum of $J$, the following computational algorithm was developed to determine the concentration-dependent solvent dispersion function by iterative minimization of $J$ :

1. Initialize dispersion function.

2. Simultaneously integrate the continuity equation [Equation (4.59)] and Equation (4.60), subject to the initial and boundary conditions, to obtain the values of $\omega(t, r, z)$ and $Z(t, r)$ for each node.

3. Calculate the objective functional given by Equation (4.15).

4. Simultaneously integrate Equation (4.77) and Equation (4.78) backward, subject to the final boundary conditions, using stored values of $\omega$ and $Z$ to get the values of $\lambda(t, r, z)$ and $\gamma(t, r)$ for each grid point.

5. Improve $D(\omega)$ using the gradient correction given by Equation (4.79).

6. Go to Step 2 until the improvement in $J$ is negligible.

Figure 4.3 shows the optimal control algorithm developed to determine propane dispersion function. Programmed in $\mathrm{C}++$ language, the developed algorithm was implemented on Itanium 2/ Intel Itanium processor (64 bit, 1.5 GHz, 15.9 GB of RAM) with Intel C++ compiler. The above algorithm is computationally very intensive because a large number of finite-differenced ordinary differential equations and the associated Jacobian evaluations are needed to obtain reliable solutions. 


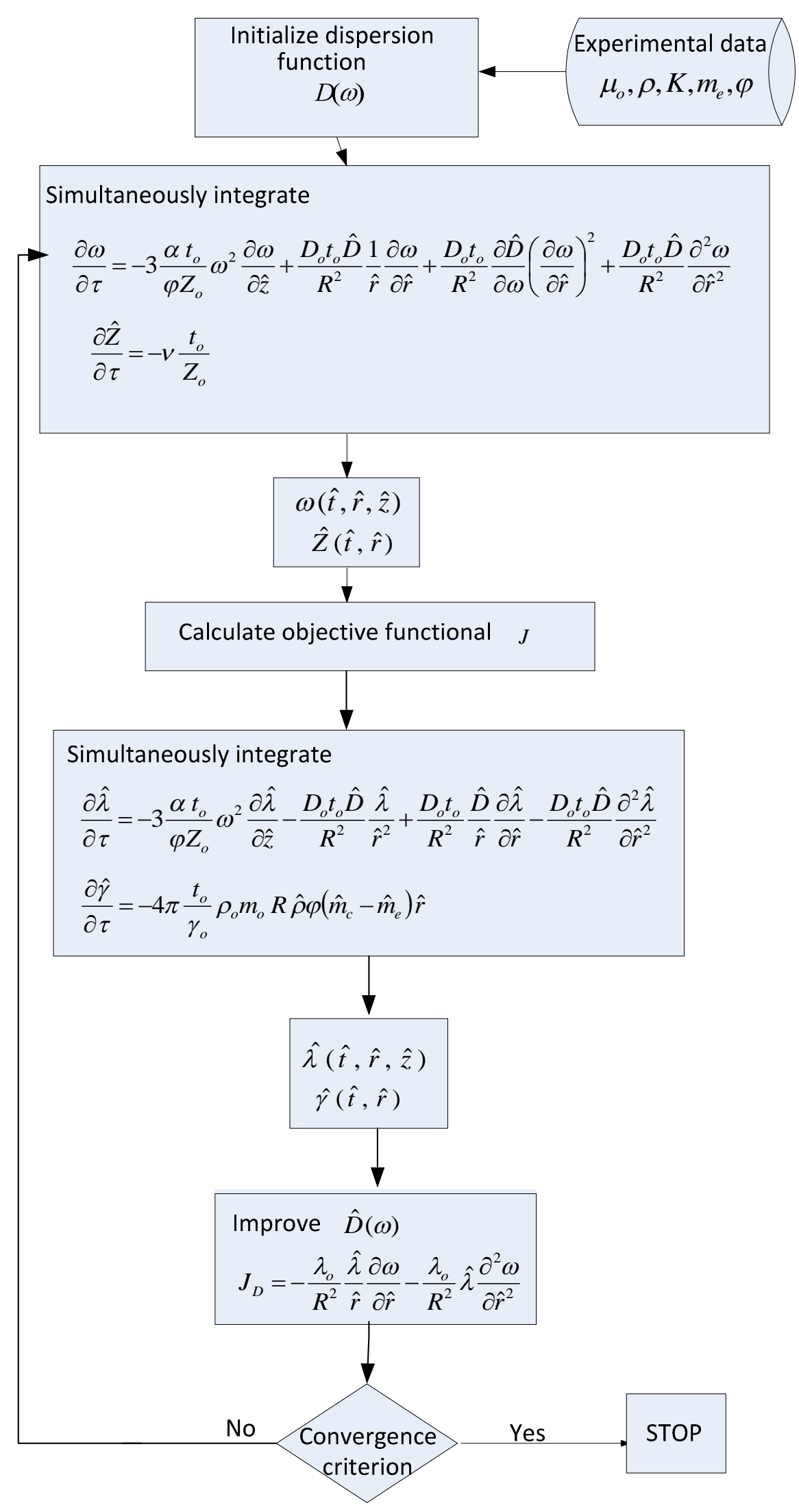

Figure 4.3 The Optimal Control algorithm 


\subsubsection{Initialization of the Algorithm Variables}

To start the minimization of the objective functional $J$, it is essential to have an initial guess for the optimization variable $D(\omega)$ and the solvent-heavy oil interface concentration $\omega_{\text {int }}$.

Figure 4.4 shows the Vapex interface. The first portion is the solvent chamber, where the mass fraction of the solvent is one when using a pure solvent. The third portion is the portion of heavy oil and bitumen with dissolved solvent. The second portion is the draining live oil portion. The draining live oil portion is the region where the viscosity of the live oil is reduced to the point that it becomes mobile and drains. The driving force for molecular diffusion is the difference between the interfacial concentration, i.e. the amount of solvent dissolved in heavy oil and bitumen, which is pressure and temperature dependent $\left(\omega_{\text {int }}\right)$, and the solvent concentration within the heavy oil and bitumen. The solvent concentration decreases from a maximum at solvent-live oil interface $\left(\omega_{\text {int }}\right)$ to a minimum in bitumen portion as shown in the solvent concentration profile in Figure 4.4.
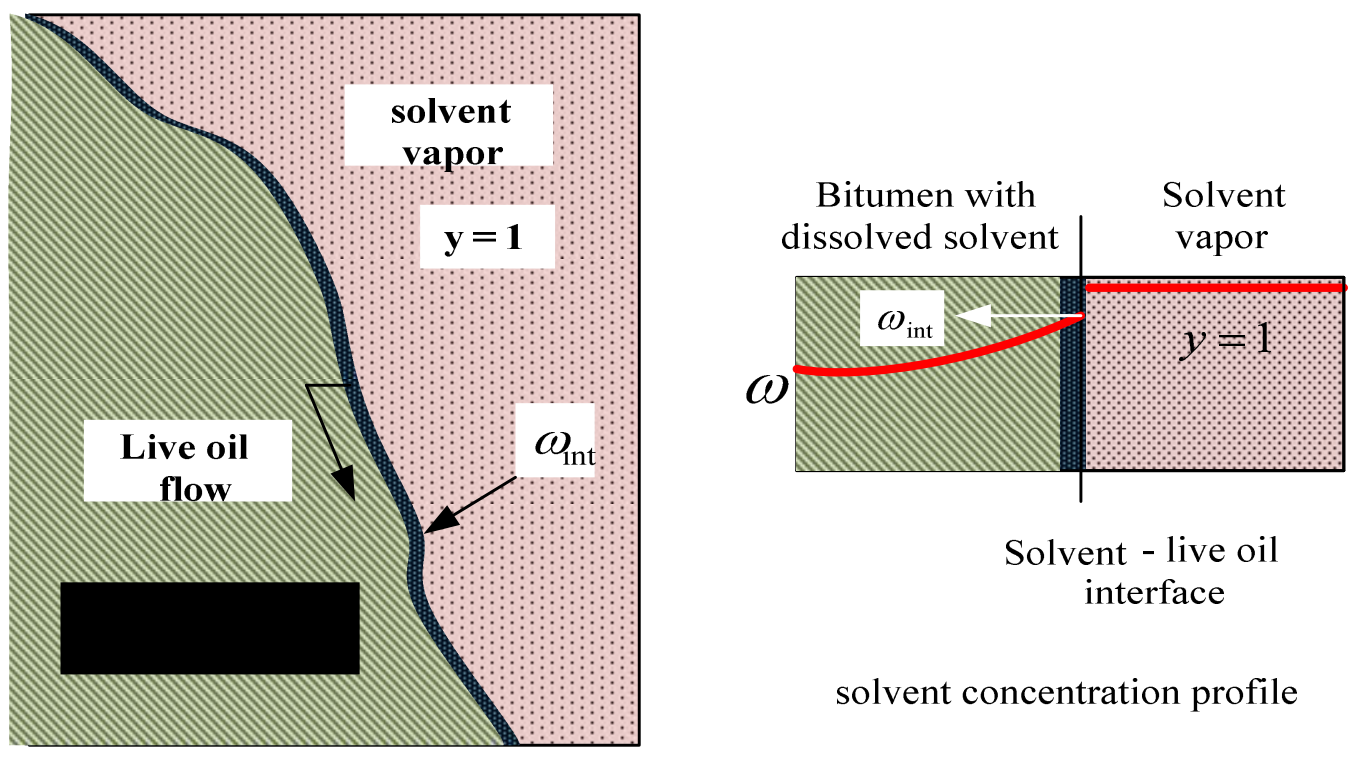

Figure 4.4 Solvent-heavy oil interface 
In the Vapex experiments, the draining live oil-solvent interface $\left(\omega_{\text {int }}\right)$ mass fraction is unknown. Therefore, propane mass fraction $\left(\omega_{\text {int }}\right)$ at the interface was optimally determined during Vapex experiments (see Chapter 5, Section 5.2.2).

The dispersion function was considered a discrete function at specified gas mass fractions between zero and the maximum concentration of the solvent in bitumen for an experiment. $D(\omega)$ was initialized to a uniform value as high as possible without causing $m_{\mathrm{c}}(t)$ to intersect $m_{\mathrm{e}}(t)$. 


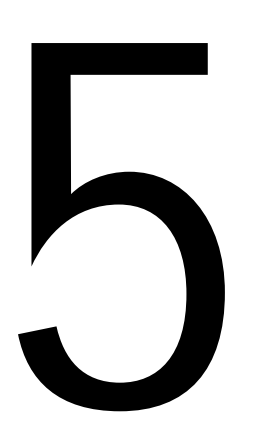

\section{Results and Discussion}

In this chapter, the results of the Vapex experiments conducted with various physical models and the numerical results will be presented and discussed. The effect of two main parameters on propane dispersion coefficient is investigated. The first parameter is the average grain size that is related to the permeability of the porous medium. The second parameter is the drainage height. In the laboratory experiments, the drainage height is identical to the height of the physical model. As mentioned previously, experiments were conducted in a laboratory scale physical model at $21^{\circ} \mathrm{C}$ temperature and $0.79 \mathrm{MPa}$ pressure. Under these conditions, the cumulative production mass of live oil was recorded. The average of live oil viscosity, density and propane mass fraction in heavy oil samples were determined.

\subsection{Experimental Results and Discussion}

Oil recovery from a simulated heavy oil-glass beads medium was carried out using three different glass beads sizes with three different heights of the cylindrical shape physical model. The permeabilities of the glass-beads packs used in the experiments were 204, 102, and 51 Darcy, while the heights of the physical model were 25,35 , and $45 \mathrm{~cm}$.

The physical model filled with heavy oil-glass beads saturated mixture was hanged to a load cell at the top of the pressure vessel. The load cell recorded the reduction in the mass of the physical model with time as the oil diluted by the dispersed solvent vapor and drained away by the action of gravity. This mass reduction was recorded by the data acquisition system and cumulative live

oil production was calculated from the load cell data recorded. To validate the experimental live 
oil production rate each experiment was performed twice and the percentage error between the two experiments was found to be an average of $2.5 \%$. A sample graph of recorded mass of the physical model with time throughout an experiment is found in Appendix D.

\subsubsection{System Pressure and Temperature}

Figure 5.1 presents the temperature of the pressure vessel and the water bath throughout an experiment (35 cm drainage height and 204 Darcy permeability physical model). The relative deviations of the temperatures of the pressure vessel and the water bath lie between $\pm 2 \%$, which indicates a relatively good temperature control using the temperature control system. A figure showing the relative deviations is found in Appendix E.

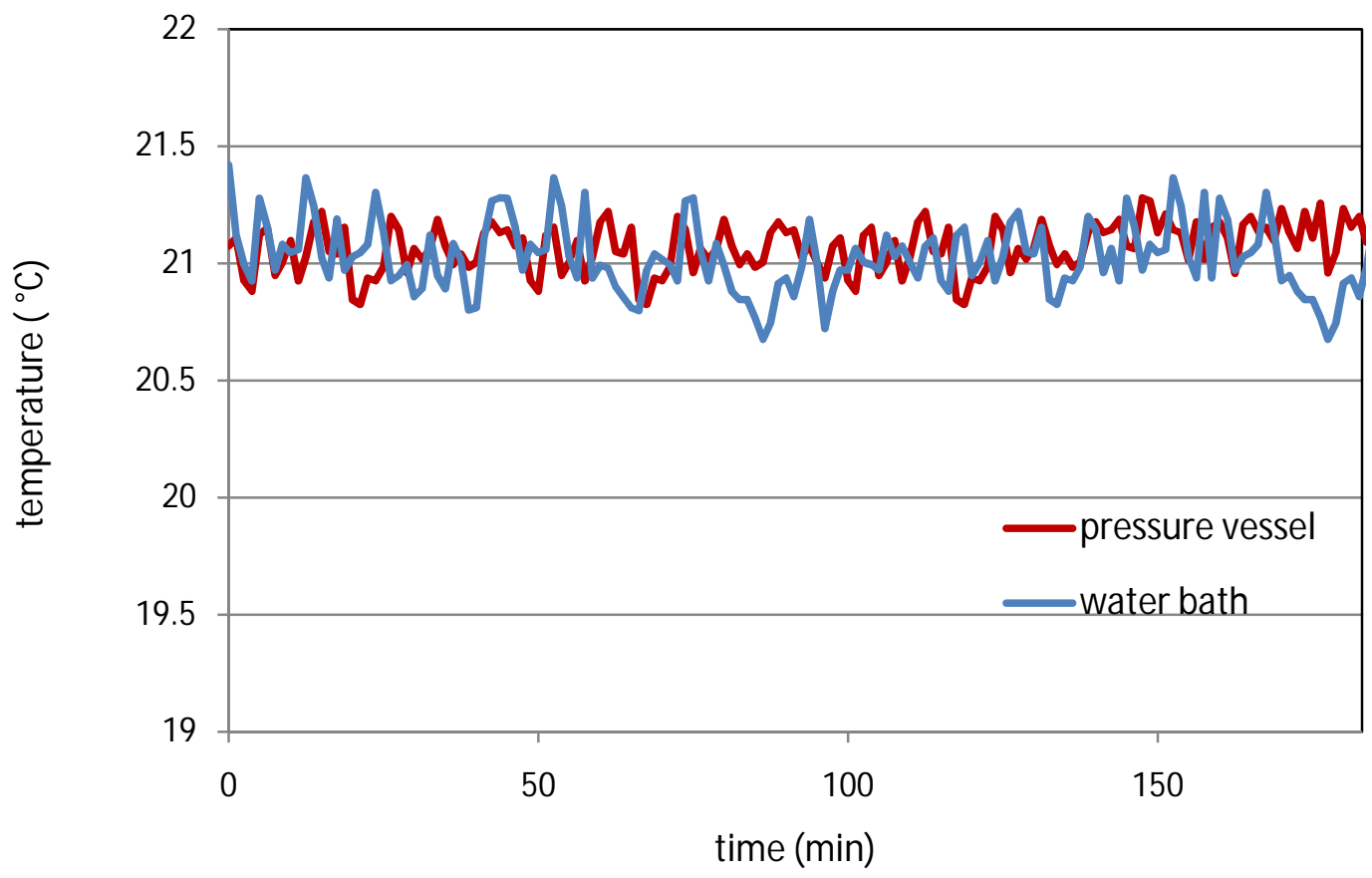

Figure 5.1 Temperature profile for the pressure vessel and the water bath (204 Darcy and $35 \mathrm{~cm})$.

The injection pressure was controlled through the pressure regulator installed on the propane supply cylinder. Figure 5.2 shows the pressure profile of the pressure vessel throughout an experiment. The vessel quickly pressurizes to the set pressure. It shows that within the first five 
minutes it reaches $90 \%$ of the maximum value. Then, the vessel gradually builds up and approaches a constant value.

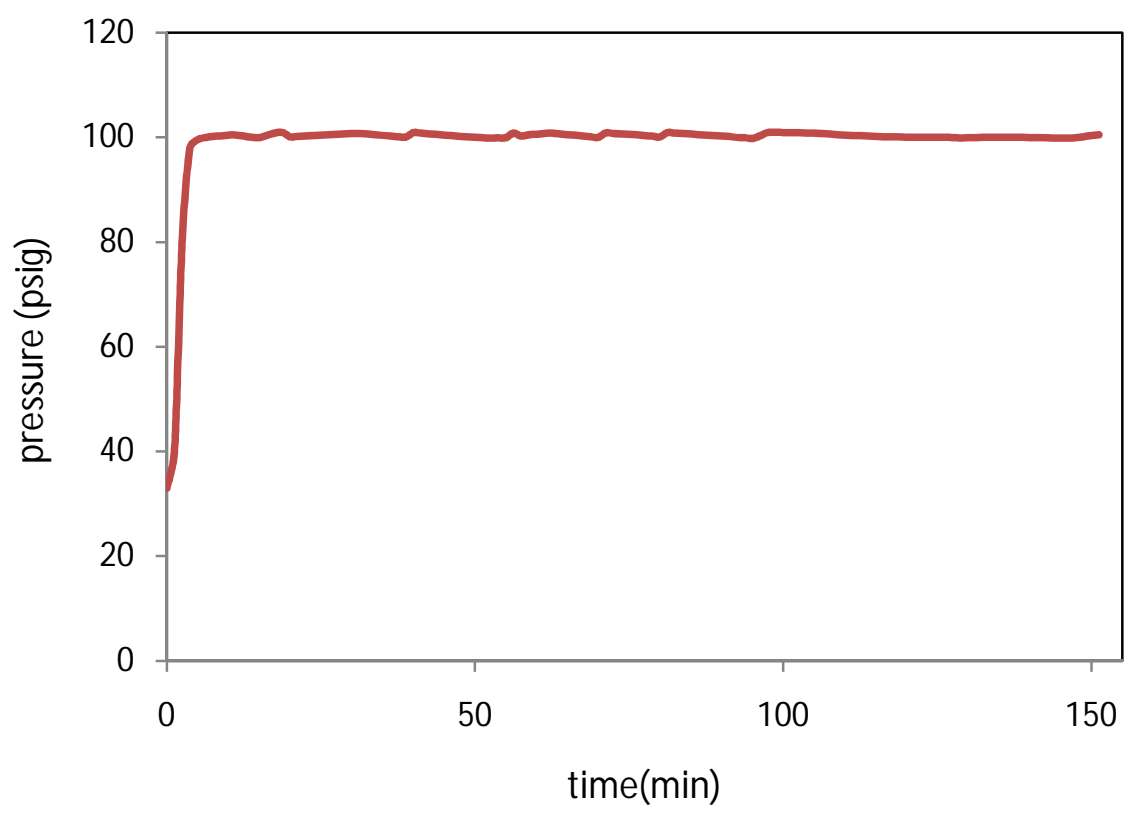

Figure 5.2 Vessel pressure versus time $(35 \mathrm{~cm}, 204$ Darcy)

\subsubsection{Live Oil Properties}

To get reliable results for Vapex modeling, the live oil viscosity data, live oil density values, and dissolved propane fraction were measured using live oil produced in the Vapex runs.

\subsubsection{Propane Solubility}

The amount of dissolved propane in the produced oil (propane mass fraction) was determined from the measured volume of the flashed gas and the weight of residual dead oil as explained in Section 3.7. Table 5.1 shows the averaged experimental values of propane mass fraction of Vapex experiments (8-10 live oil collections for each experiment). Propane mass fraction is approaching a higher value with lower packs permeability. This trend is also seen in results of Das (1995) experiments, which showed a permeability dependence of the solvent concentration. This dependence phenomenon is justified by the postulation of enhanced mass transfer, 
interfacial area, and larger residence time, which in turn lead to higher average propane mass fraction in the live oil.

A drainage height dependence of propane mass fraction is apparent. Propane mass fraction increases with higher drainage heights. Propane concentration at propane-bitumen interface is higher than the average propane concentration in the draining oil. Thus, as the live oil drains along the interface, it is still exposed to propane vapor and propane concentration in the flowing oil increases along the length towards the production end.

Table 5.1 Average propane mass fraction in produced oil for various physical models

\begin{tabular}{l|c|c|c}
\hline Permeability & \multicolumn{3}{|c}{ Model height } \\
\hline \multirow{2}{*}{ BT4 (204 Darcy) } & $25 \mathrm{~cm}$ & $35 \mathrm{~cm}$ & $45 \mathrm{~cm}$ \\
\cline { 2 - 4 } BT5 (102 Darcy) & 0.285 & 0.329 & 0.384 \\
BT6 (51 Darcy) & 0.336 & 0.371 & 0.421 \\
\hline
\end{tabular}

The average experimental amount of propane dissolved in the heavy oil falls in the range of observed propane solubility measured in the laboratory experiments by other investigators (Bademchi-Zadeh et al. 2009; Luo and Gu 2009; Freitag et al. 2005).

\subsubsection{Live Oil Density}

Live oil density was measured at various times for each experiment as explained in Section 3.7. An average value for the live oil density was generated for each experiment. Average live oil density for various physical models is listed in Table 5.2, which shows small variations in the measured density values. The small variations allow us to assume constant live oil density, which is utilized in the simulation. A sensitivity study on the density supported this assumption, since the effect of the density variation on the dispersion values was found to be insignificant (Section 5.2.6). Moreover, introducing density as a function of solvent mass fraction would severely complicate our optimal control derivations and simulations, which already are so intensive. 
Table 5.2 Average live oil density $\left(\mathrm{g} / \mathrm{cm}^{3}\right)$ for various physical models

\begin{tabular}{l|c|c|c}
\hline Permeability & \multicolumn{3}{|c}{ Model height } \\
\hline \multirow{3}{*}{ BT4 (204 Darcy) } & $25 \mathrm{~cm}$ & $35 \mathrm{~cm}$ & $45 \mathrm{~cm}$ \\
\cline { 2 - 4 } BT5 (102 Darcy) & 0.830 & 0.828 & 0.819 \\
BT6 (51 Darcy) & 0.829 & 0.820 & 0.812 \\
\hline
\end{tabular}

The live oil density was calculated using ideal mixing law and the calculated values were compared with the average experimental measured ones listed in table 5.2. The maximum absolute error was $3 \%$.

\subsubsection{Live Oil Viscosity}

As solvent diffuses into heavy oil, viscosity of solvent-heavy oil system is drastically reduced. Viscosity reduction of the mixture would affect the mobility and the fluid flow mechanism of the process. A viscosity model is crucial in Vapex since it governs the movement of live oil in the reservoir, and thus has a direct bearing on oil production. The application of generic correlations of viscosity is not satisfactory since solvent-heavy oil and bitumen systems are not necessarily identical to each other. Only a system-specific correlation representing the true behaviour of the viscosity reduction during Vapex can assure the reliability of the mathematical model. Thus, the viscosity of propane-heavy oil system (live oil produced) was generated from the conducted experiments as explained in Section 3.6. Table 5.3 shows the experimentally measured live oil viscosity averaged for each experiment. The live oil viscosity is a strong function of propane mass fraction. It was reduced by five orders of magnitude and this would affect the mobility of the diluted oil. 
Table 5.3 Average live oil viscosity (cP) for various physical models

\begin{tabular}{l|c|c|c}
\hline Permeability & \multicolumn{3}{|c}{ Model height } \\
\hline & $25 \mathrm{~cm}$ & $35 \mathrm{~cm}$ & $45 \mathrm{~cm}$ \\
\cline { 2 - 4 } BT4 (204 Darcy) & 9.3 & 8.53 & 6.239 \\
BT5 (102 Darcy) & 6.497 & 4.4 & 3.5 \\
BT6 (51 Darcy) & 4.014 & 3.5 & 2.99 \\
\hline
\end{tabular}

Experimental live oil viscosity and the corresponding amount of propane dissolved in heavy oil data were best fitted to obtain the live oil viscosity concentration-dependent model. Figure 5.3 is showing the viscosity as a function of propane mass fraction described by the power law relationship. The empirical correlation for the propane-heavy oil system during the process at the operating temperature and pressure is:

$$
\mu=1.158 \omega^{-2} \quad 0.28 \leq \omega \leq 0.45
$$

with a high value of 0.982 for the $r^{2}$-coefficient of determination.

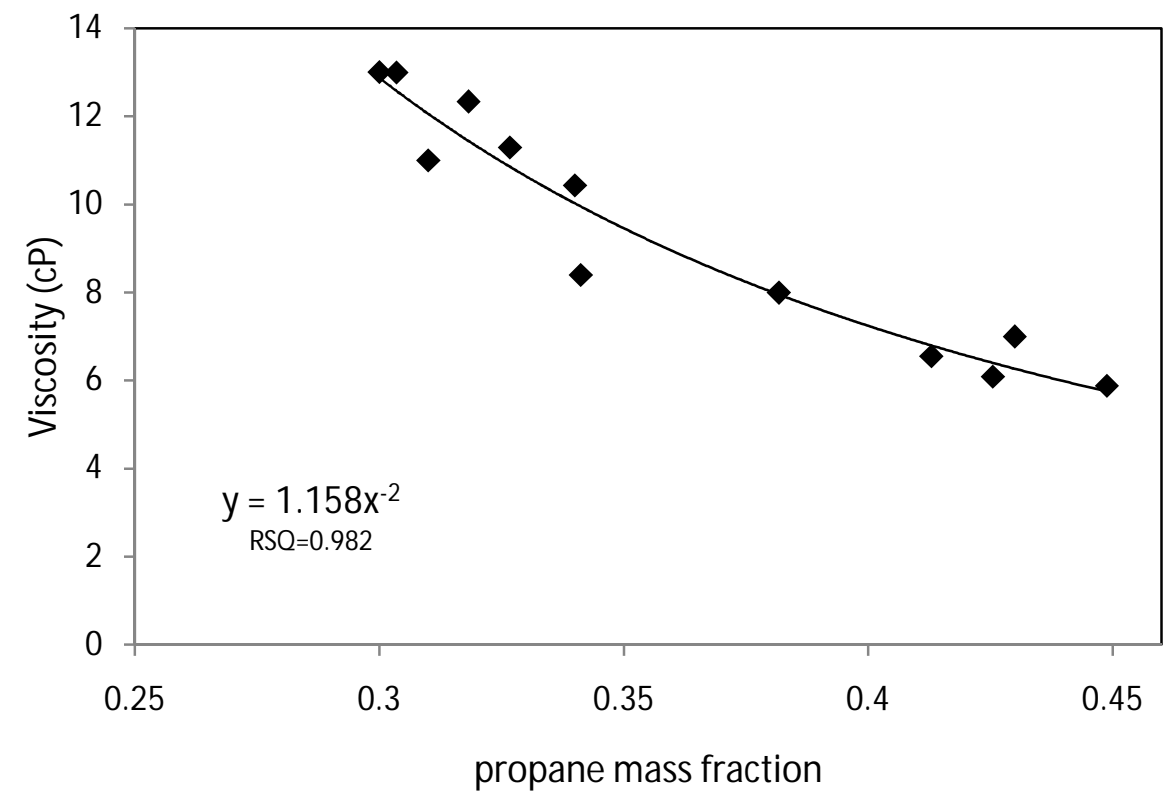

Figure 5.3 Live oil viscosity versus propane mass fraction 
The obtained live oil viscosity concentration-dependent model was utilized in the numerical simulation to determine the dispersion values, and it was extrapolated to a wider range of propane mass fraction $(\omega \leq 0.75)$. A justification for the extrapolation is found in Appendix $\mathrm{H}$.

As shown in Section 5.1.2.1, the amount of propane dissolved in heavy oil (mass fraction) is a function of the physical model permeability and drainage height, and the viscosity is a function of propane mass fraction. This implies a permeability and drainage height dependence of live oil viscosity. As shown in Table 5.3, the average live oil viscosity is reduced more as the packing permeability decreases and as the drainage height increases. Ramakrishnan (2003) reported similar dependency of the live oil viscosity with permeability in the Vapex experiments of rectangular physical models.

\subsubsection{Live Oil Production Rates}

At start of each experiment, the load cell recorded an increase in the weight up to a maximum value at a certain time instance. At this time instance, the oil started to drain and the weight of the physical model continued to decrease. This behaviour is due to the diffusion of the solvent into the bitumen, which increases the weight of the physical model up to a time instance at which the viscosity of the bitumen reduces to a threshold value. At this threshold value, the gravity forces overcome the capillary forces and the diluted oil starts to drain. The time instance when the physical model weight started to decrease and live oil to drain was considered the zero time for the Vapex experiments.

The cumulative live oil produced as a function of time for Vapex experiments are shown in Figure 5.4 for glass beads packs permeabilities of 204, 102, and $51 \mathrm{D}$ in $45 \mathrm{~cm}$ model for 180

minutes. In the figure, the live oil production rates decrease when lower glass beads permeability is used. 


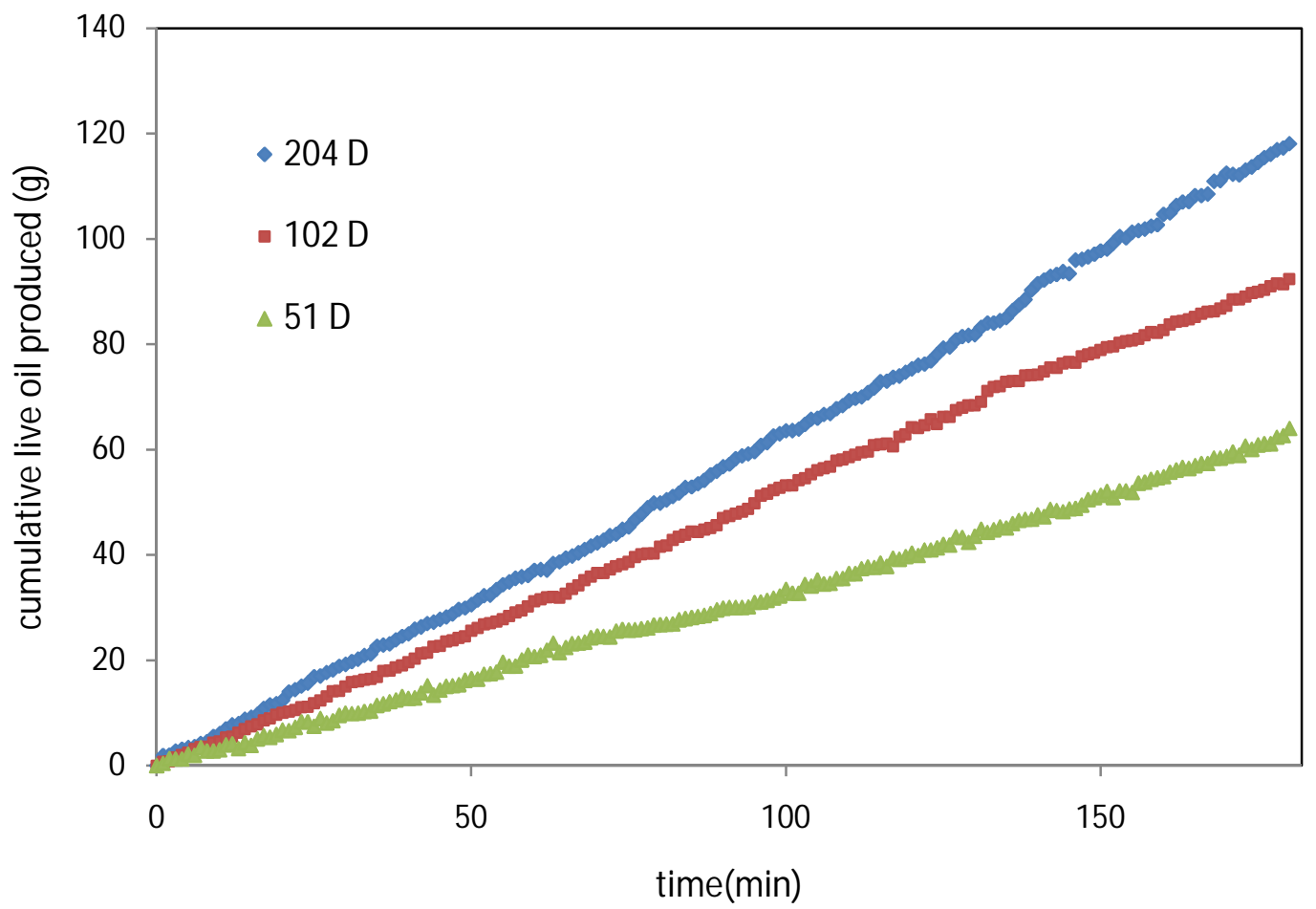

Figure 5.4 Cumulative live oil production versus time $(45 \mathrm{~cm})$

Figure 5.5 shows a comparison of the live oil production rates for physical models of 25,35 , and $45 \mathrm{~cm}$ drainage height with $204 \mathrm{D}$ permeability for 180 minutes. As the drainage height increases, higher production rates observed. The cumulative oil production curves for other different packing permeability and model heights used in the Vapex experiments are shown in Appendix F. 


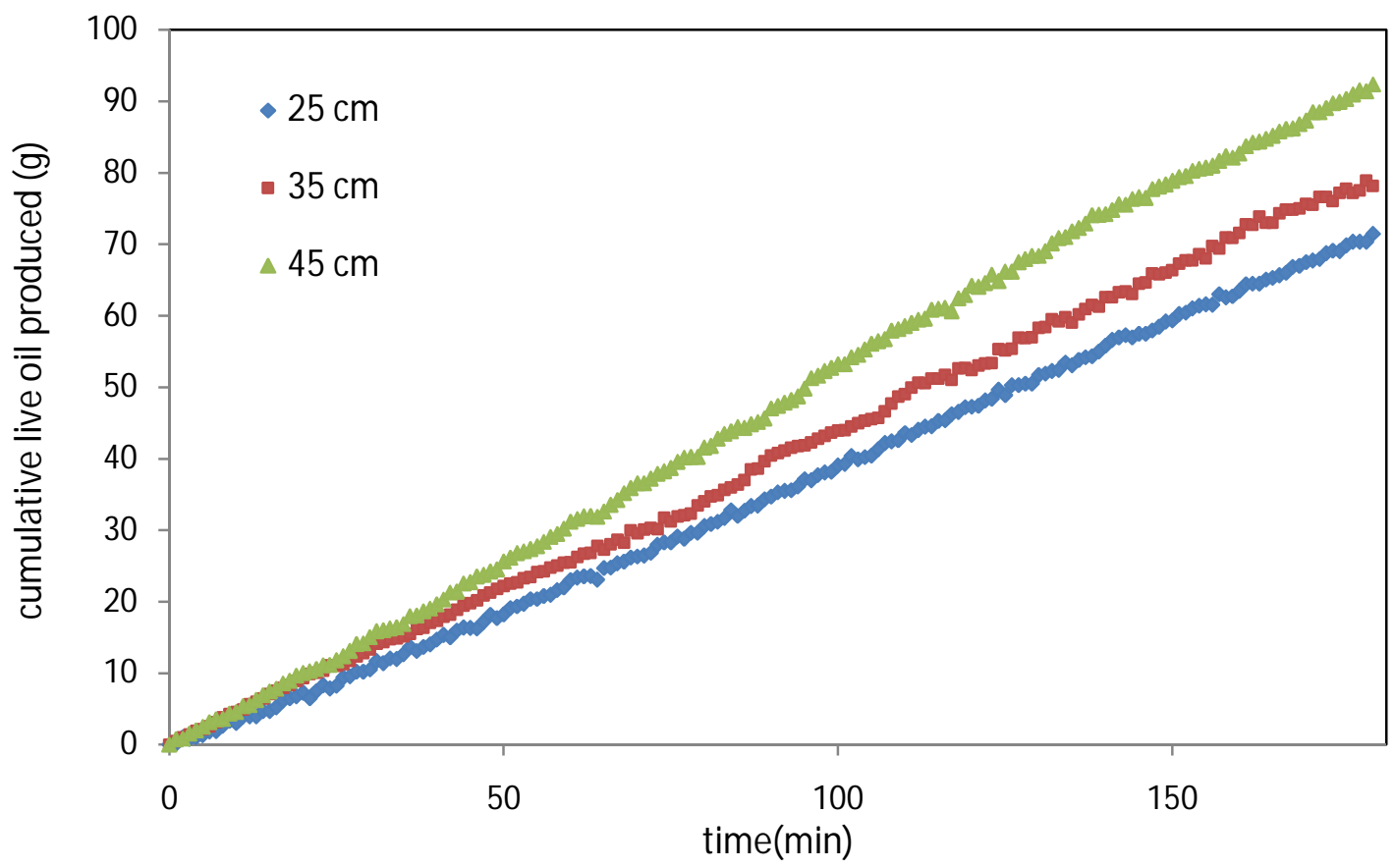

Figure 5.5 Cumulative live oil production versus time (204 D)

Applying linear regression on the approximately straight lines over a large portion of the cumulative oil production curves to obtain the steady live oil drainage rates. The live oil production rates are listed in Table 5.4.

Table 5.4 Experimental live oil production rates $(\mathrm{g} / \mathrm{min})$ for various packing permeability and drainage heights

\begin{tabular}{l|c|c|c}
\hline Permeability & \multicolumn{3}{|c}{ Model height } \\
\hline \multirow{3}{*}{ BT4 (204 Darcy) } & $25 \mathrm{~cm}$ & $35 \mathrm{~cm}$ & $45 \mathrm{~cm}$ \\
\cline { 2 - 4 } BT5 (102 Darcy) & 0.491 & 0.573 & 0.641 \\
BT6 (51 Darcy) & 0.392 & 0.442 & 0.522 \\
\hline
\end{tabular}


To gain deeper understanding of the Vapex mechanisms, the average drainage velocity is calculated and compared for the various physical models used in the Vapex experiments. The average drainage velocity is calculated based on the Darcy's law given by Equation (4.4), employing the live oil properties measured experimentally. The average calculated drainage velocities for various physical models are shown in Table 5.5. The average velocities values indicate the permeability and drainage height functionalities. The main reason is primarily that velocity is a function of live oil viscosity and as shown in Section 5.1.2.3, live oil viscosity is permeability and drainage height dependent.

Table 5.5 Calculated average drainage live oil velocity $(\mathrm{m} / \mathrm{s})$ for various packing

\begin{tabular}{l|c|c|c}
\hline Permeability & \multicolumn{3}{|c}{ Model height } \\
\hline & $25 \mathrm{~cm}$ & $35 \mathrm{~cm}$ & $45 \mathrm{~cm}$ \\
\cline { 2 - 4 } BT4 (204 Darcy) & $1.76 \times 10^{-4}$ & $1.92 \times 10^{-4}$ & $2.59 \times 10^{-4}$ \\
BT5 (102 Darcy) & $1.26 \times 10^{-4}$ & $1.84 \times 10^{-4}$ & $2.29 \times 10^{-4}$ \\
BT6 (51 Darcy) & $1.01 \times 10^{-4}$ & $1.15 \times 10^{-4}$ & $1.34 \times 10^{-4}$ \\
\hline
\end{tabular}

Although the physical model with lower permeability produces lower live oil viscosity with higher propane mass fraction, the average drainage velocity is decreasing. This is justified by the smaller pore throats sizes in the porous medium result when the pack permeability is decreasing, thus the live oil can flow less easily through the porous medium and the live oil is produced slower. With a higher drainage height, lower live oil viscosity results with higher propane mass fraction and the average drainage velocity is increasing.

Re-examining the stabilized live oil production rates for the three drainage heights in Figure 5.5, the experimental live oil production rate increases with the model drainage height. The production rate for $35 \mathrm{~cm}$ drainage height is $14 \%$ higher than that for $25 \mathrm{~cm}$ drainage height. Moreover, the production rate increases by $11 \%$ when the drainage height increases from 35 to $45 \mathrm{~cm}$. This can be explained by the increase of the amount of propane dissolved in the heavy 
oil, which has a great bearing on the live oil viscosity. As the live oil viscosity reduces, the average drainage velocity becomes higher. Thus, the live oil production rate in Vapex is dependent on the model drainage height. Das and Butler (1998) and Yazdani and Maini (2005) reported similar dependency of live oil production rates with drainage height in the Vapex experiments.

\subsection{Numerical Results and Discussion}

In this section, the results of an extensive simulation study of the Vapex process will be described and discussed. The main objective of the numerical simulation work was to get the best possible matching between the model-predicted and the experimental Vapex production rates by adjusting the dispersion function as the matching parameter. As mentioned earlier, experiments were conducted using laboratory scale physical models at $21^{\circ} \mathrm{C}$ and $0.790 \mathrm{MPa}$ pressure. Under these conditions, the cumulative production mass of live oil was measured and averaged every 1 minute. To increase the confidence in the results of numerical simulation and the inferred matching dispersion function, the rest of the parameters that are involved in the simulation model were measured experimentally such as live oil viscosity, density, and propane mass fraction. The characteristics of the porous medium were also measured experimentally as explained in Chapter 3.

\subsubsection{Algorithm Execution}

The state equations [Equations (4.59) and (4.60)] are solved simultaneously to obtain the calculated cumulative mass of produced live oil at different time instances employing the developed computational algorithm. The concentration-dependent dispersion coefficient of propane, $D(\omega)$, and propane-heavy oil interface mass fraction, $\omega_{\text {int }}$, are the two unknown parameters in the state equations. Therefore, propane dispersion function will be determined simultaneously with propane-heavy oil mass fraction $\left(\omega_{\text {int }}\right)$ at the interface. The approach is to

choose various values of $\left(\omega_{\text {int }}\right)$ and $D(\omega)$ in the range of $0.6-1$ and $10^{-7}-1.5 \times 10^{-5} \mathrm{~m}^{2} / \mathrm{s}$ respectively. 
Applying initial guesses of the uniform propane dispersion function and propane-heavy oil interface mass fraction, the non-dimensionalized discretized state equations [Equations (4.63)(4.71), Equation (4.74), and Equation (4.75)] are solved simultaneously to yield propane mass fraction in the heavy oil in porous medium, $\omega(t, r, z)$, and the height of bitumen, $Z(t, r)$, in the physical model. In Equations (4.74) and (4.75), Darcy's velocity at the bottom of the physical model is calculated as the average velocity over each grid size. The velocity profile as a function of the radius of the physical model is shown in Appendix I.

Knowing propane mass fraction in the heavy oil, $\omega(t, r, z)$, and the height of bitumen, $Z(t, r)$, in the physical model, the mass of oil produced, i.e. $m_{c}(t)$ [Equation (4.95)], at the experimental time instants can be calculated. This step leads to the calculation of the objective function given by Equation (4.14). If the discrepancy between the experimental and simulated mass of live oil produced is not acceptable, then the dispersion function should be improved to minimize the objective function.

To improve the dispersion function $D(\omega)$, the gradient correction with respect to $D$ is calculated. Using the saved values of $(\omega)$ and $(Z)$ at the experimental time instants, the nondimensionalized discretized costate equations [Equation (4.80)-(4.86)] and Equation (4.87) is solved simultaneously to yield the values of adjoint variables $\lambda(t, r, z)$ and $\gamma(t, r)$. Having the values of the adjoint variables, the gradient correction with respect to $D$ is calculated. Finite difference approximation is used to calculate the gradient correction with respect to $D$ using Equations (4.88)-(4.94). The gradient corrections in dispersion are averaged over the whole experimental run time. The values calculated to improve the dispersion functional are used in the optimization algorithm, BFGS, explained in Chapter 4.

The BFGS method is used to iteratively improve $D(\omega)$ and minimize the objective function. The BFGS algorithm is programmed to terminate when the change in the gradient correction becomes very small as less than or equal to $10^{-6}$. During the computations, cubic splines are used to interpolate $D(\omega)$ as well as its first and second derivatives with respect to $\omega ; m_{\mathrm{c}}(t)$ and $m_{\mathrm{e}}(t) ; \omega$ and $Z$; and the variational derivative $J_{\mathrm{D}}$ at each specified solvent mass fraction. 
Table 5.6 lists the parameters used in the simulation of the mathematical model for physical model of $204 \mathrm{D}$ permeability and $25 \mathrm{~cm}$ drainage height.

Table 5.6 Simulation model parameters $(204 \mathrm{D}$ and $25 \mathrm{~cm})$

\begin{tabular}{|l|l|}
\hline Parameter & Value \\
\hline$\varphi$ & 0.38 \\
\hline $\mathrm{K}_{\mathrm{r}}$ & 1 \\
\hline $\mathrm{K}, \mathrm{cm}^{2}$ & $2.013 \times 10^{-6}$ \\
\hline $\mathrm{R}, \mathrm{cm}$ & 3 \\
\hline $\mathrm{Z}_{0}, \mathrm{~cm}$ & 25 \\
\hline$\mu_{0}, \mathrm{~g} / \mathrm{cm} \cdot \min$ & 0.695 \\
\hline$\rho, \mathrm{g} / \mathrm{cm}^{3}$ & 0.821 \\
\hline
\end{tabular}

Since the drainage flow rate remained the same up to 180 minutes and the physical model is symmetrical, there should be no difference if a shorter time of simulation is selected. In our numerical simulations, we found that the results changed negligibly using the data extended beyond 60 minutes. However, it took us weeks to get the results due to optimization. A comparison between the simulation results for both 60 minutes and 120 minutes simulation time was obtained. The average absolute errors in dispersion coefficients values were $0.101 \times 10^{-5}$ and $0.133 \times 10^{-5} \mathrm{~m}^{2} / \mathrm{s}$ for the two cases investigated. Results to support this claim are shown in Appendix G.

\subsubsection{Propane-Heavy Oil Interface Mass Fraction}

As explained in the previous section, several simulations were performed at various values of $\omega_{\text {int }}$ and $D(\omega)$. Table 5.7 shows the objective functions for various interface propane mass fractions and various uniform dispersion coefficients used in the simulation. The objective functions shown in the table are less than $4 \times 10^{2}\left(\mathrm{~g}^{2} \cdot \mathrm{min}\right)$, and surround the minimum objective function. 
Table 5.7 Objective functions at various interface mass fractions of propane and initial uniform dispersion values $(25 \mathrm{~cm}, 204 \mathrm{D})$

\begin{tabular}{|c|c|c|c|c|c|c|c|c|}
\hline $\begin{array}{c}D(\omega) \times 10^{5} \\
\left(\mathrm{~m}^{2} / \mathrm{s}\right)\end{array}$ & \multicolumn{7}{|c|}{ Interface mass fraction of propane } \\
\cline { 2 - 9 } & 0.73 & 0.74 & 0.75 & $\mathbf{0 . 7 6}$ & 0.78 & 0.8 & 0.82 & 0.83 \\
\hline 0.32 & 84.3 & 57.2 & 37.9 & 36.4 & 85.76 & 152.1 & 156.7 & 161.87 \\
\hline 0.39 & 93.7 & 52.98 & 34.25 & 33.44 & 84.56 & 153.8 & 165.6 & 177.1 \\
\hline $\mathbf{0 . 5}$ & 97.06 & 59.8 & 36.9 & $\mathbf{3 2 . 5}$ & 83.2 & 154 & 188.9 & 201.05 \\
\hline 0.667 & 118.3 & 65.1 & 40.2 & 35.4 & 84.1 & 161.7 & 176.9 & 210.2 \\
\hline 0.833 & 125.3 & 77.3 & 45.44 & 39.8 & 88.8 & 167.04 & 238.5 & 256.5 \\
\hline
\end{tabular}

Figure 5.6 shows the variation of the objective function with respect to various interface propane mass fractions with dispersion coefficient of $0.5 \times 10^{-5} \mathrm{~m}^{2} / \mathrm{s}$ used in the simulation. It can be analyzed from Figure 5.6 that optimum objective function found was 32.5 with corresponding optimal value of $\omega_{\text {int }}=0.76$. Further calculations with $\omega_{\text {int }}>0.83$, and $D(\omega)>0.833 \mathrm{~m}^{2} / \mathrm{s} \mathrm{did} \mathrm{not}$ result objective functions lower than its optimal value of 32.5. The same value of $\omega_{\text {int }}$ was used to determine the dispersion of propane as a function of its concentration in heavy oil for the various physical models utilized in the experiments since the operating conditions are similar. 


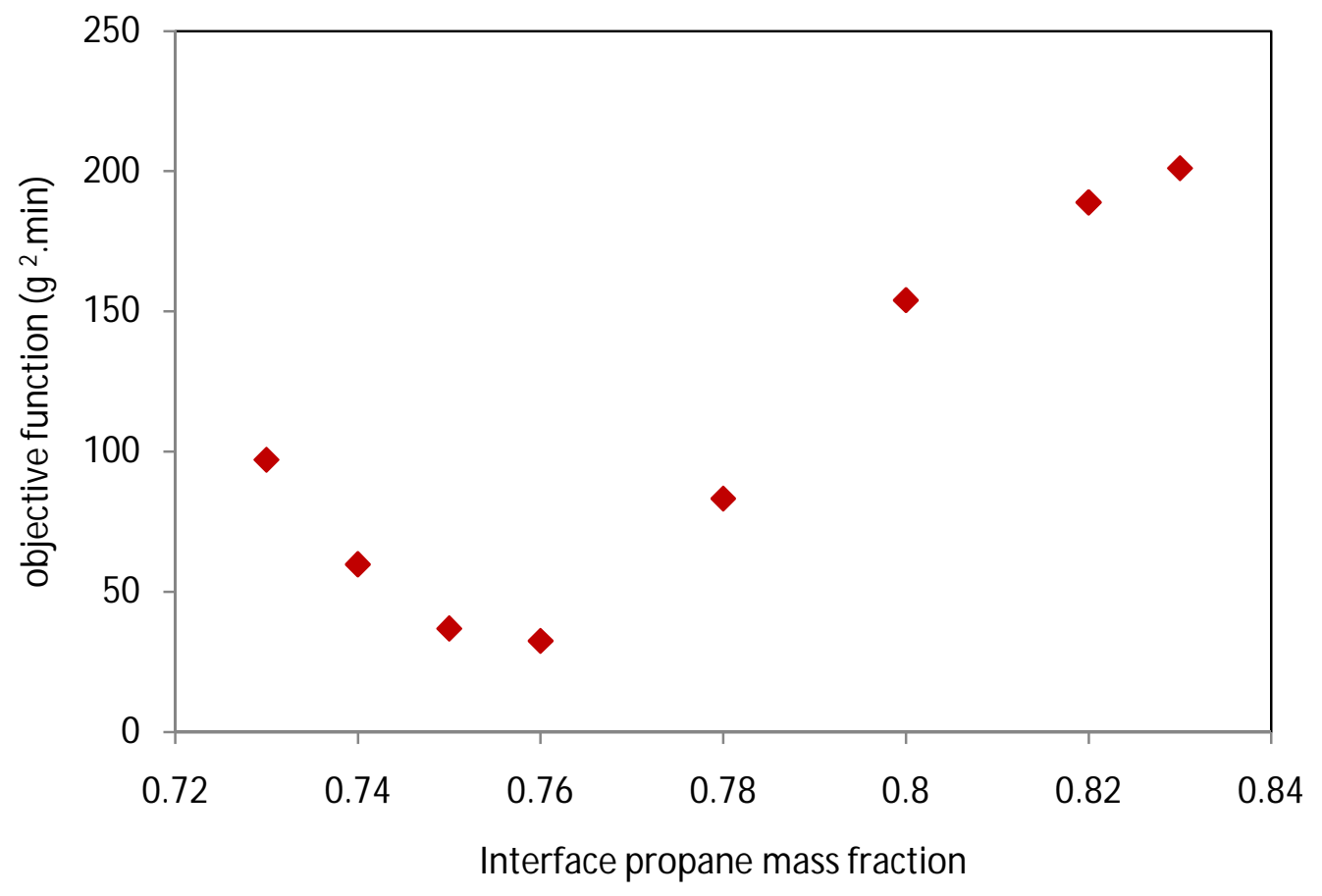

Figure 5.6 Objective functions versus solvent interface mass fraction for initial dispersion of $0.5 \times 10^{-5} \mathrm{~m}^{2} / \mathrm{s}$.

\subsubsection{Determination of Concentration-Dependent Dispersion Coefficient}

The simulation results for propane-heavy oil system with glass beads of 204 Darcy and drainage height of $25 \mathrm{~cm}$, at $\omega_{\text {int }}=0.76$ are presented. The simulation resulted in an iterative reduction of the objective function accompanied by a corresponding improvement in $D(\omega)$. The objective function decreased monotonically to the minimum as shown in Figure 5.7. The change was significant at the beginning, but the rate of improvement slowed down at final iterations. The minimal objective function is that point when the gradient correction given by Equation (4.55) tends to zero. No further improvement is possible then. The final optimal function $D(\omega)$ was obtained in 29 iterations after which no further improvement was observed. 


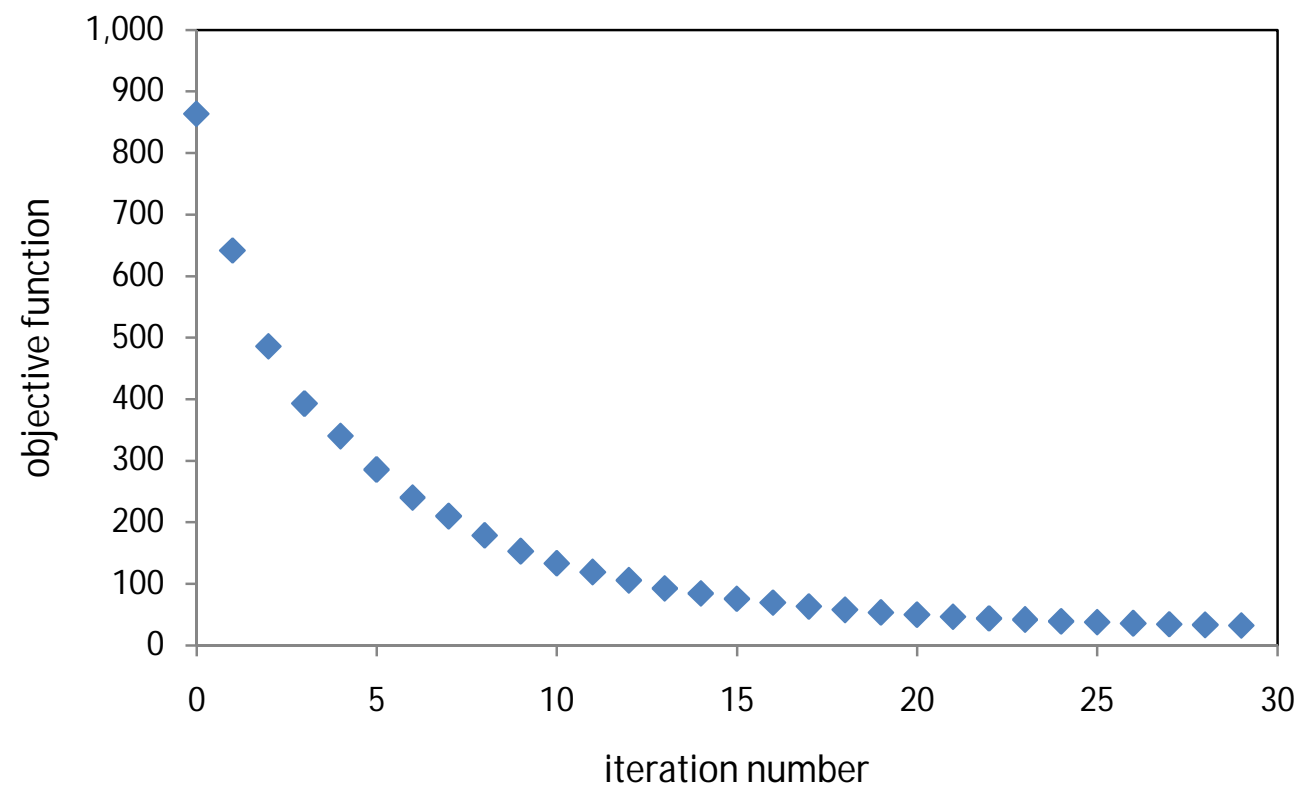

Figure 5.7 Objective function versus iteration number (204 D and $25 \mathrm{~cm})$.

The initial and optimal $D(\omega)$ are plotted in Figure 5.8. It shows that the optimally determined $D(\omega)$ rises to a maximum value, and then drops toward the end. The maximum value of propane dispersion is $4.048 \times 10^{-5} \mathrm{~m}^{2} / \mathrm{s}$ at the corresponding propane mass fraction of 0.336 . Although this figure presents all the values up to $\omega=0.75$, the practical range of this figure for Vapex applications is determined by the solubility of propane at operational conditions. 


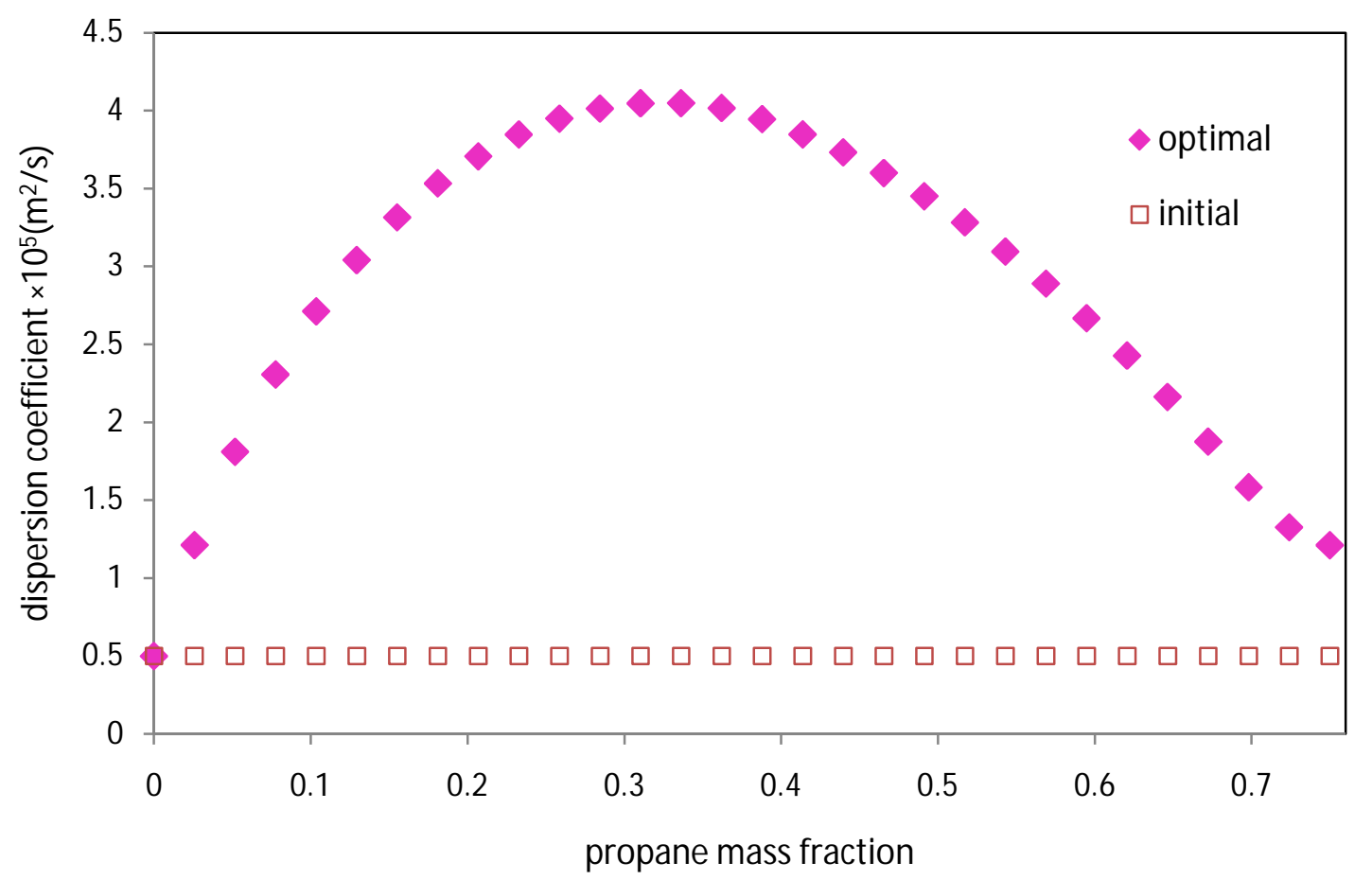

Figure 5.8 Dispersion coefficient function of propane in heavy oil $(204 \mathrm{D}, 25 \mathrm{~cm})$

The concentration dependence of dispersion coefficient is expected since the phenomenon of diffusion embodied in dispersion is strongly affected by solvent concentration. Moreover, mechanical (convective) dispersion as a major component of dispersion is affected by velocity variation, which is a function of solvent concentration. The maximum dispersion coefficient is 8 times the minimum, thus indicating the strong concentration dependence of propane dispersion.

The maximum in the concentration-dependent dispersion function could be explained as follows. Initially when higher concentration gradients are present in the heavy oil, the diffusion, and the convective dispersion of solvent molecules is higher. It subsides later on with a gradual reduction in the concentration gradients as more and more solvent's molecules penetrate the medium because of dispersion. When this happens, the motion of solvent molecules is restricted by their own abundance, thus decreasing the overall solvent dispersive flux. When the solvent diffusion 
flux and the convective flux are at their maximum, the dispersion coefficient value gains the highest value. Thus, at some intermediate stage, the dispersion coefficient is at its maximum.

Figure 5.9 compares the experimental live oil production to the calculated one with the optimally determined propane dispersion. The calculated production follows experimental production very closely during the operation time of about 60 minutes. It implies that the gradient corrections in dispersion achieved the desired minimum.

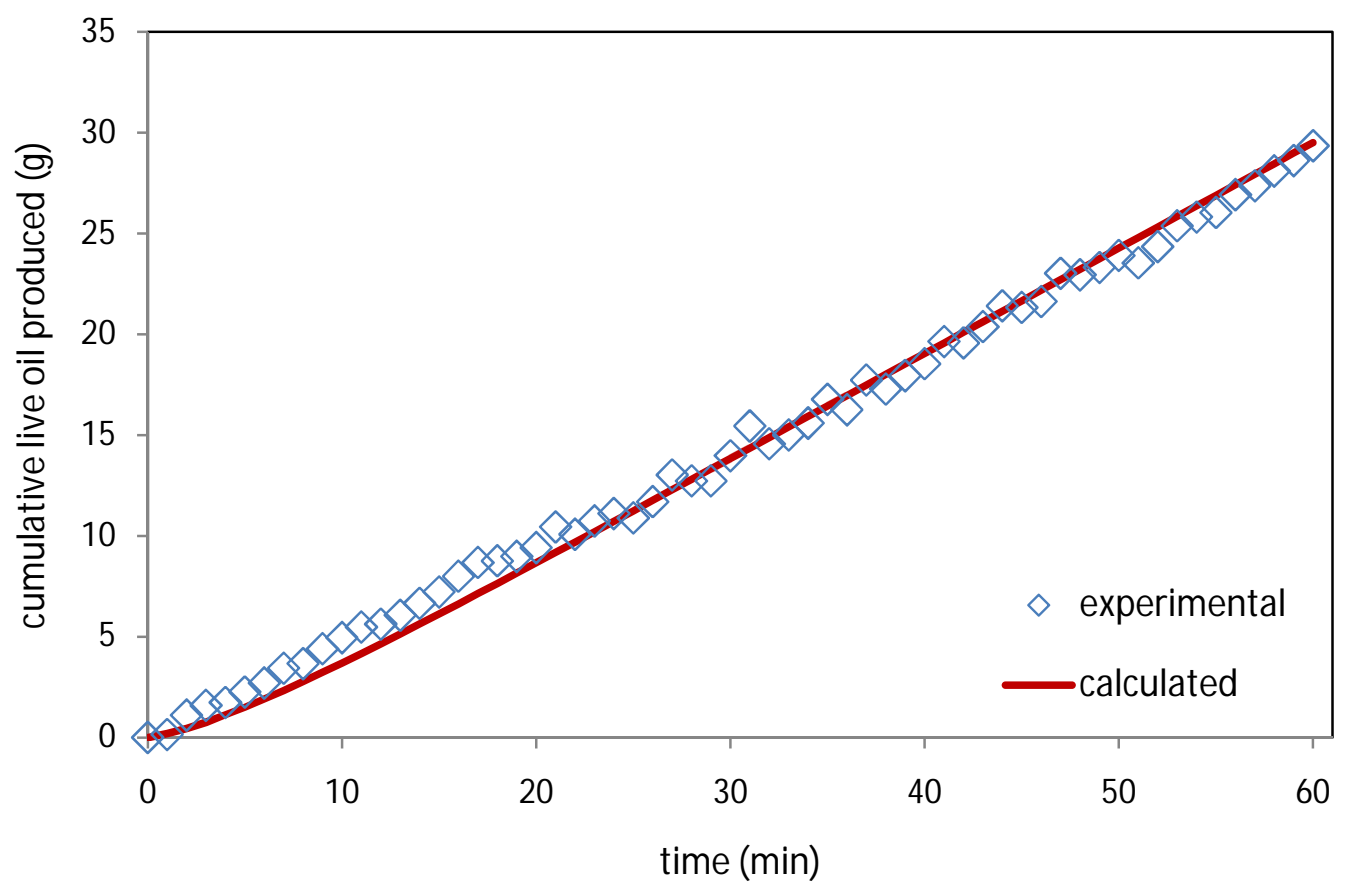

Figure 5.9 Experimental and calculated mass of live oil produced with time $(204 \mathrm{D}, 25 \mathrm{~cm})$

It has to be noted that we did not specify, or constrain the form of concentration-dependent dispersion function, but enabled its natural and realistic determination. In comparison to the molecular diffusion coefficient of propane in heavy oil (Tharanisivan et al., 2006; Das and Butler, 1996; Yang and $\mathrm{Gu}, 2006$ ) the average dispersion coefficient obtained in this work is up to four orders of magnitude higher, and underscores the strong effect of convective dispersion and other phenomena that augment mixing. 
The above outcome has a direct bearing on the optimal operations of Vapex implementations. For example, to maximize solvent uptake by the reservoir and oil production as a consequence, solvent injection rates should be such that the average solvent mass fraction in the reservoir (at $21^{\circ} \mathrm{C}$ and $\left.0.790 \mathrm{MPa}\right)$ is close to the optimal solvent mass fraction $(0.336)$ corresponding to the peak value of dispersion $\left(4.048 \times 10^{-5} \mathrm{~m}^{2} / \mathrm{s}\right.$; about twice the average value of dispersion). Hence, in addition to enabling more accurate reservoir simulations, the concentration-dependent dispersion function provides insights into optimizing Vapex operations as well.

\subsubsection{Model Validation}

The numerical model was validated first to ensure consistent numerical results before being used to predict the dispersion function using various physical models. Using the optimal propane interface mass fraction $\omega_{\text {int }}=0.76$ and the optimal dispersion function determined for $204 \mathrm{D}$ permeability and $25 \mathrm{~cm}$ drainage height, the simulated production rates were compared with the experimental ones obtained from another data set of an experiment conducted for the same physical model at the same operating conditions. Figure 5.10 shows that the experimental and calculated production rates agree very well when the optimal dispersion function determined previously was used.

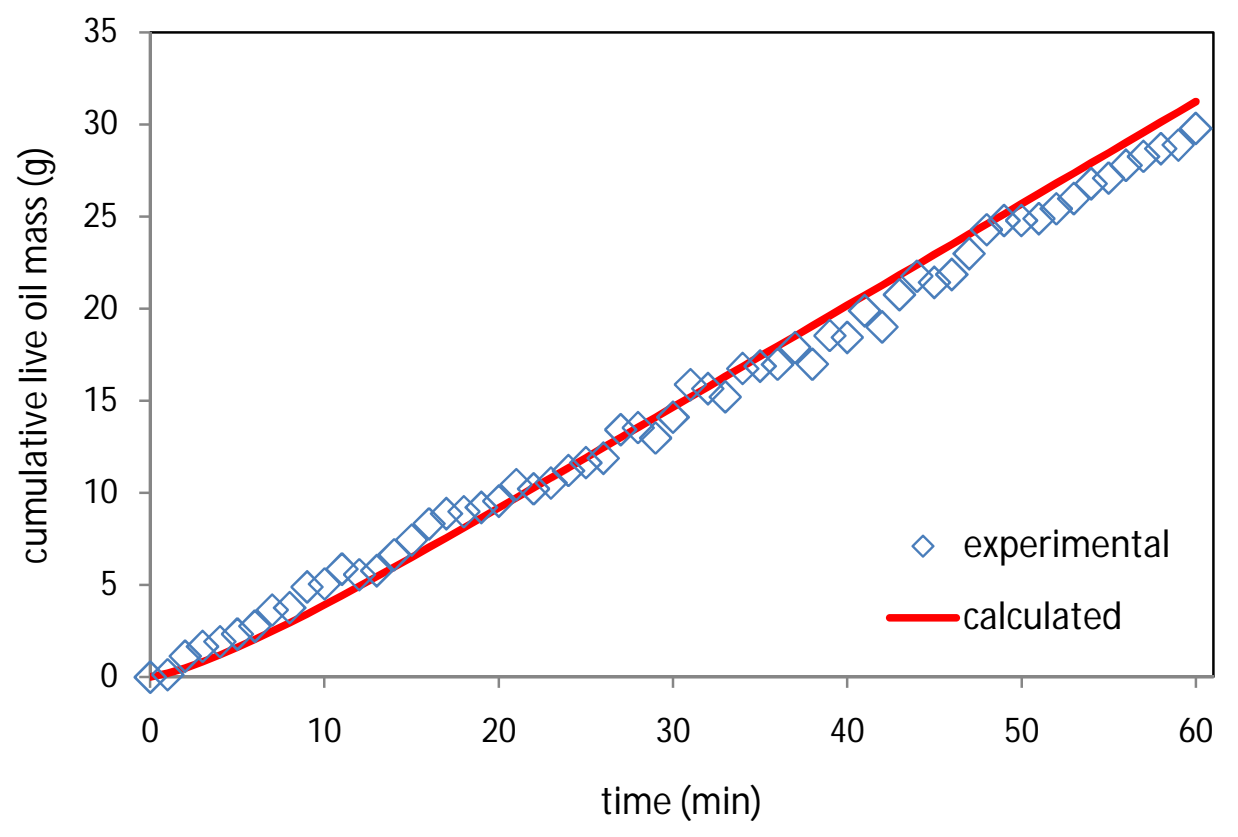

Figure 5.10 Model validation (204 D and $25 \mathrm{~cm})$ 


\subsubsection{Calculated Change of Bitumen Height in the Porous Medium}

For the optimal values of $\omega_{\text {int }}$ and $D(\omega)$, Figure 5.11 shows the variation of height of the bitumen in the 204 Darcy packing with $25 \mathrm{~cm}$ drainage height at different times. The height is observed to reduce as well the radius with time. The figure shows that the simulated bitumen height moves in a relatively stable manner, which results a constant Vapex interface shape.

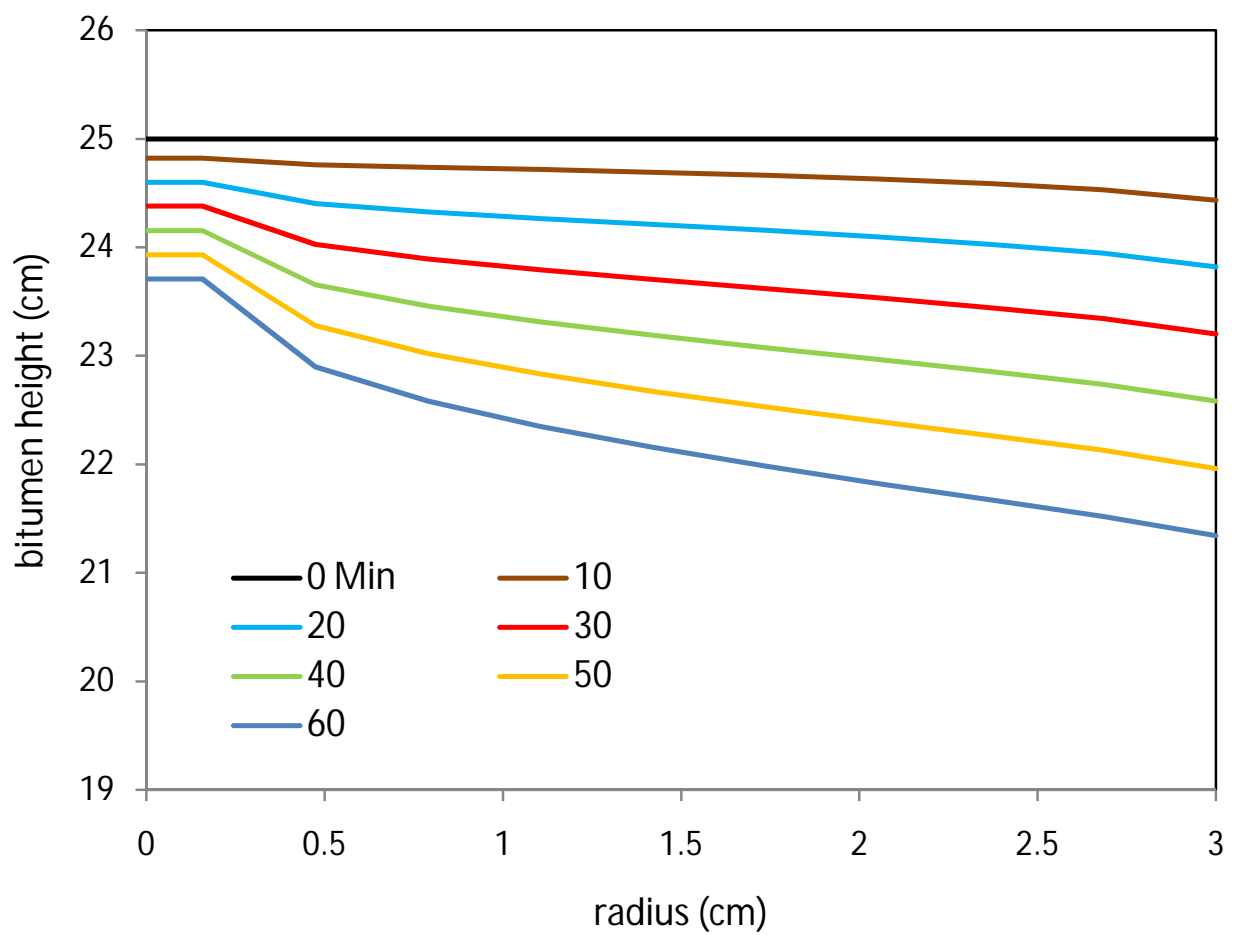

Figure 5.11 The change in bitumen height with time (204 D, $25 \mathrm{~cm})$.

\subsubsection{Sensitivity Analyses of the Model Parameters}

A sensitivity analysis of the model parameters on the optimal propane dispersion coefficient values was undertaken by varying live oil density $(\rho)$, grids number, live oil viscosity coefficient $\left(\mu_{o}\right)$, porous medium permeability and porosity, initial dispersion $D(\omega)$, and propane interface mass fraction $\left(\omega_{\text {int }}\right)$. The results of the sensitivity analyses were compared with 
the base average dispersion value for $204 \mathrm{D}$ permeability and $25 \mathrm{~cm}$ drainage height. Table 5.8 shows the average change in dispersion values. For a $\pm 2 \%$ variation in live oil density $(\rho)$, the change in average dispersion values is about $4 \%$ of the base value. For a $\pm 2 \%$ variation in the viscosity coefficient $\left(\mu_{o}\right)$, the change in average dispersion values is about $0.16 \%$ of the base value. The change in average dispersion values is about $9 \%$ of the base value when changing the number of the grids in the radial direction. However, the change in average dispersion values is about $1 \%$ of the base value when changing the number of the grids in the vertical direction.

For a $\pm 2 \%$ variation in the porous medium porosity and permeability, the change in average dispersion values is about $2 \%$, and $3 \%$ of the base value respectively. For a $\pm 2 \%$ variation in the initial dispersion value, the change in average dispersion values is about $0.2 \%$ of the base value. For a $\pm 2 \%$ variation in propane interface mass fraction, $\left(\omega_{\text {int }}\right)$, the change in average dispersion values is about $21 \%$ of the base value.

Table 5.8 Change in average dispersion values

\begin{tabular}{|c|c|c|}
\hline parameter & Variation in parameter & average change in dispersion $\mathbf{\times} \mathbf{1 0}^{\mathbf{5}} \mathbf{( \mathbf { m } ^ { \mathbf { 2 } } / \mathbf { s } )}$ \\
\hline \multirow{2}{*}{ Live oil density $(\rho)$} & $+2 \%$ & -0.123 \\
& $-2 \%$ & 0.112 \\
\hline \multirow{2}{*}{ Viscosity coefficient $\left(\mu_{o}\right), \mathrm{CP}$} & $+2 \%$ & 0.00929 \\
& $-2 \%$ & -0.00034 \\
\hline \multirow{3}{*}{ Grid number } & $N_{r}=25, N_{z}=10$ & 0.239 \\
& $N_{r}=17, N_{z}=10$ & -0.282 \\
& $N_{r}=20, N_{z}=12$ & 0.048 \\
& $N_{r}=20, N_{z}=8$ & -0.011 \\
\hline
\end{tabular}

Dispersion values are not sensitive to variations in live oil density, viscosity coefficient, permeabiltity, porosity, initial guess of dispersion. The dispersion values are very sensitive to propane interface mass fraction value since the driving force in Vapex process is the difference 
between propane-bitumen interface concentration $\left(\omega_{\text {int }}\right)$ and propane concentration in the bitumen $(\omega)$.

\subsection{Permeability Effect on Concentration-Dependent Dispersion Coefficient}

The results in Section 5.1 have been used to determine the concentration-dependent dispersion coefficient of propane in heavy oil using a specific physical model drainage height packed with one size of the three different glass beads sizes (204 D, 102 D, and 51 D) each run. Using the optimal control algorithm (Section 4.3.5), dispersion coefficient versus propane mass fraction results were obtained for the three medium permeabilities at $21^{\circ} \mathrm{C}$ and at $0.790 \mathrm{MPa}$ pressure (Abukhalifeh et al., 2010). The application of the algorithm resulted in an iterative reduction of the objective functions accompanied by a corresponding improvement in $D(\omega)$. The objective functions values decrease monotonically to the minimum as shown in Figure 5.12 for 204, 102, and $51 \mathrm{D}$ permeabilities with $35 \mathrm{~cm}$ drainage height physical model. The final optimal function $D(\omega)$ was obtained after which no further improvement was observed.

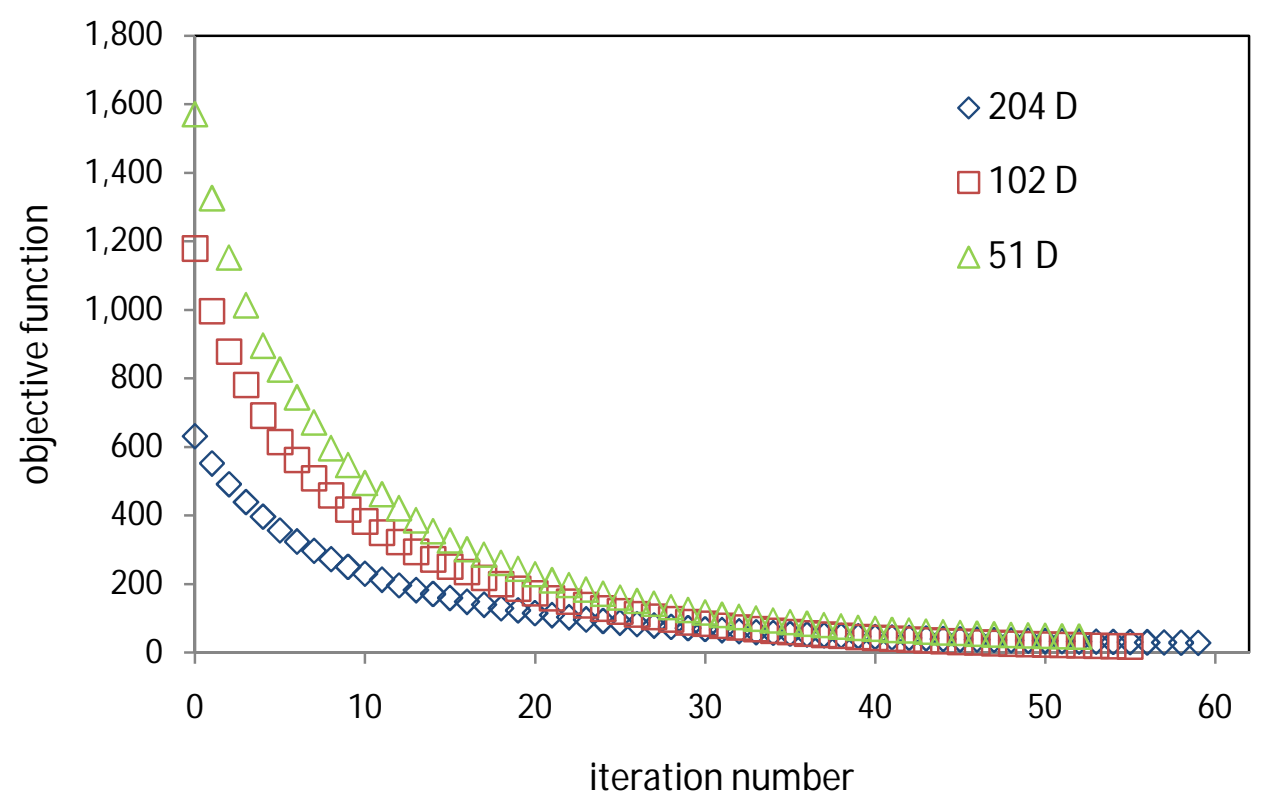

Figure 5.12 objective functions versus number of iteration for $35 \mathrm{~cm}$ drainage height model 
The optimal functions $D(\omega)$ obtained are plotted in Figure 5.13. As shown in the figure, the dispersion functions of propane for the three mediums with different permeabilities develop individually with a similar trend. Plots in Figure 5.13 show that the final optimally determined $D(\omega)$ rises to a maximum value, and then drops where propane mass fraction is higher.

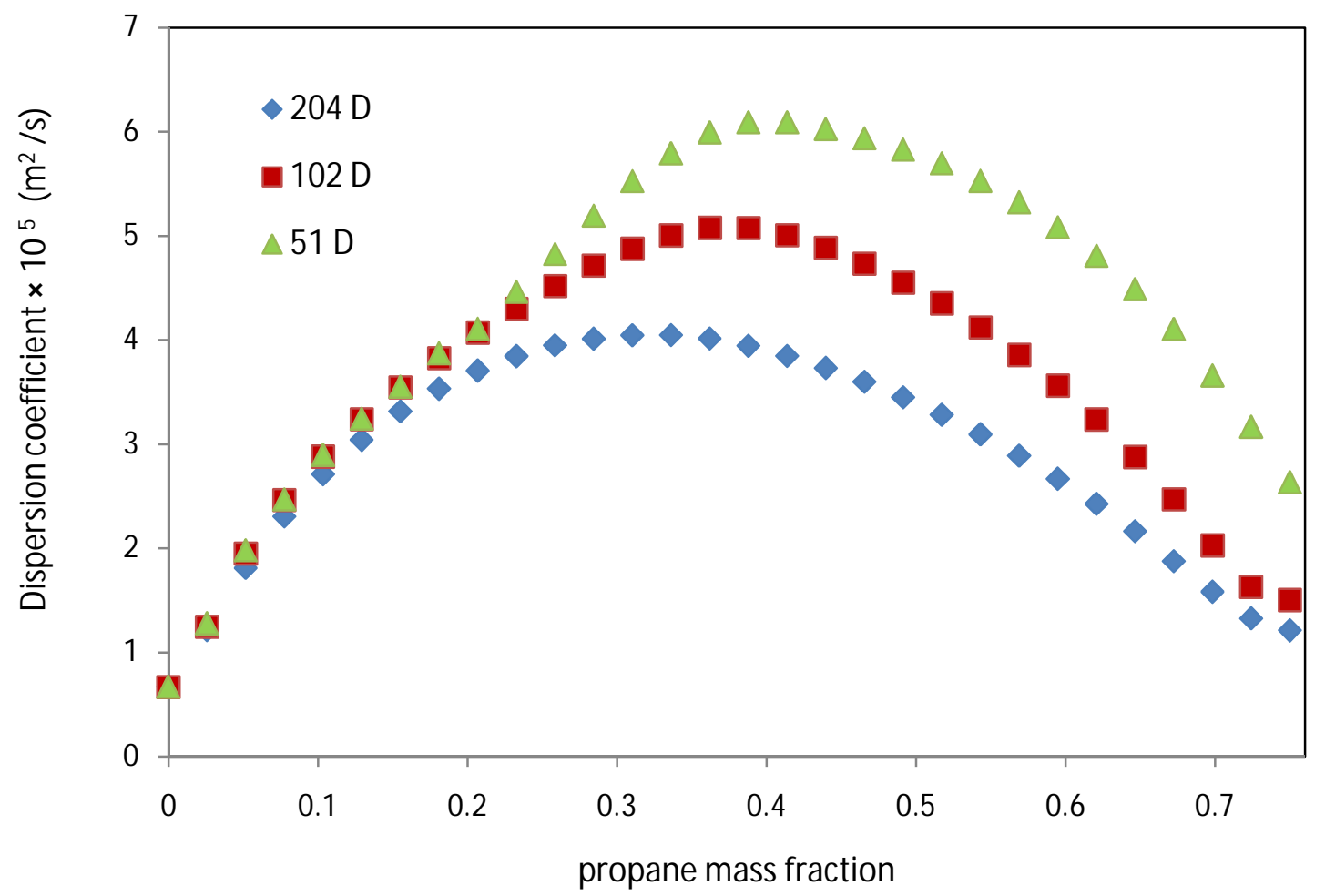

Figure 5.13 Dispersion coefficients of propane in heavy oil with three medium permeabilities $(25 \mathrm{~cm}$ drainage height)

Following the aforementioned optimal control algorithm, the optimal dispersion functions for the three packs permeability with a model height of $35 \mathrm{~cm}$ and $45 \mathrm{~cm}$ respectively were determined as shown in Figures 5.14 and 5.15. A similar trend is observed for propane dispersion function with its mass fraction. It is noteworthy that dispersion coefficient of propane in heavy oil is not a single value but it is a function of concentration. This function is not forced to follow any preassumed form, but it is allowed to develop gradually. 


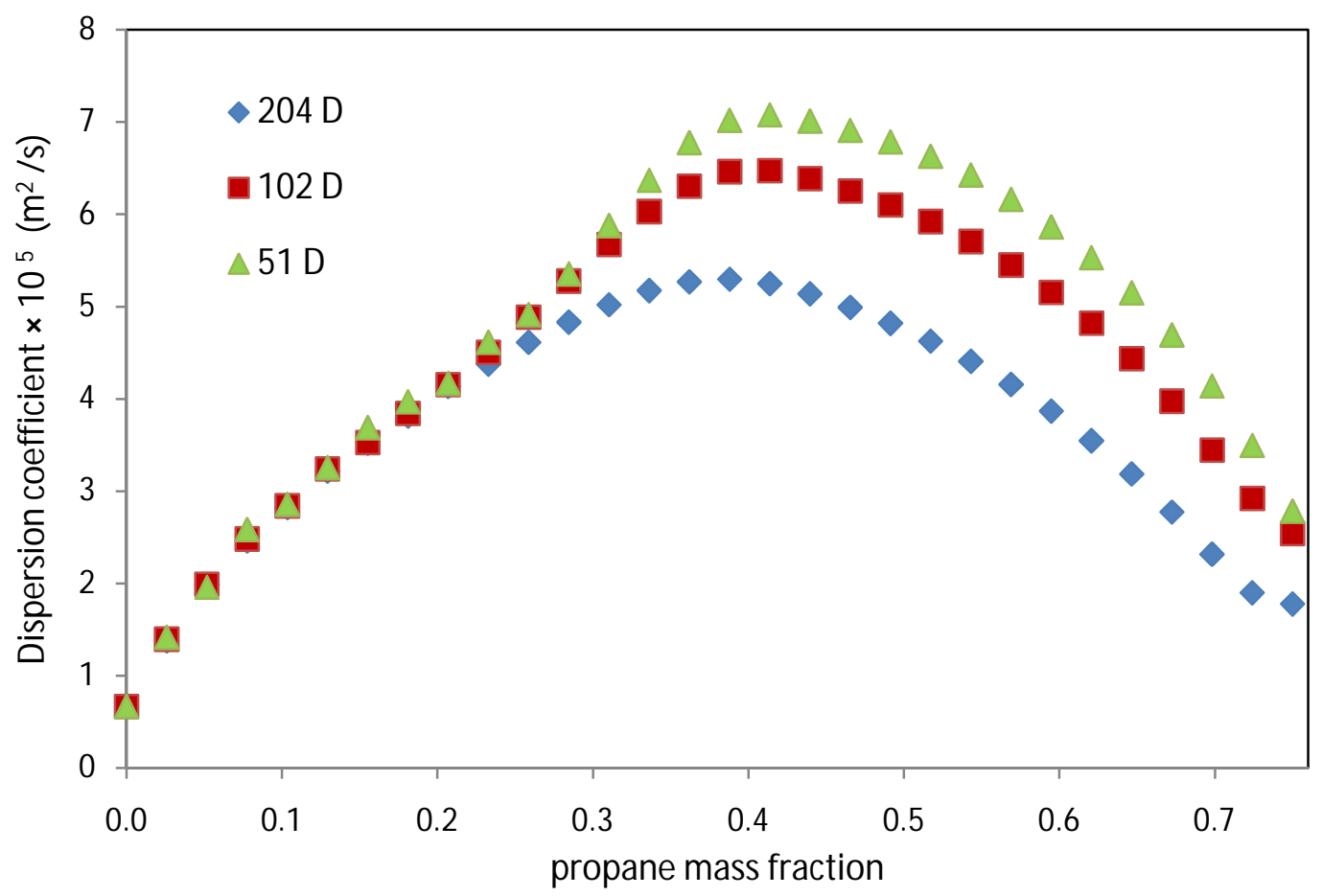

Figure 5.14 Dispersion coefficients of propane in heavy oil with three medium permeabilities (35 cm drainage height)

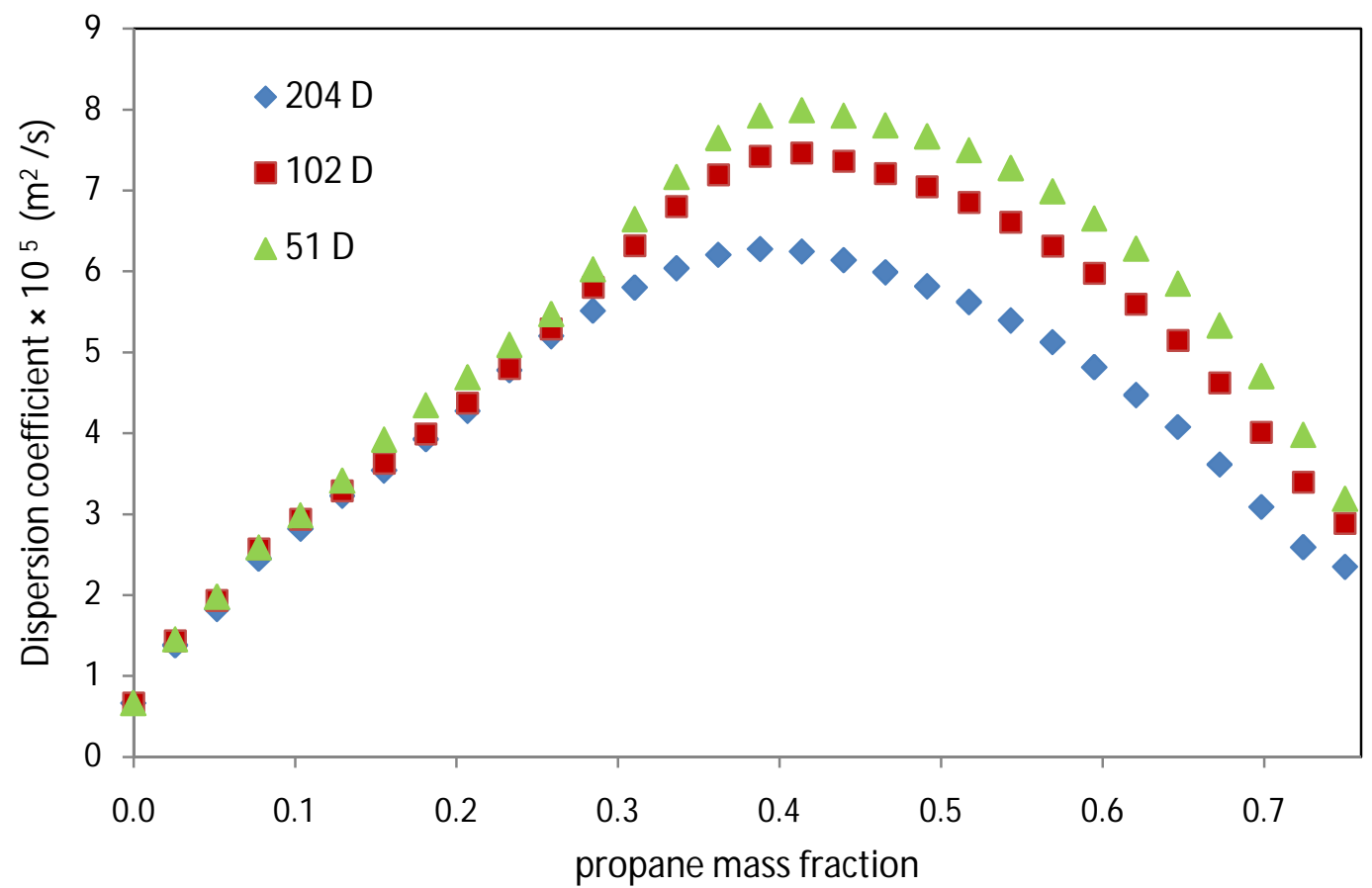

Figure 5.15 Dispersion coefficients of propane in heavy oil with three medium permeabilities (45 cm drainage height) 
As shown in Figures 5.16-5.18, the predicted cumulative live oil production is in a good agreement with the cumulative experimental production rate during the operation time of 60 minutes for the three packs permeabilities with drainage heights of 25,35 , and $45 \mathrm{~cm}$ correspondingly.

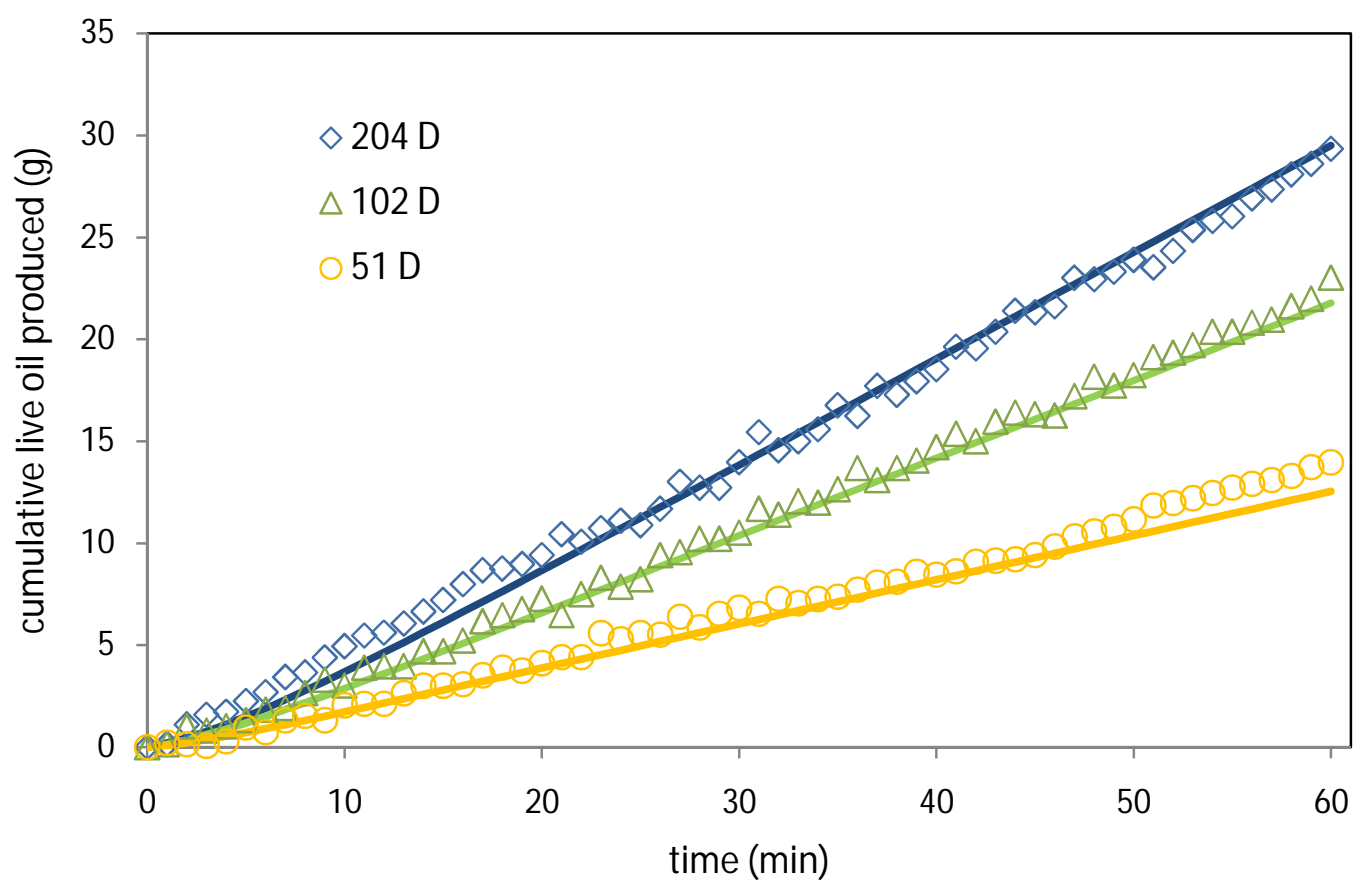

Figure 5.16 Experimental and calculated cumulative mass of oil produced with time for three medium permeabilities ( $25 \mathrm{~cm}$ drainage height) 


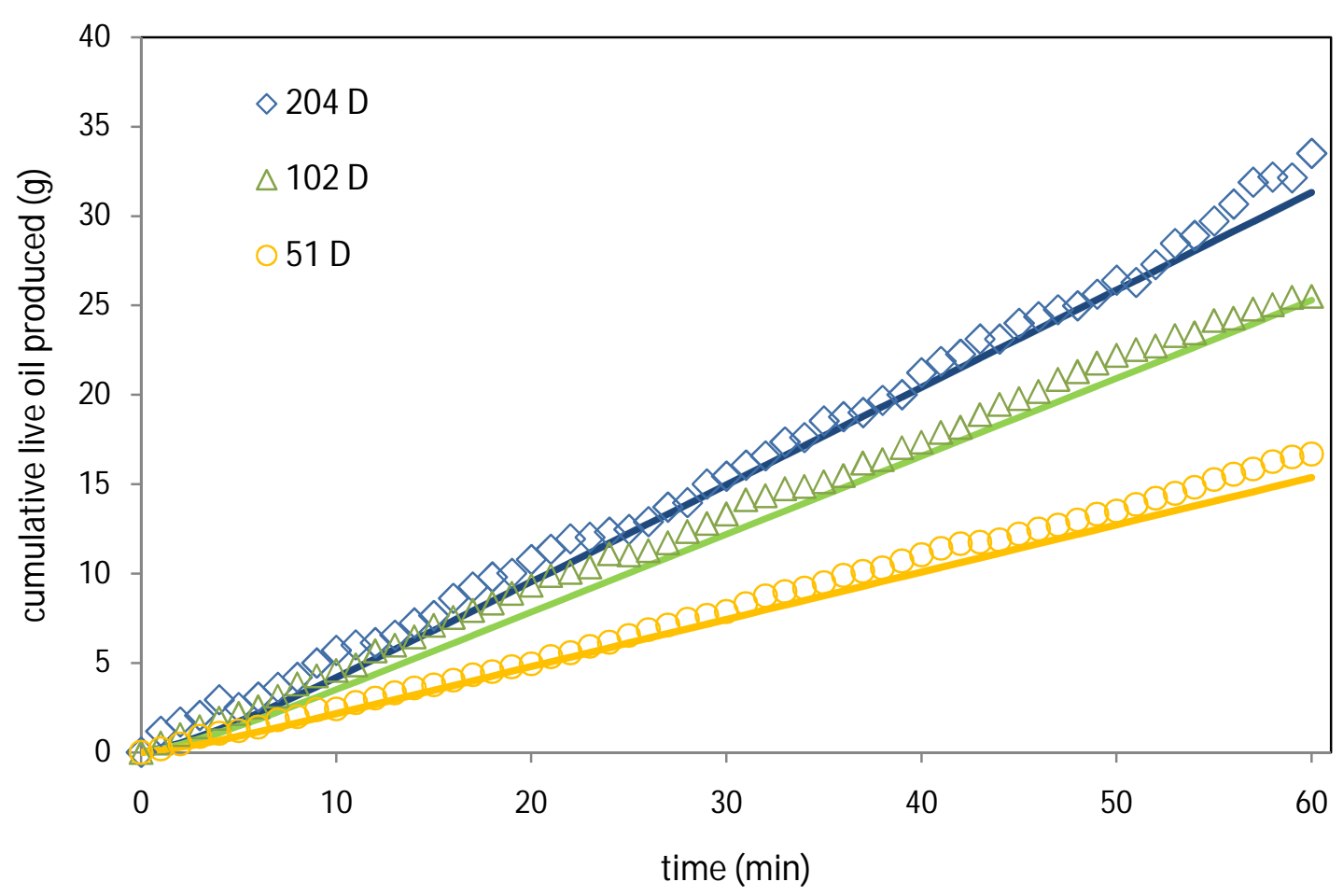

Figure 5.17 Experimental and calculated cumulative mass of oil produced with time for three medium permeabilities (35 $\mathrm{cm}$ drainage height)

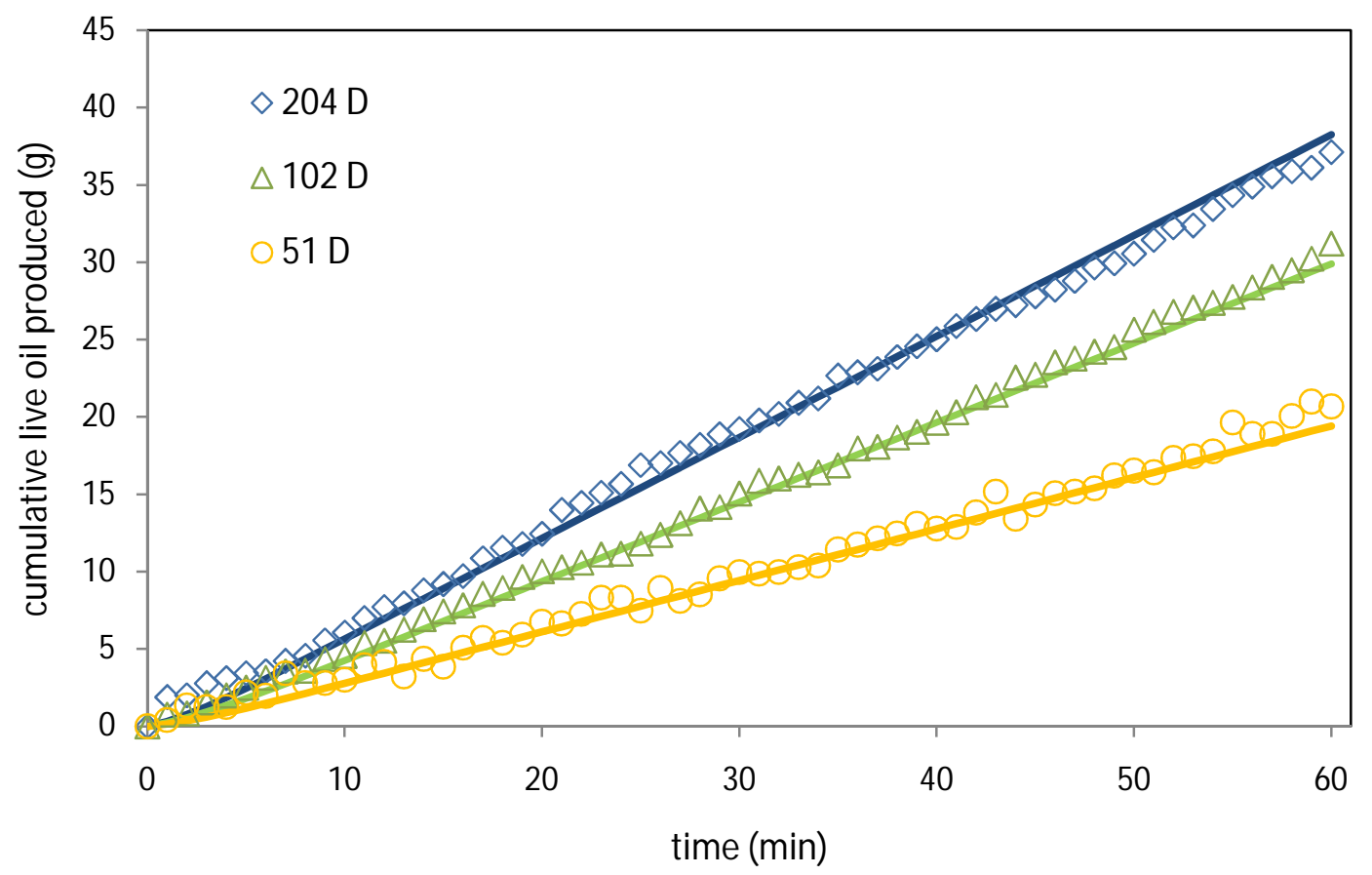

Figure 5.18 Experimental and calculated cumulative mass of oil produced with time for three medium permeabilities ( $45 \mathrm{~cm}$ drainage height) 
The average and maximum values of dispersion coefficient of propane in heavy oil and the corresponding propane mass fraction for the various physical models are shown in Table 5.9. In the range of 204-51 D and $25 \mathrm{~cm}$ drainage height, the dispersion values of propane lie between $0.5 \times 10^{-5}-6.09 \times 10^{-5} \mathrm{~m}^{2} / \mathrm{s}$. Its peak dispersion occurs in the gas mass fraction range of 0.336-0.413. In the range of 204-51 D and $35 \mathrm{~cm}$ drainage height, the dispersion values of propane lie between $0.67 \times 10^{-5}-7.083 \times 10^{-5} \mathrm{~m}^{2} / \mathrm{s}$. Its peak dispersion occurs in the gas mass fraction range of $0.387-0.414$. In the range of 204-51 D and $45 \mathrm{~cm}$ drainage height, the dispersion values of propane lie between $0.67 \times 10^{-5}-7.993 \times 10^{-5} \mathrm{~m}^{2} / \mathrm{s}$. Its peak dispersion occurs in the gas mass fraction range of $0.413-0.414$.

Table 5.9 Maximum and average dispersion values with corresponding mass fraction for different drainage heights

\begin{tabular}{|c|c|c|c|c|}
\hline $\begin{array}{l}\text { Drainage height } \\
\qquad(\mathrm{cm})\end{array}$ & $\begin{array}{l}\text { Packed medium permeability } \\
\text { (Darcy) }\end{array}$ & $\begin{array}{c}\text { Average } \\
\text { dispersion } \\
\left(\mathrm{m}^{2} / \mathrm{s}\right) \times 10^{5}\end{array}$ & $\begin{array}{l}\text { Maximum } \\
\text { dispersion } \\
\left(\mathrm{m}^{2} / \mathrm{s}\right) \times 10^{5}\end{array}$ & $\begin{array}{c}\text { Simulated } \\
\text { propane } \\
\text { mass } \\
\text { fraction }\end{array}$ \\
\hline \multirow{3}{*}{25} & 204 & 2.91 & 4.049 & 0.336 \\
\hline & 102 & 3.545 & 5.077 & 0.362 \\
\hline & 51 & 4.318 & 6.092 & 0.413 \\
\hline \multirow{3}{*}{35} & 204 & 3.722 & 5.31 & 0.387 \\
\hline & 102 & 4.428 & 6.466 & 0.413 \\
\hline & 51 & 4.795 & 7.083 & 0.414 \\
\hline \multirow{3}{*}{45} & 204 & 4.397 & 6.28 & 0.413 \\
\hline & 102 & 4.967 & 7.466 & 0.414 \\
\hline & 51 & 5.362 & 7.993 & 0.414 \\
\hline
\end{tabular}


It is observed that the average dispersion coefficients and the maximum dispersion coefficients increase with the decrease of the packed medium permeability. This behavior can be due to several factors: (i) As the size of glass beads decreases, the packing irregularity becomes more frequent, which, in turn, increases the specific surface area (interstitial surface area of the pore space per unit mass of porous medium). The increase in the specific surface area allows more solvent to contact heavy oil and diffuse into it, which in turn enhances mixing in the porous medium as reported by Perkins and Johnston (1963). (ii) The downward average drainage velocity of the live oil becomes lower with lower permeability as calculated in Section 5.1.3, thus, the interstitial velocity becomes lower in the pores of the porous medium, and the solvent has longer time to be in contact with the heavy oil producing more diffusion. (iii) The packing irregularity enhances velocity variations, which in turn, promotes convective mixing. Therefore, lower porous medium permeability produces lower live oil viscosity, and enhances dispersion. Thus, the impact of the change in permeability on the magnitude of convectional mixing is significant. Observations reported by El-Haj et al. (2009) confirmed dependence of dispersion on porous medium permeability.

Dispersion or mixing of a fluid in a porous medium is directly proportional to the particle size and fluid velocity (Brigham et al., 1961). Thus, decreasing the particle size will reduce the pores throat size in the porous medium, which in turn lowers the fluid velocity as well as the dispersion. In contrast, in this work, decreasing the particle size of the glass beads enhances dispersion. This is mainly due to the decrease in the fluid viscosity, which enhances more solvent's molecules to disperse in the heavy oil. Thus, dispersion is promoted with lower permeability.

\subsection{Drainage Height Effect on Concentration-Dependent Dispersion Coefficient}

The results in Section 5.1 have been used to determine the concentration-dependent dispersion coefficient of propane in heavy oil using a specific glass beads permeability to pack physical models of three drainage heights $(25,35$, and $45 \mathrm{~cm})$. Applying the developed algorithm as in Section 4.3.5, an improvement in $D(\omega)$ is accompanied by an iterative reduction of the objective functions as shown in Figure 5.19 for 204 D physical model. The objective functions values decrease monotonically to the minimum. 


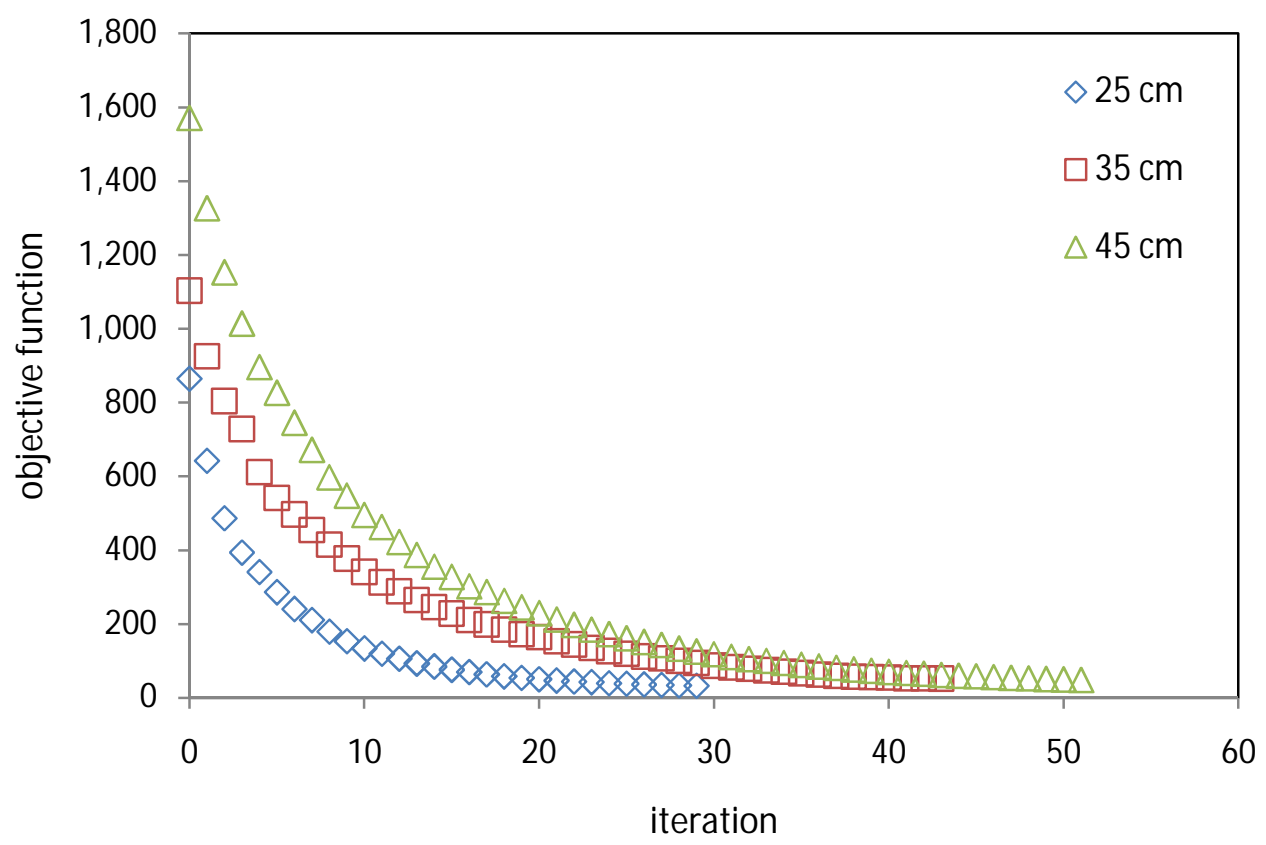

Figure 5.19 Objective functions versus iteration number for $204 \mathrm{D}$ model.

Figures 5.20-5.22 show the experimental stabilized live oil production rate compared to the model-predicted one corresponding to the optimal dispersion function for the three packs drainage heights with 204, 102, and 51 D permeability respectively. It is observed that the experimental and calculated live oil productions agree well. 


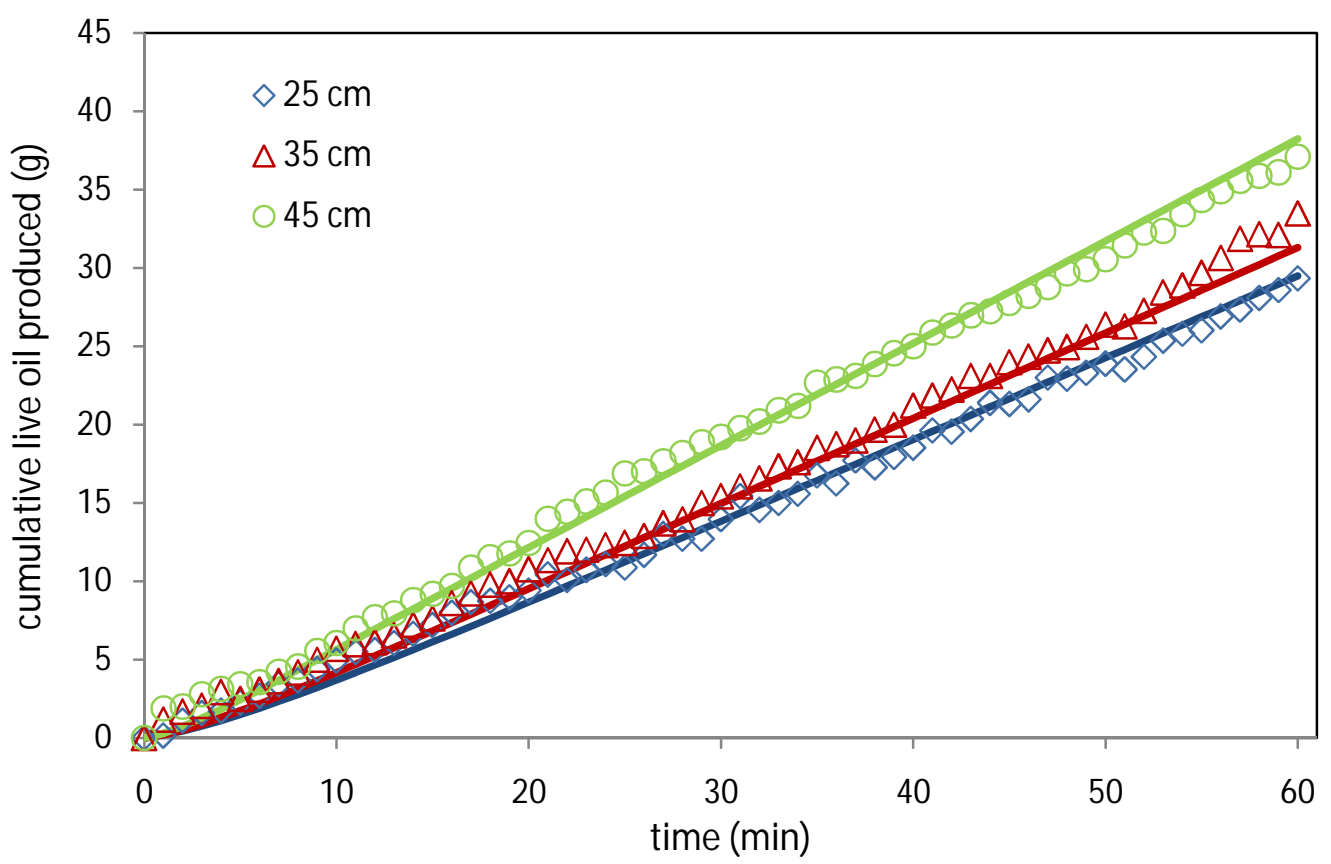

Figure 5.20 Experimental and calculated cumulative mass of oil produced with time for different model heights (204 D permeability)

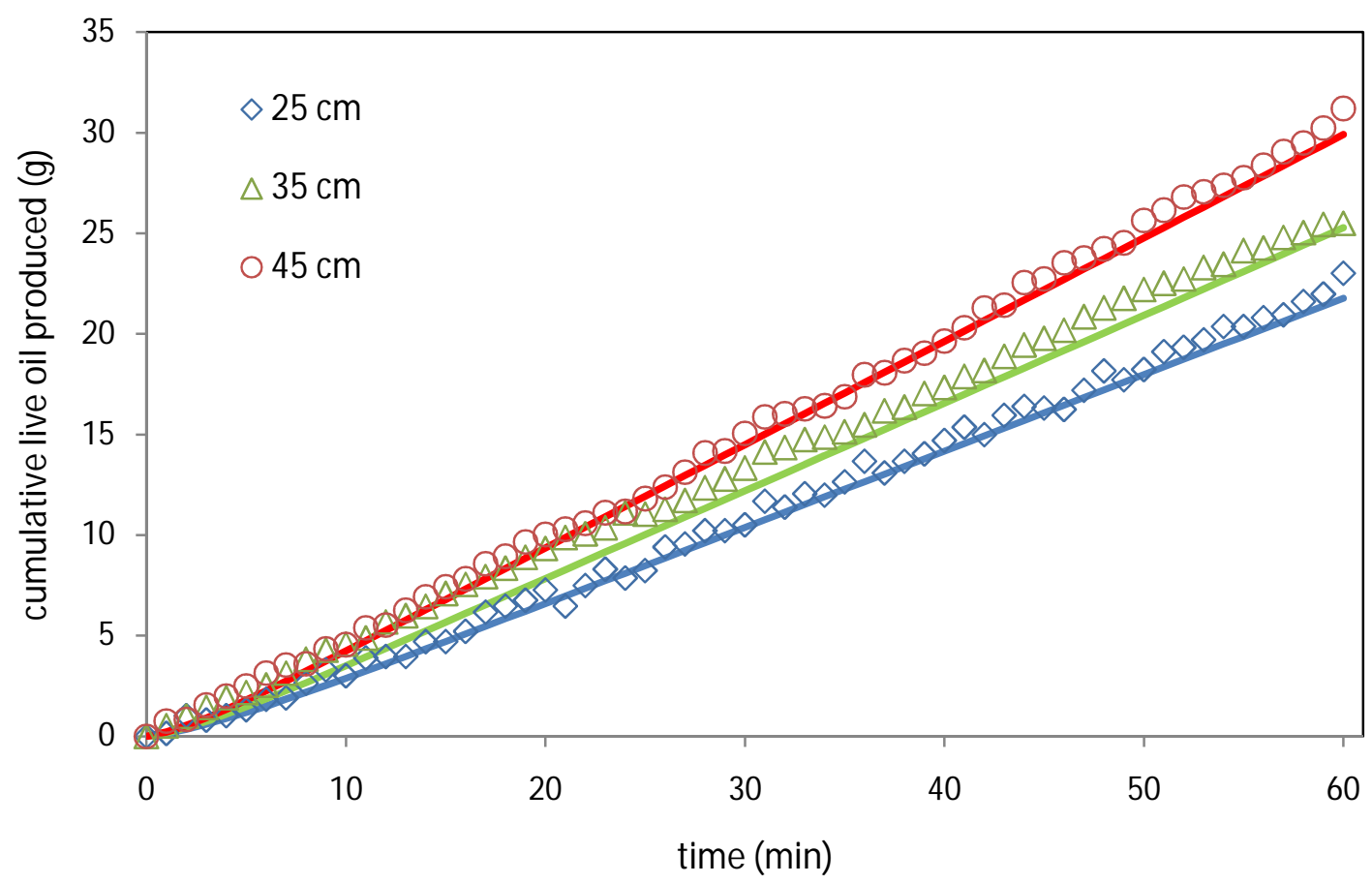

Figure 5.21 Experimental and calculated cumulative mass of oil produced with time for different model heights (102 D permeability) 


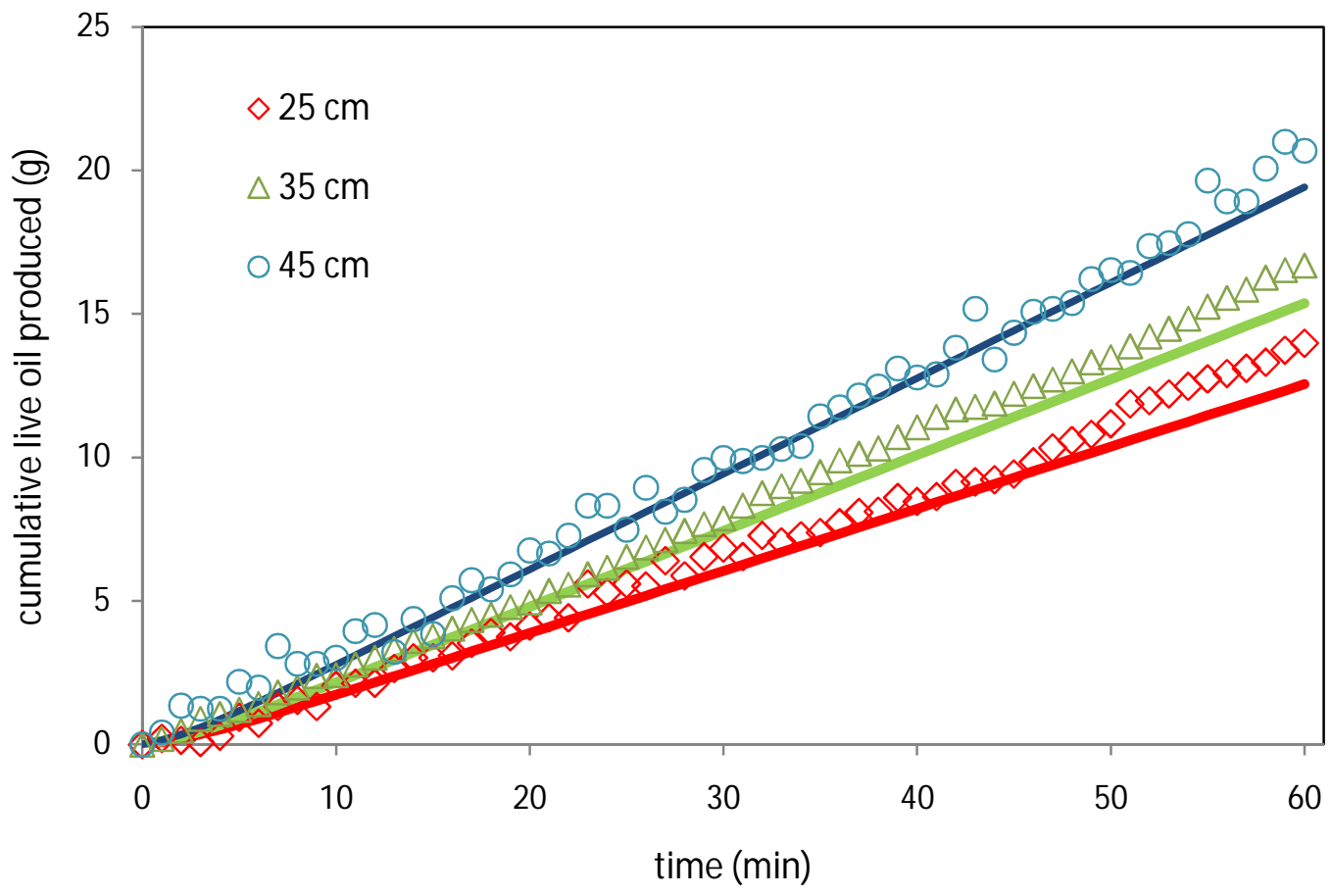

Figure 5.22 Experimental and calculated cumulative mass of oil produced with time for different model heights (51 D permeability)

Presented in Figure 5.23, those results for 204 D physical model show that with the propane mass fraction, the dispersion increases until it attains a maximum value before decreasing to lower values. The maximum dispersion coefficient is 8-13 times the minimum, thus indicating the strong concentration dependence of propane dispersion. Figures 5.24 and Figure 5.25 show the same behaviour of the dispersion functions for the various drainage heights with 102 and 51 D permeability. 


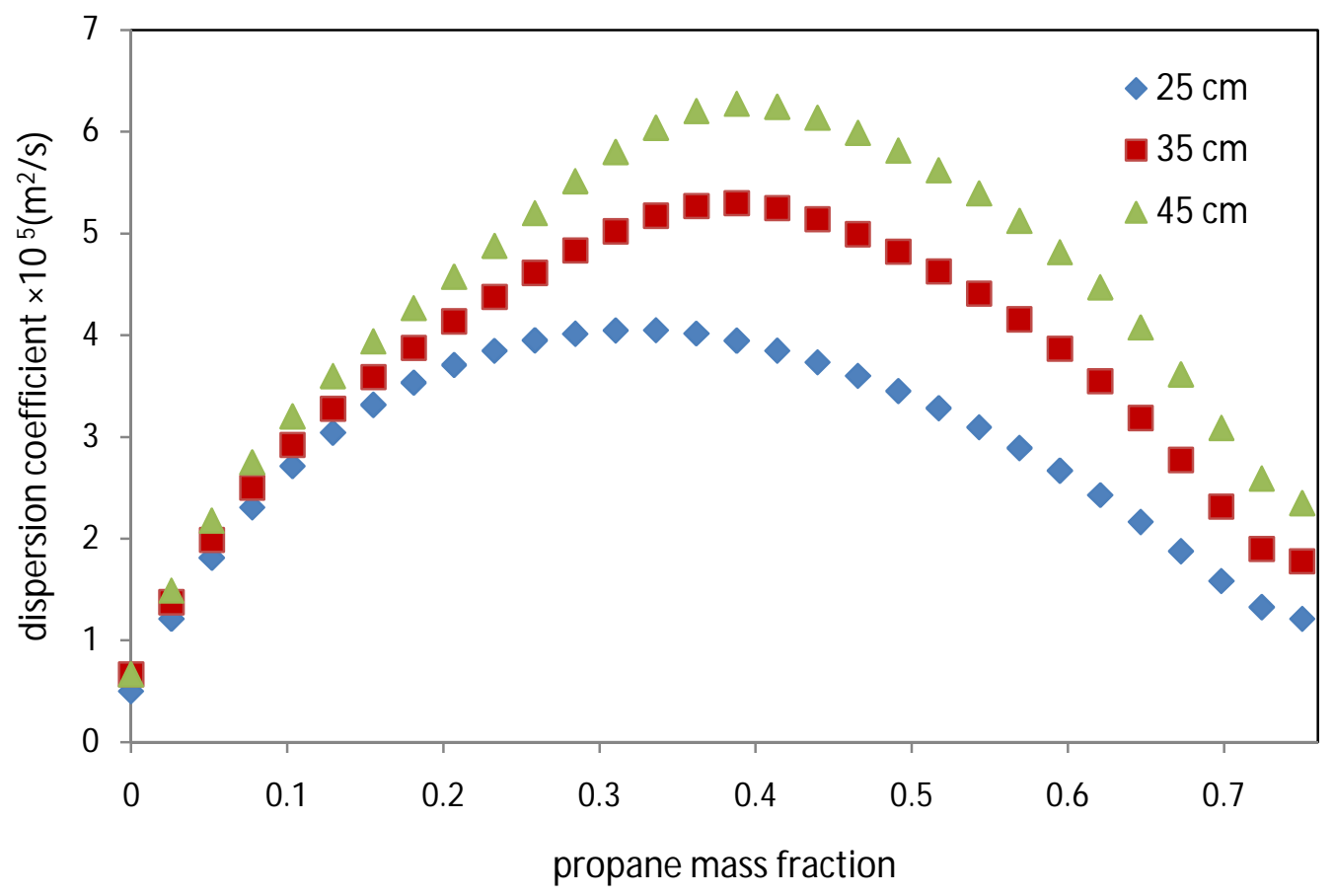

Figure 5.23 Dispersion coefficients of propane in heavy oil for $204 \mathrm{D}$ permeability with different model heights.

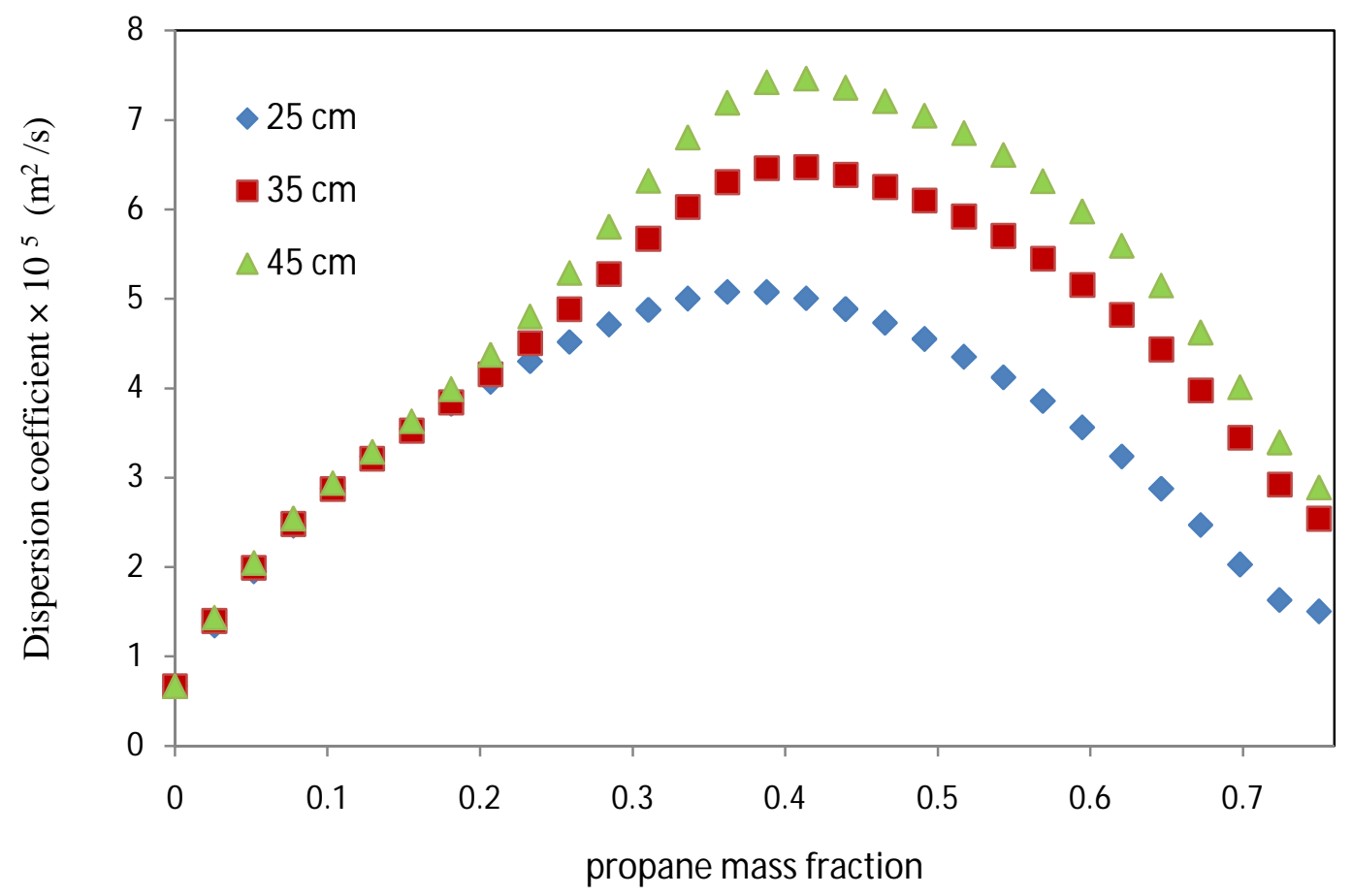

Figure 5.24 Dispersion coefficients of propane in heavy oil for $102 \mathrm{D}$ permeability with different model heights. 


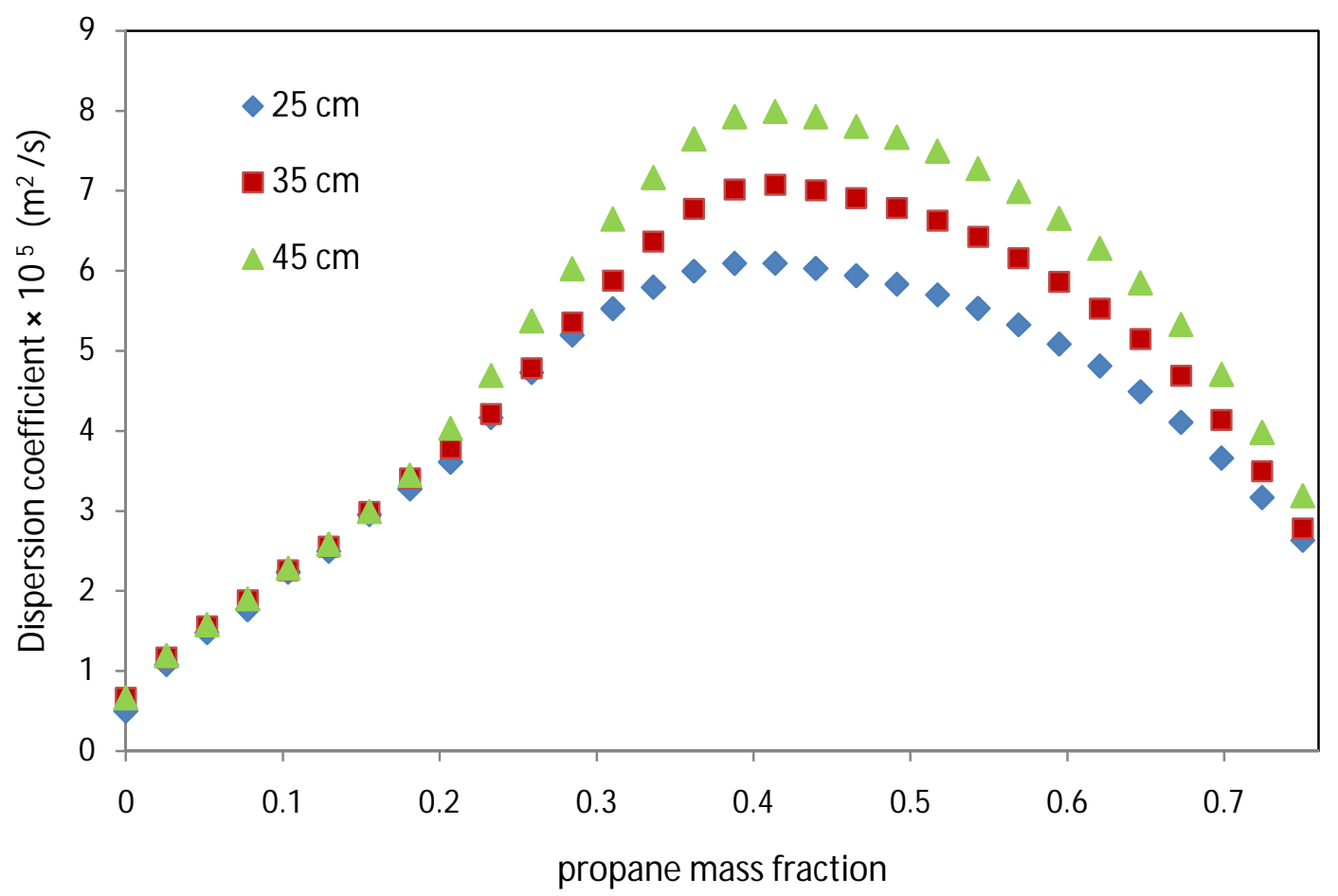

Figure 5.25 Dispersion coefficients of propane in heavy oil for $51 \mathrm{D}$ permeability with different model heights.

The average dispersion coefficient, maximum dispersion coefficient, and the corresponding propane mass fraction for the three different drainage heights are shown in Table 5.10. In the range of $25-45 \mathrm{~cm}$ and $204 \mathrm{D}$ permeability, the dispersion values of propane lie between $0.5 \times 10^{-5}$ $-6.27 \times 10^{-5} \mathrm{~m}^{2} / \mathrm{s}$. Its peak dispersion occurs in the gas mass fraction range of 0.336-0.414. In the range of $25-45 \mathrm{~cm}$ and $102 \mathrm{D}$ permeability, the dispersion values of propane lie between $0.67 \times 10^{-5}-7.46 \times 10^{-5} \mathrm{~m}^{2} / \mathrm{s}$. Its peak dispersion occurs in the gas mass fraction range of $0.362-$ 0.414 . In the range of $25-45 \mathrm{~cm}$ and $51 \mathrm{D}$ permeability, the dispersion values of propane lie between $0.5 \times 10^{-5}-7.993 \times 10^{-5} \mathrm{~m}^{2} / \mathrm{s}$. Its peak dispersion occurs in the gas mass fraction range of $0.413-0.414$. 
Table 5.10 Maximum and average dispersion and corresponding mass fraction for different packed medium permeabilities

\begin{tabular}{|c|c|c|c|c|}
\hline $\begin{array}{c}\text { Permeability } \\
\text { (Darcy) }\end{array}$ & $\begin{array}{c}\text { Drainage } \\
\text { height } \mathbf{( \mathbf { c m } )}\end{array}$ & $\begin{array}{c}\text { Average dispersion } \\
\left(\mathbf{m}^{2} / \mathbf{s}\right) \times 1 \mathbf{1}^{\mathbf{5}}\end{array}$ & $\begin{array}{c}\text { Maximum dispersion } \\
\left(\mathbf{m}^{2} / \mathbf{s}\right) \times 10^{\mathbf{5}}\end{array}$ & $\begin{array}{c}\text { Simulated } \\
\text { propane } \\
\text { mass } \\
\text { fraction }\end{array}$ \\
\hline \multirow{2}{*}{$\mathbf{2 0 4}$} & $\mathbf{2 5}$ & 2.91 & 4.049 & 0.336 \\
& $\mathbf{3 5}$ & 3.722 & 5.31 & 0.388 \\
& $\mathbf{4 5}$ & 4.397 & 6.28 & 0.414 \\
\hline \multirow{2}{*}{$\mathbf{1 0 2}$} & $\mathbf{2 5}$ & 3.545 & 5.077 & 0.362 \\
& $\mathbf{3 5}$ & 4.428 & 6.466 & 0.4137 \\
& $\mathbf{4 5}$ & 4.967 & 7.466 & 0.414 \\
\hline \multirow{2}{*}{$\mathbf{5 1}$} & $\mathbf{2 5}$ & 4.318 & 6.092 & 0.413 \\
& $\mathbf{3 5}$ & 4.795 & 7.083 & 0.414 \\
& $\mathbf{4 5}$ & 5.362 & 7.993 & 0.414 \\
\hline
\end{tabular}

It is observed that the average and maximum dispersion coefficient values increase with the drainage height of the packed medium. This behaviour can be explained as longer drainage height increases the diluted oil path length, exposure time to solvent, and contact surface area (solvent-live oil interface during the diluted oil flow along the length of the draining edge). The aforementioned effects will enhance the transfer of solvent in the heavy oil. As more solvent is dissolved in live oil, more reduction in live oil viscosity is expected. Thus, an increase in the average drainage velocity was produced as calculated in Table 5.5. Figure 5.26 shows the reduction in the average measured live oil viscosity with drainage height, which causes higher average drainage velocity of the live oil. 


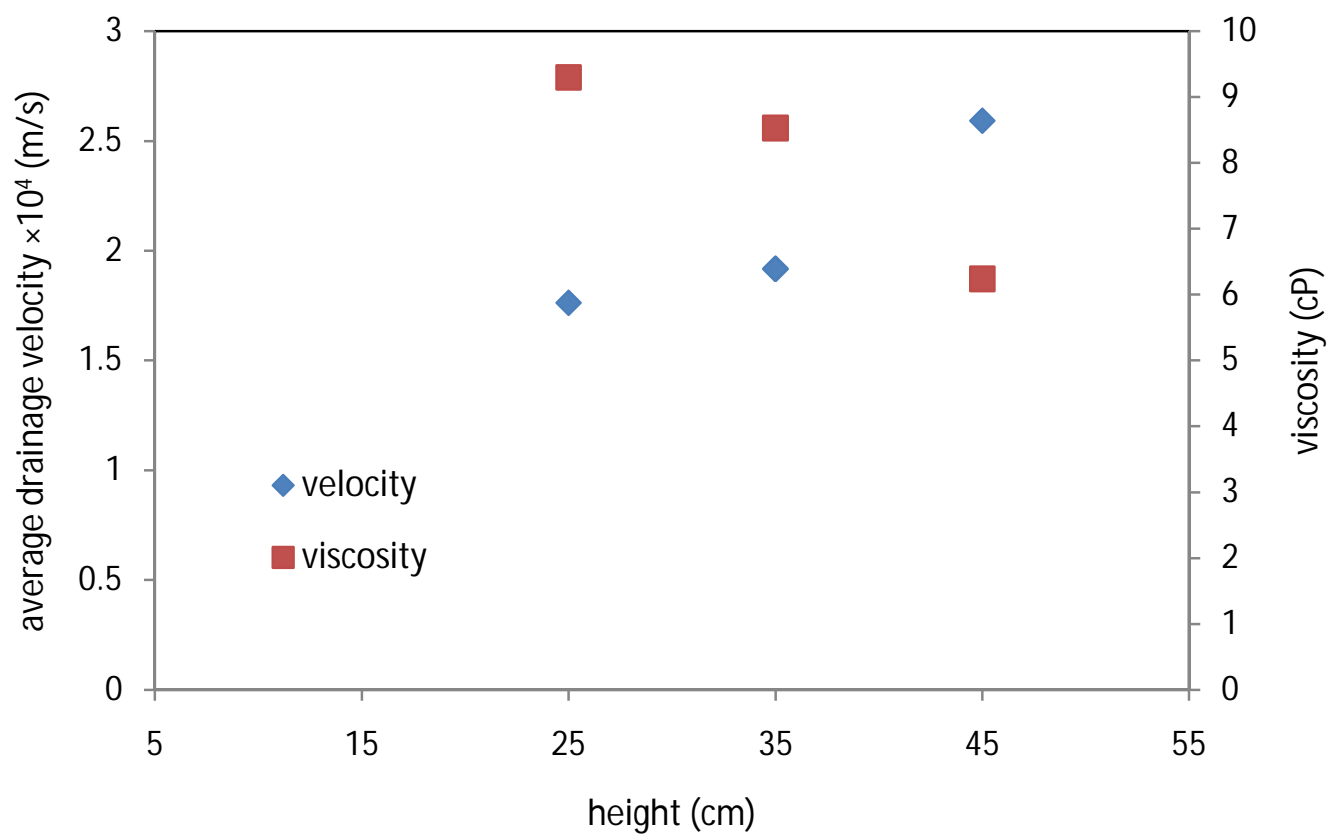

Figure 5.26 Average live oil viscosity and drainage velocity versus drainage height (204 D)

Higher average drainage velocity means higher interstitial velocity in the pores of the porous medium, which enhances velocity gradients, and heterogeneous flow paths causing more mechanical (convective) mixing that contributes to dispersion. Mechanical (convectional) dispersion causes the spreading of the solvent that implies redistribution of solvent concentrations that produces concentration gradients. Molecular diffusion tends to dampen down the concentration difference, which contributes also to dispersion.

Thus, higher drainage height augments mechanical dispersion (velocity dependent) and molecular diffusion. Therefore, the model height would be a significant parameter in the convective dispersion pertinent to the Vapex process, as the experimental results show. Experimental observations reported by other researchers confirmed that the model height increases the magnitude of convective dispersion in the Vapex process (Karmaker and Maini 2003; Yazdani and Maini 2005). 
Figure 5.27 shows how the average dispersion coefficient varies with the pack's permeability and drainage height. It is evident that the effect of porous medium permeability on average dispersion values as well as the drainage height is significant.

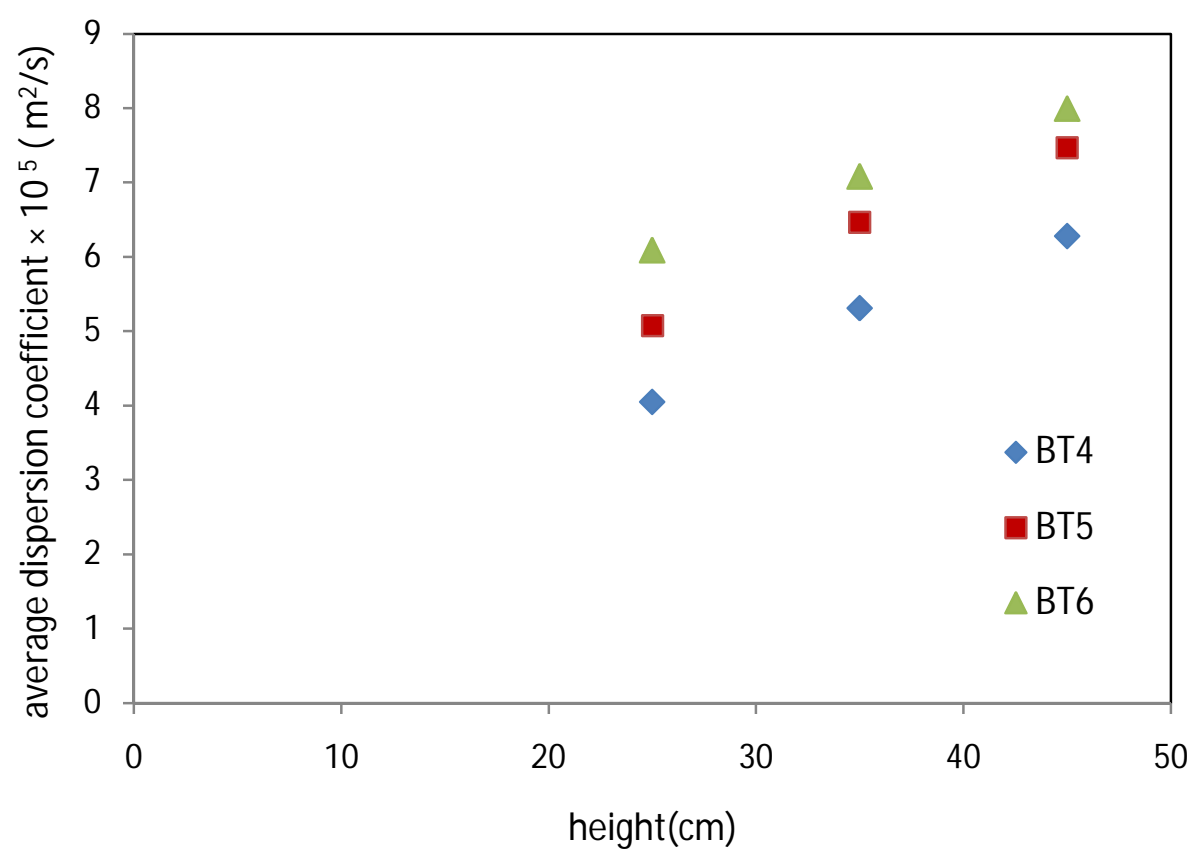

Figure 5.27 Average dispersion coefficients versus drainage height with various permeabilities.

Figure 5.28 below shows the average dispersion coefficients versus the average propane mass fraction obtained experimentally. For all experiments, propane average dispersion values determined ranges between $2.91 \times 10^{-5}-5.36 \times 10^{-5} \mathrm{~m}^{2} / \mathrm{s}$ and producing live oil with propane mass fraction that varied in the range of $0.285-0.441$. 


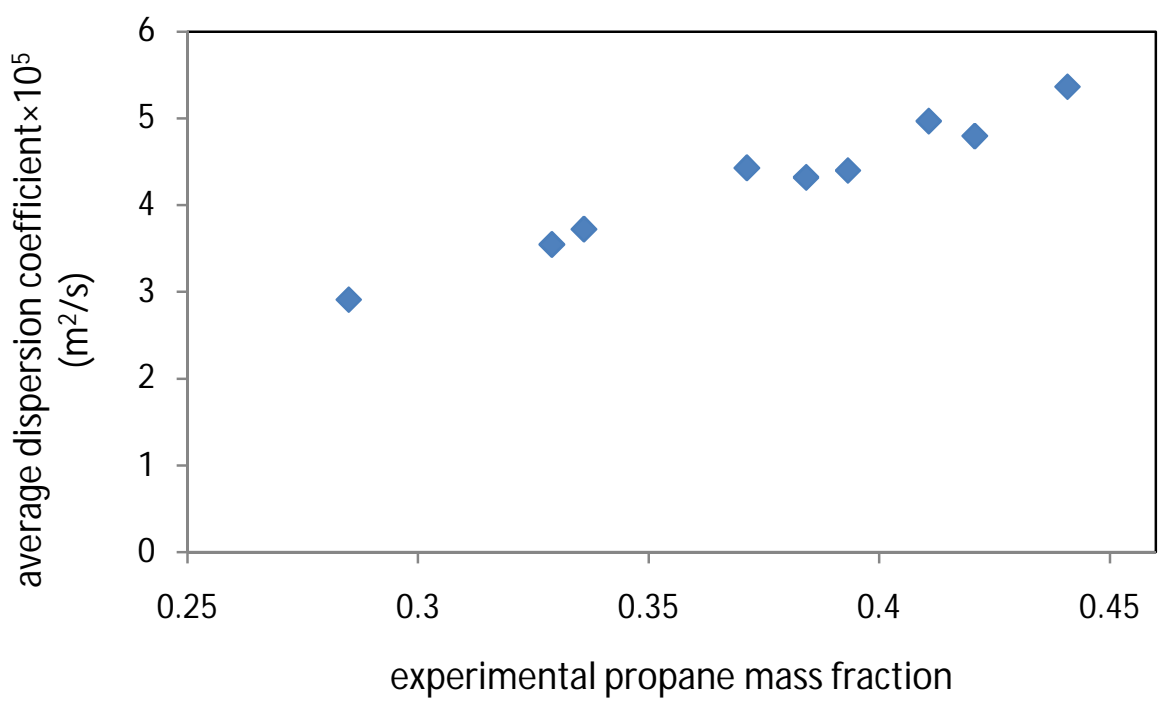

Figure 5.28 Average dispersion versus experimental average propane mass fraction

Figure 5.29 shows a portion of the dispersion function for all experiments. This portion represents the range of simulated propane mass fraction that corresponds to the maximum dispersion value. The dashed circle represents the maximum dispersion value for each experiment. Thus, for the same heavy oil and operating conditions, to maximize solvent uptake by the heavy oil and oil production as a consequence, solvent injection rates should be such that average solvent mass fraction in the heavy oil is in the range of the optimal solvent mass fraction (0.310-0.465) corresponding to the peak value of dispersion. 


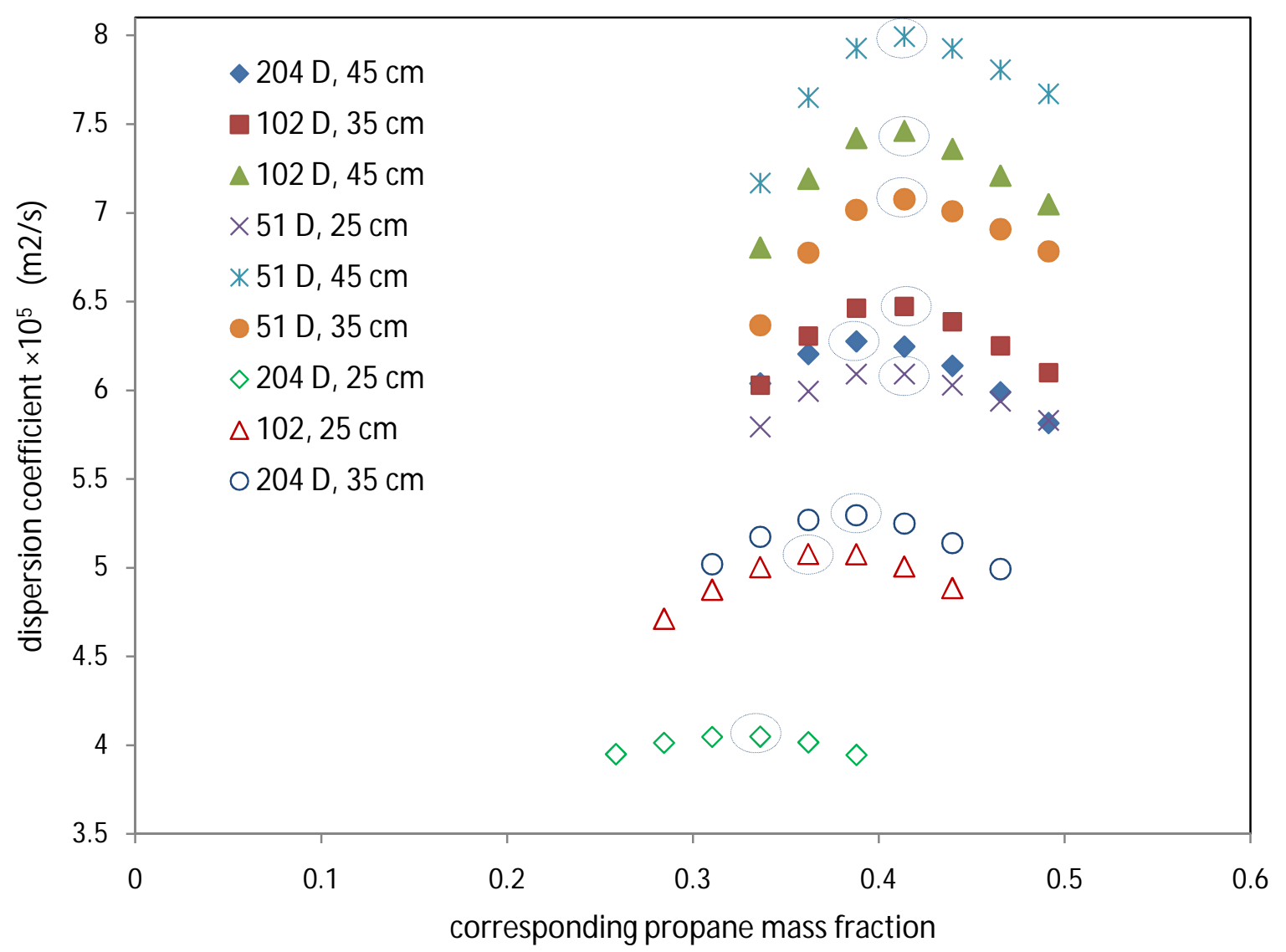

Figure 5.29 Propane dispersion versus corresponding propane mass fraction

\subsection{Permeability and Drainage Height Effect on Calculated Bitumen Height}

Applying the optimal values of $\omega_{\text {int }}$ and $D(\omega)$ for each experiment, the change in bitumen height with radial distance calculated at different times for the symmetrical vertical half of the physical model is shown in Figures 5.30 and 5.31. Figure 5.30 compares simulated bitumen heights in the porous medium of 204, 102 and 51 Darcy permeability with $25 \mathrm{~cm}$ drainage height. The plots in Figure 5.30 show that the simulated bitumen heights in the porous medium move in a relatively stable manner result a constant Vapex interface shape. Moreover, the bitumen height decreases faster as live oil production increases with the medium permeability. 

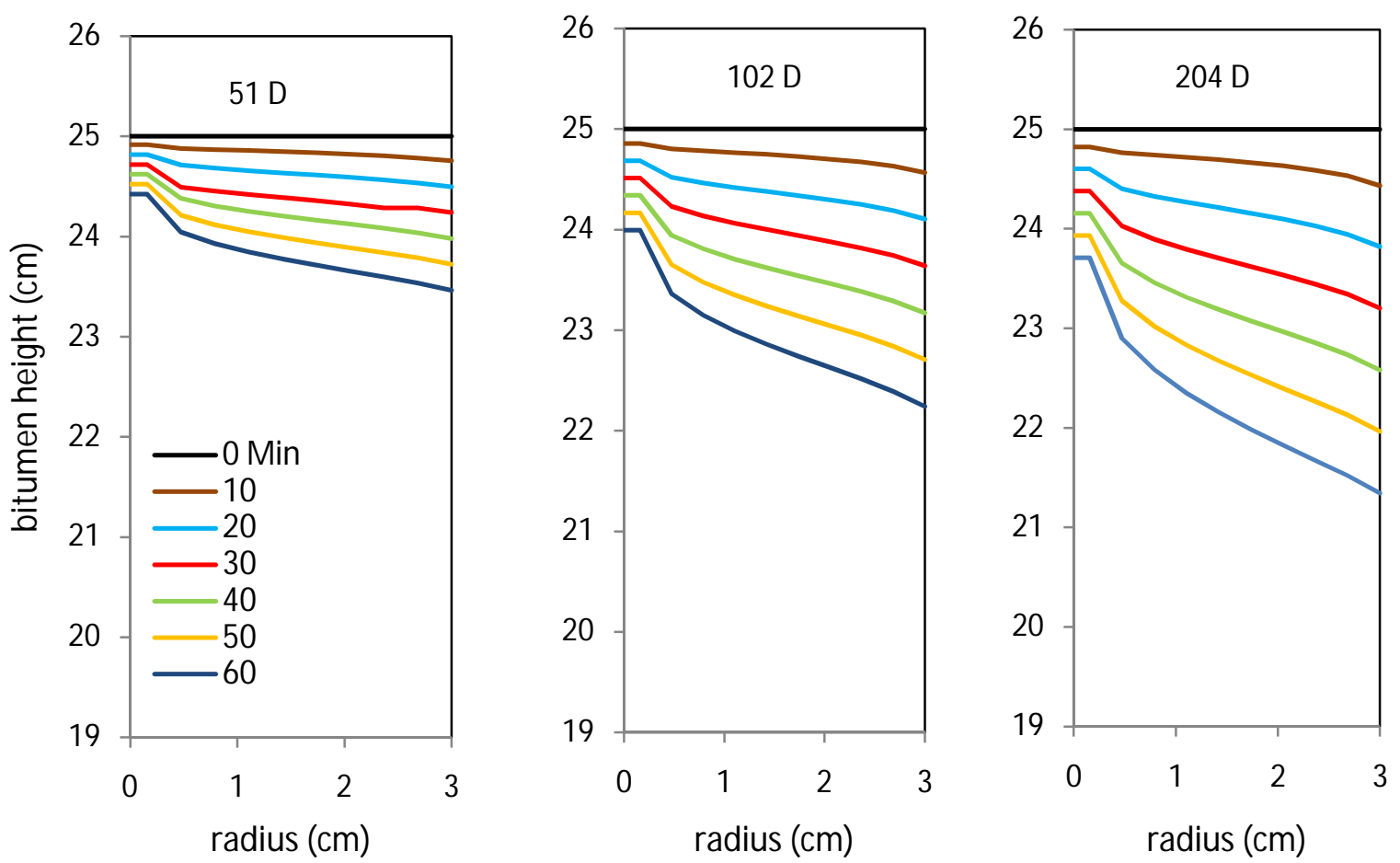

Figure 5.30 The change in bitumen height with time (204,102 and $51 \mathrm{D})$ and drainage height of $25 \mathrm{~cm}$.

Figure 5.31 compares simulated bitumen heights in the porous medium of 25,35 , and $45 \mathrm{~cm}$ drainage heights with $102 \mathrm{D}$ permeability. The plots in Figure 5.31 show that the bitumen height decreases faster as live oil production increases with the packing drainage height. 


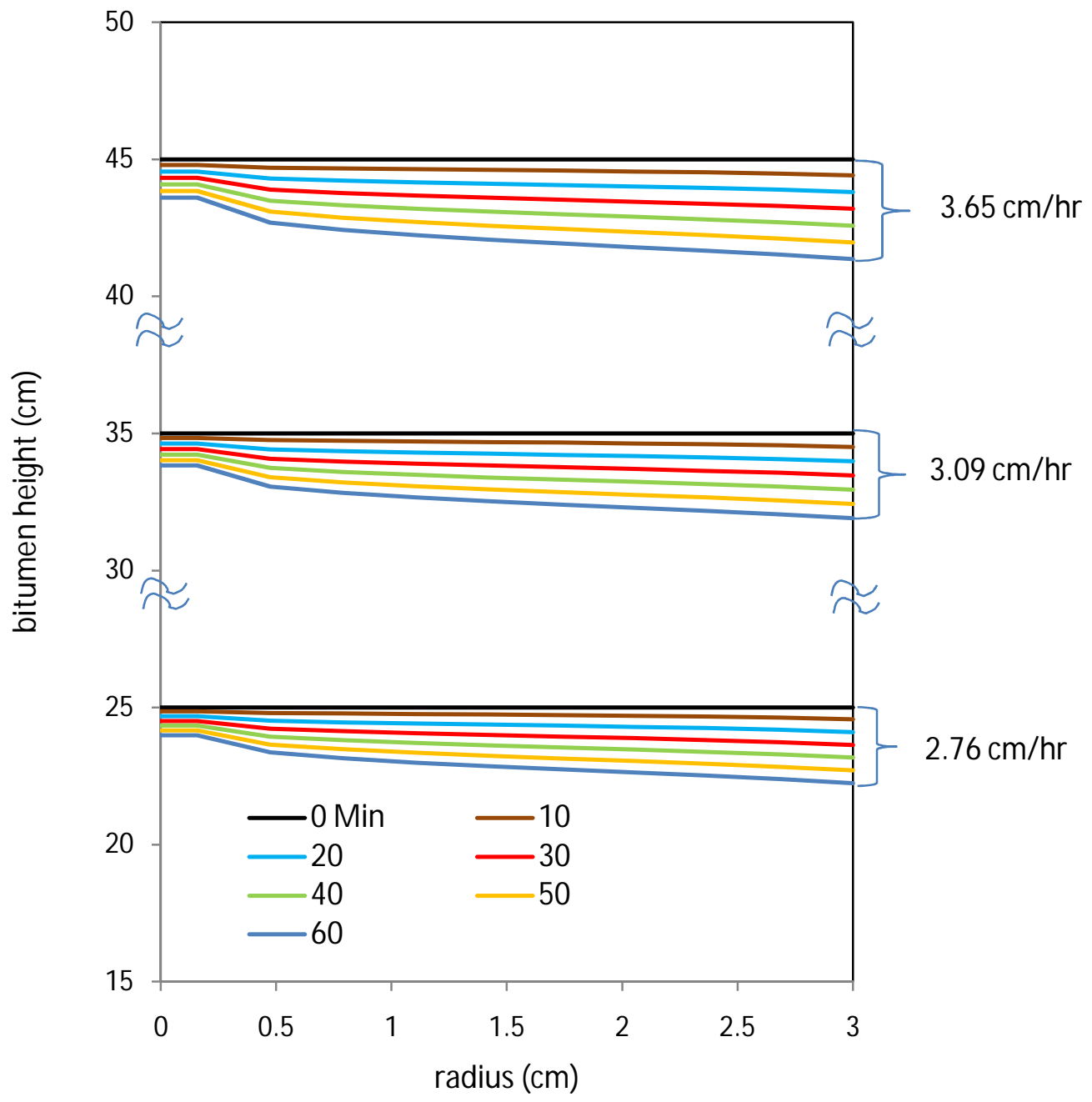

Figure 5.31 The change in bitumen height with time $(25,35$ and $45 \mathrm{~cm})$ and packing permeability of $102 \mathrm{D}$.

\subsection{Correlation of Dispersion Data}

The dispersion data is now presented in a form that can be easily incorporated into engineering calculations. The dispersion data are correlated as a function of propane mass fraction in heavy oil and drainage height; or porous medium permeability.

TableCurve $3 \mathrm{D}^{\circledR}$ was utilized to fit dispersion data as a function of propane mass fraction in heavy oil with permeability or drainage height of the packed medium using as few parameters as 
possible and an acceptable goodness of fit. Several types of functions and their combinations were tested. The following function captures propane dispersion as a function of mass fraction and permeability at a given packed medium drainage height:

$D=a+b \omega+c K+d \omega^{2}+e K^{2}+f \omega K$

where $K$ is the medium permeability, and $\omega$ is propane mass fraction in heavy oil. Table 5.11 lists the regressed values of the parameters $a, b, c, d, e$ and $f$.

Table 5.11 Regressed values of the parameters in Equation (5.2)

\begin{tabular}{|c|c|c|c|}
\hline \begin{tabular}{c} 
Drainage height $(\mathrm{cm})$ \\
\multicolumn{2}{c|}{}
\end{tabular} & $\mathbf{2 5}$ & $\mathbf{3 5}$ & $\mathbf{4 5}$ \\
\hline Parameters & & & \\
\hline $\mathrm{a} \times 10^{6}$ & 16.3 & 3.64 & 2.59 \\
\hline $\mathrm{b} \times 10^{4}$ & 2.37 & 2.89 & 3.33 \\
\hline $\mathrm{c} \times 10^{8}$ & -15.9 & 0.64 & -2.10 \\
\hline $\mathrm{d} \times 10^{4}$ & -2.7 & -3.2 & -3.6 \\
\hline $\mathrm{e} \times 10^{10}$ & 5.79 & 0.170 & 1.41 \\
\hline $\mathrm{f} \times \mathbf{1 0}^{7}$ & -2.17 & -2.16 & -2.08 \\
\hline $\mathrm{r}^{2}$ & 0.9602 & 0.9719 & 0.9658 \\
\hline FSE $\times 10^{6}$ & 2.9215 & 2.8674 & 3.6613 \\
\hline$\omega$ range & $0-0.75$ & $0-0.75$ & $0-0.75$ \\
\hline Permeability, K (D) & $204-51$ & $204-51$ & $204-51$ \\
\hline
\end{tabular}

Similarly, the following equation correlated propane dispersion as a function of mass fraction and drainage height at a given packed medium permeability:

$D=a+b \omega+c H+d \omega^{2}+e H^{2}+f \omega H$ 
where $H$ is the drainage height, and $\omega$ is propane mass fraction in heavy oil. Table 5.12 lists the regressed values of the parameters $a, b, c, d, e$ and $f$.

Table 5.12 Regressed values of the parameters in Equations (5.3)

\begin{tabular}{|c|c|c|c|}
\hline \begin{tabular}{c} 
Permeability (D) \\
\hline Parameters
\end{tabular} & 204 & 102 & 51 \\
\hline a $\times 10^{6}$ & & & \\
\hline b $\times 10^{4}$ & -9.97 & -16.0 & 6.16 \\
\hline $\mathrm{c} \times 10^{7}$ & 1.75 & 2.04 & 2.66 \\
\hline $\mathrm{d} \times 10^{4}$ & 8.02 & 12.2 & -5.07 \\
\hline $\mathrm{e} \times 10^{9}$ & -2.8 & -3.3 & -3.5 \\
\hline $\mathrm{f} \times 10^{6}$ & -7.10 & -17.2 & 7.11 \\
\hline $\mathrm{r}^{2}$ & 1.18 & 1.84 & 1.23 \\
\hline FSE $\times 10^{6}$ & 0.9692 & 0.9637 & 0.9423 \\
\hline w range & 2.6038 & 3.3998 & 4.9586 \\
\hline drainage height, H (cm) & $0-0.75$ & $0-0.75$ & $0-0.75$ \\
\hline
\end{tabular}

It may be noted that Equations (5.2) and (5.3) represents the dispersion data within the specified range of propane mass fraction in heavy oil and drainage height or permeability. Any extrapolation should be done with caution. It should be used for interpolation purposes only.

\subsection{Significance of the Dispersion Data}

In this work, the dispersion values determined represent the "total" mixing results from all possible mechanisms enhancing mixing in the porous medium including diffusion, convective mixing, viscosity reduction, gravity action, surface renewal, capillary action, etc. The contribution of these factors cannot be isolated from the two major components of dispersion (diffusion and convective mixing). Thus, higher dispersion values mean higher mixing rates, which in turn imply higher rates of oil production. 
In this study, dependence of propane dispersion on concentration was considered since diffusion, viscosity and mobility (all embodied in dispersion) are strongly influenced by solvent concentration (Oballa and Butler 1989; Upreti and Mehrotra 2000, 2002). In addition, propaneheavy oil mixture is not dilute, thus, a constant diffusion coefficient value is not expected and can lead to erroneous results. The optimal control approach developed allows dispersion values to improve naturally and gradually.

The mathematical model was developed directly from the equation of continuity for propane in bitumen without using oversimplified assumptions. The continuity equation maintained dispersion and convection terms and was developed in terms of a concentration dependent dispersion.

Determination of the dispersion coefficient of propane in heavy oil was based on real experimental data. The model parameters utilized in the simulation were measured experimentally such as live oil production rates, live oil density and viscosity. A system-specific correlation representing the true behaviour of the viscosity reduction during Vapex was generated to assure the reliability of the mathematical model.

The determination of the dispersion values was treated as an optimal control problem where fundamental mathematical principles (calculus of variations) were applied to a mass transfer model that described the dynamics of the Vapex process to search for the optimal dispersion function. Thus, the optimally determined concentration-dependent dispersion coefficient for propane in heavy oil satisfied the agreement between numerically simulated production rates and the experimental ones. These tools should increase the degree of confidence in the determined dispersion coefficients since it was not such a simple history match determination.

This work, which determines propane dispersion not as a single value but a function of propane concentration in heavy oil, is the first step to capture one of the many complications involved in mass transfer in Vapex. The dispersion data would give new insights to quantify the contribution of other mechanisms that augment mixing in the porous medium such as solvent viscosity reduction and capillary action. 
The dispersion data provide insights into optimizing Vapex operations. For example, solvent should be injected at a rate that produces live oil in the reservoir with solvent mass fraction close to the optimal solvent mass fraction range corresponding to the maximum value of dispersion at the operating temperature and pressure. Maximum solvent uptake will yield higher oil production rates.

These developed correlations for propane dispersion coefficient, which encounter the effect of drainage height and porous medium permeability, can be readily used in the simulators at similar operating conditions to estimate dispersion coefficients of propane, which may generate more accurate simulations and implementations. 


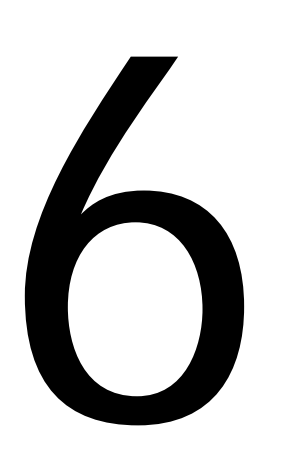

\section{Conclusions and Recommendations}

\subsection{Conclusions}

1. A novel technique was developed in this work to determine the dispersion coefficient of propane as a function of its concentration in heavy oil for Vapex experiments. The developed technique is a combined experimental-modelling-optimal control approach.

2. A two-dimensional convection-dispersion model was mathematically formulated in conjunction with Darcy's law to predict the live oil production rates. The model was developed from the equation of continuity for propane in bitumen and incorporates a concentration dependent dispersion. The reduction of bitumen height during Vapex, i.e. its moving boundary with time and space was taken into account.

3. An optimal control problem was formulated with objective functional subjected to the process constraints and the necessary conditions were derived utilizing the principles of calculus of variations. The difference in the predicted and experimentally measured oil production rates was minimized to determine not only the concentration-dependent dispersion coefficients of propane in heavy oil, but also the unknown propane interface concentration.

4. Nine experiments were conducted to measure the live oil production rates and live oil properties at $21^{\circ} \mathrm{C}$ and $0.790 \mathrm{MPa}$ pressure for various physical models. Each experiment was performed at least twice and the percentage error between the two experiments was found to be an average of $2.5 \%$. Live oil properties (propane mass fraction, live oil density and live oil viscosity) were measured. It was observed that live oil viscosity varied significantly with porous medium permeability and model drainage height. 
5. An optimal control algorithm involving Semi-Implicit Extrapolation method was used to integrate the model discretized non-dimensionalized differential equations to predict production rates. In addition, the BFGS method was utilized in the algorithm to improve the dispersion values to determine optimal propane dispersion coefficient as a function of its concentration in heavy oil.

6. The salient feature of this work is that the dispersion function is not restricted by a preconceived functional form but allowed to evolve naturally and gradually during the application of optimal control algorithm based on a reasonably rigorous mass transfer model.

7. The dispersion functions were observed to be unimodal functions of propane concentration in heavy oil. The dispersion coefficients of propane (at all concentrations) in heavy oil extracted from the numerical simulation of the Vapex experiments increased with lower porous medium permeability and higher drainage heights. In addition, it was found that the model height and the glass beads size (permeability) would have influences in convectional mixing in Vapex.

8. The optimal propane-bitumen interface mass fraction at the operating temperature and pressure was determined to be 0.76 . The dispersion of propane was found to be in the range of $0.5 \times 10^{-5}-7.99 \times 10^{-5} \mathrm{~m}^{2} / \mathrm{s}$.

9. The average values of the dispersion coefficient of propane determined were of two to three orders of magnitude higher than the reported ones by earlier investigators, and underscore the strong effect of mechanisms induced mixing in Vapex.

10. Propane dispersion was correlated as a function of (i) propane mass fraction in heavy oil and drainage height, and (ii) propane mass fraction in heavy oil and porous medium permeability.

11. The experiments conducted with different glass beads packs permeability and drainage heights confirmed the dependence of the oil production rates on the permeability and drainage height of the packed physical model. 


\subsection{Recommendations}

1. The framework of optimal control, and the computational algorithm developed in this work could be applied to determine the concentration-dependent dispersion of other solvents used in the recovery of heavy oil and bitumen.

2. A similar series of experiments could be performed using higher drainage heights and lower permeabilities to see whether the same effect of model height and permeability is still maintained.

3. Further investigations can be carried out to determine how different viscosities of heavy oils affect the dispersion coefficient of propane and establish a function among these factors.

4. The dispersion function can be extended to include thermodynamic conditions by conducting similar experiments using higher temperatures and pressures. 


\section{References}

Abukhalifeh, H., Lohi, A., Upreti, S.R., 2009. A Novel Technique to Determine Concentration-Dependent Solvent Dispersion in Vapex. Energies, 2(4), 851-872.

Abukhalifeh, H., Lohi, A., Upreti, S.R., 2010. Permeability Effect on the ConcentrationDependent Dispersion Coefficient of Propane in Vapex. International Journal of Oil, Gas, and Coal Technology, in press.

Allen, J.C. and Redford, A.D., 1976. Combination Solvent-Noncondensable Gas Injection Method for Recovering Petroleum from Viscous Petroleum-Containing Formations Including Tar Sand Deposits, United States Patent No. 4109720, Texaco, New York, US application No. 740281 .

Allen, J.C., 1974. Gaseous Solvent Heavy Oil Recovery, Canadian Patent No. 446874, February 28.

Badamchi-Zadeh, A., Yarranton, H.W., Svrcek, W.Y., and Maini, B.B., 2009. Phase Behaviour and Physical Property Measurements for VAPEX Solvents: Part I. Propane and Athabasca Bitumen. Journal of Canadian Petroleum Technology, 48(1), 54-61.

Bader, G. and Deuflhard, P., 1983, Numerische Mathematik, vol.41, 373-398.

Bear, J. and Buchlin, J-M., 1991. Modelling and Applications of Transport Phenomena in Porous Media. Kluwer Academic Publisher, London, volume 5.

Bear, J., 1972. Dynamics of fluids in porous media. American Elsevier Publishing Company, New York, USA.

Blackwell, R.J., 1962. Laboratory Studies of Microscopic Dispersion Phenomena, Society of Petroleum Engineers Journal, March, 1-8.

Boustani, A. and Maini, B.B., 2001. The Role of Diffusion and Convective Dispersion in Vapor Extraction Process. Journal of Canadian Petroleum Technology, 40, 68-77.

Brigham, W.E., Reed P.W., Dew J.N., 1961. Experiments on mixing during miscible displacement in porous media. Society of Petroleum Engineers Journal, March, 1, 1-8.

Broyden, C.G., 1970. The Convergence of a Class of Double-rank Minimization Algorithms. $J$. Inst. Maths. Applics., 6, 76-90.

Butler, R.M. and Mokrys, I.J., 1989. Solvent Analog Model of Steam Assisted Gravity Drainage. AOSTRA Journal of research, 5, 17-32. 
Butler, R.M. and Mokrys, I.J. 1991. A New Process (VAPEX) for Recovering Heavy Oils using Hot Water and Hydrocarbon Vapor. Journal of Canadian Petroleum Technology, 30(1), 97-106.

Butler, R.M., McNab, G.S., Lo, N. Y., 1981. Theoretical Studies on the Gravity Drainage of Heavy Oil during Steam Heating. Canadian Journal of Chemical Engineering, 59, 455-60.

Courant, R. and Hilbert, D., 1953. Methods of Mathematical Physics, Interscience Publishers, New York, USA.

Cuthiell, D., McCarthy, C., Frauenfeld, T., Cameron, S. and Kissel, G., 2003. Investigation of the VAPEX Process Using CT Scanning and Numerical Simulation. Journal of Canadian Petroleum Technology, 42 (2), 41-49.

Das, S. K. and Butler, R. M., 1994. Effect of Asphaltene Deposition on the Vapex Process: A Preliminary Investigation using a Hele-Shaw Cell. Journal of Canadian Petroleum Technology. 33(6), 39-45.

Das, S.K. and Butler, R.M., 1996. Diffusion Coefficients of Propane and Butane in Peace River Bitumen. Canadian Journal of Chemical Engineering, 74, 985-992.

Das, S.K, and Butler, R.M., 1998. Mechanism of the Vapor Extraction Process for Heavy Oil and Bitumen. Journal of Petroleum Science and Engineering, 21, 43-59.

Das, S. K., 1995. In Situ Recovery of Heavy Oil and Bitumen Using Vaporized Hydrocarbon Solvents, Ph.D. thesis, Department of Chemical and Petroleum Engineering, University of Calgary, Calgary.

Das, S.K., 1998. VAPEX: An Efficient Process for the Recovery of Heavy Oil and Bitumen, SPE 50941, 232-237.

Das, S.K. , 2002. VAPEX- A Unique Canadian Technology. Journal of Canadian Petroleum Technology, 41(8), 32-34.

Das, S. K. 2005. Diffusion and Dispersion in the Simulation of VAPEX Process, SPE 97924, SPE International Thermal Operations and Heavy Oil Symposium, 1-3 November, Calgary, Canada, 1-6.

Denn, M.M., 1969. Optimization by Variational Methods, McGraw-Hill, New York.

Dullien F.A.L., 1992. Porous Media, Fluid Transport and Structure, 2nd Edition, Academic Press, New York, USA. 
Dunn, S.G., Nenniger, E.H., Rajan, V.S.V., 1989. A Study of Bitumen Recovery by Gravity Drainage using Low Temperature Soluble Gas Injection. Canadian Journal of Chemical Engineering, 67, 978-991.

EIA - Energy Information Administration, 2008. International Energy Outlook. Report \#:DOE/EIA-0484(2008), Department of Energy, United States Government, http://www.eia.doe.gov/oiaf/ieo (accessed 07April 2010).

El-Haj, R., Lohi, A., Upreti, S.R., 2009. Experimental Determination of Butane Dispersion in Vapor extraction of Heavy Oil and Bitumen. Journal of Petroleum Science and Engineering. 67, $41-47$.

Fisher, D.B., Singhal A.K., Das S.K., Goldman J., Jackson C., 2000. Use of Magnetic Resonance Imaging and Advanced Image Analysis as a Tool to Extract Mass Transfer Information from a 2D Physical Model of the Vapex Process. SPE 59330, 1-9.

Fletcher, R., 1970. A New Approach to Variable Metric Algorithms. Computer Journal, 13, 317-322.

Freitag, N.P., Sayegh S.G., Exelby R., 2005. A New Semiautomatic PVT Apparatus for Characterizing Vapex Systems. SPE 97783, 1-7.

Friedrich, K.J., 2005. Effects of a Non-Condensable Gas on the Vapex Process, Masters Thesis, University of Waterloo, Waterloo, ON, Canada.

Goldfarb, D., 1970. A Family of Variable Metric Updates Derived by Variational Means. Mathematics of Computing, 24, 23-26.

Greenkorn, R. A., 1983. Flow Phenomena in Porous Media, Mercel Dekker, Inc. New York.

Haghighat, P. and Maini, B. B., 2008. Role of Asphaltene Precipitation in Vapex Process. Proceedings: Canadian International Petroleum Conference, Calgary, AB, June 17-19, paper 2008-087, 1-9.

Hayduk, W., Castaneda, R., Bromfield, H. and Perras R.R., 1973. Diffusivities of Propane in Normal Paraffin, Chlorobenzene, and Butanol Solvents; AIChE Journal, 19(4) 859-861.

Hyduk, W. and Cheng S.C., 1971. Review of Relation between Diffusivity and Solvent Viscosity in Dilute Liquid Solutions. Chemical Engineering Science, 26, 635-646.

James, L.A., Chatzis, M.A., Ioannidis M.A., 2003. Determination of Diffusion Coefficient of Butane in Heavy Oil and the Mass Transfer Rate at the Pore Scale in Vapex. Petroleum Society's Canadian International Petroleum Conference, Paper 2003-204, Calgary, Alberta. June 10-12. 
Jiang, Q., and Butler, R.M., 1996. Selection of Well Configurations in Vapex Process. SPE 37145 .

Jin, W., 1999. Heavy Oil Recovery Using the VAPEX Process, Masters Thesis, University of Waterloo, Waterloo, ON, Canada.

John, A. K., 2008. Dispersion in Large Scale Permeable Media. PhD Thesis, The University of Texas at Austin, TX, U.S.A.

Kapadia, R., Upreti, S.R., Lohi, A., Chatzis I., 2006. Determination of Gas Dispersion in Vapor Extraction of Heavy Oil and Bitumen. Journal of Petroleum Science and Engineering, 51, 214-222.

Karmaker, K., Maini, B.B., 2003. Experimental Investigation of Oil Drainage Rates in the VAPEX Process for Heavy Oil and Bitumen Reservoirs, SPE 84198, 1-6.

Kirk, D.E., 1970. Optimal Control Theory, An Introduction. Dover Publications, New York, USA.

Lim, G.B., Kry, R.P., Harker, B.C., Jha, K.N., 1996. Three-Dimensional Scaled Physical Modelling of Solvent Vapor Extraction of Cold Lake Bitumen. Journal of Canadian Petroleum Technology, 35(4), 32-40.

Luo, H. and Kantzas, A., 2008. Investigation of Diffusion Coefficients of Heavy Oil and Hydrocarbon Solvent Systems in Porous Media. Proceedings: SPE Improved Oil Recovery Symposium, 19-23 April 2008, Tulsa, Oklahoma, USA, paper SPE 113995.

Luo, P. and Gu, Y., 2009. Characterization of a Heavy Oil-Propane System in the Presence or Absence of Asphaltene Precipitation. Fluid Phase Equilibria, 277 (1), 1-8.

Luo, P., Wang X., Gu Y., 2008. Asphaltene Precipitation and its Effects on the Vapor Extraction (VAPEX) Heavy Oil Recovery Process. Society of Petroleum Engineers Journal, PS008-314.

Luo, P., Yang C. and Gu, Y., 2007. Enhanced Solvent Dissolution into In-Situ Upgraded Heavy Oil Under Different Pressures. Fluid Phase Equilibria, 252, 143-151.

Moghadam, S., Nobakht, M., Gu, Y., 2009. Theoretical and Physical Modeling of a Solvent Vapor Extraction (VAPEX) Process for Heavy Oil Recovery. Journal of Petroleum Science and Engineering, 65, 93-104.

Mokrys, I.J. and Butler, R.M., 1993. In-Situ Upgrading of Heavy Oils and Bitumen by Propane Deasphalting: The Vapex Process. SPE 25452, March 21-23. 
Nenniger, E.H., 1979. Canadian Patent CA1059432, Hydrocarbon Recovery, Hatch \& Associates, 31 July.

Nghiem L. X., Kohse B.F., Sammon, P.H. 2001. Compositional Simulation of the VAPEX Process. Journal of Canadian Petroleum Technology. 40(8), 54-61.

Nghiem L. X., Sammon, P.H., Kohse B.F., 2001. Modeling Asphaltene Precipitation and Dispersive Mixing in the Vapex Process. SPE Reservoir Simulation Simposium Proceedings.

Oballa, V. and Butler, R. M., 1989. An Experimental Study of Diffusion in the BitumenToluene System. Journal of Canadian Petroleum Technology, 28, 63-69.

Oduntan, A.R. 2001. Heavy Oil Recovery Using the VAPEX Process: Scale Up and Mass Transfer Issues. Master's Thesis, University of Waterloo, Waterloo, Canada.

Perkins, T.K. and Johnston, O.C., 1963. A Review of Diffusion and Dispersion in Porous Media. SPE Journal, 3, 70-84.

Press, W.H., Teukolsky, S.A., Vetterling, W.T. , Flannery, B.P., 2002. Numerical Recipes in C++. The Art of Scientific Computing, Cambridge University Press: New York, USA, 2nd edition, 719-727.

Ramakrishnan, V., 2003. In situ Recovery of Heavy Oil by VAPEX using Propane. Master's Thesis, University of Waterloo, Waterloo, Canada.

Ramirez, F.W., 1987. Developments in Petroleum Science 21, Application of Optimal Control Theory to Enhanced Oil Recovery, Elsevier, New York.

Ray, W. H., 1981. Advanced Process Control, McGraw-Hill, New York.

Reid, R.C., Prausnitz, J.M., Poling, B.E., 1987. The properties of Gases and Liquids, Fourth Edition, McGraw-Hill, New York.

Schmidt, T., Leshchyshyn T. H., Puttagunta V.R., 1982. Diffusivity of Carbon Dioxide into Athabasca Bitumen. Petroleum Society of CIM., Paper No. 82-33-100.

Shanno, D.F., 1970. Conditioning of Quasi-Newton Methods for Function Minimization. Mathematics of Computing, 24, 647-656.

Smalley, C., 2000. Heavy Oil and Viscous Oil Chapter from Modern Petroleum Technology. R.A. Dawe, ed, John Wiley and Sons Ltd.

Speight, J. G., 2009. Enhanced Recovery Methods for Heavy Oil and Tar Sands. Gulf Publishing Company,USA. 
Tharanivasan, A.K., Yang C., Gu Y., 2006. Measurements of Molecular Diffusion Coefficients of Carbon Dioxide, Methane, and Prpane in Heavy Oil under Reservoir Conditions. Energy \& Fuels, 20, 2509-2517.

Taylor, G., 1953. Dispersion of Soluble Matter in Solvent Flowing Slowly Through a Tube. Proceedings of the Royal Society of London. Series A: Mathematical and Physical Sciences, 219, 186-203.

Upreti, S.R. and Mehrotra, A.K., 2000. Experimental Measurement of Gas Diffusivity in Bitumen: Results for Carbon Dioxide. Ind. Eng. Chem. Res., 39, 1080-1087.

Upreti, S.R. and Mehrotra, A.K., 2002. Diffusivity of $\mathrm{CO}_{2}, \mathrm{CH}_{4}, \mathrm{C}_{2} \mathrm{H}_{6}$ and $\mathrm{N}_{2}$ in Athabasca Bitumen. Canadian Journal of Chemical Engineering, 80, 116-125.

Upreti, S.R., Lohi, A., Kapadia, R.; El-Haj, R., 2007. Vapor Extraction of Heavy Oil and Bitumen: A Review. Energy \& Fuels, 21, 1562-1574.

Wen, Y., Kantzas, A. and Wang, G.J., 2005. Estimation of Diffusion Coefficients in Bitumen Solvent Mixtures Using X-Ray CAT Scanning and Low Field NMR. Proceedings: Canadian International Petroleum Conference, 8-10 June 2004, Calgary, Alberta, Canada, Paper 2004-270. Wikimedia, 2006:

http://upload.wikimedia.org/wikipedia/commons/thumb/7/7a/Athabasca_Oil_Sands_map.png/25 Opx-Athabasca_Oil_Sands_map.png (accessed 20 June, 2010).

Yang, C. and Gu, Y., 2006. Diffusion Coefficients and Oil Swelling Factors of Carbon Dioxide, Methane, Ethane, Propane, and their Mixtures in Heavy Oil. Fluid Phase Equilibria, 243, 64-73.

Yazdani, A., and Maini, B., 2009. The Effective Diffusion/Dispersion Coefficient in Vapor Extraction of Heavy Oil. Journal of Petroleum Science and Technology, 27, 817-835.

Yazdani, A.J. and Maini, B.B. 2005. Effect of Drainage Height and Grain Size on Production Rates in the Vapex Process: Experimental Study. SPE Reservoir Evaluation and Engineering, June 2005, 205-213, Paper SPE-89409-PA. 


\section{Appendix A}

Sample of porous medium permeability calculation

The permeability (K) was calculated by applying the following equation (Dullien, 1992):

$K=\frac{P_{2} \times V_{2} \times \mu_{\mathrm{air}} \times L}{P_{\mathrm{m}} \times \Delta P}$

where $P_{1}$ and $P_{2}$ are the pressures at the inlet and outlet of the cylinder, $V_{2}$ is the velocity at the outlet, $P_{\mathrm{m}}$ is the mean pressure, $\Delta P$ is the pressure difference, $\mu_{\mathrm{air}}$ is the air viscosity at room temperature, and substituting for the parameters from Table A.

Table A Data for calculations

\begin{tabular}{l|c}
\hline Parameter & Value \\
\hline $\mathrm{P}_{1}($ atm) & 1.153 \\
\hline $\mathrm{P}_{2}($ atm) & 1.142 \\
\hline $\mathrm{Q}_{2}\left(\mathrm{~cm}^{3} / \mathrm{s}\right)$ & 61.96 \\
\hline $\mathrm{L}(\mathrm{cm})$ & 26 \\
\hline $\mathrm{D}(\mathrm{cm})$ & 4 \\
\hline $\mathrm{V}_{2}(\mathrm{~cm} / \mathrm{s})$ & 4.93 \\
\hline$\Delta \mathrm{P}(\mathrm{atm})$ & 0.0106 \\
\hline $\mathrm{P}_{\mathrm{m}}(\mathrm{atm})$ & 1.147 \\
\hline$\mu_{\text {air }}(c P)$ & 0.0184 \\
\hline $\mathrm{K}($ Darcy) & 222.438 \\
\hline
\end{tabular}

$$
\begin{aligned}
K & =\frac{1.142 \times 4.93 \times 0.0184 \times 26}{1.147 \times 0.0106} \\
& =222.438 \text { Darcy }
\end{aligned}
$$




\section{Appendix B}

Sample of Live Oil Viscosity Calculations

The live oil viscosity was calculated by rearranging Equation (3.2) and substituting for the parameters from Table B.

Table B Data for live oil viscosity calculations

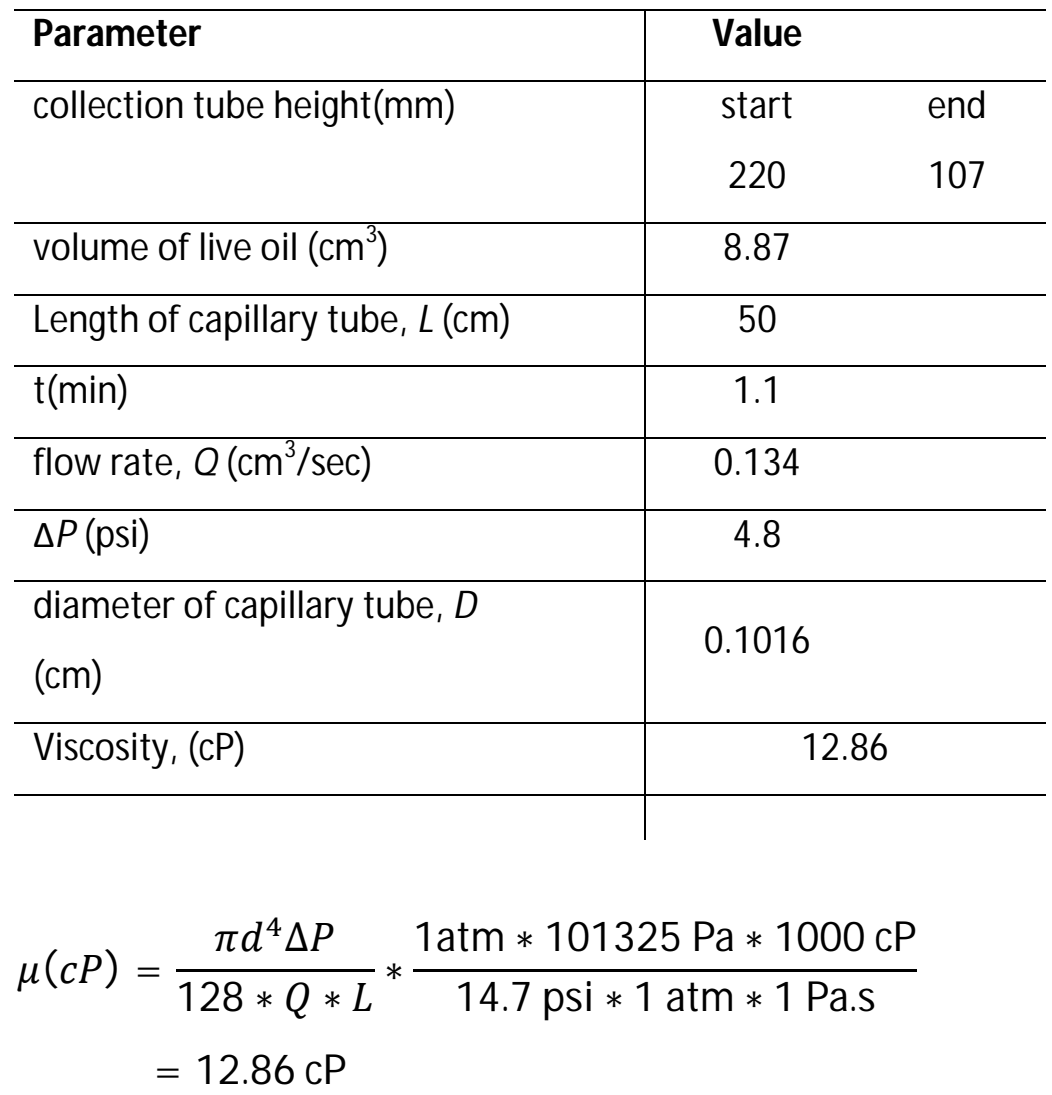




\section{Appendix C}

Sample of live oil density $\left(\rho_{\text {live }}\right)$ and amount of propane dissolved in heavy oil $(\omega)$ calculations The live oil viscosity was calculated using Equations (3.3) and (3.4) and substituting for the parameters from Table C.

Table C Data for calculations

\begin{tabular}{l|c|c}
\hline \multicolumn{1}{c|}{ Parameter } & \multicolumn{2}{c}{ Value } \\
\hline height of live oil in collection tube & Initial(mm) & Final(mm) \\
\hline volume of live oil $\left(\mathrm{cm}^{3}\right)$ & 145 & 50 \\
\hline height of water column(in) & 7.457 & \\
\hline volume of propane $\left(\mathrm{cm}^{3}\right)$ & 0 & 14 \\
\hline mass of propane $(\mathrm{g})$ & 1004.92 & \\
\hline mass of dead oil $(\mathrm{g})$ & 1.841 & \\
\hline mass fraction of propane & 4.23 & \\
\hline density of live oil $\left(\mathrm{g} / \mathrm{cm}^{3}\right)$ & 0.3032 & \\
\hline
\end{tabular}

$\omega=\frac{\text { mass of librated propane }}{\text { mass of dead oil + mass of librated propane }}$

$$
\begin{aligned}
& =\frac{\left[\pi * 3^{2} *(14 * 2.54)\right] * 0.001832}{0.001832 * 1004.92+4.23} \\
& =0.3032
\end{aligned}
$$

$$
\begin{aligned}
\rho_{\text {live }} & =\frac{\text { mass of librated propane }+ \text { mass of dead oil }}{\text { volume of live oil }} \\
& =\frac{1.841+4.23}{\pi * 0.5^{2} *(145-50) * 0.1} \\
& =0.814 \mathrm{~g} / \mathrm{cm}^{3}
\end{aligned}
$$




\section{Appendix D}

Recorded mass of the physical model versus time in an experiment

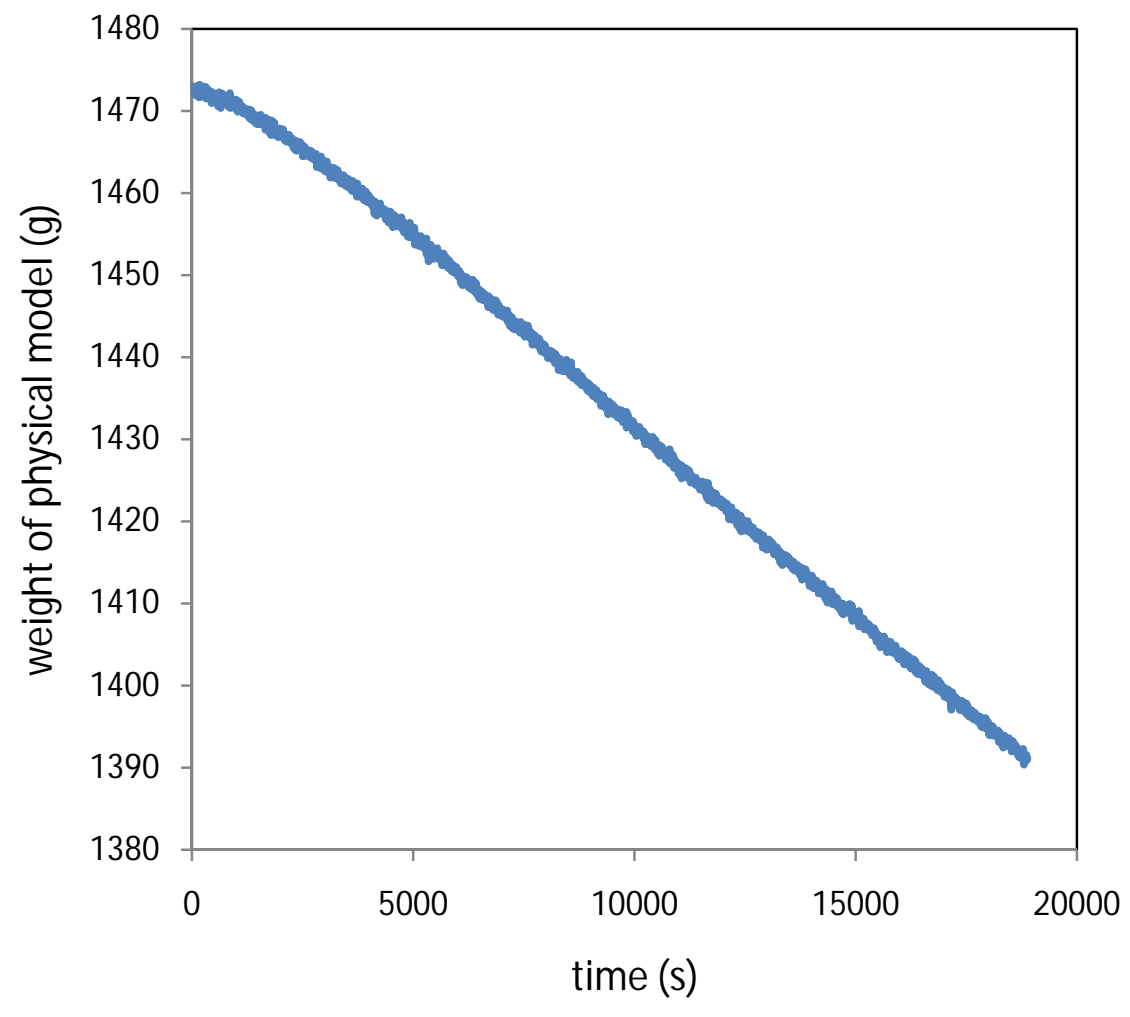

Figure D.1 Recorded mass of the physical model with time 


\section{Appendix E}

Relative deviations of the pressure vessel temperature and the water bath temperature.

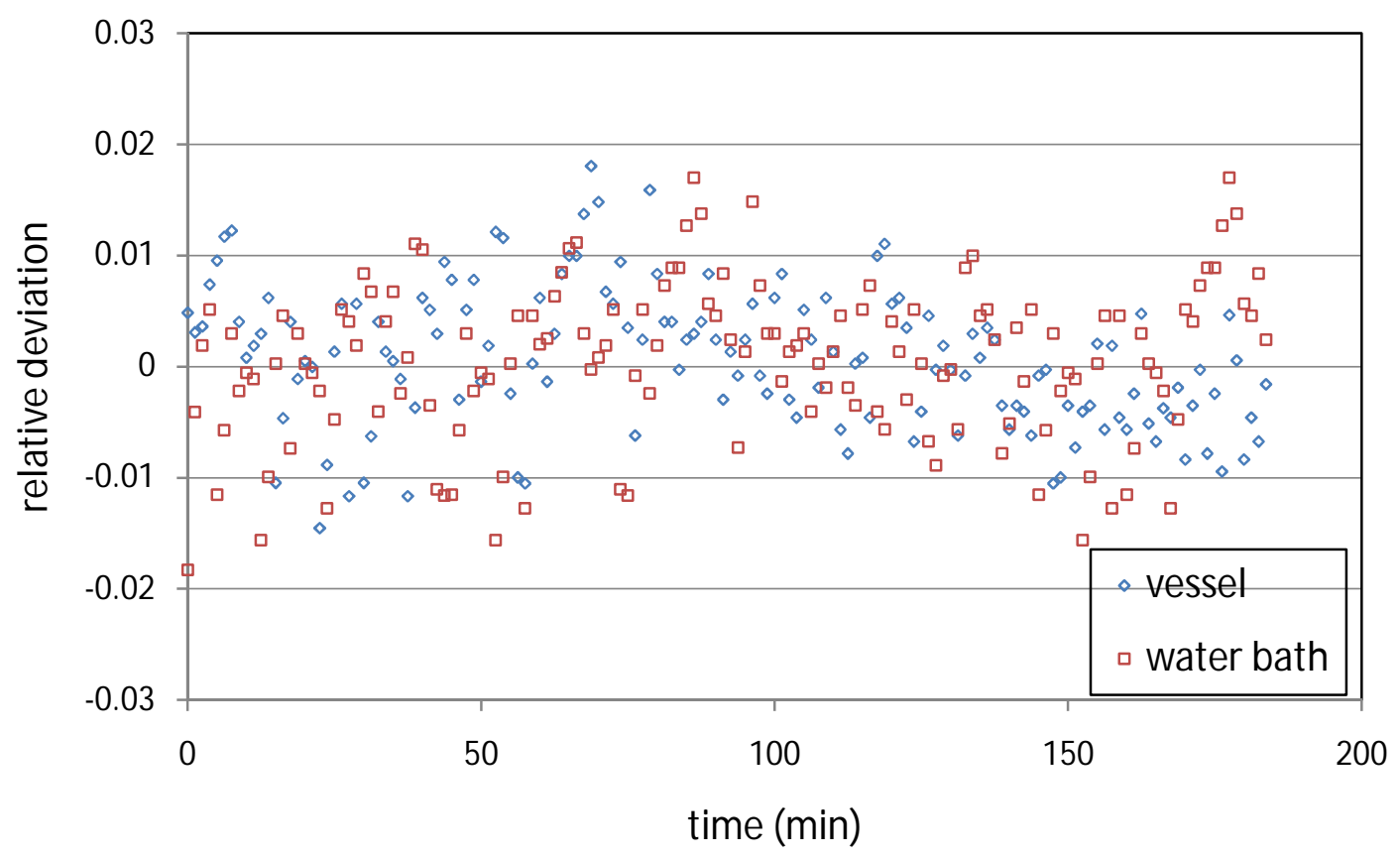

Figure E.1 Relative deviation versus time 


\section{Appendix F}

The cumulative oil production curves for various physical models

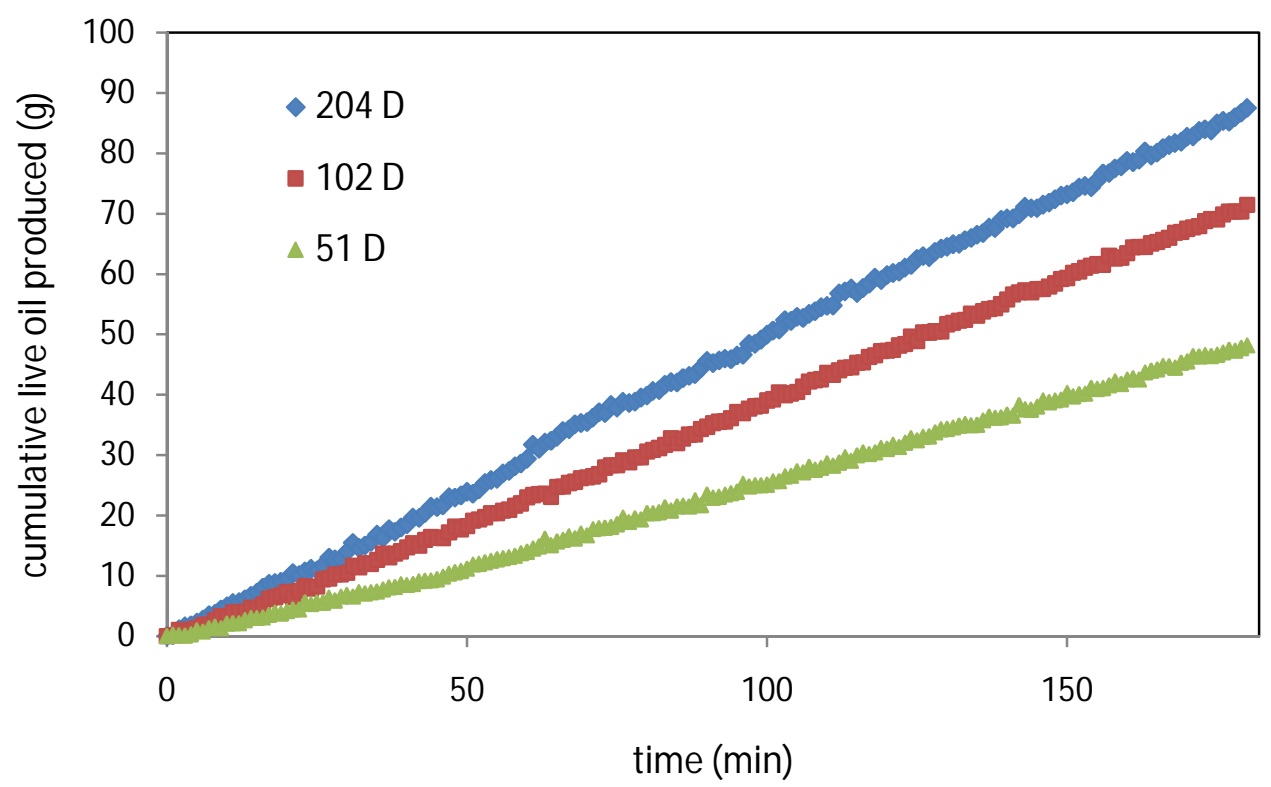

Figure F.1 Cumulative live oil production for homogeneous packing in $25 \mathrm{~cm}$ model versus time

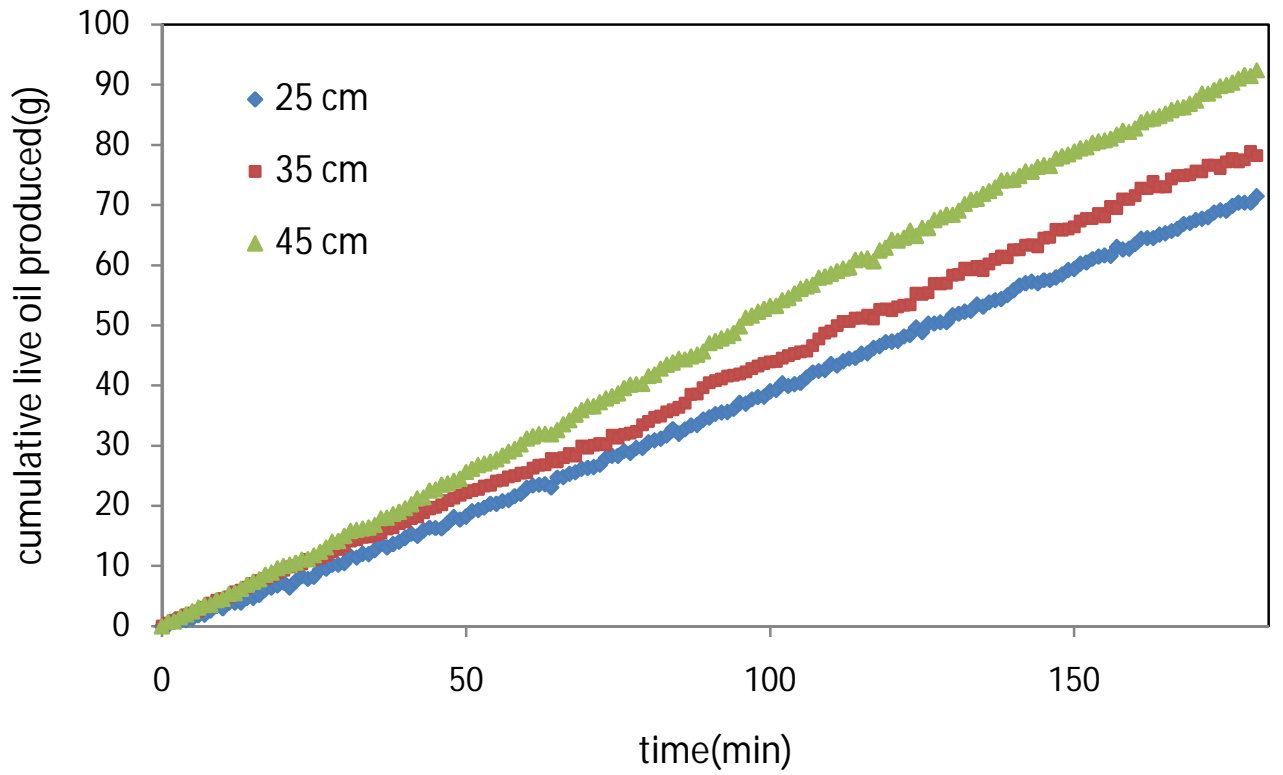

Figure F.2 Cumulative live oil production for homogeneous packing of $204 \mathrm{D}$ model versus time 


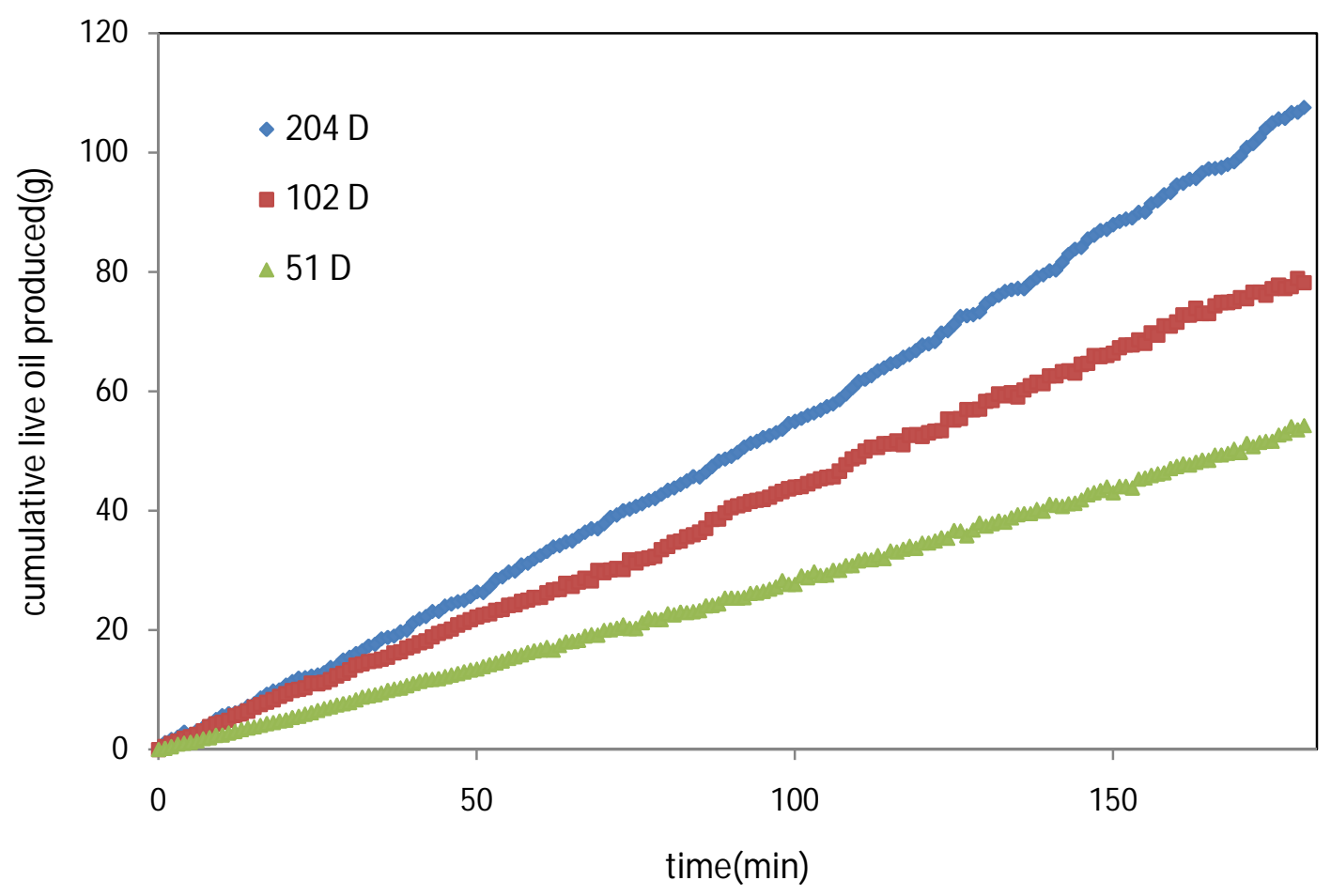

Figure F.3 Cumulative live oil production for homogeneous packing of $\mathbf{3 5} \mathrm{cm}$ model versus time

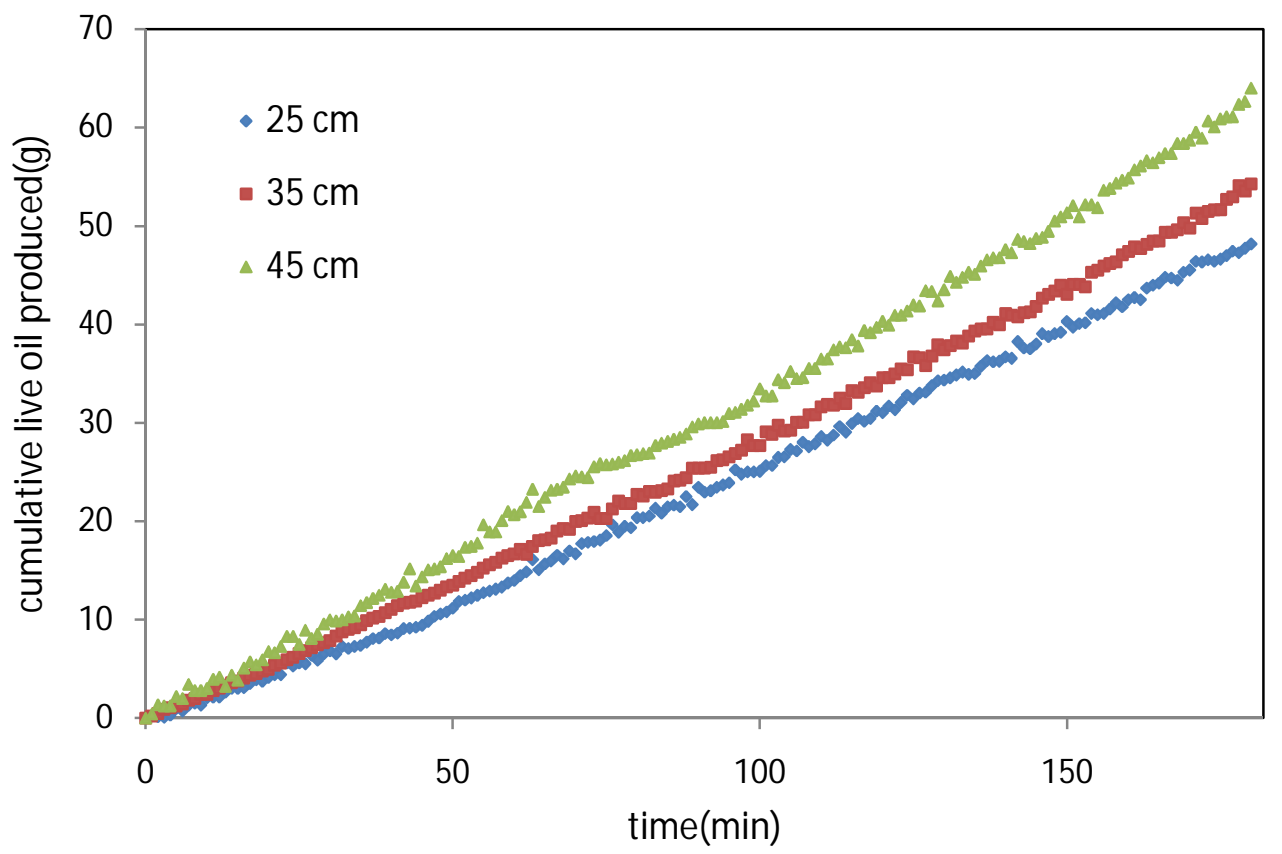


Figure F.4 Cumulative live oil production for homogeneous packing of $102 \mathrm{D}$ model versus time

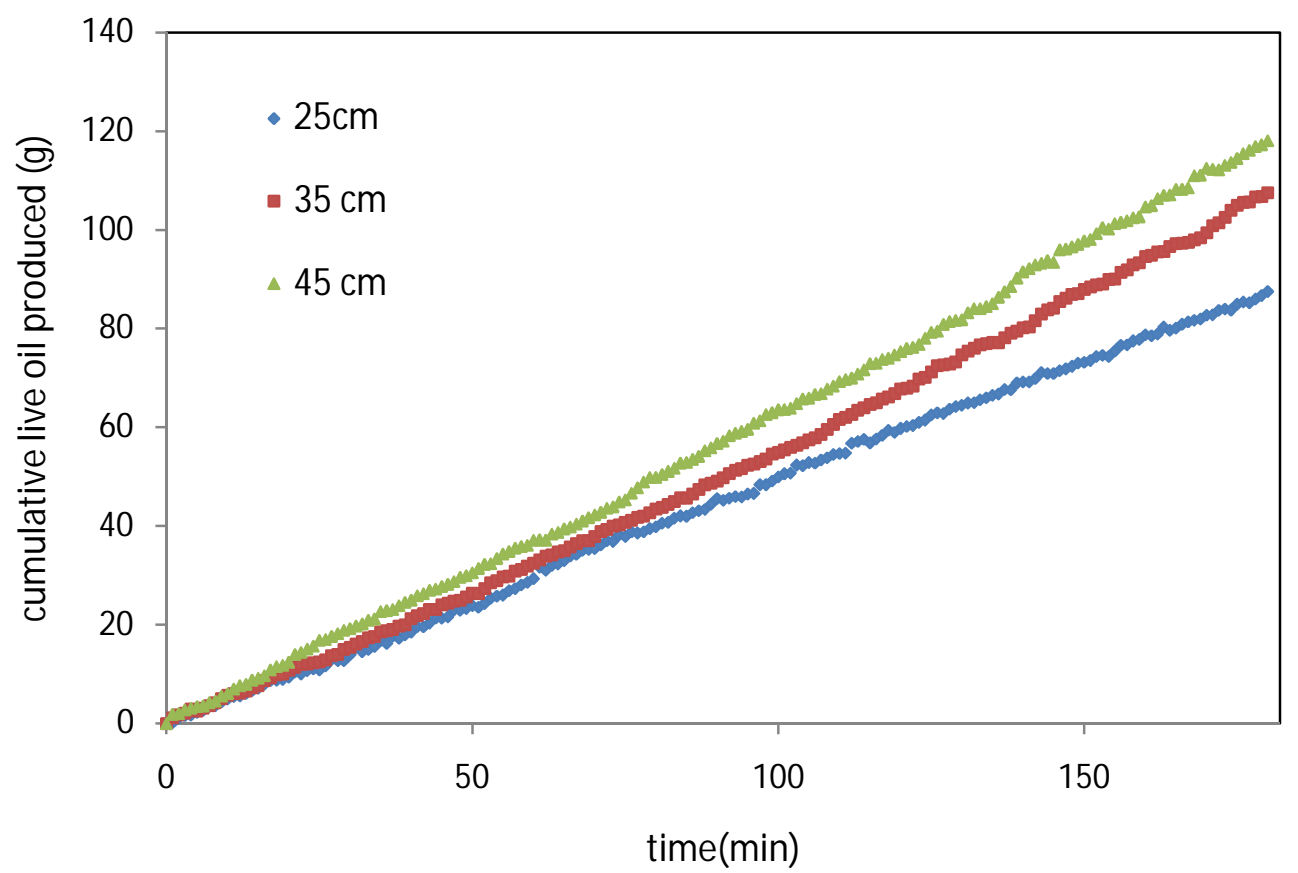

Figure F.5 Cumulative live oil production for homogeneous packing of $51 \mathrm{D}$ model versus time 


\section{Appendix G}

A comparison between the simulation results for both 60 minutes and 120 minutes simulation time for a physical model of $25 \mathrm{~cm}$ drainage height and a permeability of $204 \mathrm{D}$. The average absolute error in dispersion values is $0.101 \times 10^{-5} \mathrm{~m}^{2} / \mathrm{s}$.

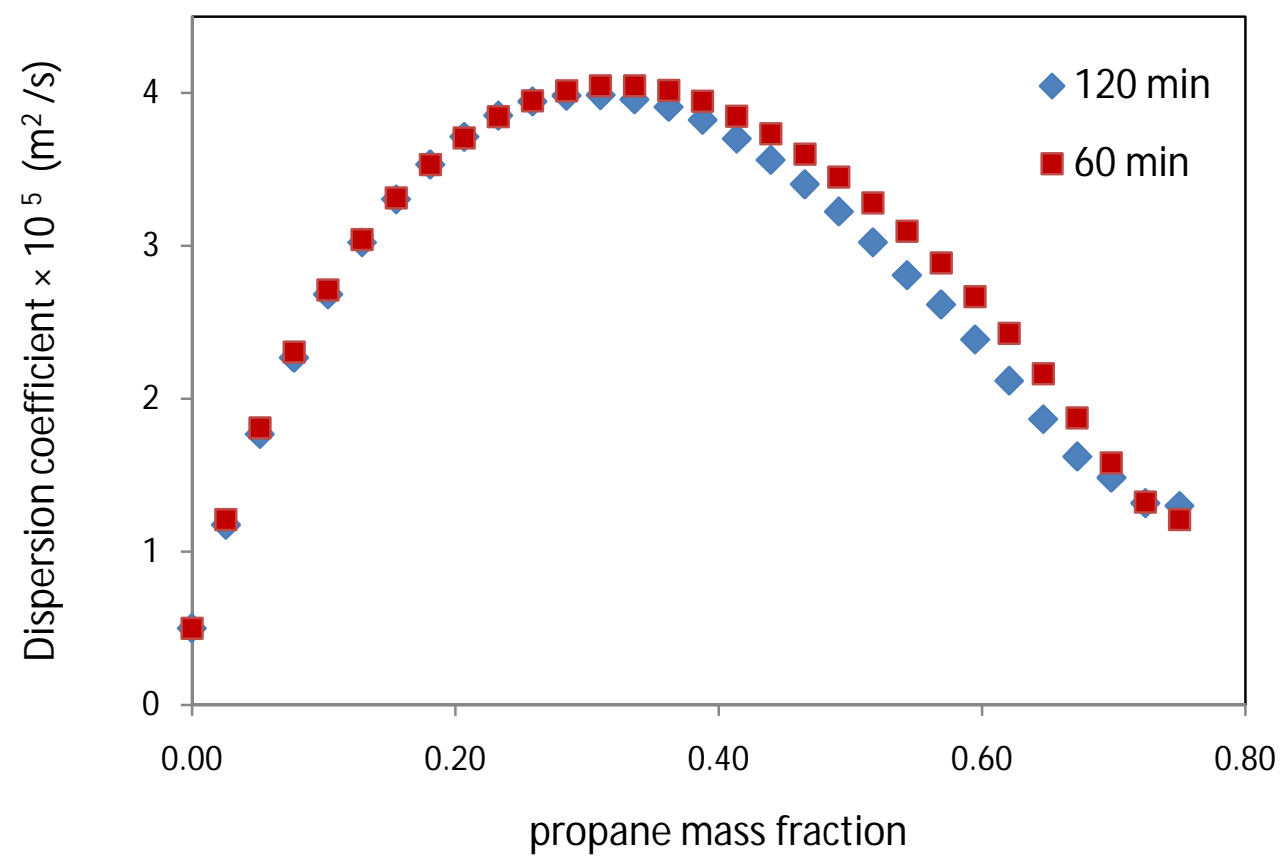

Figure G.1 Propane dispersion coefficient versus propane mass fraction $(204 \mathrm{D}, 25 \mathrm{~cm})$

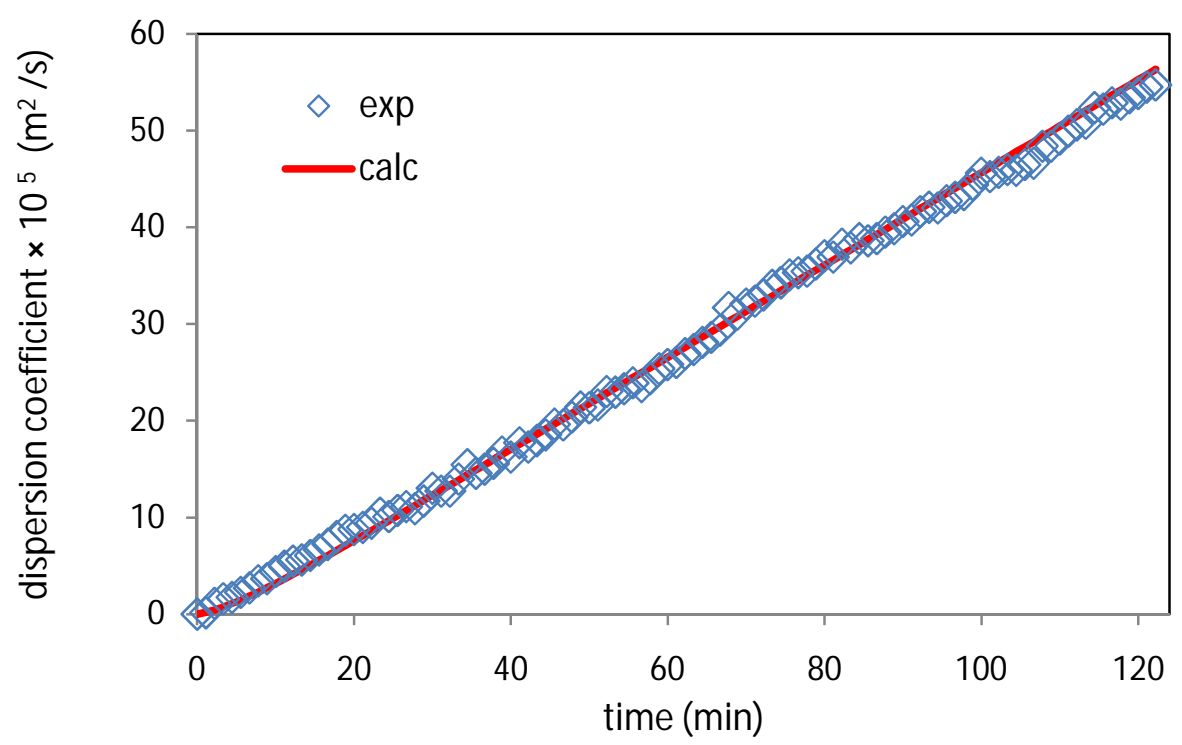

Figure G.2 Experimental and predicted live oil produced (204 D, $25 \mathrm{~cm}, 120 \mathrm{~min})$ 


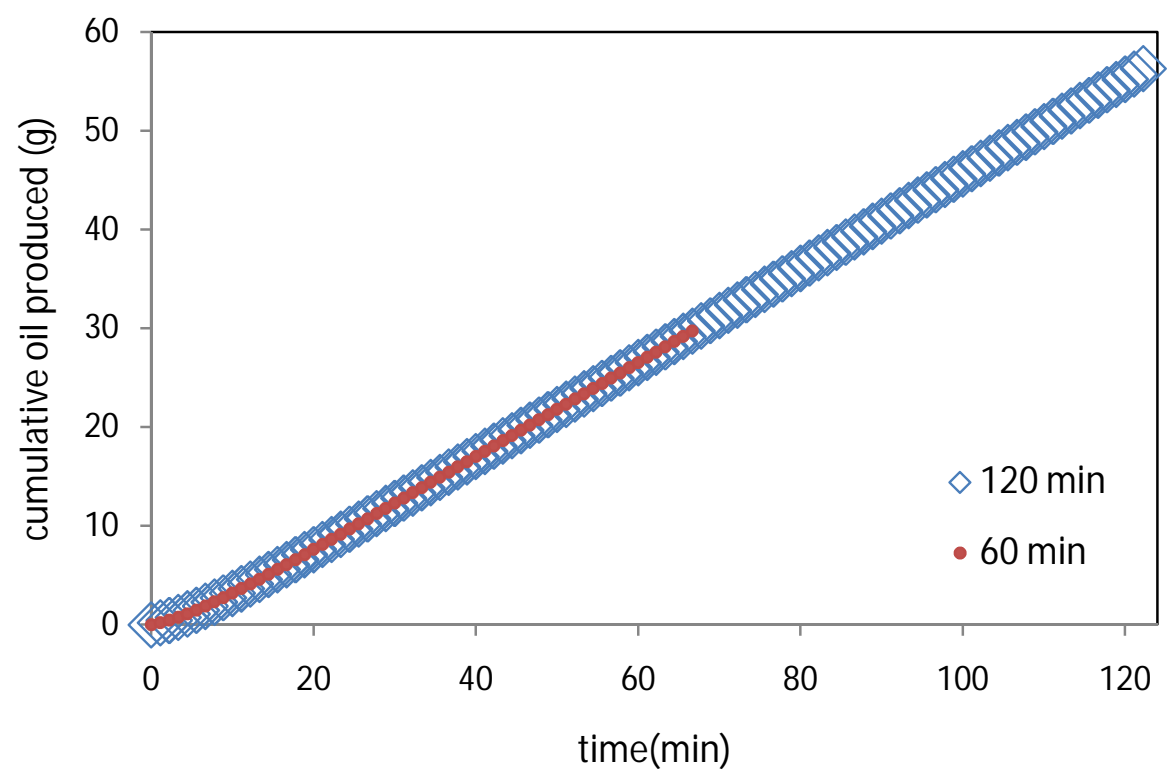

Figure G.3 Comparison for cumulative predicted live oil produced versus time (204 D, 25 cm)

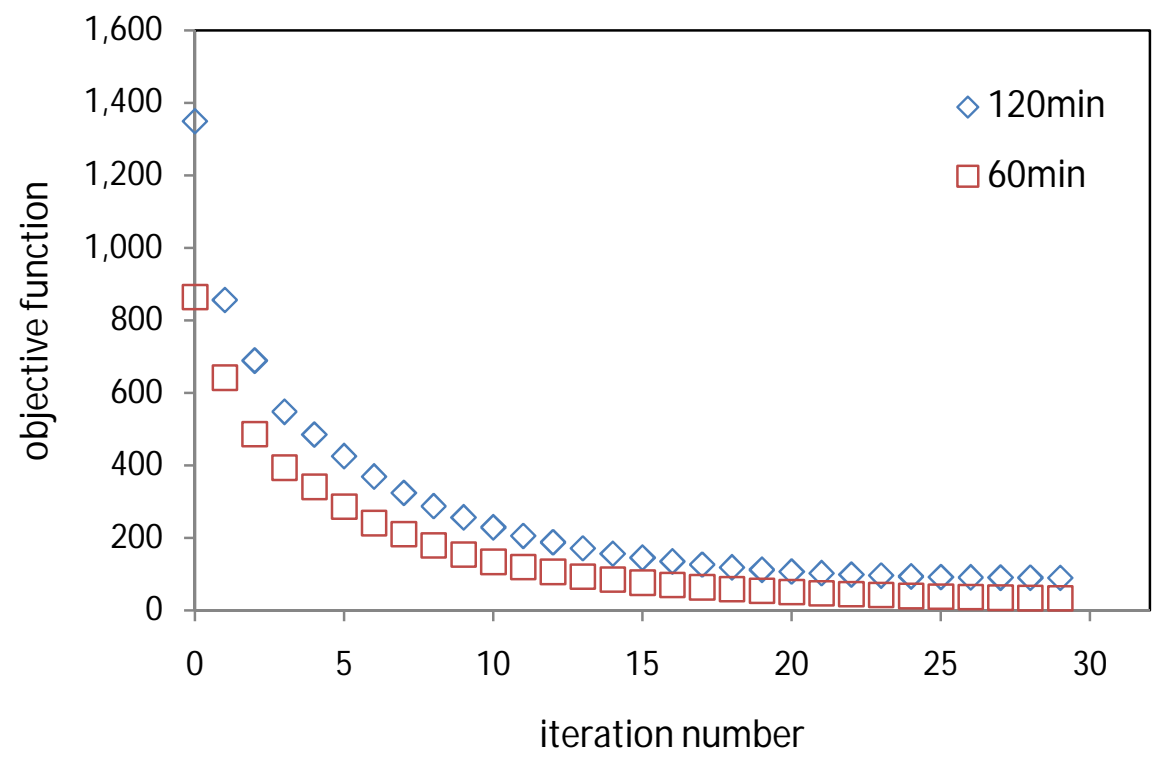

Figure G.4 Objective function versus iteration number $(204 \mathrm{D}, 25 \mathrm{~cm})$ 
Similarly, a comparison between the simulation results for both 60 minutes and 120 minutes simulation time for a physical model of $35 \mathrm{~cm}$ drainage height and a permeability of $204 \mathrm{D}$. The average absolute error in dispersion values is $0.133 \times 10^{-5} \mathrm{~m}^{2} / \mathrm{s}$.

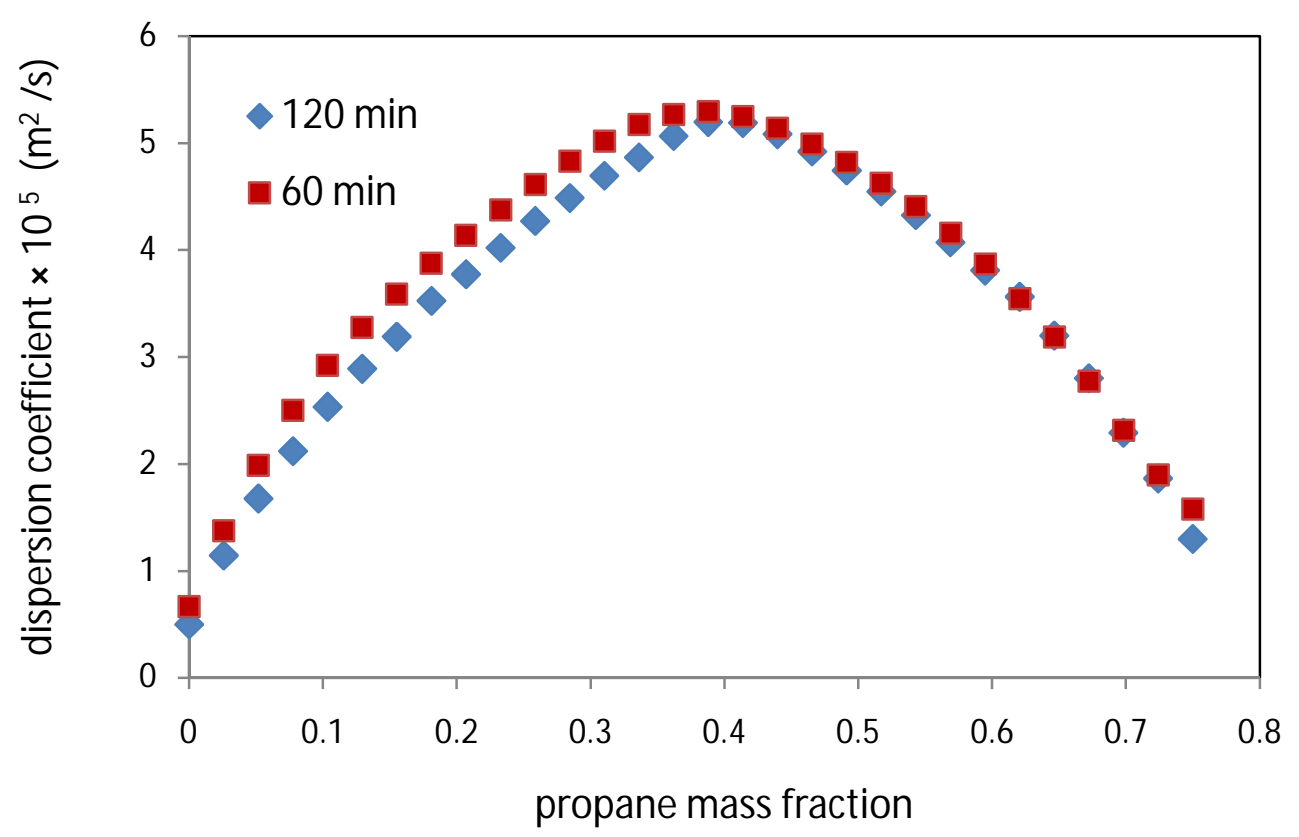

Figure G.5 Propane dispersion coefficient versus propane mass fraction $(204,35 \mathrm{~cm})$ 


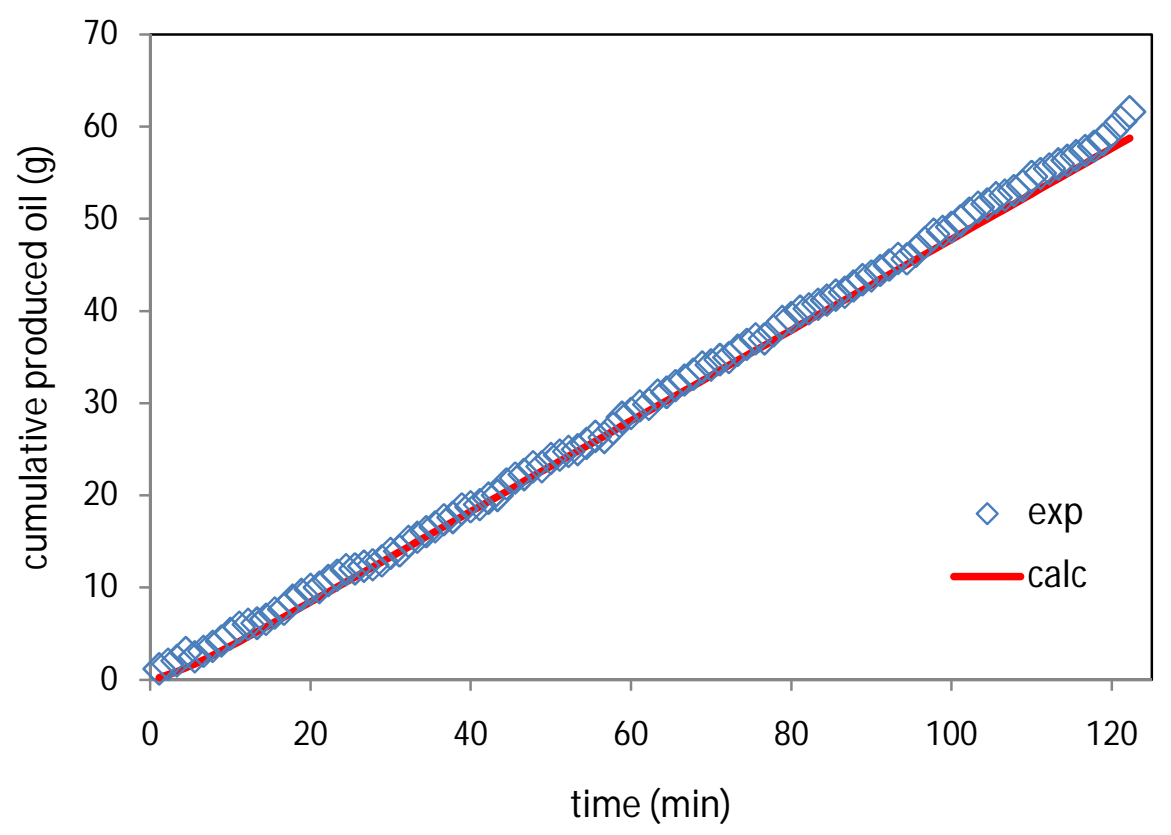

Figure G.6 Experimental and predicted live oil produced (204 D, 35 cm, $120 \mathrm{~min})$

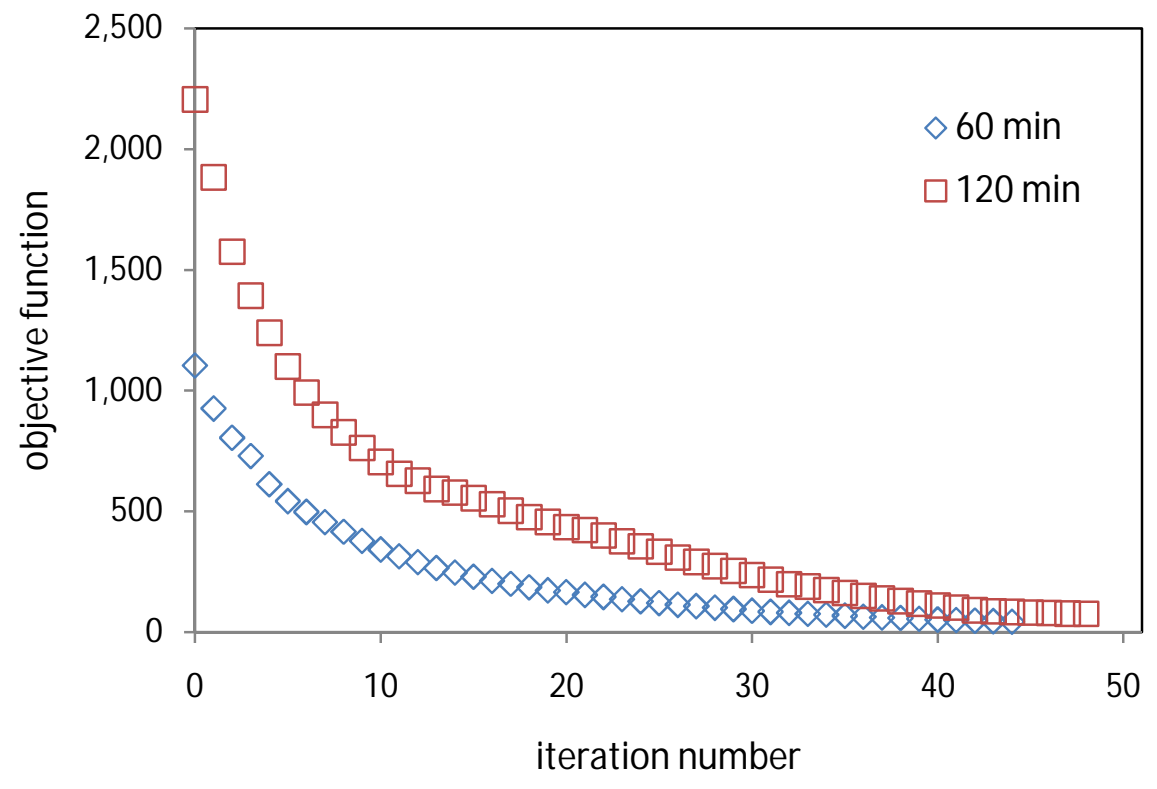

Figure G.7 Objective function versus iteration number $(204 \mathrm{D}, 35 \mathrm{~cm})$ 


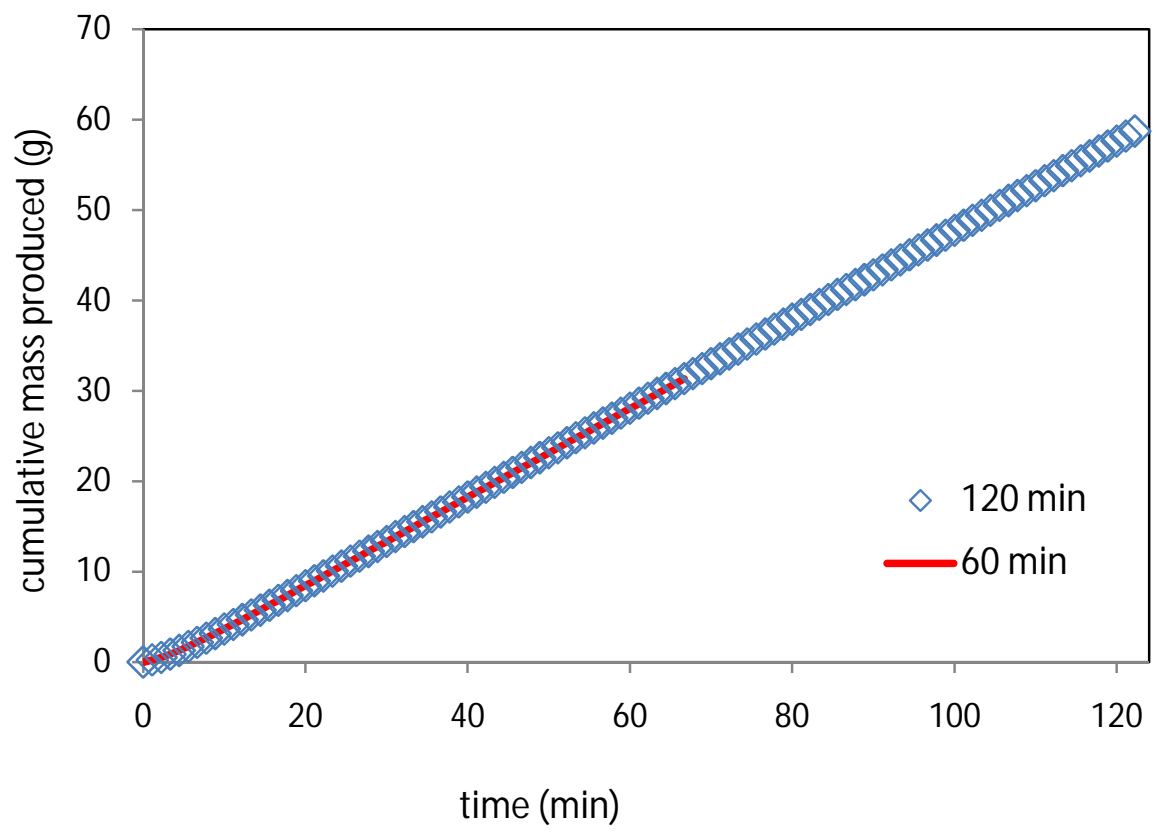

Figure G.8 Comparison for cumulative predicted live oil produced versus time (204 D, 35 cm) 


\section{Appendix H}

Justification for the extrapolation of the viscosity model

Figure H.1, shows the experimental live oil viscosity as a function of propane mass fraction in the produced live oil (triangle symbol). Moreover, the same figure shows the extrapolated data generated based on the viscosity model (circle symbol) (case I). Now, the experimental data is extrapolated to the point, where the viscosity of the mixture is equal to the viscosity of pure propane $(0.096 \mathrm{cP})$ at the operating conditions (case II). The average relative error between the viscosities calculated for both cases is $26 \%$. The effect of this error on the dispersion values is found to be insignificant by conducting a sensitivity study. The average change in the dispersion values is found to be $0.3 \times 10^{-5} \mathrm{~m}^{2} / \mathrm{s}$.

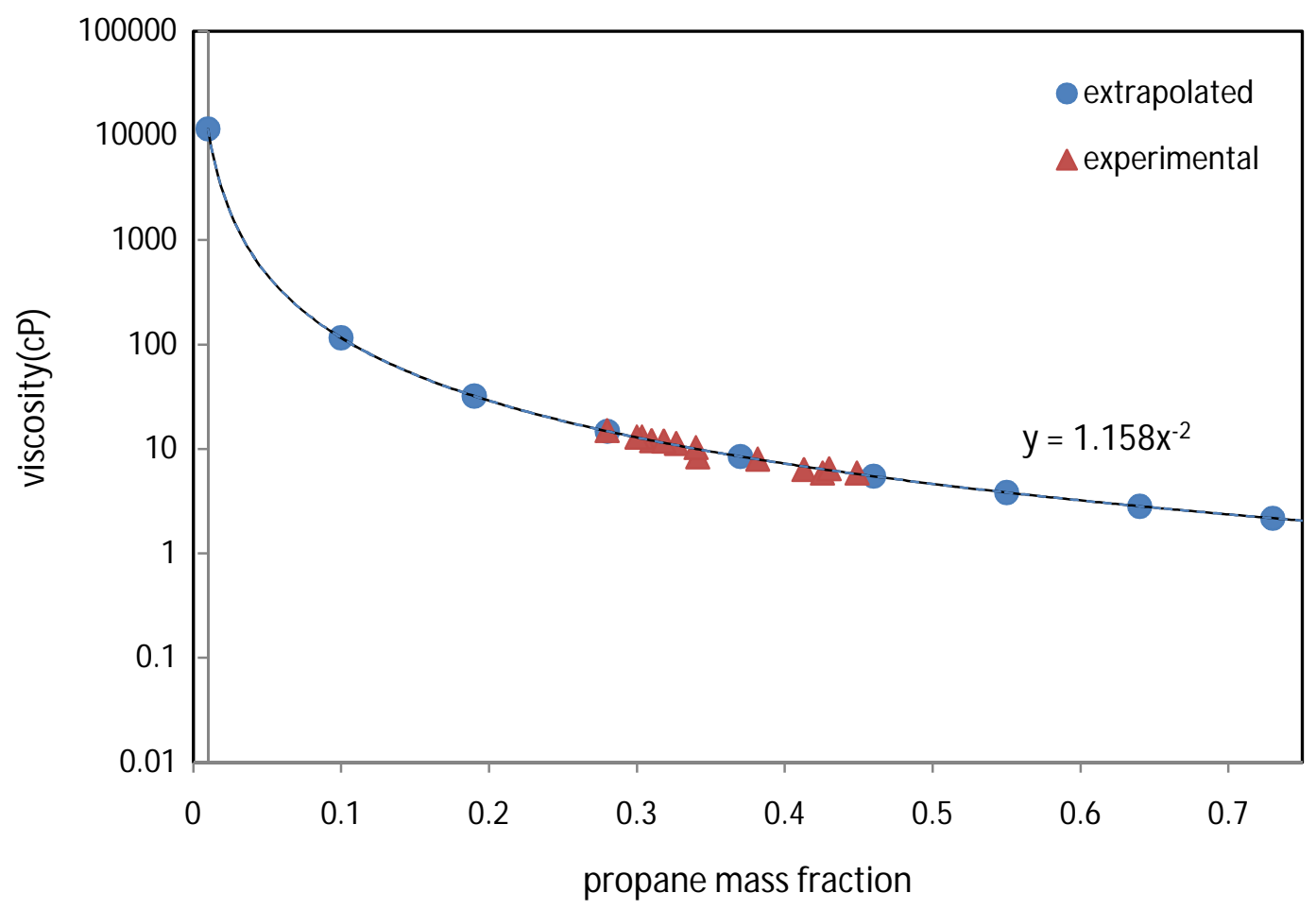

Figure H.1 Live oil viscosity versus propane mass fraction 


\section{Appendix I}

The velocity profile at the bottom of the physical model $(25 \mathrm{~cm}$ drainage height, $204 \mathrm{D}$ permeability).



Figure I.1 Velocity at the bottom of the physical model versus radius 